\title{
Tópicos de álgebras alternativas
}

\author{
Marcos Munhoz
}

\author{
Dissertação apresentada \\ ao \\ Instituto de Matemática e Estatística \\ da \\ Universidade de São Paulo \\ para \\ obtenção do grau de Mestre \\ em
}

Ciências

Área de concentração: Matemática

Orientador: Prof. Dr. Henrique Guzzo Junior

São Paulo, fevereiro de 2007 


\section{Tópicos de álgebras alternativas}

Este exemplar corresponde à redação final da dissertação devidamente corrigida e defendida por Marcos Munhoz e aprovada pela Comissão Julgadora.

São Paulo, 23 de fevereiro de 2007.

Banca Examinadora:

Prof. Dr. Henrique Guzzo Junior (orientador) - IME/USP

Prof. Dr. Juan Carlos Gutiérrez Fernández - IME/USP

Prof. Dr. Plamen Emilov Kochloukov - IMECC/UNICAMP 


\section{Resumo}

Neste trabalho são apresentados alguns aspectos das álgebras alternativas, em especial o bar radical de uma álgebra bárica alternativa e as identidades de graus 4 e 5 nas álgebras de Cayley-Dickson. Estudamos alguns resultados importantes sobre as álgebras báricas alternativas, em particular aqueles envolvendo o radical, o bar ideal e o bar radical, fazendo uso da Decomposição de Peirce. No estudo das identidades nas álgebras de Cayley-Dickson, foram utilizadas diversas técnicas e propriedades das álgebras alternativas, onde mostramos que não existem identidades de grau 4, além das triviais e que as identidades de grau 5 são conseqüências de outras duas identidades conhecidas.

\footnotetext{
Abstract

In this work we present some aspects of alternative algebras, in special the bar radical of an alternative baric algebra and identities of degree 4 and 5 of Cayley-Dickson algebras. We studied some important results about alternative baric algebras, particularly those related with the radical, the bar-ideal and the bar radical, with the use of the Peirce decomposition. In the study of the identities in Cayley-Dickson algebras we made wide use of several techniques and properties of alternative algebras, in order to show that the only identities of degree four are the trivial ones, and the identities of degree five are consequences of other two known identities.
} 


\section{Sumário}

Introdução $\quad 6$

1 Álgebras Alternativas $\quad 7$

1.1 Conceitos Básicos . . . . . . . . . . . . . . . . . 7

1.2 Álgebras Alternativas . . . . . . . . . . . . . . . . . . . . 10

1.3 Decomposição de Peirce . . . . . . . . . . . . . . . . . . . . 21

1.4 Álgebras Báricas . . . . . . . . . . . . . . . . . . . . . . . 31

2 Radical e Semisimplicidade 36

2.1 O Radical . . . . . . . . . . . . . . . . . . . . 36

2.2 Decomposição de Peirce em álgebras báricas . . . . . . . . . . . . . . 50

2.3 O radical e o bar radical . . . . . . . . . . . . . . . 56

3 Álgebras de Composição $\quad 70$

3.1 A descoberta dos quatérnios . . . . . . . . . . . . . . . . 71

3.2 As Álgebras de Composição . . . . . . . . . . . . . . . . . . . 73

3.3 Processo de Duplicação de Cayley-Dickson . . . . . . . . . . . . . . . 79

3.4 Exemplos de Álgebras de Composição . . . . . . . . . . . . . . . . . . 84

3.5 Hurwitz e a Soma de Quadrados . . . . . . . . . . . . . . . . . . 88

4 Identidades em Álgebras de Cayley $\quad 92$

4.1 Introdução . . . . . . . . . . . . . . . . . . . . . . . . . . . . . 92 92

4.2 Álgebras Livres . . . . . . . . . . . . . . . . . . . . . . . . . . 93

4.3 Identidades Polinomiais . . . . . . . . . . . . . . . . . . . 96

4.4 A Álgebra Exterior - Principais Resultados . . . . . . . . . . . . . . . 101

4.5 Identidades nos Octônios - Grau 4 . . . . . . . . . . . . . . . . 109 
4.6 Identidades nos Octônios - Grau 5 . . . . . . . . . . . . . . . . . 115

4.7 Resultados Recentes . . . . . . . . . . . . . . . . . . . . 146

Bibliografia 


\section{Introdução}

O presente trabalho é um estudo das álgebras alternativas tanto em seus aspectos gerais como em manifestações particulares, que são as álgebras báricas e as álgebras de Cayley. Nas álgebras báricas indicamos importantes resultados relacionados ao radical e ao bar-radical. Nas álgebras de Cayley fazemos uma exposição das identidades, seja na sua determinação como na verificação de propriedades usando métodos matriciais.

O Capítulo 1 trata dos conceitos básicos das álgebras alternativas, estuda a sua decomposição de Peirce tanto em relação a um idempotente quanto em relação a um conjunto de idempotentes primitivos dois a dois ortogonais. São apresentadas também as idéias preliminares referentes às álgebras báricas, em especial, as álgebras báricas alternativas.

O Capítulo 2 estabelece os conceitos de radical e semisimplicidade, demonstrando os principais teoremas relacionados. São estudados o $\operatorname{bar}(A)$, o bar-radical e o radical nil de $A$, além da semisimplicidade no sentido bárico e no sentido usual.

O Capítulo 3 é dedicado a um estudo teórico das álgebras de composição, descrevendo o processo de duplicação de Cayley-Dickson que permite obter todas as álgebras de composição. Demonstramos o Teorema de Hurwitz sobre as álgebras de composição.

O Capítulo 4 se refere às identidades nas álgebras de Cayley, em particular, as de grau 4, com resultados fundamentados nos teoremas de Amitsur-Levitzki, e as identidades de grau 5, onde mostramos que são conseqüências de outras identidades resultantes do conceito de derivações em álgebras de Jordan. Concluímos o trabalho mencionando alguns resultados recentes sobre as identidades de grau 6 nas álgebras de Cayley e de grau 7 nas álgebras alternativas, em que alguns autores utilizaram métodos computacionais na sua pesquisa. 


\section{Capítulo 1}

\section{Álgebras Alternativas}

As álgebras alternativas são um importante exemplo de álgebras em que a propriedade associativa não é assumida em sua definição, mas que pode valer em casos particulares. Uma ampla gama de aplicações são conhecidas e seu estudo conduz a resultados gerais que permitem estabelecer de forma inequívoca uma série de propriedades destas álgebras. Daremos neste capítulo as definições básicas e os principais resultados, para no capítulo seguinte aprofundarmos um pouco mais este estudo.

\subsection{Conceitos Básicos}

Vamos trabalhar com álgebras sobre um corpo $F$. Uma álgebra $A$ sobre um corpo $F$ é um espaço vetorial onde está definido um produto de elementos de $A$, satisfazendo, para todos $x, y, z$ em $A$ e $\alpha$ em $F$ :

$$
\begin{gathered}
(x+y) z=x z+y z \\
x(y+z)=x y+x z \\
\alpha(x y)=(\alpha x) y=x(\alpha y)
\end{gathered}
$$

Definição 1.1.1. Seja A uma álgebra.

(i) $S e(x y) z=x(y z) \quad \forall x, y, z \in A$, dizemos que A é uma álgebra associativa.

(ii) Sejam $x, y, z \in A$. Definimos o associador de A por: $(x, y, z)=(x y) z-x(y z)$.

(iii) O núcleo de uma álgebra $A$, denotado por $Z$ é o conjunto dos elementos z em $A$ que se associam com todos os elementos de $A$.

$z \in Z \Leftrightarrow \forall x, y \in A,(x, y, z)=(x, z, y)=(z, x, y)=0$. 
(iv) O centro de uma álgebra $A$, denotado por $\mathcal{C}$ é o conjunto dos elementos $c$ em A que comutam e se associam com todos os elementos de $A$.

$\mathcal{C}=\{c \in Z \mid x c=c x, \forall x, y \in A\}$

Se $A$ é associativa, então $A=Z$. No caso geral: $\mathcal{C} \subseteq Z \subseteq A$.

Definição 1.1.2. Sejam $A$ uma álgebra e $X, I, B, C$ subconjuntos de $A$.

(i) X é chamado uma subálgebra de $A$ se $X$ é um subespaço vetorial de $A$ e $X$ é um subanel de $A$. Notação: $X \leq A$, ou $X<A$, se $X \neq A$.

(ii) I é chamado ideal de A se I é um ideal de $A$ considerando $A$ como anel $e$ $\forall \alpha \in F, \forall a \in A$, tem-se: $\alpha a \in I$.

(iii) Indicaremos por $\langle X\rangle$ o subespaço de A gerado por $X$.

(iv) De modo usual a subálgebra de A gerada por $X$ é a intersecção de todas as subálgebras de $A$ que contêm $X$. Denotamos por $A(X)$. Quando $X=$ $\left\{x_{1}, \ldots, x_{n}\right\}$ escrevemos $A\left(\left\{x_{1}, \ldots, x_{n}\right\}\right)=A\left(x_{1}, \ldots, x_{n}\right)$.

(v) Indicamos por BC o seguinte subconjunto:

$$
B C=\langle b c \mid b \in B, c \in C\rangle
$$

Para ilustrar melhor o conjunto $B C$ acima definido, note que:

$$
\begin{gathered}
u \in B C \Leftrightarrow \exists n \in \mathbb{N} \text { e } b_{1}, \ldots, b_{n} \in B, c_{1}, \ldots, c_{n} \in C, \alpha_{1}, \ldots, \alpha_{n} \in F \text { tais que } \\
u=\sum_{i=1}^{n} \alpha_{i} b_{i} c_{i}
\end{gathered}
$$

Definição 1.1.3. Sejam $A$ e $B$ álgebras sobre um corpo $F$. Uma aplicação $\phi: A \rightarrow B$ é chamada um homomorfismo entre $A$ e $B$ se valer, para todos $a, b \in A$ e $\alpha \in F$ :

(i) $\phi(a+b)=\phi(a)+\phi(b)$;

(ii) $\phi(\alpha a)=\alpha \phi(a)$;

(iii) $\phi(a b)=\phi(a) \phi(b)$

As potências em uma álgebra arbitrária devem ser definidas com especial atenção, uma vez que, se $A$ é uma álgebra associativa, a potência n-ésima de um elemento $x \in A$ é definida como: 


$$
x^{1}=x ; \quad x^{n}=\left(x^{n-1}\right) x, \quad \text { se } n>1
$$

Esta definição traz como conseqüência que:

$$
x^{n+m}=x^{n} x^{m}, \quad \forall x \in A, \quad \forall m, n \in \mathbb{N}
$$

o que é válido devido à lei associativa. Mas se $A$ é uma álgebra arbitrária, tal propriedade não vale sempre. A definição a seguir estabelece o conceito de potência no contexto mais geral.

Definição 1.1.4. Sejam $A$ uma álgebra, $B \subset A$ subálgebra e $x \in A$.

(i) A potência principal à esquerda e à direita de $\boldsymbol{x}$ é dada por:

${ }^{1} x=x \quad{ }^{n+1} x=x\left({ }^{n} x\right) \quad n \in \mathbb{N}$

$x^{1}=x \quad x^{n+1}=\left(x^{n}\right) x \quad n \in \mathbb{N}$

(ii) A potência principal à esquerda e à direita de $B$ é dada por:

${ }^{1} B=B \quad{ }^{n+1} B=B\left({ }^{n} B\right) \quad n \in \mathbb{N}$

$B^{1}=B \quad B^{n+1}=\left(B^{n}\right) B \quad n \in \mathbb{N}$

(iii) A potência principal de $B$ é dada por:

$B^{(1)}=B \quad B^{(n+1)}=\left\langle B B^{(n)}, B^{(n)} B\right\rangle \quad n \in \mathbb{N}$

(iv) A potência plena de $B$ é dada por:

$B^{[1]}=B \quad B^{[n+1]}=B^{[n]} B^{[n]} \quad n \in \mathbb{N}$

Definição 1.1.5. Sejam $A$ uma álgebra não-associativa, $B \subset A$ e $x \in A$.

(i) $x \in A$ é nilpotente à esquerda de indice $k$, se ${ }^{k} x=0$, mas ${ }^{k-1} x \neq 0$.

(ii) $B \subset A$, subálgebra é nil à esquerda se $x$ é nilpotente à esquerda, $\forall x \in B$.

(iii) $B \subset A$, subálgebra é nilpotente à esquerda de indice $k$, se ${ }^{k} B=0$, mas ${ }^{k-1} B \neq 0$.

(iv) $B$ é nilpotente de indice $k$, se $B^{(k)}=0$, mas $B^{(k-1)} \neq 0$. Se $k=2$, Bé chamada uma zero álgebra.

(iv) $B \subset A$ é solúvel de indice $k$ se $B^{[k]}=0$, mas $B^{[k-1]} \neq 0$. 
Definição 1.1.6. Seja A uma álgebra não-associativa e a $\in$ A. Definimos

(i) O operador multiplicativo à direita de A, determinado por a:

$$
\begin{aligned}
R_{a}: A & \rightarrow A \\
x & \mapsto x a
\end{aligned}
$$

(ii) O operador multiplicativo à esquerda de A, determinado por a:

$$
\begin{aligned}
L_{a}: A & \rightarrow A \\
x & \mapsto a x
\end{aligned}
$$

OBS.: $R_{a}$ e $L_{a}$ são operadores lineares.

\section{2 Álgebras Alternativas}

Uma álgebra $A$ sobre um corpo $F$ é chamada alternativa se, para todos $x, y \in A$, vale:

$$
\begin{aligned}
& x^{2} y=x(x y) \quad \text { (Lei alternativa à esquerda) } \\
& y x^{2}=(y x) x \quad \text { (Lei alternativa à direita) }
\end{aligned}
$$

Estas condições podem ser expressas em termos de associadores como segue:

$$
(x, x, y)=(y, x, x)=0
$$

Vamos mostrar algumas propriedades simples envolvendo as álgebras alternativas, as quais serão úteis em aplicações posteriores.

Teorema 1.2.1. Em uma álgebra alternativa $A$, tem-se, para todos $x, y, z$ em $A$ :

(i) $L_{x^{2}}=L_{x}^{2}, \quad R_{x^{2}}=R_{x}^{2}$;

(ii) $(x, y, z)=-(y, x, z)=(y, z, x)$;

(iii) $(x, y, x)=0 \quad$ (Lei Flexível);

(iv) $R_{x} L_{x}=L_{x} R_{x}$.

\section{Demonstração:}


(i) $y L_{x^{2}}=x^{2} y=x(x y)=(x y) L_{x}=\left(y L_{x}\right) L_{x}=y L_{x}^{2}$

$$
y R_{x^{2}}=y x^{2}=(y x) x=(y x) R_{x}=\left(y R_{x}\right) R_{x}=y R_{x}^{2}
$$

(ii) Para mostrar esta propriedade usamos a bilinearidade do associador:

$$
\begin{aligned}
0 & =(x+y, x+y, z)=(x, x, z)+(y, x, z)+(x, y, z)+(y, y, z) \\
& \Rightarrow 0+(y, x, z)+(x, y, z)+0=0
\end{aligned}
$$

Portanto: $(x, y, z)=-(y, x, z)$. Para a segunda igualdade temos:

$$
\begin{aligned}
0 & =(x, y+z, y+z)=(x, y, y)+(x, y, z)+(x, z, y)+(x, z, z) \\
& \Rightarrow(x, y, z)=-(x, z, y)
\end{aligned}
$$

Fazendo $x=y, y=z$ e $z=x$ obtemos: $(y, z, x)=-(y, x, z)$

(iii) $0=(x+y, y+x, x)=(x, y, x)+(x, x, x)+(y, y, x)+(y, x, x)$

$$
=(x, y, x)+0+0+0 \Rightarrow(x, y, x)=0
$$

(iv) É conseqüência direta da lei flexível.

Teorema 1.2.2 (Identidades de Moufang). Seja A uma álgebra alternativa. Para todos $a, x, y \in A$, são válidas as seguintes identidades:

(i) $(x a x) y=x(a(x y))$;

(ii) $(x y)(a x)=x(y a) x$;

(iii) $y(x a x)=((y x) a) x$.

\section{Demonstração:}

(i) $(x a x) y=x(a(x y))$

Lembrando que, pela lei flexível, podemos escrever sem ambigüidade $x a x$, uma vez que: $0=(x, a, x)=(x a) x-x(a x), \log$ o $(x a) x=x(a x)=x a x$. Calculemos: $(x a x) y-x(a(x y))$

$$
\begin{aligned}
(x a x) y-x(a(x y)) & =(x a, x, y)+(x, a, x y) \\
& =-(x, x a, y)-(x, x y, a)
\end{aligned}
$$




$$
\begin{aligned}
= & -\left(\left(x^{2} a\right) y-x((x a) y)\right)-\left(\left(x^{2} y\right) a-x((x y) a)\right) \\
= & -\left(x^{2} a\right) y-\left(x^{2} y\right) a+x((x a) y+(x y) a) \\
= & -\left(x^{2}, a, y\right)-x^{2}(a y)-\left(x^{2}, y, a\right)-x^{2}(y a) \\
& +x((x a) y+(x y) a) \\
= & x(-x(a y)-x(y a)+(x a) y+(x y) a) \\
= & x((x, a, y)+(x, y, a))=0
\end{aligned}
$$

(ii) $(x y)(a x)=x(y a) x$

$$
\begin{aligned}
(x y)(a x)-x(y a) x & =(x, y, a x)+x(y(a x))-x(y a) x \\
& =(x, y, a x)+x(y(a x)-(y a) x) \\
& =(x, y, a x)-x(y, a, x) \\
& =-(x, a x, y)-x(y, a, x) \\
& =x((a x) y)-(x a x) y-x(y, a, x) \\
& \stackrel{(i)}{=} x((a x) y-a(x y)-(y, a, x)) \\
& =x((a, x, y)-(y, a, x))=0
\end{aligned}
$$

(iii) $y(x a x)=((y x) a) x$

$$
\begin{aligned}
y((x a) x)-((y x) a) x & =[(y(x a)) x-(y, x a, x)]-[(y, x, a) x+(y(x a)) x] \\
& =(x a, y, x)-(x, a, y) x \\
& =((x a) y) x-(x a)(y x)-((x a) y) x+x(a y) x \\
& \stackrel{(i i)}{=}-x(a y) x+x(a y) x=0
\end{aligned}
$$

Corolário 1.2.3. Seja A uma álgebra alternativa. Então, para todos $a, x, y, z$ em $A$, tem-se: $(y, x a, z)+(y, z a, x)=-(y, x, a) z-(y, z, a) x$

Demonstração: A igualdade (iii) de Moufang equivale a: $(y, x a, x)=-(y, x, a) x$.

De fato:

$$
\begin{aligned}
(y, x a, x) & =(y(x a)) x-y(x a x)=(y(x a)) x-((y x) a) x=(y(x a)-(y x) a) x \\
& =-(y, x, a) x
\end{aligned}
$$


Linearizando a expressão $(y, x a, x)$, temos:

$$
\begin{aligned}
(y, x a+z a, x+z) & =(y,(x+z) a, x+z) \\
& =-(y, x+z, a)(x+z)=-(y, x+z, a) x-(y, x+z, a) z \\
& =-(y, x, a) x-(y, z, a) x-(y, x, a) z-(y, z, a) z
\end{aligned}
$$

Por outro lado, pela tri-linearidade do associador:

$$
\begin{aligned}
(y, x a+z a, x+z) & =(y, x a, x)+(y, x a, z)+(y, z a, x)+(y, z a, z) \\
& =-(y, x, a) x+(y, x a, z)+(y, z a, x)-(y, z, a) z
\end{aligned}
$$

Igualando (1) e (2), temos:

$$
\begin{aligned}
-(y, x, a) x & -(y, z, a) x-(y, x, a) z-(y, z, a) z \\
& =-(y, x, a) x+(y, x a, z)+(y, z a, x)-(y, z, a) z \\
& \Rightarrow(y, x a, z)+(y, z a, x)=-(y, x, a) z-(y, z, a) x
\end{aligned}
$$

Definição 1.2.1. Seja A uma álgebra e $x, y \in A$. Denotamos por $p=p(x, y)$ qualquer produto (não-associativo) de $t$ elementos $z_{1}, \ldots, z_{t}$, onde cada $z_{i}$ é igual a $x$ ou $y$. $O$ número $t$ de elementos no produto é chamado de grau de p, o qual denotaremos por $\partial p$. Esta definição se aplica igualmente para um produto de qualquer número de elementos, como $p=p(x, y, z), p=p(x, y, z, w)$, etc, onde $x, y, z, w \in A$.

\section{Exemplos}

(a) Seja $p=x^{2}(y x)$. Podemos escrever: $p=(x x)(y x)$. Portanto $\partial p=4$.

(b) $p=\left(x y^{2}\right)\left(z\left(x z^{3}\right)\right)$. Temos então: $\partial p=8$.

Teorema 1.2.4 (Artin). A subálgebra gerada por quaisquer dois elementos de uma álgebra alternativa $A$ é associativa.

Demonstração: Sejam $x, y \in A$ quaisquer. Seja $p=p(x, y)$ qualquer produto não-associativo $z_{1}, z_{2}, \ldots, z_{t}$, com alguma distribuição de parêntesis, onde $z_{i}=x$ ou $z_{i}=y$.

É suficiente provar que $(p, q, r)=0$ para todos os produtos não-associativos $p=$ $p(x, y), q=q(x, y)$ e $r=r(x, y)$. Vamos provar por indução em $n=\partial p+\partial q+\partial r$.

O resultado é imediato para $n<3$, pois, neste caso, pelo menos o grau de um dos produtos será zero. 
Assumimos como hipótese de indução que $(p, q, r)=0$ se $\partial p+\partial q+\partial r<n$. Então, como $\partial p<n$, no produto $p=p(x, y)$ vale a associativa. Desta forma os parêntesis não são necessários em $p(x, y)=z_{1} z_{2} \ldots z_{n}$ e podemos supor que $p$ começa com $z_{1}$.

Naturalmente este mesmo raciocínio vale para os outros produtos $q$ e $r$. Desta forma, dois dos produtos $p, q, r$ devem começar com a mesma letra. Vamos supor que $q$ e $r$ começam com $x$.

Temos 3 casos a considerar:

(1) $\partial q>1$ e $\partial r>1$

Temos que: $q=q^{\prime} x$ e $r=r^{\prime} x$. Daí

$\partial q=1+\partial q^{\prime} \Rightarrow \partial q^{\prime}=-1+\partial q$ e $\partial r=1+\partial r^{\prime} \Rightarrow \partial r^{\prime}=-1+\partial r$

Então: $\quad(p, q, r)=\left(p, x q^{\prime}, x r^{\prime}\right)=\left(x r^{\prime}, p, x q^{\prime}\right)=-\left(x r^{\prime}, x q^{\prime}, p\right)$

Na igualdade mostrada no Corolário 1.2.3, façamos as seguintes substituições:

$y=x r^{\prime}, \quad a=q^{\prime}, \quad z=p . \quad$ Resulta então:

$(y, x a, z)+(y, z a, x)=-(y, x, a) z-(y, z, a) x$

$\Rightarrow-(y, x a, z)=(y, z a, x)+(y, x, a) z+(y, z, a) x$

$\Rightarrow-\left(x r^{\prime}, x q^{\prime}, p\right)=\left(x r^{\prime}, p q^{\prime}, x\right)+\left(x r^{\prime}, x, q^{\prime}\right) p+\left(x r^{\prime}, p, q^{\prime}\right) x$

Como o segundo membro da expressão acima é igual a $(p, q, r)$, vamos determinar o grau dessa expressão, calculando o grau de cada parcela.

Ainda pelo Corolário 1.2.3, temos: $(y, x a, x)=-(y, x, a) x$. Daí, fazendo na primeira parcela as substituições indicadas:

$\left(x r^{\prime}, p q^{\prime}, x\right)=-\left(p q^{\prime}, x r^{\prime}, x\right)=\left(p q^{\prime}, x, r^{\prime}\right) x$

$\partial\left(p q^{\prime}\right)=\partial p+\partial q^{\prime}=1+(-1+\partial q)=\partial q$

$\partial x=1 \quad \partial r^{\prime}=-1+\partial r$

Assim: $\quad \partial\left(p q^{\prime}\right)+\partial x+\partial r^{\prime}=\partial q+1-1+\partial r=\partial q+\partial r<n$

Logo: $\quad\left(p q^{\prime}, x, r^{\prime}\right)=0 \quad$ e também $\quad\left(p q^{\prime}, x, r^{\prime}\right) x=0$

Para a segunda parcela temos:

$$
\begin{aligned}
& \left(x r^{\prime}, x, q^{\prime}\right) p=-\left(x r^{\prime}, q^{\prime}, x\right) p=\left(q^{\prime}, x r^{\prime}, x\right) p=\left(q^{\prime}, x, r^{\prime}\right) x p \\
& \partial q^{\prime}=-1+\partial q \quad \partial x=1 \quad \partial r^{\prime}=-1+\partial r
\end{aligned}
$$


Daí $\quad \partial q^{\prime}+\partial x+\partial r^{\prime}=-1+\partial q+1-1+\partial r=\partial q+\partial r-1<n$

Logo: $\quad\left(q^{\prime}, x, r^{\prime}\right)=0 \quad$ e também $\quad\left(q^{\prime}, x, r^{\prime}\right) x p=0$

Agora, a terceira parcela: $\left(x r^{\prime}, p, q^{\prime}\right) x$ Calculamos:

$\partial\left(x r^{\prime}\right)=\partial r \quad \partial x=1 \quad \partial q^{\prime}=-1+\partial q$

Então $\quad \partial\left(x r^{\prime}\right)+\partial p+\partial q^{\prime}=\partial r+1-1+\partial q=\partial p+\partial q<n$

Logo: $\quad\left(x r^{\prime}, p, q^{\prime}\right) x=0$

Como cada parcela se anula, temos que: $\quad(p, q, r)=0$

(2) $\partial q>1$ ou $\partial r>1$

Se apenas um dos produtos $q$ ou $r$ tem grau $>1$, digamos $\partial q>1, \quad q=x q^{\prime}$, temos, novamente pelo Corolário 1.2.3:

$(p, q, r)=\left(p, x q^{\prime}, x\right)=\left(p, x, q^{\prime}\right) x=0 \quad$ pelo mesmo argumento usado acima.

(3) $\partial q=\partial r=1$

Se $\partial q=\partial r=1$, pela lei alternativa à direita temos: $(p, q, r)=(p, x, x)=0$

O próximo resultado irá estabelecer uma importante relação entre as álgebras nilpotentes e as nilálgebras. Pelas definições concluímos que toda álgebra nilpotente é uma nilálgebra. De fato, se $A$ é nilpotente, então existe $n \in \mathbb{N}$ tal que $A^{(n)}=0$. Então, para todo $x \in A$, tem-se $x^{n}=0$, o que significa que $x$ é nilpotente. Portanto $A$ é uma nilálgebra.

A recíproca pode não ser, necessariamente, verdadeira. Mas em dimensão finita, se $A$ é nilálgebra, então $A$ é nilpotente. Vamos primeiro demonstrar este fato no caso associativo. Para álgebras alternativas precisaremos antes conceituar álgebra de multiplicação $M(A)$ de uma álgebra alternativa $A$, e em seguida demonstrar um lema pertinente a tais álgebras.

Os operadores $R_{a}$ e $L_{a}$, há pouco definidos são evidentemente lineares e, podemos considerar os conjuntos:

$R(A)$ de todos os operadores multiplicativos à direita de $A$;

$L(A)$ de todos os operadores multiplicativos à esquerda de $A$; 
B de todos os operadores lineares sobre $A$.

Claramente B é uma álgebra sobre $F$. Os conjuntos $R(A)$ e $L(A)$ são subespaços da álgebra associativa $\mathbf{B}$, uma vez que $R_{a}$ e $L_{a}$ são transformações lineares.

Definição 1.2.2. Chama-se álgebra envolvente das multiplicações à direita e ⿳亠㐅冋 esquerda de $A$, denotada por $M(A)$ (ou simplesmente $M$ ), a subálgebra de $\mathbf{B}$ gerada pela reunião dos operadores multiplicativos à direita e à esquerda de $A$.

$M$ é a intersecção de todas as subálgebras de $\mathbf{B}$ que contêm $R(A)$ e $L(A)$. Os elementos de $M$ são da forma $\sum S_{a_{1}} \ldots S_{a_{n}}$ onde $S_{a_{i}}$ é igual a $R_{a_{i}}$ ou $L_{a_{i}}$.

Seja $B \subset A$, qualquer subconjunto. Consideremos a álgebra envolvente de todos os operadores multiplicativos à direita e à esquerda de $A$, definidos por elementos de $B$, que será denotada por $B^{*}$.

Note que, se $A$ é uma álgebra e $B \subseteq A$, então, na notação acima introduzida temse: $A^{*}=M(A)$, mas se $B$ é uma subálgebra própria de $A$, então $B^{*} \neq M(B)$. De fato, $B^{*}$ é gerada por operadores $R_{b_{i}}, L_{b_{j}}$, com $b_{i}, b_{j} \in B$, calculados em elementos de $A$. Já $M(B)$ compõe-se dos mesmos operadores de $B^{*}$, porém calculados em elementos de $B$.

Lema 1.2.5. Um ideal $B$ de uma álgebra $A$ é nilpotente se e somente se a subálgebra $B^{*}$ de $M(A)$ é nilpotente.

Demonstração: $(\Rightarrow)$ Seja $B$ ideal nilpotente de $A$, com índice de nilpotência $n$. Sejam $T_{1}, \ldots, T_{n} \in B^{*}$. Cada $T_{i}$ pode ser escrito $T_{i}=f_{i}\left(S_{b_{1}}, \ldots, S_{b_{n}}\right), b_{i} \in B, i=$ $1, \ldots n$. Consideremos o produto: $T=T_{1} \ldots T_{n}=f_{1} \ldots f_{n}\left(S_{b_{1}}, \ldots, S_{b_{n}}\right)$. Se cada $T_{i}$ for um polinômio a apenas uma indeterminada $\left(S_{b_{i}}\right)$, $T$ será uma soma de produtos onde cada termo tem pelo menos $n$ operadores lineares $S_{b_{i}}=R_{b_{i}}$ ou $L_{b_{i}}$. Ao calcularmos $T(x), x \in A$, como $B$ é ideal de $A$, teremos $T(x) \in B$. Logo, $T(x)$ será uma soma em que cada parcela é o produto de pelo menos $n+1$ elementos. Mas qualquer produto de $n$ ou mais elementos em $B$ é nulo, pois $B$ é nilpotente de índice $n$. Assim, $T(x)=0$, para todo $x \in A$, ou seja, $T=0$. Portanto $B^{*}$ é nilpotente.

$(\Leftarrow)$ Seja $B$ subálgebra de $A$. Vamos verificar que qualquer produto de pelo menos $2^{t}$ elementos de $B$ pode ser escrito como $S_{1} \ldots S_{n}(b), S_{i} \in B^{*}, b \in B$. Faremos indução em $t$. 
Para $t=1$, é fácil ver que o produto de 2 elementos de $B$ pode ser escrito $S_{b_{1}}(b)$. De fato, $b b_{1}=b R_{b_{1}}$ e $b_{1} b=b L_{b_{1}}$. Seja agora um produto de mais de 2 elementos. Podemos considerar a última multiplicação a ser feita: $b c$, onde $c$ é o resultado da multiplicação dos primeiros elementos. Como ilustração, se tivermos o produto: $b_{1}\left(b_{2}\left(b_{1} b_{3}\right)\right)$, fazemos $b=b_{1}$ e $c=b_{2}\left(b_{1} b_{3}\right)$.

Nossa hipótese de indução será: O produto de pelo menos $2^{t}$ elementos de $B$ é da forma $b S_{1} \ldots S_{t}$, com $S_{i} \in B^{*}, b \in B$. Calculemos o produto de pelo menos $2^{t+1}$ elementos de $B$. Novamente teremos uma última multiplicação a ser feita, envolvendo dois fatores: um deles é o produto de pelo menos $2^{t}$ elementos de $B$ e o outro é $c \in B$. Assim, pela hipótese de indução podemos escrever:

$\mathrm{Ou}$

$$
\begin{aligned}
& c\left(b S_{1} \ldots S_{t}\right)=b S_{1} \ldots S_{t} L_{c}=b S_{1} \ldots S_{t+1} \\
& \left(b S_{1} \ldots S_{t}\right) c=b S_{1} \ldots S_{t} R_{c}=b S_{1} \ldots S_{t+1}
\end{aligned}
$$

Assim, se $t$ é o índice de nilpotência de $B^{*}$, qualquer produto de $2^{t}$ elementos de $B$ será zero. Portanto $B$ é nilpotente.

Lema 1.2.6. Seja $A \neq 0$ uma álgebra associativa de dimensão finita e $a \in A$. Então: $A a=A$ se e somente se $x a \neq 0$, para todo $x \neq 0, x \in A$.

Demonstração: $(\Rightarrow)$ Seja $A=\left\langle u_{1}, \ldots, u_{n}\right\rangle$, onde $\left\{u_{1}, \ldots, u_{n}\right\}$ é uma base de $A$. Assim, $\left\{u_{1} a, \ldots, u_{n} a\right\}$ é uma base de $A a$. Seja $x \in A$ tal que $x a=0$. Podemos escrever: $x=\sum_{i=1}^{n} \alpha_{i} x_{i}, \operatorname{logo} 0=x a=\sum_{i=1}^{n} \alpha_{i} x_{i} a$, e daí, $\alpha_{i}=0$, para todo $i$. Portanto $x=0$.

$(\Leftarrow)$ Basta mostrar que $\operatorname{dim} A a=\operatorname{dim} A=n$, isto é, $\left\{u_{1} a, \ldots, u_{n} a\right\}$ é l.i. Seja $\operatorname{dim} A=n \operatorname{com}\left\{u_{1}, \ldots, u_{n}\right\}$ uma base de $A$. Claramente $\left\{u_{1} a, \ldots, u_{n} a\right\}$ gera $A a$. Sejam $\alpha_{1}, \ldots, \alpha_{n} \in F$ tais que $\alpha_{1} u_{1} a+\cdots+\alpha_{n} u_{n} a=0$. Então $\left(\alpha_{1} u_{1}+\cdots+\alpha_{n} u_{n}\right) a=$ $0 \log \mathrm{o} \alpha_{1} u_{1}+\cdots+\alpha_{n} u_{n}=0$, por hipótese. Mas como $\left\{u_{1}, \ldots, u_{n}\right\}$ é base de $A$, segue que $\alpha_{i}=0$ e $\left\{u_{1} a, \ldots, u_{n} a\right\}$ é l.i. como queríamos.

Lema 1.2.7 (Albert). Qualquer nilálgebra associativa de dimensão finita sobre $F$ é nilpotente.

Demonstração: Seja $A$ uma nilálgebra associativa de dimensão finita. Faremos indução na dimensão de $A$. Seja $n=\operatorname{dim} A$. Se $n=1, A=\langle u\rangle$, para algum 
$u \in A$. Assim, qualquer elemento $x \in A$ pode ser escrito $x=\alpha_{1} u, \alpha_{1} \in F$. Mas, pela tábua de multiplicação de $A$, existe $\alpha \in F$ tal que $u^{2}=\alpha u$. Daí: $x, y \in A \Rightarrow x=\alpha_{1} u, y=\alpha_{2} u \Rightarrow x y=\alpha_{1} u \alpha_{2} u=\alpha_{1} \alpha_{2} u^{2}=\alpha_{1} \alpha_{2} \alpha u$. Podemos ter dois casos:

(1) $\alpha=0$. Teremos $x y=0, \forall x, y \in A$. Neste caso $A$ é uma zero álgebra;

(2) $\alpha \neq 0$. Temos $\left(\alpha^{-1} u\right)^{2}=\left(\alpha^{-1}\right)^{2} u^{2}=\alpha^{-1} u$. Fazendo $\alpha^{-1} u=e$, temos que $e^{2}=e \neq 0$, o que é uma contradição, pois $A$ é uma nilálgebra. Logo $A$ é uma zero álgebra, isto é, $A$ é nilpotente de índice 2 .

Supondo que a tese é válida para $\operatorname{dim} A<n$, então todo ideal próprio $I$ de $A$ é nilpotente, uma vez que $\operatorname{dim} I<\operatorname{dim} A=n$. Seja $a \in A$. Se $A a=0$, então $x a=$ $0, \forall x \in A$. Logo $A$ é uma zero álgebra e, portanto, nilpotente. Excluído este caso, se for $A a=A$, então, pelo lema anterior, $x a \neq 0, \forall x \in A, x \neq 0$. Em particular, $a^{2} \neq 0, \operatorname{logo} a^{n} \neq 0, \forall n$. Contradição, pois $A$ é nilálgebra. Assim, $A a$ é um ideal próprio de $A$. Seja $r$ o índice de nilpotência de $A a$. Então $(A a A)^{r}=(A a)^{r} A=0$. Logo $A a A$ é nilpotente. Mas $A^{3}=\sum_{i=1}^{n} A a_{i} A$, onde $\left\{a_{1}, \ldots, a_{n}\right\}$ é uma base de $A$. Logo $A^{3}$ é nilpotente e, portanto, também $A$ é nilpotente.

Lema 1.2.8. Sejam A uma álgebra alternativa e $x, y \in A$ elementos quaisquer. São válidas as seguintes igualdades dos operadores $R_{a}$ e $L_{a}$ :

$$
\begin{aligned}
R_{x} R_{y}-R_{x y} & =L_{x y}-L_{y} L_{x}=L_{y} R_{x}-R_{x} L_{y} \\
& =L_{x} L_{y}-L_{y x}=R_{y} L_{x}-L_{x} R_{y} \\
& =R_{y x}-R_{y} R_{x}
\end{aligned}
$$

Demonstração: Seja $z \in A$. Calculemos cada uma das expressões acima em $z$, e façamos a subtração membro a membro, usando as propriedades usuais dos operadores lineares:

$$
\begin{aligned}
z\left(R_{x} R_{y}-R_{x y}\right)-z\left(L_{x y}-L_{y} L_{x}\right) & =z R_{x} R_{y}-z R_{x y}-z L_{x y}+z L_{y} L_{x} \\
& =(z x) R_{y}-z(x y)-(x y) z+(y z) L_{x} \\
& =(z x) y-z(x y)-(x y) z+x(y z) \\
& =(z, x, y)-(x, y, z)=0
\end{aligned}
$$




$$
\begin{aligned}
& z\left(L_{x y}-L_{y} L_{x}\right)-z\left(L_{y} R_{x}-R_{x} L_{y}\right)=z L_{x y}-z L_{y} L_{x}-z L_{y} R_{x}-z R_{x} L_{y} \\
& =(x y) z-(y z) L_{x}-(y z) R_{x}-(z x) L_{y} \\
& =(x y) z-x(y z)-(y z) x-y(z x) \\
& =(x, y, z)-(y, z, x)=0 \\
& z\left(L_{y} R_{x}-R_{x} L_{y}\right)-z\left(L_{x} L_{y}-L_{y x}\right)=z L_{y} R_{x}-z R_{x} L_{y}-z L_{x} L_{y}+z L_{y x} \\
& =(y z) R_{x}-(z x) L_{y}-(x z) L_{y}+(y x) z \\
& =(y z) x-y(z x)-y(x z)+(y x) z \\
& =(y, z, x)+(y, x, z)=0 \\
& z\left(L_{x} L_{y}-L_{y x}\right)-z\left(R_{y} L_{x}-L_{x} R_{Y}\right)=z L_{x} L_{y}-z L_{y x}-z R_{y} L_{x}+z L_{x} R_{y} \\
& =(x z) L_{y}-(y x) z-(z y) L_{x}-(x z) R_{y} \\
& =y(x z)-(y x) z-x(z y)+(x z) y \\
& =-(y, x, z)+(x, z, y)=0 \\
& z\left(R_{y} L_{x}-L_{x} R_{y}\right)-z\left(R_{y x}-R_{y} R_{x}\right)=z R_{y} L_{x}-z L_{x} R_{y}-z R_{y x}+z R_{y} R_{x} \\
& =(z y) L_{x}-(x z) R_{y}-x(y x)+(z y) R_{x} \\
& =x(z y)-(x z) y-z(y x)+(z y) x \\
& =-(x, z, y)+(z, y, x)=0
\end{aligned}
$$

Teorema 1.2.9. Qualquer nilálgebra alternativa de dimensão finita sobre F é nilpotente.

Demonstração: Seja $B$ uma subálgebra própria de $A$, maximal em relação à propriedade que $B^{*}$ é nilpotente. O Teorema 1.2.4 (Artin) garante a existência de tal álgebra. Então existe $x \in A$ tal que $x \notin B$. Definimos o conjunto das imagens de $x$ pelos operadores de $B^{*}$, por:

$$
B^{*} x=\left\{G(x) \mid G \in B^{*}\right\}
$$


Temos: $B^{*} x \subseteq B$. De fato:

Como $B^{*}$ é nilpotente, $B^{*^{r}}=0$, para algum $r \in \mathbb{N}$. Então $A B^{*^{r}}=0 \subseteq B$.

Seja o conjunto $K=\left\{m \in \mathbb{N} \mid A B^{*^{m}} \subseteq B\right\}$. Tal conjunto não é vazio, pois $r \in K$. Sendo $K$ um conjunto de números naturais não vazio, o Princípio da Boa Ordem garante que $K$ tem um mínimo $m \geq 1$.

Assim, existe $m \in \mathbb{N}$ tal que $A B^{*^{m}} \subseteq B$ e $A B^{*^{m-1}} \nsubseteq B$. Se $m=1$, tome $x \in A \backslash B$. Se $m>1$, tome $x \in A B^{*^{m-1}}, x \notin B$. Com isto, obtemos $B^{*} x \subseteq B$.

Seja $C=B+F[x]$, onde $F[x]$ indica polinômios em $x$ com termo independente nulo. Claramente, $C$ é uma subálgebra de $A, \operatorname{com} B \subset C$. Consideremos a sua álgebra envolvente $C^{*}=(B+F[x])^{*}$. Mostremos inicialmente que $(B+F[x])^{*}=(B+F x)^{*}$.

Seja $c \in B+F[x]$. Então: $c=b+\sum_{j=1}^{n} \alpha_{j} x^{j}$, para algum $n$.

Se $S_{c} \in(B+F[x])^{*}$, então $S_{c}=L$ ou $S_{c}=R$, e escrevemos:

$$
\begin{aligned}
S_{c} & =S_{b}+\sum_{j \neq 0} \alpha_{j} S_{x^{j}}=S_{b}+\sum_{j \neq 0} \alpha_{j} S_{x}^{j} \Rightarrow S_{c} \in(B+F x)^{*} \\
& \Rightarrow(B+F[x])^{*} \subseteq(B+F x)^{*} .
\end{aligned}
$$

Por outro lado, se $c \in B+F x$, então $c=b+\alpha x$. Daí,

$$
\begin{aligned}
S_{c} & \in(B+F x)^{*} \Rightarrow S_{c}=S_{b}+\alpha S_{x} \Rightarrow S_{c} \in(B+F[x])^{*} . \\
& \Rightarrow(B+F x)^{*} \subseteq(B+F[x])^{*} . \quad \text { Portanto }(B+F[x])^{*}=(B+F x)^{*} .
\end{aligned}
$$

Um elemento $T \in C^{*}$ pode ser escrito $T=\sum S_{c_{1}} \ldots S_{c_{k}}$, onde $S_{c_{i}}=L_{c_{i}}$ ou $S_{c_{i}}=R_{c_{i}}$. Vamos procurar explicitar o elemento $T$ em termos dos operadores de $B^{*}$ e de $(F x)^{*}$. Consideremos as igualdades indicadas no Lema 1.2.8, fazendo $y=b$, para qualquer $b \in B$. Levando em conta que $B^{*} x \subseteq B$, temos:

Usando a igualdade $R_{x} R_{y}-R_{x y}=R_{y x}-R_{y} R_{x}$, resulta $R_{x} R_{b}-R_{x b}=R_{b x}-R_{b} R_{x} \Rightarrow R_{x} R_{b}=R_{b^{\prime}}+R_{b^{\prime \prime}}-R_{b} R_{x}$.

Considerando $b_{1}=b^{\prime}+b^{\prime \prime}$, temos: $R_{x} R_{b}=R_{b_{1}}-R_{b} R_{x}$.

Usando a igualdade $L_{y} R_{x}-R_{x} L_{y}=R_{y x}-R_{y} R_{x}$, vem

$$
L_{b} R_{x}-R_{x} L_{b}=R_{b x}-R_{b} R_{x} \Rightarrow R_{x} L_{b}=L_{b} R_{x}+R_{b} R_{x}-R_{b_{2}} .
$$

Da igualdade $R_{y} L_{x}-L_{x} R_{y}=L_{x y}-L_{y} L_{x}$, obtemos

$$
R_{b} L_{x}-L_{x} R_{b}=L_{x b}-L_{b} L_{x} \Rightarrow L_{x} R_{b}=R_{b} L_{x}+L_{b} L_{x}-L_{b_{3}} .
$$

Usando, finalmente $L_{x} L_{y}-L_{y x}=L_{x y}-L_{y} L_{x}$, obtemos

$$
L_{x} L_{b}-L_{b x}=L_{x b}-L_{b} L_{x} \Rightarrow L_{x} L_{b}=L_{b_{1}}-L_{b} L_{x} .
$$


Vemos assim que, em cada produto da multiplicação à direita e à esquerda em $B^{*}$ e $(F x)^{*}$, a multiplicação $R_{x}$ ou $L_{x}$ pode ser passada sistematicamente da esquerda para a direita de $R_{b}$ ou $L_{b}$ de modo que, embora possa mudar de sinal e introduzir novos termos, preserva o número de fatores de $B^{*}$, e não aumenta o número de fatores de $(F x)^{*}$. Portanto, todo operador $T \in C^{*}$ pode ser escrito como combinação linear de termos da forma:

$$
\begin{gathered}
R_{x}^{j_{1}}, \quad L_{x}^{j_{2}}, \quad R_{x}^{j_{3}} L_{x}^{j_{4}}, \quad B_{1}, \quad B_{2} R_{x}^{m_{1}}, \quad B_{3} L_{x}^{m_{2}}, \quad B_{4} R_{x}^{m_{3}} L_{x}^{m_{4}} \\
B_{i} \in B^{*}, \quad j_{i} \geq 1, \quad m_{i} \geq 1
\end{gathered}
$$

Seja $r$ o índice de nilpotência de $B^{*}$, isto é $B^{*^{r}}=0$. E como $A$ é nilálgebra o $x$ que tomamos em $A$ é nilpotente. Então existe $j \in \mathbb{N}$ tal que $x^{j}=0$.

Usando (1), (2), (3), (4) e (5), basta fazer $\left(T^{r}\right)^{2 j-1}=T^{r(2 j-1)}$ que teremos certamente $T^{r(2 j-1)}=0$, lembrando que $R_{x}$ e $L_{x}$ comutam.

Assim, todo elemento $\mathrm{T}$ da álgebra associativa de dimensão finita $C^{*}$ é nilpotente. Logo, pelo Lema 1.2.7, $C^{*}$ é nilpotente.

Mas $B$ é maximal em relação à propriedade que $B^{*}$ é nilpotente. Chegamos a esta contradição ao supormos $B$ subálgebra própria de $A$. Devemos ter, então, $B=A$, donde $A^{*}$ é nilpotente. Logo, pelo Lema 1.2.5, $A$ é nilpotente.

Corolário 1.2.10. Seja A álgebra alternativa de dimensão finita e nilpotente à direita (esquerda). Então A é nilpotente.

Demonstração: Se $A$ é nilpotente à direita (esquerda) então $A$ é uma nilálgebra. De fato, para todo $x \in A, x^{k}=0\left({ }^{k} x=0\right)$, onde $k$ é o índice de nilpotência de $A$. Logo $A$ é uma nilálgebra. Portanto, pelo Teorema 1.2.9, $A$ é nilpotente.

\subsection{Decomposição de Peirce}

O estudo das propriedades de uma álgebra fica facilitado se pudermos decompor esta álgebra em uma soma de álgebras mais convenientes. Uma das ferramentas mais importantes neste sentido é a decomposição de Peirce, que passamos a estudar.

Definição 1.3.1. Seja A uma álgebra alternativa. Um elemento e $\in A$ é chamado idempotente se $e \neq 0$, e $e^{2}=e$. 
O próximo lema, devido a Albert, juntamente com o Lema 1.2.6, permitem garantir a existência de idempotentes numa álgebra associativa de dimensão finita.

Lema 1.3.1 (Albert). Toda álgebra associativa de dimensão finita que não é uma nilálgebra possui um idempotente.

Demonstração: É imediato para álgebras de dimensão 1. Fazemos indução em $n=$ $\operatorname{dim} A$. Supondo válido se $\operatorname{dim} A<n$, seja $A a \subseteq A$, subálgebra, para algum $a \in A$. Pela hipótese de indução, $A a$ possui um idempotente se $A a$ for um ideal próprio de $A$ e o resultado está mostrado. Se $A a=A$, pelo Lema 1.2.6, $x a=0 \Rightarrow x=0$. Em particular, $a=e a$ para algum $e \in A$. Logo $e a=e^{2} a \Rightarrow\left(e^{2}-e\right) a=0 \Rightarrow e^{2}-e=$ $0 \Rightarrow e^{2}=e$. Portanto $e \in A$ é idempotente.

Os dois próximos lemas garantem a existência de idempotentes numa álgebra de potências associativas e numa álgebra alternativa, respectivamente.

Lema 1.3.2. Toda álgebra $A$ de potências associativas e de dimensão finita que não é uma nilálgebra, contém um idempotente e.

Demonstração: Como $A$ não é uma nilálgebra, existe $x \in A$ que não é nilpotente. Pelo Teorema 1.2.4 (Artin), a subálgebra $A(x)$ de $A$ é uma álgebra associativa de dimensão finita (pois $\operatorname{dim} A<\infty)$, que não é uma nilálgebra $(x \in A(x)$ e $x$ não é nilpotente). Logo, pelo Lema 1.3.1 (Albert), $A(x)$ contém um idempotente. Portanto $A$ contém um idempotente.

Lema 1.3.3. Toda álgebra alternativa $A$ de dimensão finita que não é uma nilálgebra, contém um idempotente e.

Demonstração: É fácil ver que toda álgebra alternativa é de potências associativas. Logo, pelo lema anterior, toda álgebra alternativa de dimensão finita que não é uma nilálgebra possui um idempotente.

Seja $A$ uma álgebra alternativa, e $e \in A$ um idempotente. $A$ pode ser decomposta numa soma direta de subespaços vetoriais:

$$
\begin{gathered}
A=A_{11} \oplus A_{10} \oplus A_{01} \oplus A_{00}, \text { onde } \\
A_{i j}=\left\{x_{i j} \mid e x_{i j}=i x_{i j}, \quad x_{i j} e=j x_{i j}\right\} \quad i, j=0,1
\end{gathered}
$$


A seguinte decomposição de um elemento $x$ qualquer de uma álgebra alternativa $A$ em relação a um idempotente $e$ é bastante útil:

$$
x=\underbrace{e x e}_{(1)}+\underbrace{(e x-e x e)}_{(2)}+\underbrace{(x e-e x e)}_{(3)}+\underbrace{(x-e x-x e+e x e)}_{(4)}
$$

Mostremos que cada uma das parcelas indicadas está em um dos subespaços $A_{i j}$ da decomposição de Peirce.

(1) exe $\in A_{11}$.

De fato, usando as propriedades usuais das álgebras alternativas, temos:

$$
\begin{aligned}
& e(e x e)=-(e, e, x e)+e^{2}(x e)=0+e(x e)=e x e=1 \text { exe } \\
& (e x e) e=(e x, e, e)+(e x) e^{2}=0+e x e=e x e=1 e x e
\end{aligned}
$$

(2) $($ ex - exe $) \in A_{10}$.

Seja $y=e x-e x e$. Mostremos que $e y=y$ e que $y e=0$ :

$$
\begin{aligned}
& e y=e(e x-e x e)=e(e x)-e(e x e)=e x-e x e=y \\
& y e=(e x-e x e) e=e x e-(e x e) e=e x e-e x e^{2}=e x e-e x e=0
\end{aligned}
$$

(3) $(x e-e x e) \in A_{01}$.

Fazendo $y=x e-e x e$, mostremos que $e y=0$ e que $y e=y$ :

$$
\begin{aligned}
& e y=e(x e-e x e)=e x e-e(e x e)=e x e-e^{2} x e=e x e-e x e=0 \\
& y e=(x e-e x e) e=(x e) e-(e x e) e=x e^{2}-e x e^{2}=x e-e x e=y
\end{aligned}
$$

(4) $(x-e x-x e+e x e) \in A_{00}$

Basta mostrar que $e y=y e=0$, onde $y=x-e x-x e+e x e$

$$
\begin{aligned}
e y=e(x-e x-x e+e x e) & =e x-e(e x)-e(x e)+e(e x e) \\
& =e x-e^{2} x-e x e+e^{2} x e \\
& =e x-e x-e x e+e x e=0
\end{aligned}
$$

Definição 1.3.2. Em uma álgebra alternativa $A$, os idempotentes $e_{1}, e_{2}, \ldots, e_{t}$ são chamados dois a dois ortogonais se $e_{i} e_{j}=0, \quad i \neq j$.

Lema 1.3.4. Em uma álgebra alternativa $A$, com $e_{1}, e_{2}, \ldots, e_{t}$ idempotentes dois a dois ortogonais, tem-se:

(i) $e=e_{1}+e_{2}+\cdots+e_{t}$ é um idempotente 
(ii) $e e_{i}=e_{i} e=e_{i}, \quad \forall i$

(iii) $\left(x, e_{i}, e_{j}\right)=\left(x, e_{i}, e\right)=0, \quad \forall x \in A$

\section{Demonstração:}

(i) Calculemos $e^{2}=\left(e_{1}+e_{2}+\cdots+e_{t}\right)\left(e_{1}+e_{2}+\cdots+e_{t}\right)=e_{1}^{2}+\cdots+e_{t}^{2}+\sum_{i \neq j} e_{i} e_{j}$ Como os $e_{i}$ são idempotentes, $e_{i} e_{j}=0$ se $i \neq j$. Resulta então: $e^{2}=e_{1}^{2}+\cdots+e_{t}^{2}=e_{1}+e_{2}+\cdots+e_{t}=e$

(ii) $e e_{i}=\left(e_{1}+e_{2}+\cdots+e_{t}\right) e_{i}=e_{i}^{2}=e_{i}, \quad \forall i$

(iii) Se $i=j$ resulta imediatamente que $\left(x, e_{i}, e_{i}\right)=0$. Seja $i \neq j$ :

$$
\begin{aligned}
& \left(x, e_{1}, e_{j}\right)=\left(x e_{i}\right) e_{j}-x\left(e_{i} e_{j}\right)=\left(x e_{i}\right) e_{j}, \text { pois } e_{i} e_{j}=0 . \\
& \left(x e_{i}\right) e_{j}=\left(x e_{i}\right) e_{j}^{2}=\left(x, e_{i}, e_{j}\right) e_{j}=-\left(x, e_{j}, e_{i}\right) e_{j}=-\left(\left(x e_{j}\right) e_{i}\right) e_{j} \\
& \quad \stackrel{(*)}{=}-x\left(e_{j} e_{i} e_{j}\right)=0
\end{aligned}
$$

(*) Moufang, identidade (iii)

Definição 1.3.3. Um idempotente e de uma álgebra A é chamado primitivo se não existem idempotentes ortogonais u e $v$ em $A$ tais que $e=u+v$.

Lema 1.3.5. Em uma álgebra $A$ de dimensão finita, qualquer idempotente e pode ser escrito como $e=e_{1}+\cdots+e_{t}$, com $e_{i}(i=1, \ldots, t)$ idempotentes primitivos dois a dois ortogonais.

Demonstração: Se e é primitivo nada temos a mostrar. Se $e$ não é primitivo, existem $u$ e $v$ tais que $e=u+v$. Se $u$ e $v$ forem primitivos, está demonstrado. Caso contrário, teremos $e=u_{1}+\cdots+u_{r}$. Os idempotentes $u_{i}$ geram um subespaço de dimensão $r$, que é uma subálgebra associativa e comutativa, $\operatorname{com} r \leq \operatorname{dim} A$. Se ainda algum $u_{i}$ não for primitivo, podemos decompô-lo em uma soma. Como $A$ tem dimensão finita, o processo deve se encerrar e chegamos a $e=e_{1}+\cdots+e_{t}$, com os $e_{i}$ idempotentes primitivos dois a dois ortogonais.

Pode-se obter uma decomposição de Peirce mais refinada considerando-se os idempotentes dois a dois ortogonais $e_{1}, \ldots, e_{t}$. Tal decomposição é dada por:

$$
A=\sum_{i, j} A_{i j} \quad i, j=0,1, \ldots t
$$


onde, usando o $\delta$ de Kronecker, definimos:

$$
\begin{gathered}
A_{i j}=\left\{x_{i j} \mid e_{k} x_{i j}=\delta_{k i} x_{i j}, \quad x_{i j} e_{k}=\delta_{j k} x_{i j}, \quad k=0,1, \ldots, t\right\} \\
i, j=0,1, \ldots t
\end{gathered}
$$

Apresentamos a seguir uma série de propriedades da decomposição de Peirce, as quais terão grande utilidade no próximo capítulo quando trataremos do radical de uma álgebra.

Proposição 1.3.6 (Existência e Unicidade da Decomposição de Peirce). Seja A uma álgebra alternativa, e $e_{1}, \ldots, e_{t}$ idempotentes dois a dois ortogonais. Então: $A=\sum_{i, j} A_{i j}, \quad i, j=0,1, \ldots, t, \quad$ onde:

$$
\begin{gathered}
A_{i j}=\left\{x_{i j} \mid e_{k} x_{i j}=\delta_{k i} x_{i j}, \quad x_{i j} e_{k}=\delta_{j k} x_{i j}, \quad k=0,1, \ldots, t\right\} \\
i, j=0,1, \ldots t .
\end{gathered}
$$

Tal decomposição é única.

Demonstração: Seja $x \in \sum A_{i j}$, então $x=\sum_{k, l=0}^{t} x_{k l}$

Comecemos calculando $e_{i} x e_{j}$, para $i=1, \ldots, t$.

$$
e_{i} x e_{j}=e_{i}\left(\sum_{k, l=0}^{t} x_{k l}\right) e_{j}=\sum_{k, l=0}^{t} e_{i} x_{k l} e_{j}=\sum_{k l}^{t} \delta_{i k} x_{k l} e_{j}=\sum_{k l}^{t} \delta_{i k} \delta_{l j} x_{k l}=x_{i j}
$$

Observação: Podemos escrever $e_{i} x e_{j}$, pois $\left(e_{i} x\right) e_{j}=e_{i}\left(x e_{j}\right)$, já que $\left(x, e_{i}, e_{j}\right)=0$, conforme Lema 1.3.4.

Encontramos, então:

$$
e_{i} x e_{j}=x_{i j}
$$

Calculemos agora $e_{i} x$ e $e_{i} x e$, para $i=1, \ldots, t$.

$$
\begin{gathered}
e_{i} x=e_{i} \sum_{k, l=0}^{t} x_{k l}=\sum_{k, l=0}^{t} e_{i} x_{k l}=\sum_{k, l=0}^{t} \delta_{i k} x_{k l}=\sum_{l=0}^{t} x_{i l} \\
e_{i} x e=e_{i} x \sum_{k=1}^{t} e_{k}=\sum_{l=0}^{t} x_{i l} \sum_{k=1}^{t} e_{k}=\sum_{l=0}^{t} \sum_{k=1}^{t} x_{i l} e_{k}=\sum_{l=0}^{t} \sum_{k=1}^{t} \delta_{l k} x_{i l}=\sum_{l=1}^{t} x_{i l}
\end{gathered}
$$


Observação: Note que este último somatório começa mesmo em $l=1$, pois para $l=0, \quad \delta_{0 k}=0$, para todo $k=1, \ldots, t$.

Podemos escrever, então:

$$
e_{i} x-e_{i} x e=x_{i 0}
$$

De maneira análoga, calculando $x e_{j}$, e $e x e_{j}$, obtemos:

$$
\begin{gathered}
x e_{j}=\left(\sum_{k, l=0}^{t} x_{k l}\right) e_{j}=\sum_{k, l=0}^{t} x_{k l} e_{j}=\sum_{k, l=0}^{t} \delta_{l j} x_{k l}=\sum_{k=0}^{t} \sum_{l=0}^{t} \delta_{l j} x_{k l} \\
=\sum_{k=0}^{t}(\overbrace{\delta_{0 j} x_{k 0}+\delta_{1 j} x_{k 1}}^{=0}+\cdots+\overbrace{\delta_{j j} x_{k j}}^{=x_{k j}}+\cdots+\overbrace{\delta_{l j} x_{k l}}^{=0}) \\
=\sum_{k=0}^{t} \delta_{j j} x_{k j}=\sum_{k=0}^{t} x_{k j} \\
\operatorname{exe}_{j}=\left(\sum_{l=1}^{t} e_{l}\right)\left(\sum_{k=0}^{t} x_{k j}\right)=\sum_{l=1}^{t} e_{l} \overbrace{k=0}^{t} e_{l} x_{k j}=\sum_{l=1}^{t} e_{l} \sum_{k=0}^{t} \delta_{l k} x_{k j} \\
=\sum_{k=0}^{t} \sum_{l=1}^{t} \delta_{l k} x_{k j}=\sum_{k=0}^{t}(\overbrace{\delta_{1 k} x_{k j}}^{=0}+\cdots+\overbrace{\delta_{k k} x_{k j}}^{t}+\cdots+\overbrace{\delta_{k t} x_{k j}}^{t})=\sum_{k=1}^{t} x_{k j}
\end{gathered}
$$

Temos assim:

$$
x e_{j}-e x e_{j}=x_{0 j}
$$

Falta encontrar uma expressão para $x_{00}$, que podemos escrever em função das parcelas já calculadas, como:

$$
\begin{gathered}
x_{00}=x-\sum_{i, j=1}^{t} x_{i j}-\sum_{i=1}^{t} x_{i 0}-\sum_{j=1}^{t} x_{0 j} \\
=x-\sum_{i, j=1}^{t} e_{i} x e_{j}-\sum_{i=1}^{t}\left(e_{i} x-e_{i} x e\right)-\sum_{j=1}^{t}\left(x e_{j}-e x e_{j}\right) \\
=x-e x e-(e x-e x e)-(x e-e x e)=x-e x e-e x+e x e-x e+e x e
\end{gathered}
$$

Resulta, finalmente:

$$
x_{00}=x-e x-x e+e x e
$$

Dado $x \in A$, então:

$$
x=\sum_{i, j=1}^{t} x_{i j}+\sum_{i=1}^{t} x_{i 0}+\sum_{j=1}^{t} x_{0 j}+x_{00} \in \sum A_{i j} \text {, onde } x_{i j}=e_{i} x e_{j},
$$


$x_{i 0}=e_{i} x-e_{i} x e, \quad x_{0 j}=x e_{j}-e x e_{j} \quad$ e $\quad x_{00}=x-e x-x e+e x e$

Portanto: $A \subset \sum_{i, j=0}^{t} A_{i j}$. Como, claramente $\sum_{i, j=0}^{t} A_{i j} \subset A$, segue que: $A=\sum_{i, j=0}^{t} A_{i j}$.

Mostremos que a decomposição é única. Vimos que:

$$
x_{i j}=e_{i} x e_{j}, \quad i, j=1, \ldots, t
$$

donde $x=\sum x_{i j}$

Se houvesse outra decomposição: $x=\sum y_{i j}$, então:

$$
y_{i j}=e_{i} x e_{j}=x_{i j}, i, j=1, \ldots, t
$$

Analogamente para os outros casos.

Proposição 1.3.7 (Propriedades da Decomposição de Peirce). A decomposição de Peirce, conforme indicada na proposição acima, satisfaz as seguintes propriedades:

1. $A_{i j} A_{j k} \subseteq A_{i k} \quad(i, j, k=0,1, \ldots, t)$

2. $A_{i j} A_{i j} \subseteq A_{j i} \quad(i, j=0,1, \ldots, t)$

3. $A_{i j} A_{k l}=0 \quad j \neq k,(i, j) \neq(k, l) \quad(i, j, k, l=0,1, \ldots, t)$

4. $x_{i j}^{2}=0 \quad \forall x_{i j} \in A_{i j} \quad(i \neq j)$

5. $x_{i j} y_{i j}=-y_{i j} x_{i j} \quad \forall x_{i j}, y_{i j} \in A_{i j} \quad(i \neq j)$

6. $\left(x_{i j}, y_{j k}, z_{k i}\right)=0 s e(i, j, k) \neq(i, i, i), \forall x_{i j} \in A_{i j}, y_{j k} \in A_{j k}, z_{k i} \in A_{k i}$

7. $\left(x_{i i}, y_{i j} z_{j i}, t_{i i}\right)=0$ se $i \neq j, \quad \forall x_{i i}, t_{i i} \in A_{i i}, \quad y_{i j} \in A_{i j}, \quad z_{j i} \in A_{j i}$

8. $\left(x_{i j} y_{i j}\right) z_{i j}=\left(y_{i j} z_{i j}\right) x_{i j}=\left(z_{i j} x_{i j}\right) y_{i j}$ se $i \neq j, \quad \forall x_{i j}, y_{i j}, z_{i j} \in A_{i j}$

9. $x_{i j}\left(y_{i j} z_{j j}\right)=\left(x_{i j} z_{j j}\right) y_{i j}=z_{j j}\left(x_{i j} y_{i j}\right)$ se $i \neq j, \forall x_{i j}, y_{i j} \in A_{i j}, z_{j j} \in A_{j j}$

10. $x_{i j}\left(z_{i i} y_{i j}\right)=\left(z_{i i} x_{i j}\right) y_{i j}=\left(x_{i j} y_{i j}\right) z_{i i}$ se $i \neq j, \forall x_{i j}, y_{i j} \in A_{i j}, z_{i i} \in A_{i i}$

11. $\left(x_{i i} a\right)^{m}=\left(x_{i i} a_{i i}\right)^{m-1} \sum_{k=0}^{t} x_{i i} a_{i k}$

12. $\left(x_{i j} a\right)^{m}=\left(x_{i j} a_{j i}\right)^{m-1} \sum_{k=0}^{t} x_{i j} a_{j k}+\left(x_{i j} a_{j i}\right)\left(x_{i j} a_{j i}\right)^{m-1}, \quad$ se $i \neq j$ 
13. $e_{i}\left(x_{i j} a\right)^{m} e_{i}=\left(x_{i j} a_{j i}\right)^{m} \quad(i, j=0,1, \ldots, t)$

Demonstração: Omitiremos algumas demonstrações, já que os procedimentos são análogos para todas as propriedades.

1. $A_{i j} A_{j k} \subseteq A_{i k} \quad(i, j, k=0,1, \ldots, t)$

Seja $z \in A_{i j} A_{j k}$, isto é, $z=x_{i j} y_{j k}$. Calculemos $e_{l} z$ e $z e_{l}$ :

$$
\begin{aligned}
e_{l} z & =e_{l}\left(x_{i j} y_{j k}\right)=-\left(e_{l}, x_{i j}, y_{j k}\right)+\left(e_{l} x_{i j}\right) y_{j k}=\left(x_{i j}, e_{j}, y_{j k}\right)+\delta_{l i} x_{i j} y_{j k} \\
& =\left(x_{i j} e_{l}\right) y_{j k}-x_{i j}\left(e_{l} y_{j k}\right)+\delta_{l i} x_{i j} y_{j k}=\delta_{j l} y_{j k}-\delta_{l j} x_{i j} y_{j k}+\delta_{l i} x_{i j} y_{j k} \\
& =\delta_{l i} x_{i j} y_{j k}=\delta_{l i} z \\
z e_{l} & =\left(x_{i j} y_{j k} e_{l}=\left(x_{i j}, y_{j k}, e_{l}\right)+x_{i j}\left(y_{j k} e_{l}=-\left(x_{i j}, e_{l}, y_{j k}\right)+\delta_{k l} x_{i j} y_{j k}\right.\right. \\
& =\left(x_{i j}\left(e_{l} y_{j k}\right)-\left(x_{i j} e_{l}\right) y_{j k}+\delta_{k l} x_{i j} y_{j k}=\delta_{l j} x_{i j} y_{j k}-\delta_{j l} x_{i j} y_{j k}+\delta_{k l} x_{i j} y_{j k}\right. \\
& =\delta_{k l} x_{i j} y_{j k}=\delta_{k l} z \\
\Rightarrow z &
\end{aligned}
$$

3. $A_{i j} A_{k l}=0 \quad j \neq k, \quad(i, j) \neq(k, l) \quad(i, j, k, l=0,1, \ldots, t)$

Sejam $x_{i j} \in A_{i j}$ e $y_{k l} \in A_{k l}$. Basta mostrar que $x_{i j} y_{k l}=0$. Devemos considerar 3 casos:

(i) $l, k \neq 0, \quad l \neq k, \quad l \neq j$

Pelo Corolário 1.2.3, temos:

$$
(y, x a, z)+(y, z a, x)=-(y, x, a) z-(y, z, a) x
$$

Expandindo os associadores de (I):

$$
\begin{gathered}
(y(x a)) z-y((x a) z)+(y(z a)) x-y((z a) x)=(y(x a)-(y x) a) z+(y(z a)-(y z) a) x \\
=(y(x a)) z-((y x) a) z+(y(z a)) x-((y z) a) x \\
\Rightarrow y((x a) z)+y((z a) x)=((y x) a) z+((y z) a) x
\end{gathered}
$$

Fazendo em (II) as seguintes substituições: $y=x_{i j}, \quad x=e_{k}, \quad a=y_{k l} \quad$ e $z=e_{l}$ obtemos:

$$
\underbrace{x_{i j}\left(e_{k} y_{k l} e_{l}\right)}_{(1)}+\underbrace{x_{i j}\left(e_{l} y_{k l} e_{k}\right)}_{(2)}=\underbrace{\left(\left(x_{i j} e_{k}\right) y_{k l}\right) e_{l}}_{(3)}+\underbrace{\left(\left(x_{i j} e_{l}\right) y_{k l}\right) e_{k}}_{(4)}
$$


$(1) \rightarrow x_{i j}\left(e_{k} y_{k l} e_{l}\right)=x_{i j}\left(e_{k} y_{k l}\right) e_{l}=x_{i j}\left(\delta_{k k} y_{k l} e_{l}\right)=x_{i j}\left(\delta_{k k} \delta_{l l} y_{k l}\right)=x_{i j} y_{k l}$

$(2) \rightarrow x_{i j}\left(e_{l} y_{k l} e_{k}\right)=x_{i j}\left(e_{l} y_{k l}\right) e_{k}=\delta_{l k} y_{k l} e_{k}=0$

$(3) \rightarrow\left(\left(x_{i j} e_{k}\right) y_{k l}\right) e_{l}=\left(\delta_{j k} x_{i j} y_{k l}\right) e_{l}=0$

$(4) \rightarrow\left(\left(x_{i j} e_{l}\right) y_{k l}\right) e_{k}=\left(\delta_{j l} x_{i j} y_{k l}\right) e_{k}=0$

Portanto $x_{i j} y_{k l}=0$.

(ii) $l \neq k, \quad l=j$

A expressão (III) se reduz a:

$$
\begin{aligned}
x_{i j} y_{k j} & =\left(\delta_{l l} x_{i j} y_{k j}\right) e_{k}=\left(x_{i j} y_{k j}\right) e_{k}=\left(x_{i j}, y_{k j}, e_{k}\right)+x_{i j}\left(y_{k j} e_{k}\right) \\
& =\left(y_{k j}, e_{k}, x_{i j}\right)+\delta_{j k} x_{i j} y_{k l}=\left(y_{k j}, e_{k}, x_{i j}\right) \\
& =\left(y_{k j} e_{k} x_{i j}-y_{k j}\left(e_{k} x_{i j}\right)=\delta_{j k} y_{k j} x_{i j}-\delta_{k i} y_{k j} x_{i j}=0\right.
\end{aligned}
$$

pois $\delta_{j k}=0$, já que $j \neq k$. Também $\delta_{k i}=0$, pois como $l=j$, devemos ter, necessariamente, $i \neq k$, pois $(i, j) \neq(k, l)$.

(iii) $l=k$

Incluímos dois subcasos bem simples, e ficamos com:

(a) $j=l$

$$
x_{i j} y_{j j}+x_{i j} y_{j j}=\left(x_{i j} y_{j j}\right) e_{j} \Rightarrow 2 x_{i j} y_{j j}=x_{i j} y_{j j} \Rightarrow x_{i j} y_{j j}=0
$$

Usamos que, $x_{i j} y_{j j} e_{j}=z_{i j} e_{j}=\delta_{j j} z_{i j}=z_{i j}$, pois, pela propriedade 1 , $x_{i j} y_{j j} \in A_{i j}$.

(b) $j \neq l$

$$
x_{i j} y_{l l}+x_{i j} y_{l l}=0 \Rightarrow x_{i j} y_{l l}=0
$$

4. $x_{i j}^{2}=0 \quad \forall x_{i j} \in A_{i j} \quad(i \neq j)$

Pela propriedade $2, x_{i j}^{2} \in A_{j i}$. Como $i \neq j(\Rightarrow i \neq 0$ ou $j \neq 0)$, podemos supor $i \neq 0$. Então:

$$
x_{i j}^{2}=x_{i j}^{2} e_{i}=\left(x_{i j} x_{i j}\right) e_{i}=x_{i j}\left(x_{i j} e_{i}\right)=x_{i j}\left(\delta_{j i} x_{i j}\right)=0
$$

5. $x_{i j} y_{i j}=-y_{i j} x_{i j} \quad \forall x_{i j}, y_{i j} \in A_{i j} \quad(i \neq j)$

$$
\begin{gathered}
0=\left(x_{i j}+y_{i j}\right)^{2}=x_{i j}^{2}+x_{i j} y_{i j}+y_{i j} x_{i j}+y_{i j}^{2} \Rightarrow x_{i j} y_{i j}+y_{i j} x_{i j}=0 \\
\Rightarrow x_{i j} y_{i j}=-y_{i j} x_{i j}
\end{gathered}
$$


6. $\left(x_{i j}, y_{j k}, z_{k i}\right)=0$ se $(i, j, k) \neq(i, i, i), \forall x_{i j} \in A_{i j}, y_{j k} \in A_{j k}, z_{k i} \in A_{k i}$

$$
\left(x_{i j}, y_{j k}, z_{k i}\right)=-\left(y_{j k}, x_{i j}, z_{k i}\right)=-\underbrace{\left(y_{j k} x_{i j} z_{k i}\right)}_{(1)}+\underbrace{y_{j k}\left(x_{i j} z_{k i}\right)}_{(2)}
$$

$(1) \rightarrow$ Se $k \neq i, \quad y_{j k} x_{i j}=0$, pela propriedade 3 .

Se $k=i$, devemos ter $i \neq j$, logo $y_{j k} x_{i j}=y_{j i} x_{i j} \in A_{j j}$. Assim, $y_{j k} x_{i j}=w_{j j}$. Daí, $\left(y_{j k} x_{i j}\right) z_{k i}=w_{j j} z_{k i}=0$, pela prop. 3 , já que $i \neq j$ e $j \neq k$.

$(2) \rightarrow$ Se $j \neq k, \quad x_{i j} z k i=0$, pela propriedade 3 .

Se $j=k$ ambos devem ser $\neq i, \log 0 x_{i j} z_{k i}=x_{i j} z_{j i} \in A_{i i}$, e $x_{i j} z_{k i}=w_{i i}$.

Daí, $y_{j k} w_{i i}=y_{j j} w_{i i}=0$, pela prop. 3 .

7. $\left(x_{i i}, y_{i j} z_{j i}, t_{i i}\right)=0$ se $i \neq j, \quad \forall x_{i i}, t_{i i} \in A_{i i}, \quad y_{i j} \in A_{i j}, \quad z_{j i} \in A_{j i}$

$$
\begin{aligned}
& \left(x_{i i}, y_{i j} z_{j i}, t_{i i}\right)=\left(x_{i i}\left(y_{i j} z_{j i}\right)\right) t_{i i}-x_{i i}\left(\left(y_{i j} z_{j i}\right) t_{i i}\right) \\
& \quad=\left(-\left(x_{i i}, y_{i j}, z_{j i}\right)+\left(x_{i i} y_{i j}\right) z_{j i}\right) t_{i i}-x_{i i}\left(\left(y_{i j}, z_{j i}, t_{i i}\right)+y_{i j}\left(z_{j i}, t_{i i}\right)\right)
\end{aligned}
$$

Pela propriedade $6,\left(x_{i i}, y_{i j} z_{j i}\right)=0$ e $\left(y_{i j}, z_{j i}, t_{i i}\right)=0$, logo:

$$
\begin{aligned}
& \left(x_{i i}, y_{i j} z_{j i}, t_{i i}\right)=\left(\left(x_{i i} y_{i j}\right) z_{j i}\right) t_{i i}-x_{i i}\left(y_{i j}\left(z_{j i} t_{i i}\right)\right) \\
& \quad=\left(x_{i i} y_{i j}, z_{j i}, t_{i i}\right)+\left(x_{i i} y_{i j}\right)\left(z_{j i} t_{i i}\right)+\left(x_{i i}, y_{i j}, z_{j i} t_{i i}\right)-\left(x_{i i} y_{i j}\right)\left(z_{j i}, t_{i i}\right) \\
& \quad=\left(w_{i j}, z_{j i}, t_{i i}\right)+\left(x_{i i}, y_{i j} u_{j i}\right)=0
\end{aligned}
$$

Usei que: $x_{i i} y_{i j} \in A_{i j}, \log x_{i i} y_{i j}=w_{i j}$ e $z_{j i} t_{i i} \in A_{j i}$, logo $z_{j i} t_{i i}=u_{j i}$, de acordo com a propriedade 1 .

Pela prop. 6, os dois associadores no final da igualdade acima são iguais a zero.

8. $\left(x_{i j} y_{i j}\right) z_{i j}=\left(y_{i j} z_{i j}\right) x_{i j}=\left(z_{i j} x_{i j}\right) y_{i j}$ se $i \neq j, \quad \forall x_{i j}, y_{i j}, z_{i j} \in A_{i j}$

$$
\begin{aligned}
\left(x_{i j} y_{i j}\right) z_{i j} & =\left(x_{i j}, y_{i j}, z_{i j}\right)+x_{i j}\left(y_{i j} z_{i j}\right)=-\left(x_{i j}, z_{i j}\right) y_{i j}+x_{i j}\left(y_{i j} z_{i j}\right) \\
& =x_{i j}\left(z_{i j} y_{i j}\right)-\left(x_{i j} z_{i j}\right) y_{i j}+x_{i j}\left(y_{i j} z_{i j}\right) \\
& -x_{i j}\left(y_{i j} z_{i j}\right)+\left(z_{i j} x_{i j}\right) y_{i j}+x_{i j}\left(y_{i j} z_{i j}\right) \\
\Rightarrow\left(x_{i j} y_{i j}\right) z_{i j} & =\left(z_{i j} x_{i j}\right) y_{i j}
\end{aligned}
$$

A outra igualdade se demonstra de forma inteiramente análoga. 
11. $\left(x_{i i} a\right)^{m}=\left(x_{i i} a_{i i}\right)^{m-1} \sum_{k=0}^{t} x_{i i} a_{i k}$

Demonstramos por indução em $m$.

Para $m=1: \quad x_{i i} a=x_{i i} \sum_{j, k} x_{i i} a_{j k}=\sum_{k} x_{i i} a_{i k}$, pois $x_{i i} a_{j k}=0$ se $i \neq j \quad$ pela prop. 3.

Supondo válido para $m$, calculamos:

$$
\begin{aligned}
&\left(x_{i i} a\right)^{m+1}=\left(x_{i i} a\right)^{m}\left(x_{i i} a\right)=\left(x_{i i} a_{i i}\right)^{m-1}\left(\sum_{k=0}^{t} x_{i i} a_{i k}\right)\left(x_{i i} a\right) \\
&=\left(x_{i i} a_{i i}\right)^{m-1} \sum_{k=0}^{t}\left(x_{i i} a_{i k}\right)\left(x_{i i} a\right) \\
&=\left(x_{i i} a_{i i}\right)^{m-1}\left(\sum_{k=0}^{t}\left(x_{i i} a_{i k}\right) \sum_{j=0}^{t}\left(x_{i i} a_{i j}\right)\right) \\
&=\left(x_{i i} a_{i i}\right)^{m-1}(\sum_{k=0}^{t} \sum_{j=0}^{t} \underbrace{\left(x_{i i} a_{i k}\right)}_{(1)} \underbrace{\left(x_{i i} a_{i j}\right)}_{(2)}) \\
&=\left(x_{i i} a_{i i}\right)^{m-1} \sum_{j=0}^{t}\left(x_{i i} a_{i i}\right)\left(x_{i i} a_{i j}\right)=\left(x_{i i} a_{i i}\right)^{m} \sum_{j=0}^{t}\left(x_{i i} a_{i j}\right) \\
&(1) \rightarrow x_{i i} a_{i k} \in A_{i k}, \quad \mathrm{e} \quad(2) \rightarrow x_{i i} a_{i j} \in A_{i j} \Rightarrow\left(x_{i i} a_{i k}\right)\left(x_{i i} a_{i j}\right)=0
\end{aligned}
$$

se $k \neq i$, portanto o somatório em $k$ se anula para todo $k \neq i$, ficando apenas o termo $x_{i i} a_{i i}$.

\section{4 Álgebras Báricas}

As álgebras báricas têm sua origem nos estudos da Genética, e são caracterizadas por uma função peso relacionada às características genéticas transmitidas entre os indivíduos. Nosso estudo aqui visa somente os aspectos algébricos desta aplicação, caracterizando suas estruturas fundamentais em termos dos conceitos usualmente encontrados em Álgebra, como homomorfismos, radical, semisimplicidade, etc. Consideraremos também somente álgebras de dimensão finita.

Definição 1.4.1. Uma álgebra A sobre um corpo $F$ é chamada de álgebra bárica se admitir um homomorfismo de álgebras não trivial $w: A \rightarrow F$, chamado homo- 
morfismo peso. Denotamos por $(A, w)$. Para cada $x \in A, w(x)$ será chamado de peso de $x$.

Dado $x \in A$ tal que $w(x) \neq 0$, então temos a seguinte decomposição para $A$ :

$$
A=F x \oplus \operatorname{Ker}(w)
$$

De fato, sendo $w(x) \neq 0$, podemos escrever, para todo $a \in A$ :

$$
a=\frac{w(a)}{w(x)} x+\left(a-\frac{w(a)}{w(x)} x\right)
$$

A primeira parcela está, evidentemente, em Fx. Quanto à segunda, temos:

$$
w\left(a-\frac{w(a)}{w(x)} x\right)=w(a)-w\left(\frac{w(a)}{w(x)} x\right)=w(a)-\frac{w(a)}{w(x)} w(x)=w(a)-w(a)=0
$$

o que indica que $\left(a-\frac{w(a)}{w(x)} x\right) \in \operatorname{Ker}(w)$. A soma é direta, pois tomando $\alpha x \in F x$, se $\alpha x \in \operatorname{Ker}(w)$, então $w(\alpha x)=0, \log \alpha \alpha w(x)=0$, e como $w(x) \neq 0$, segue que $\alpha=0$, portanto $\alpha x=0$.

Consideraremos as álgebras báricas alternativas de dimensão finita sobre um corpo $F$ de característica $\neq 2$. Nosso principal objetivo nesta seção é mostrar que toda álgebra bárica alternativa possui um idempotente de peso 1. Vamos começar mostrando tal resultado para uma álgebra associativa. Para isto precisaremos dos três lemas seguintes.

Lema 1.4.1. Toda álgebra bárica associativa $(A, w)$ possui um idempotente.

Demonstração: Como $A$ é álgebra bárica, existe $x \in A$ tal que $w(x) \neq 0$. Então, $x$ não é nilpotente, pois se fosse teríamos:

$$
x^{n}=0 \Rightarrow w\left(x^{n}\right)=0 \Rightarrow(w(x))^{n}=0 \Rightarrow w(x)=0
$$

uma contradição. Como existe $x \in A$ tal que $x$ não é nilpotente, então $A$ é uma álgebra associativa que não é uma nilálgebra. Logo, pelo Lema 1.2.6, $A$ possui um idempotente.

Definição 1.4.2. Seja $(A, w)$ uma álgebra bárica. Chamamos de bar $(A)$ ao conjunto $\operatorname{bar}(A)=\{x \in A \mid w(x)=0\}$. 
Em vista da definição acima, se $B$ é uma subálgebra de $A$ e $B \nsubseteq \operatorname{bar}(A)$, dizemos que $B$ é uma subálgebra bárica de $A$. Neste caso dizemos que $\left(B,\left.w\right|_{B}\right)$ é uma álgebra bárica. Em outras palavras, uma subálgebra bárica é aquela que possui pelo menos um elemento de peso não nulo.

Lema 1.4.2. Se $(A, w)$ é uma álgebra bárica associativa $a \in A$, tal que $w(a)=1$, então Aa é uma subálgebra bárica de $A$.

Demonstração: Sempre existe em uma álgebra bárica um elemento $a$ de peso 1 . Como sabemos, existe $x \in A$ tal que $w(x) \neq 0$. Seja $a=\frac{x}{w(x)}$. Então:

$$
w(a)=w\left(\frac{x}{w(x)}\right)=w\left(\frac{1}{w(x)} x\right)=\frac{1}{w(x)} w(x)=1
$$

Mostremos que $A a$ é uma subálgebra de $A$. Tomando $b, c \in A$, temos $b a \in A a$ e $c a \in A a$. Daí, $(b a)(c a)=(b a c) a \in A a$. Logo, $A a$ é subálgebra. Para provar que $A a$ é uma subálgebra bárica de $A$, basta mostrar que existe $y \in A a$ tal que $w(y) \neq 0$. Tomando $y=a^{2} \in A a$, temos: $w\left(a^{2}\right)=w(a) w(a)=1$. Logo $y$ é um elemento de peso 1 em $A a$.

Lema 1.4.3. Se $(A, w)$ é uma álgebra bárica associativa $e$, para algum a $\in A$, com $w(a)=1$, tivermos $A a=A$, então $(A, w)$ possui um idempotente de peso 1.

Demonstração: Pelo Lema 1.2.6 podemos escrever: $A a=A$ se e somente se $x a \neq 0$, para todo $x \neq 0, x \in A$. Assim, $x a=0$ se e somente se $x=0$ (pois $a \neq 0$, uma vez que $w(a)=1$ ). Por hipótese, $A a=A$. Assim, $a \in A$ e também $a \in A a$, $\log a=e a$ para algum $e \in A$. Multiplicando ambos os membros por $e$ :

$$
e a=e^{2} a \Rightarrow\left(e-e^{2}\right) a=0 \Rightarrow e-e^{2}=0 \Rightarrow e=e^{2} .
$$

Provamos assim que é idempotente. Mas

$$
1=w(a)=w(e a)=w(e) w(a)=w(e) 1=w(e) \Rightarrow w(e)=1 .
$$

Teorema 1.4.4. Toda álgebra bárica associativa $(A, w)$ possui um idempotente de peso 1.

Demonstração: Seja $a \in A$ tal que $w(a)=1$. Faremos indução em $\operatorname{dim} A$. Se $\operatorname{dim} A=1$, então $A \cong F a$, onde $w(a)=1$. Pelo Lema 1.4.3, Fa possui um idempotente, isto é, $\exists \alpha \in F, \alpha \neq 0$ tal que $\alpha a$ é idempotente. Então 


$$
\begin{gathered}
(\alpha a)^{2}=\alpha a \Rightarrow \alpha^{2} a^{2}=\alpha a \Rightarrow \alpha a^{2}=a \Rightarrow w\left(\alpha a^{2}\right)=w(a)=1 \\
\Rightarrow \alpha w\left(a^{2}\right)=1 \Rightarrow \alpha w(a) w(a)=1 \Rightarrow \alpha \cdot 1.1=1 \Rightarrow \alpha=1 .
\end{gathered}
$$

Então $\alpha a=1 . a=a$ é um idempotente de peso 1. Suponhamos agora, por hipótese de indução, que o teorema é válido se $\operatorname{dim} A<n$. Seja $\operatorname{dim} A=n$. Consideraremos dois casos:

Caso 1: $A=A a$

Podemos aplicar o Lema 1.3.3, uma vez que $w(a)=1$. Assim, $A$ possui um idempotente de peso 1 .

Caso 2: $A \neq A a$

Neste caso, $\operatorname{dim} A a<\operatorname{dim} A, \operatorname{logo}$ pela hipótese de indução, $A a$ possui um idempotente de peso 1. Mas $A a \subseteq A$, logo, pelo Lema 1.4.2, $A a$ é uma subálgebra bárica de $A$. Portanto este mesmo idempotente de peso 1 de $A a$ é idempotente de peso 1 de $A$. Logo $A$ possui um idempotente de peso 1 .

Corolário 1.4.5. Se $(A, w)$ é uma álgebra bárica de potências associativas, então A possui um idempotente de peso 1.

Demonstração: Seja $x \in A$ tal que $w(x)=1$. A subálgebra $B=A(x)$ é bárica e associativa. $\operatorname{Logo} B$ possui um idempotente de peso 1 . Segue que $A$ possui um idempotente de peso 1.

Corolário 1.4.6. Se $(A, w)$ é uma álgebra bárica alternativa, então A possui um idempotente de peso 1.

Demonstração: Como $A$ é alternativa, então $A$ é de potências associativas (conseqüência do Teorema 1.2.4 (Artin)). Logo, pelo corolário anterior, $A$ possui um idempotente de peso 1.

A seguir apresentamos uma caracterização para álgebras báricas alternativas em termos de elementos do $\operatorname{bar}(A)$.

Proposição 1.4.7. Seja $(A, w)$ uma álgebra bárica com um idempotente e de peso 1. Então $(A, w)$ é alternativa se e somente se, para quaisquer $u, v \in \operatorname{bar}(A)$ são válidas as seguintes condições: 
(i) $(e, e, u)=(u, e, e)=(e, u, e)=0$;

(ii) $(e, u, u)=(u, u, e)=(u, e, u)=0$;

(iii) $(u, u, v)=(v, u, u)=0$;

(iv) $(e, u, v)+(u, e, v)=0$;

$(\mathrm{v})(v, e, u)+(v, u, e)=0$.

Demonstração: Se $A$ é alternativa, então as cinco condições acima são obviamente válidas. Precisamos provar apenas a recíproca. Seja então $(A, w)$ álgebra bárica na qual são válidas as igualdades indicadas. Tomamos $x=\alpha e+u \in A$ e $y=\beta e+v \in A$, com $\alpha, \beta \in F$. Temos:

$$
\begin{aligned}
(x, x, y) & =(\alpha e+u, \alpha e+u, \beta e+v) \\
& =(\alpha e, \alpha e, \beta e)+(\alpha e, \alpha e, v)+(\alpha e, u, \beta e)+(\alpha e, u, v) \\
& +(u, \alpha e, \beta e)+(u, \alpha e, v)+(u, u, \beta e)+(u, u, v) \\
& =\alpha^{2} \beta(e, e, e)+\alpha^{2}(e, e, v)+\alpha \beta(e, u, e)+\alpha(e, u, v) \\
& +\alpha \beta(u, e, e)+\alpha(u, e, v)+\beta(u, u, e)+(u, u, v)=0
\end{aligned}
$$

De maneira análoga provamos que $(y, x, x)=0$, resultando que $A$ é alternativa. 


\section{Capítulo 2}

\section{Radical e Semisimplicidade}

Neste capítulo trabalharemos com o importante conceito de radical, aplicado às álgebras alternativas e alternativas báricas. Trata-se de um conceito intimamente relacionado à estrutura de uma álgebra. Portanto iremos retomar tais idéias para refiná-las um pouco mais e estabelecer uma caracterização do radical nestes termos, visando em seguida estudar alguns resultados envolvendo a semisimplicidade das álgebras alternativas e báricas.

\section{$2.1 \quad$ O Radical}

Voltemos a considerar as álgebras nilpotentes. Lembrando, uma álgebra $A$ é chamada nilpotente se $A^{(n)}=0$, para algum $n \in \mathbb{N}, n>1$.

Vamos verificar que toda álgebra $A$ possui alguma subálgebra nilpotente. Em particular $A$ possui um ideal nilpotente. Mais ainda, veremos que em toda álgebra existe um ideal nilpotente que contém todos os outros ideais nilpotentes da referida álgebra.

Proposição 2.1.1. Toda álgebra $A$ de dimensão finita possui um único ideal nilpotente $R$ que contém todo ideal nilpotente $N$ de $A$.

Demonstração: Se o único ideal nilpotente de $A$ é 0 o teorema está provado. Seja então $R$ um ideal nilpotente não nulo tal que $\operatorname{dim} R$ seja máxima. Seja $N$ um ideal nilpotente não nulo, $N \neq R$. Claro que $R \cap N$ também é um ideal nilpotente. Calculemos:

$$
(R+N)^{m}=(R+N)^{m-1}(R+N), \text { onde }
$$


$m=\max \{r, n\}, r=$ índice de nilpotência de $R$ e $n=$ índice de nilpotência de $N$.

Provaremos que: $(R+N)^{m}=R^{m}+f(R, N)+N^{m}$, onde $f(X, Y)$ é um polinômio não associativo em $X$ e $Y$. Faremos indução em $m$.

A expressão é válida para $m=2$, pois:

$$
(R+N)^{2}=(R+N)(R+N)=R^{2}+R N+N R+N^{2} .
$$

Supondo agora válida para $m$, calculemos:

$$
\begin{aligned}
(R+N)^{m+1} & =(R+N)^{m}(R+N)=\left(R^{m}+f(R, N)+N^{m}\right)(R+N) \\
& =R^{m+1}+R^{m} N+f(R, N) R+f(R, N) N+N^{m} R+N^{m+1} \\
& =R^{m+1}+g(R, N)+N^{m+1}
\end{aligned}
$$

onde $g(R, N)=R^{m} N+f(R, N) R+f(R, N) N+N^{m} R$ é um polinômio nas condições desejadas.

Sendo $m \geq r$ e $m \geq n$, conforme indicado acima, resulta que $(R+N)^{m}=g(R, N)$, e portanto, $(R+N)^{m} \subseteq R \cap N$, logo $(R+N)^{m}$ é nilpotente à direita.

Mostremos que $R+N$ é nilpotente. De fato, se $(R+N)^{m}$ é nilpotente, existe $k \in \mathbb{N}$ tal que $\left((R+N)^{m}\right)^{k}=0$. Assim, para todo $x \in(R+N)$, temos que $x^{m} \in(R+N)^{m}$, $\operatorname{logo}\left(x^{m}\right)^{k}=0 \Rightarrow x^{m k}=0 \Rightarrow(R+N)$ é nilpotente à direita. Logo, pelo Corolário 1.2.10, $(R+N)$ é nilpotente de índice $m k$.

Mas $\operatorname{dim} R \geq \operatorname{dim}(R+N)$, devido à maximalidade da $\operatorname{dim} R$. Portanto $N \subseteq R$.

Definição 2.1.1. Seja A uma álgebra alternativa. Chama-se radical de A ao ideal nilpotente maximal de $A$. Notação: $R(A)$.

Convém notar que, embora o radical seja nilpotente e portanto uma nilálgebra, ou seja, todos os seus elementos são nilpotentes, o radical não é igual ao conjunto de todos os elementos nilpotentes. Vejamos, através de um exemplo simples, que pode haver elementos nilpotentes não nulos que não pertençam ao radical.

Contra-exemplo

Na álgebra das matrizes reais $M_{2}(\mathbb{R})$, temos que $a=\left[\begin{array}{ll}0 & 1 \\ 0 & 0\end{array}\right]$ é nilpotente de índice 2. Seja $z=\left[\begin{array}{ll}a & b \\ c & d\end{array}\right] \in M_{2}(\mathbb{R})$. Então $a z=\left[\begin{array}{ll}c & d \\ 0 & 0\end{array}\right]$ e $(a z)^{2}=\left[\begin{array}{ll}c^{2} & c d \\ 0 & 0\end{array}\right]$. $\operatorname{Assim}(a z)^{r} \neq 0$, se $c, d \neq 0$, para todo $r \in \mathbb{N}$. 
Pode-se obter uma caracterização do radical usando o conceito de nilpotência, considerando-se uma noção levemente modificada deste conceito, conforme definição a seguir.

Definição 2.1.2. Seja A uma álgebra alternativa. Um elemento $z \in A$ é chamado propriamente nilpotente se za é nilpotente, para todo $a \in A$.

Lema 2.1.2. Seja A álgebra alternativa. Um elemento $z \in A$ é propriamente nilpotente se e somente se az é nilpotente, para todo $a \in A$.

Demonstração: $(\Rightarrow) \quad z$ é propriamente nilpotente, então $z a$ é nilpotente, logo $(z a)^{m}=0$ para algum $m$. Pelo Teorema 1.2.4 (Artin), a subálgebra gerada por quaisquer dois elementos de uma álgebra alternativa $A$ é associativa. Então:

$$
(a z)^{m+1}=(a z)(a z) \ldots(a z)=a(z a) \ldots(z a) z=a(z a)^{m} z=a 0 z=0
$$

o que prova que $a z$ é nilpotente.

$(\Leftarrow)$ Analogamente, se az é nilpotente, temos:

$$
(z a)^{m+1}=(z a)(z a) \ldots(z a)=z(a z) \ldots(a z) a=z(a z)^{m} z=z 0 a=0
$$

provando que $z a$ é nilpotente. Portanto $z$ é propriamente nilpotente.

Lema 2.1.3. Seja A álgebra alternativa. Se $z \in A$ é propriamente nilpotente então $z$ é nilpotente.

Demonstração: Fazendo $a=z$, temos que: $z a=z z=z^{2}$ é nilpotente. Então:

$$
0=\left(z^{2}\right)^{r}=z^{2} \ldots z^{2}=z^{2 r}
$$

pois a subálgebra gerada por $z^{2}$ é de potências associativas (pois $A$ é alternativa e, toda álgebra alternativa é de potências associativas, como conseqüência do Teorema 1.2.4 (Artin)).

Designaremos por $\mathcal{B}$ o conjunto dos elementos propriamente nilpotentes de $A$.

Definição 2.1.3. Seja A uma álgebra. Um elemento $u \in A$ é chamado unidade de $A$ se $x u=u x=x, \forall x \in A$. Neste caso dizemos que A é uma álgebra com unidade. Denotamos a unidade de A por 1. 
Definição 2.1.4. Seja A uma álgebra com unidade. Dizemos que um elemento $x \in A$ tem inverso, denotado por $x^{-1}$, se $x x^{-1}=x^{-1} x=1$. Dizemos também que o elemento $x$ é invertível.

Lema 2.1.4. Seja A álgebra alternativa com unidade e $x \in A$ invertível. Então $\left(x, x^{-1}, y\right)=0$, para todo $y \in A$.

Demonstração: A seguinte identidade

$$
a(x, y, z)+(a, x, y) z=(a x, y, z)-(a, x y, z)+(a, x, y z)
$$

é trivialmente verificada. Basta apenas desenvolver os associadores. Fazendo as seguintes substituições: $a=x^{-1}, \quad y=x, z=x^{-1} y$, obtemos:

$$
\begin{aligned}
x^{-1} \overbrace{\left(x, x, x^{-1} y\right)}^{=0}+\overbrace{\left(x^{-1}, x, x\right)}^{=0} x^{-1} y & =\left(x^{-1} x, x, x^{-1} y\right)-\left(x^{-1}, x^{2}, x^{-1} y\right) \\
& +\left(x^{-1}, x, x\left(x^{-1} y\right)\right) \\
& \Rightarrow\left(x^{-1}, x^{2}, x^{-1} y\right)-\left(x^{-1}, x, x\left(x^{-1} y\right)\right)=0
\end{aligned}
$$

Desenvolvendo os associadores e aplicando a identidade (i) de Moufang (i) (Teorema 1.2.2), (xax)y=x(a(xy)), obtemos:

$$
\begin{gathered}
\left(x^{-1} x^{2}\right)\left(x^{-1} y\right)-x^{-1}\left(x^{2}\left(x^{-1} y\right)\right)+\left(x x^{-1}\right)\left(x\left(x^{-1} y\right)\right)-x\left(x^{-1}\left(x\left(x^{-1} y\right)\right)\right)=0 \\
\Rightarrow\left(x^{-1} x^{2}\right)\left(x^{-1} y\right)-\left(x^{-1} x^{2} x^{-1}\right) y+x\left(x^{-1} y\right)-x\left(\left(x^{-1} x x^{-1}\right) y\right)=0 \\
\Rightarrow\left(x^{-1} x^{2}\right)\left(x^{-1} y\right)-\left(x^{-1} x^{2} x^{-1}\right) y+x\left(x^{-1} y\right)-x\left(x^{-1} y\right)=0 \\
\Rightarrow\left(x^{-1} x^{2}\right)\left(x^{-1} y\right)-\left(x^{-1} x^{2} x^{-1}\right) y=0
\end{gathered}
$$

Note que: $x^{-1} x^{2}=x$. De fato:

$$
0=\left(x^{-1}, x, x\right)=\left(x^{-1} x\right) x-x^{-1} x^{2}=x-x^{-1} x^{2} \Rightarrow x^{-1} x^{2}=x
$$

A última igualdade obtida acima fica, portanto:

$$
x\left(x^{-1} y\right)-\left(x x^{-1}\right) y=0 \Rightarrow-\left(x, x^{-1}, y\right)=0
$$

Lema 2.1.5. O inverso, em uma álgebra alternativa $A$, é único.

Demonstração: Se $x^{\prime}$ é outro inverso de $x\left(x x^{\prime}=1\right)$, então 


$$
x^{\prime}=1 x^{\prime}=\left(x^{-1} x\right) x^{\prime}=x^{-1}\left(x x^{\prime}\right)=x^{-1} \cdot 1=x^{-1},
$$

usando que $\left(x, x^{-1}, x^{\prime}\right)=0$, pelo lema anterior.

Lema 2.1.6. Seja A álgebra alternativa de dimensão finita, com 1, sendo 1 o único idempotente de A. Então para todo $z \in A$, ou z tem inverso, $z^{-1} \in A$, ou z é propriamente nilpotente.

Demonstração: Seja $z \in A$ não nilpotente. $A(z)$ é uma subálgebra de $A$, associativa de dimensão finita que não é uma nilálgebra. Logo, segundo o Lema 1.3.1 (Albert), $A(z)$ contém um idempotente. Mas $A(z) \subset A$ e o único idempotente de $A$ é 1 . Logo

$$
\begin{gathered}
1=\alpha_{n} z^{n}+\alpha_{n-1} z^{n-1}+\cdots+\alpha_{1} z \quad \alpha_{i} \in F \\
\Rightarrow 1=z\left(\alpha_{n} z^{n-1}+\alpha_{n-1} z^{n-2}+\cdots+\alpha_{1}\right)
\end{gathered}
$$

Portanto $z$ é invertível.

Suponhamos agora $z$ nilpotente. Mostremos que $z$ é propriamente nilpotente. Se $z$ não for propriamente nilpotente, então existe $a \in A$ tal que $z a$ não é nilpotente. Logo, pela primeira parte deste lema, $z a$ tem inverso $(z a)^{-1}$.

Como $z$ é nilpotente, existe $m$ tal que $z^{m}=0$ e $z^{m-1} \neq 0$. Então:

$$
\begin{aligned}
0 \neq z^{m-1} & =z^{m-1}\left((z a)(z a)^{-1}\right) \stackrel{*}{=}\left(z^{m-1}(z a)\right)\left(z a^{-1}\right) \\
& =\left(\left(z^{m-1} z\right) a\right)\left(z a^{-1}\right)=\left(z^{m} a\right)\left(z a^{-1}\right)=0
\end{aligned}
$$

contradição. Portanto z é propriamente nilpotente.

(*) A subálgebra gerada por dois elementos de uma álgebra alternativa, é associativa.

Lema 2.1.7. Seja A álgebra alternativa de dimensão finita, com 1, sendo 1 o único idempotente de $A$. $O$ conjunto $\mathcal{B}$ dos elementos propriamente nilpotentes de $A$ é um ideal de A.

Demonstração: Para mostrar que $\mathcal{B}$ é um ideal de $A$ devemos mostrar três coisas:

(i) $z+z^{\prime} \in \mathcal{B}$, para todos $z, z^{\prime} \in \mathcal{B}$

Sejam $z, z^{\prime} \in \mathcal{B}$. Se $z+z^{\prime} \notin \mathcal{B}, z+z^{\prime}$ não é propriamente nilpotente, logo, pelo lema anterior, $z+z^{\prime}$ não é nilpotente. Então, ainda pelo lema anterior, $z+z^{\prime}$ tem um inverso $y$ e escrevemos:

$$
\left(z+z^{\prime}\right) y=1 \Rightarrow z y+z^{\prime} y=1 \quad \Rightarrow \quad z^{\prime} y=1-z y
$$


Mas $z y$ é propriamente nilpotente, pois $z \in \mathcal{B}$ e $y \in A$, logo $z y$ é nilpotente. Podemos escrever:

$$
\begin{gathered}
(z y)^{m}=0 \Rightarrow 1-(z y)^{m}=1 \\
=(1-z y)\left((z y)^{m-1}+(z y)^{m-2}+\cdots+(z y)^{2}+z y+1\right) \\
\Rightarrow(1-z y)^{-1}=1+z y+(z y)^{2}+\cdots
\end{gathered}
$$

Assim, $1-z y$ tem inverso. Mas $1-z y=z^{\prime} y$ e $z^{\prime} y$ é nilpotente, pois $z^{\prime} \in \mathcal{B}$ e $y \in A$. Mas $z^{\prime} y$ não pode ser ao mesmo tempo nilpotente e invertível. Chegamos a esta contradição, ao afirmarmos que $z+z^{\prime}$ não é propriamente nilpotente. Devemos ter, portanto, $z+z^{\prime}$ propriamente nilpotente, ou seja, $z+z^{\prime} \in \mathcal{B}$.

(ii) $\alpha z \in \mathcal{B}$, para todo $\alpha \in F$, e todo $z \in \mathcal{B}$

Sejam $\alpha \in F$ e $z \in \mathcal{B}$. Como $z$ é propriamente nilpotente, isto é, $z a$ é nilpotente, $\forall a \in A$, temos:

$$
\alpha(z a)=z(\alpha a)=(\alpha z) a
$$

Mas $z(\alpha a)$ é nilpotente, uma vez que $\alpha a \in A$. Daí, $(\alpha z) a$ é nilpotente. Portanto $\alpha z$ é propriamente nilpotente, isto é, $\alpha z \in \mathcal{B}$.

(iii) $a z \in \mathcal{B}$, para todo $a \in A$, e todo $z \in \mathcal{B}$

Sejam $a \in A, z \in \mathcal{B}$. Como $z$ é propriamente nilpotente, $a z$ é nilpotente, $\forall a \in$ $A$. Mas, nesta álgebra, como vimos no lema anterior, todo elemento nilpotente é também propriamente nilpotente. Então $a z$ é propriamente nilpotente, isto é, $a z \in \mathcal{B}$.

Definição 2.1.5. Em qualquer álgebra A, um idempotente e é chamado principal se não existir idempotente $u \in A$, não nulo, ortogonal a e.

A proposição a seguir dá uma caracterização para o idempotente principal em termos da subálgebra $A_{00}$ da decomposição de Peirce.

Proposição 2.1.8. Sejam A uma álgebra alternativa de dimensão finita e $e \in A$. Então e é um idempotente principal de $A$ se e somente se a subálgebra $A_{00}$ na decomposição de Peirce relativa a e, é uma nilálgebra. 
Demonstração: $(\Rightarrow)$ Seja $e \in A$ idempotente principal. Dado $x \in A_{00}$, então $x e=e x=0$. Logo $x$ é ortogonal a $e$. Então $x$ não é idempotente, pois se fosse, $e$ não seria principal. Então $A_{00}$ não tem idempotentes não nulos. Assim, de acordo com o Lema 1.3.3, $A_{00}$ é uma nilálgebra.

$(\Leftarrow)$ Seja $A_{00}$ uma nilálgebra. Então $\forall x \in A_{00}, x$ é nilpotente. Seja $u \in A$ um idempotente ortogonal a $e$. Então $u e=e u=0$, o que significa que $u \in A_{00}$, ou seja $u$ é nilpotente. Mas isto é uma contradição, pois se $u$ é nilpotente não pode ser idempotente. Então não podem existir idempotentes não nulos ortogonais a $e$. Logo $e$ é principal.

O próximo resultado garante a existência de idempotentes principais em qualquer álgebra alternativa de dimensão finita que não é uma nilálgebra.

Proposição 2.1.9. Qualquer álgebra alternativa $A$ de dimensão finita que não é uma nilálgebra contém um idempotente principal.

Demonstração: Pelo Lema 1.3.3, A contém um idempotente $e$. Se $e$ não é principal, existe um idempotente $u$ em $A$, ortogonal a $e$, isto é, $u^{2}=u, u \neq 0$ e $u e=e u=0$. Mas isto significa que $u \in A_{00}$. Além disso, $e^{\prime}=e+u$ é um idempotente e a subálgebra $A_{11, e^{\prime}}$ contém propriamente $A_{11}=A_{11, e}$. De fato, seja $x_{11} \in A_{11}$. Então:

$$
\begin{aligned}
& x_{11} e^{\prime}=x_{11} e+x_{11} u=x_{11} e=x_{11} \\
& e^{\prime} x_{11}=e x_{11}+u x_{11}=e x_{11}=x_{11}
\end{aligned}
$$

Logo, $x_{11} \in A_{11, e^{\prime}}$. E como $u \in A_{11, e^{\prime}}$ e $u \notin A_{11, e}$, segue que $\operatorname{dim} A_{11, e}<\operatorname{dim} A_{11, e^{\prime}}$. Se $e^{\prime}$ é principal, está demonstrado. Caso contrário repetimos o procedimento acima para um idempotente $u^{\prime} \in A$ ortogonal a $e^{\prime}$. O novo idempotente $e^{\prime \prime}=e^{\prime}+u^{\prime}$ deve gerar uma subálgebra $A_{11, e^{\prime \prime}}$ com dimensão maior que a dimensão de $A_{11, e^{\prime}}$. Como $A$ é de dimensão finita este processo deve acabar em algum momento, resultando então em um idempotente principal.

Os resultados a seguir permitirão conhecer com mais detalhes como são os elementos propriamente nilpotentes de uma álgebra $A$. A partir deste conhecimento ficará mais natural a relação destes elementos com o radical $R(A)$. 
Consideremos inicialmente a decomposição de Peirce de $A$ relativa a um idempotente principal $e$, a ser usada no próximo lema.

Lema 2.1.10. Seja A álgebra alternativa de dimensão finita e e $=e_{1}+\cdots+e_{t}$ um idempotente principal de $A$, onde os $e_{i}$ são idempotentes primitivos dois a dois ortogonais. Seja a decomposição de Peirce de $A$ em relação aos idempotentes $e_{1}, \ldots, e_{t}$. Então, para todo $i=1, \ldots, t$ :

(i) $e_{i}$ é a unidade de $A_{i i}$;

(ii) $e_{i}$ é o único idempotente de $A_{i i}$;

(iii) Se $x_{i i} \in A_{i i}$ é propriamente nilpotente em $A_{i i}$, então $x_{i i}$ é propriamente nilpotente em $A$.

Demonstração: Temos: $A_{i i}=\left\{x_{i i} \in A \mid e_{k} x_{i i}=\delta_{k i} x_{i i}, x_{i i} e_{k}=\delta_{i k} x_{i i}\right\}$. Se $k=i$, então $e_{i} x_{i i}=x_{i i} e_{i}=x_{i i}$, o que prova (i).

Se $u \in A_{i i}$ é um idempotente, então $e_{i}=u+\left(e_{i}-u\right)$. Mas $u\left(e_{i}-u\right)=u e_{i}-u^{2}=$ $u-u=0, \operatorname{logo} u$ e $\left(e_{i}-u\right)$ são ortogonais. Escrevemos então $e_{i}$ como soma de dois idempotentes ortogonais, o que contraria o fato de $e_{i}$ ser primitivo. Logo, $e_{i}$ é o único idempotente em $A_{i i}$, provando (ii).

Se $x_{i i}$ é propriamente nilpotente em $A_{i i}$, isto é $x_{i i} a_{i i}$ é nilpotente $\forall a_{i i} \in A_{i i}$, existe $r \in \mathbb{N}$ tal que $\left(x_{i i} a_{i i}\right)^{r}=0$

A Propriedade 11 da decomposição de Peirce estabelece que

$$
\left(x_{i i} a\right)^{m}=\left(x_{i i} a_{i i}\right)^{m-1} \sum_{k=0}^{t} x_{i i} a_{i k} .
$$

Fazendo $r=m-1$, temos que $\left(x_{i i} a\right)^{r+1}=0$. Assim $x_{i i} a$ é nilpotente $\forall a \in A$. Logo $x_{i i}$ é propriamente nilpotente em $A$ e, (iii) está provado.

Lema 2.1.11. Nas hipóteses do lema anterior, seja o conjunto

$$
G_{i j}=\left\{s_{i j} \in A_{i j} \mid \text { todos os elementos de } s_{i j} A_{j i} \text { são nilpotentes }\right\} .
$$

Cada $G_{i j}$ é um subespaço vetorial de $A$ e $G_{i j} \subseteq \mathcal{B}$, o conjunto dos elementos propriamente nilpotentes de A. 
Demonstração: Vamos mostrar primeiro que $G_{i j} \subseteq \mathcal{B}, i, j=0, \ldots, t$.

Pela Proposição 2.1.8, $A_{00}$ é uma nilálgebra. Assim, pela Propriedade 11 (Peirce), todo elemento em $A_{00}$ é propriamente nilpotente em $A$. Podemos concluir então que: $G_{00}=A_{00} \subseteq \mathcal{B}$.

É fácil ver que $G_{0 j}=A_{0 j}, j=1, \ldots, t$. Vamos mostrar agora que os elementos de $G_{0 j}$ são propriamente nilpotentes, o que significa que $G_{0 j} \subseteq \mathcal{B}$. Usando a Propriedade 12 (Peirce), fazendo $i=0$, temos:

$$
\left(x_{0 j} a\right)^{m}=\left(x_{0 j} a_{j 0}\right)^{m-1} \sum_{k=0}^{t} x_{0 j} a_{j k}+\left(x_{0 j} a_{0 j}\right)\left(x_{0 j} a_{j 0}\right)^{m-1}
$$

Mas $x_{0 j} a_{j 0} \in A_{00}$, logo é um elemento nilpotente. Fazendo $m-1=r(r=$ índice de nilpotência de $\left.x_{0 j} a_{j 0}\right)$, temos que: $\left(x_{0 j} a_{j 0}\right)^{r}=0$, e daí: $\left(x_{0 j} a\right)^{r+1}=0$. Isto mostra que $\left(x_{00} a\right)$ é nilpotente, ou seja $x_{0 j}$ é propriamente nilpotente. Portanto $G_{0 j} \subseteq \mathcal{B}$.

De maneira análoga provamos que $G_{i 0}=A_{i 0}, i=1, \ldots, t$. Para provar que $G_{i 0} \subseteq$ $\mathcal{B}$ usamos novamente a propriedade 12 da decomposição de Peirce, mas com uma leve adaptação, conforme indicamos a seguir. Fazendo $j=0$ na referida propriedade, vem:

$$
\left(x_{i 0} a\right)^{m}=\left(x_{i 0} a_{0 i}\right)^{m-1} \sum_{k=0}^{t} x_{i 0} a_{0 k}+\left(x_{i 0} a_{i 0}\right)\left(x_{i 0} a_{0 i}\right)^{m-1}
$$

O termo $\left(x_{i 0} a_{0 i}\right) \in A_{i i}$ não é necessariamente nilpotente. Aí fazemos:

$$
\begin{gathered}
\left(x_{i 0} a_{0 i}\right)^{m-1}=\left(x_{i 0} a_{0 i}\right)\left(x_{i 0} a_{0 i}\right) \ldots\left(x_{i 0} a_{0 i}\right)=x_{i 0}\left(a_{0 i} x_{i 0}\right) \ldots\left(a_{0 i} x_{i 0}\right) a_{0 i} \\
=x_{i 0}\left(a_{0 i} x_{i 0}\right)^{m-2} a_{0 i}
\end{gathered}
$$

Assim, o termo $\left(a_{0 i} x_{i 0}\right) \in A_{00}$, sendo portanto nilpotente. Daí $\left(x_{i 0} a\right)^{m}=0$ para algum $m$, o que significa que $x_{i 0}$ é propriamente nilpotente. Portanto $G_{i 0} \subseteq \mathcal{B}$.

Resta provar que $G_{i j} \subseteq \mathcal{B}$, para $i, j \neq 0$. Com o mesmo argumento utilizado para mostrar que $G_{i 0} \subseteq \mathcal{B}$, usando $j$ no lugar de 0 na propriedade 12 (Peirce), temos que se $x_{i j} \in G_{i j}$, então $\left(x_{i j} a_{j i}\right)$ é nilpotente. Logo, pela propriedade $12, x_{i j} a$ é nilpotente $\forall a \in A$. Mas isto quer dizer que $x_{i j}$ é propriamente nilpotente, ou seja $x_{i j} \in \mathcal{B}$. Portanto $G_{i j} \subseteq \mathcal{B}$.

Mostremos agora que cada $G_{i j}$ é um subespaço de $A$. Sejam $s_{i j}, s_{i j}^{\prime} \in G_{i j}$. Para que $G_{i j}$ seja subespaço de $A$, devemos ter:

(i) $\alpha s_{i j} \in G_{i j}$, para todo $\alpha \in F$; 
(ii) $s_{i j}+s_{i j}^{\prime} \in G_{i j}$.

Para provar (i) basta mostrar que $\alpha s_{i j} a_{j i}$ é nilpotente. Mas $s_{i j} a_{j i}$ é nilpotente, $\operatorname{logo},\left(s_{i j} a_{j i}\right)^{m}=0$, para algum $m$. Então:

$$
\left(\alpha s_{i j} a_{j i}\right)^{m}=\alpha^{m}\left(s_{i j} a_{j i}\right)^{m}=\alpha^{m} \cdot 0=0
$$

Quanto a (ii), devemos mostrar que $\left(s_{i j}+s_{i j}^{\prime}\right) a_{j i}$ é nilpotente, para todo $a_{j i} \in$ $A_{j i}$. Pelo Lema 2.1.10 $e_{i}$ é o único idempotente de $A_{i i}$. Agora, usando o Lema 2.1.7 o conjunto dos elementos propriamente nilpotentes de $A_{i i}$ é um ideal. Assim, $\left(s_{i j}+s^{\prime}+i j\right) a_{j i}=s_{i j} a_{j i}+s_{i j}^{\prime} a_{j i}$ é propriamente nilpotente em $A_{i i}$.

O próximo teorema estabelece uma relação importante entre o conjunto dos elementos propriamente nilpotentes e o radical.

Teorema 2.1.12 (Zorn). O radical $R$ de qualquer álgebra alternativa $A$ de dimensão finita é o conjunto $\mathcal{B}$ dos elementos propriamente nilpotentes de $A$.

Demonstração: Seja $G=\sum G_{i j}$. De acordo com o lema anterior cada $G_{i j} \subseteq \mathcal{B}$. Assim $G \subseteq\langle\mathcal{B}\rangle$. Mostremos que $G=\mathcal{B}$ e que $G$ é um ideal de $A$. Vamos dividir esta demonstração nos seguintes passos, onde mostraremos que:

(i) $\mathcal{B} \subseteq G$

Supondo por absurdo que $\mathcal{B}$ não está contido em $G$, existe $x \in \mathcal{B}$ tal que $x \notin G=\sum G_{i j}$. Mas $x=\sum x_{i j}$, e $x_{i j} \in A_{i j}$, com pelo menos um $x_{i j} \notin G_{i j}$. Mas, pelo lema anterior (2.1.11), $G_{i j}=A_{i j}$ se $i=0$ ou $j=0$. Logo $x_{i j} \notin G_{i j}$ requer necessariamente que $i, j \neq 0$.

Se $i=j, x_{i j} \in A_{j j}$, que é uma álgebra com 1, logo, pelo Lema 2.1.6, $x_{i j}$ tem inverso ou é propriamente nilpotente. Mas estamos supondo $x_{i j} \notin G_{i j} \subseteq \mathcal{B}$, $\operatorname{logo} x_{i j}$ não é propriamente nilpotente. Então $x_{i j}$ tem inverso $b_{j i}$ em $U_{j j}$ e podemos escrever

$$
b_{j i} x_{i j}=e_{j}
$$

Vamos mostrar que se $i \neq j$, temos a mesma equação para algum $b_{j i} \in A_{j i}$. Como $x_{i j} \notin G_{i j}$, existe $a_{j i} \in A_{j i}$ tal que $a_{j i} x_{i j} \in A_{j j}$ não é nilpotente. Logo $a_{j i} x_{i j}$ tem inverso $z_{j j} \in A_{j j}$ e temos:

$$
z_{j j}\left(a_{j i} x_{i j}\right)=e_{j}
$$


Mas, pela propriedade 6 (Peirce), $\left(z_{j j}, a_{j i}, x_{i j}\right)=0, \quad i \neq j$. Então posso associar, o que resulta:

$$
\left(z_{j j} a_{j i}\right) x_{i j}=e_{j}
$$

O elemento $z_{j j} a_{j i} \in A_{j i}$ é o inverso de $x_{i j}$. Assim, podemos considerar a mesma equação $b_{j i} x_{i j}=e_{j}$, tanto para $i=j$ como para $i \neq j$.

Pela propriedade 13 (Peirce):

$$
e_{i}\left(x_{i j} a\right)^{m}=\left(x_{i j} a_{j i}\right)^{m} \quad i, j=0,1, \ldots, t
$$

Fazendo as seguintes substituições: $i=j, \quad j=i, \quad x_{i j}=b_{j i}$ e $a=x$, temos:

$$
e_{j}\left(b_{j i} x\right)^{m} e_{j}=\left(b_{j i} x_{i j}\right)^{m}=e_{j}^{m}=e_{j} \neq 0
$$

Logo $b_{j i} x$ não é nilpotente, o que significa que $x$ não é propriamente nilpotente, isto é, $x \notin \mathcal{B}$, uma contradição.

Portanto, se $x \in \mathcal{B}$, devemos ter $x \in G$, e assim $\mathcal{B} \subseteq G$, como queríamos.

(ii) $G=\langle\mathcal{B}\rangle$

Como $G$ é subespaço de $A$, claro que $\langle\mathcal{B}\rangle \subseteq G$. E como, pelo Lema 2.1.11, $G \subseteq\langle\mathcal{B}\rangle$, segue que $G=\langle\mathcal{B}\rangle$

(iii) $G$ é um ideal de $A$

Mostraremos inicialmente as seguintes inclusões:

(iii.1) $G_{i j} A_{j k} \subseteq G_{i k} \quad i, j, k=0,1, \cdots, t$

(iii.2) $G_{i j} A_{i j} \subseteq G_{j i} \quad i \neq j, \quad i, j=1, \cdots, t$

Se $i=k$, seja $s_{i j} \in G_{i j}$. Então $s_{i j} A_{j i}$ é nilpotente e $s_{i j} A_{j i} \in A_{i i}$. Mas, notando que:

$$
G_{i i}=\left\{s_{i i} \in A_{i i} \mid \text { todos os elementos de } s_{i i} A_{i i} \text { são nilpotentes }\right\}
$$

temos que $s_{i j} A_{j i} \subseteq G_{i i}, \forall s_{i j} \in G_{i j}$. Então $G_{i j} A_{j i} \subseteq G_{i i}$. Se $i \neq k$, usamos a propriedade 6 (Peirce), fazendo:

$$
\left(G_{i j} A_{j k}\right) A_{k i}=G_{i j}\left(A_{j k} A_{k i}\right) \subseteq G_{i j} A_{j i} \subseteq G_{i i}
$$

Isto indica que $x_{i k} A_{k i}$ é nilpotente, onde $x_{i k} \in G_{i j} A_{j k}$. Mas, notando que: 


$$
G_{i k}=\left\{s_{i k} \in A_{i k} \mid \text { todos os elementos de } s_{i k} A_{k i} \text { são nilpotentes }\right\}
$$

temos que $x_{i k} A_{k i} \in G_{i k}$, para todo $x_{i k} \in G_{i k} A_{k i}$. Então: $G_{i j} A_{j k} \in G_{i k}$. Provamos, assim, a inclusão (iii.1).

Aplicando a propriedade 8 (Peirce), podemos escrever:

$$
\left(G_{i j} A_{i j}\right) A_{i j}=\left(A_{i j} A_{i j}\right) G_{i j} \subseteq A_{j i} G_{i j}
$$

Vamos mostrar que $A_{j i} G_{i j} \subseteq G_{j j}$.

Seja $s_{i j} \in G_{i j}$. Então $A_{j i} s_{i j}$ é nilpotente e $A_{j i} s_{i j} \subseteq A_{j j}$. Mas notando que:

$$
G_{j j}=\left\{s_{j j} \in A_{j j} \mid \text { todos os elementos de } A_{j j} s_{j j} \text { são nilpotentes }\right\}
$$

segue que $A_{j i} s_{i j} \subseteq G_{j j}$, para todo $s_{i j} \in G_{i j}$. Logo $A_{j i} G_{i j} \subseteq G_{j j}$. Assim, os elementos de $\left(G_{i j} A_{i j}\right) A_{i j}$ são nilpotentes, $\operatorname{logo} x_{j i} \in G_{i j} A_{i j} \quad \Rightarrow \quad x_{j i} \in G_{j i}$. Portanto $G_{i j} A_{i j} \subseteq G_{j i}$, concluindo a prova de (iii.2).

Podemos então escrever:

$$
G A=\left(\sum G_{i j}\right)\left(\sum A_{k l}\right)=\sum G_{i j} A_{j k}+\sum_{i \neq j} G_{i j} A_{i j} \subseteq G
$$

Esta última inclusão ocorre porque cada parcela está em $G$, de acordo com (iii.1) e (iii.2).

(iv) $\langle\mathcal{B}\rangle$ é nilpotente $(\log o\langle\mathcal{B}\rangle \subseteq R)$

Já mostramos que $G=\langle\mathcal{B}\rangle$ é um ideal de $A$. Provemos agora que $\langle\mathcal{B}\rangle$ é um ideal nilpotente. Supondo que $\langle\mathcal{B}\rangle$ não seja nilpotente, por estarmos em dimensão finita, $\langle\mathcal{B}\rangle$ não é nilálgebra. Então, pelo Lema 1.3.3, $\langle\mathcal{B}\rangle$ possui um idempotente $e=e_{1}+\cdots+e_{r}$.

$\operatorname{Mas}\langle\mathcal{B}\rangle=G=\sum G_{i j}, \operatorname{logo} e_{1} \in G_{11}$ é nilpotente, contradição. Então $\langle\mathcal{B}\rangle$ é nilpotente, $\log \langle\langle\mathcal{B}\rangle \subseteq R$. Mas $R \subseteq \mathcal{B}, \log$ o $\langle\mathcal{B}\rangle=\mathcal{B}$ e, portanto $R=\mathcal{B}$.

Corolário 2.1.13. Seja e um idempotente em uma álgebra alternativa $A$ de dimensão finita e $R$ radical de $A$. Seja a decomposição de Peirce de $A$ em relação a e, dada por $A=A_{11}+A_{10}+A_{01}+A_{00}$. Se $R_{i}$ é o radical de $A_{i i}$, então $R_{i}=R \cap A_{i i}, \quad(i=0,1)$. 
Demonstração: Seja $x_{i i} \in R_{i}$. Claro que $x_{i i} \in A_{i i}$ e $x_{i i}$ é propriamente nilpotente em $A_{i i}$. Então, pelo Lema 2.1.10, segue que $x_{i i}$ é propriamente nilpotente em $A$, logo $x_{i i} \in R$. Então $x_{i i} \in R \cap A_{i i}$ e portanto $R_{i} \subseteq R \cap A_{i i}$.

Reciprocamente, seja $x_{i i} \in R \cap A_{i i}$. Temos que $x_{i i}$ é propriamente nilpotente em $A$ (pois $x_{i i} \in R$ ). Mas como $x_{i i} \in A_{i i}, x_{i i}$ é propriamente nilpotente em $A_{i i}$, isto é, $x_{i i} \in R_{i}$. Logo $R \cap A_{i i} \subseteq R_{i}$. Daí, temos a igualdade desejada.

Corolário 2.1.14. Seja e um idempotente principal em uma álgebra alternativa $A$ de dimensão finita. Então $A_{10}+A_{01}+A_{00} \subseteq R$.

Demonstração: Pelo Lema 1.3.3 podemos escrever $e=e_{1}+\cdots+e_{t}$, com os $e_{i}$ idempotentes primitivos dois a dois ortogonais. Vamos provar que

$$
A_{10}=\sum_{i=1}^{t} G_{i 0}, \quad A_{01}=\sum_{j=1}^{t} G_{0 j}, \text { e } A_{00}=G_{00} .
$$

Esta última igualdade já foi provada no Lema 2.1.11, onde também foi provado que $G_{i 0}=A_{i 0}$ e $G_{0 j}=A_{0 j}, \quad i, j=1, \ldots, t$. Considerando a decomposição de Peirce de $A$ em relaçao aos $e_{i}$, temos, pela própria maneira como tal decomposição é definida, que $A_{10}=\sum_{i=1}^{t} A_{i 0}$, o que é equivalente a $A_{10}=\sum_{i=1}^{t} G_{i 0}$. Analogamente provamos que $A_{01}=\sum_{j=1}^{t} G_{0 j}$. Como, pelo mesmo lema os $G_{i j}$ são subespaços de $A$ e $G_{i j} \subseteq \mathcal{B}=R$, segue que $\sum_{i=1}^{t} G_{i 0}+\sum_{j=1}^{t} G_{0 j}+G_{00} \subseteq R$, ou $A_{10}+A_{01}+A_{00} \subseteq R$, conforme foi afirmado.

Definição 2.1.6. Uma álgebra alternativa $A$ é chamada semisimples se $R(A)=0$.

Teorema 2.1.15. Toda álgebra alternativa semisimples $A$ não nula, de dimensão finita possui unidade.

Demonstração: Como $A$ é semisimples, seu radical $R$ é nulo. Como $A \neq 0, A$ não é uma nilálgebra. De fato, se $A$ fosse nilálgebra, todo $x \in A$ seria nilpotente. Como $A$ é de dimensão finita, $A$ seria nilpotente. Mas $R$ é o ideal nilpotente maximal de $A, \operatorname{logo} A=R=0$, contradição.

Pelo Lema 1.3.3, se $A$ não é nilálgebra, $A$ possui um elemento idempotente. Nestas condições, $A$ possui um idempotente principal $e$, de acordo com a Proposição 2.1.9. 
Daí, pelo corolário anterior (2.1.14), considerando a decomposição de Peirce de $A$ relativa a $e$, temos:

$$
A_{10}+A_{01}+A_{00} \subseteq R=0 \Rightarrow A=A_{11}=e A e \Rightarrow e=1
$$

Definição 2.1.7. Uma álgebra A é chamada simples se A não é uma zero álgebra e os seus únicos ideais são 0 e A.

O teorema a seguir, devido a Zorn, estabelece a estrutura das álgebras semisimples, como soma direta de ideais simples. Sua demonstração será omitida, podendo ser encontrada em [22].

Teorema 2.1.16 (Zorn). Uma álgebra alternativa A não nula de dimensão finita é semisimples se e somente se $A=G_{1} \oplus \cdots \oplus G_{t}$, com $G_{i}$ ideais simples $(i=1, \ldots, t)$.

\section{Exemplos de álgebras semisimples}

1) Toda álgebra simples é semisimples.

É uma conseqüência direta do teorema anterior (2.1.16).

2) O produto cartesiano de uma família finita de álgebras simples de dimensão finita é uma álgebra semisimples.

Sejam $A_{1}, \ldots, A_{n}$ álgebras simples. Formamos o produto cartesiano

$A=A_{1} \times \cdots \times A_{n}=\left\{\left(a_{1}, \ldots, a_{n}\right) \mid a_{i} \in A_{i}, 1 \leq i \leq n\right\}$. Tal produto é uma álgebra se definirmos as operações componente a componente. Assim, se $a=\left(a_{1}, \ldots, a_{n}\right), b=\left(b_{1}, \ldots, b_{n}\right) \in A$, temos: $a+b=\left(a_{1}+b_{1}, \ldots, a_{n}+b_{n}\right) \quad \mathrm{e}$ $a b=\left(a_{1} b_{1}, \ldots, a_{n} b_{n}\right)$. Para $i=1, \ldots, n$, seja $I_{i}=0 \times \cdots \times 0 \times A_{i} \times 0 \times \cdots \times 0$. $I_{i}$ é um ideal simples de $A$, pois $A_{i}$ é simples. Como $A=I_{1} \oplus \cdots \oplus I_{n}$, então $A$ é semisimples.

3) $A / R(A)$ é semisimples.

A verificação de que o radical de $A / R(A)$ é nulo será feita no lema a seguir.

4) Seja $G$ um grupo finito e $F$ corpo. A álgebra de grupo $F G$ é definida por: $F G=\left\{\sum_{g \in G} \alpha_{g} g, \alpha_{g} \in F\right\} . F G$ é semisimples se e somente se $\operatorname{car} F=0$ ou $\operatorname{car} F$ não divide a ordem de $G$. Este é o Teorema de Maschke, cuja demonstração pode ser encontrada em [5]. 
Lema 2.1.17. Seja $(A, w)$ álgebra bárica alternativa. Então $R(A / R(A))=0$

Demonstração: $x \in R(A / R(A)) \Rightarrow x=a+R(A)$, com $a \in A$ e $x$ é propriamente nilpotente. Seja $n$ índice de nilpotência de $R(A)$. Então, dado $y \in A / R(A), y=$ $b+R(A)$. Daí: $0=(y x)^{n}=((b+R(A))(a+R(A)))^{n}=(b a)^{n}+(R(A))^{n}=(b a)^{n}+$ $R(A) \Rightarrow(b a)^{n} \in R(A)$. Assim, ba é nilpotente, o que significa que $a$ é propriamente nilpotente. Logo $a \in R(A)$ e $a+R(A)=x=0$.

\subsection{Decomposição de Peirce em álgebras báricas}

Vimos que uma álgebra bárica alternativa $(A, w)$ pode ser decomposta como $A=$ $F x \oplus \operatorname{Ker}(w)$, para algum $x \in A, \operatorname{com} w(x) \neq 0$.

Vimos também que $(A, w)$ (bárica alternativa) possui um idempotente de peso 1. Seja $e$ este idempotente. Podemos fazer a decomposição acima com $e$ no lugar de $x$ e ficamos com:

$$
A=F e \oplus \operatorname{Ker}(w) \quad \text { ou, equivalentemente } \quad A=F e \oplus \operatorname{bar}(A)
$$

Além disso, temos a decomposição de Peirce de $A$ em relação à $e$, dada por:

$$
\begin{gathered}
A=A_{11} \oplus A_{10} \oplus A_{01} \oplus A_{00}, \quad \text { onde } \\
A_{i j}=\left\{x_{i j} \in A \mid e x_{i j}=i x_{i j}, \quad x_{i j} e=j x_{i j}, \quad i, j=0,1\right\}
\end{gathered}
$$

A proposição seguinte relaciona estas duas decomposições.

Proposição 2.2.1. Tendo em vista as duas decomposições da álgebra bárica alternativa $(A, w)$, temos:

$$
A_{11}=F e \oplus \operatorname{bar}(A)_{11} \quad A_{10}=\operatorname{bar}(A)_{10} \quad A_{01}=\operatorname{bar}(A)_{01} \quad A_{00}=\operatorname{bar}(A)_{00}
$$

Demonstração: Explicitando a decomposição de $\operatorname{bar}(A)$ em termos de seus elementos, temos:

$$
\begin{aligned}
& \operatorname{bar}(A)_{11}=\{x \in \operatorname{bar}(A) \mid e x=x, x e=x\} \\
& \operatorname{bar}(A)_{10}=\{x \in \operatorname{bar}(A) \mid e x=x, x e=0\} \\
& \operatorname{bar}(A)_{01}=\{x \in \operatorname{bar}(A) \mid e x=0, x e=x\} \\
& \operatorname{bar}(A)_{00}=\{x \in \operatorname{bar}(A) \mid e x=0, x e=0\}
\end{aligned}
$$


Mostremos primeiro que $A_{11}=F e \oplus \operatorname{bar}(A)_{11}$. Seja $x \in F e \oplus \operatorname{bar}(A)_{11}$. Então $x=\alpha e+u, \alpha \in F, u \in \operatorname{bar}(A)_{11}$. Daí:

$$
\begin{aligned}
& x e=(\alpha e+u) e=(\alpha e) e+u e=\alpha e^{2}+u=\alpha e+u=x \\
& e x=e(\alpha e+u)=e \alpha e+e u=\alpha e^{2}+u=\alpha e+u=x
\end{aligned}
$$

Como $x e=x=e x$, segue que $F e \oplus \operatorname{bar}(A)_{11} \subseteq A_{11}$.

Seja agora $x \in A_{11}$. Considerando a decomposição original de $A$ como $A=$ $F e \oplus \operatorname{bar}(A)$, podemos escrever $x=\alpha e+u, \operatorname{com} \alpha \in F$, e $u \in \operatorname{bar}(A)$. Mas $x \in A_{11}$, então:

$$
\begin{aligned}
& x e=x \Rightarrow(\alpha e+u) e=\alpha e+u \Rightarrow \alpha e^{2}+u e=\alpha e+u \\
& \Rightarrow \alpha e+u e=\alpha e+u \Rightarrow u e=u \\
& e x=x \Rightarrow e(\alpha e+u)=\alpha e+u \Rightarrow e \alpha e+e u=\alpha e+u \\
& \Rightarrow \alpha e^{2}+e u=\alpha e+u \Rightarrow \alpha e+e u=\alpha e+u \Rightarrow e u=u
\end{aligned}
$$

Então, $u e=u=e u, \operatorname{logo} u \in \operatorname{bar}(A)_{11} \mathrm{e}, \operatorname{assim}, x \in F e \oplus \operatorname{bar}(A)_{11}, \operatorname{logo}$ $A_{11} \subseteq F e \oplus \operatorname{bar}(A)_{11}$. Com isto, provamos que $A_{11}=F e \oplus \operatorname{bar}(A)_{11}$.

Mostremos agora que $A_{10}=\operatorname{bar}(A)_{10}$. Evidentemente $\operatorname{bar}(A)_{10} \subseteq A_{10}$. Resta provar que $A_{10} \subseteq \operatorname{bar}(A)_{10}$. Seja $x \in A_{10}$. Então $e x=x$ e $x e=0$, e calculamos:

$$
w(x)=w(e x)=w(e) w(x)=w(x) w(e)=w(x e)=w(0)=0
$$

Daí, $x \in \operatorname{bar}(A)$. E como $e x=x$ e $x e=0$, segue que $x \in \operatorname{bar}(A)_{10}$. Logo $A_{10} \subseteq \operatorname{bar}(A)_{10}$, resultando então que $A_{10}=\operatorname{bar}(A)_{10}$.

De maneira inteiramente análoga se prova que $A_{01}=\operatorname{bar}(A)_{01}$

Mostremos finalmente que $A_{00}=\operatorname{bar}(A)_{00}$. É claro que $\operatorname{bar}(A)_{00} \subseteq A_{00}$. Basta mostrar que $A_{00} \subseteq \operatorname{bar}(A)_{00}$. Para tanto, seja $x \in A_{00}$. Então $x e=e x=0$. Afirmamos que $x \in \operatorname{bar}(A)$. De fato:

$$
w(x)=1 \cdot w(x)=w(e) \cdot w(x)=w(e x)=w(0)=0
$$

E como $x e=e x=0$, temos que $x \in \operatorname{bar}(A)_{00}, \log 0 A_{00} \subseteq \operatorname{bar}(A)_{00}$ e, portanto $A_{00}=\operatorname{bar}(A)_{00}$. 
Observações

- A decomposição de Peirce de $A$ em relação a $e$ fica:

$$
A=F e \oplus \operatorname{bar}(A)_{11} \oplus \operatorname{bar}(A)_{10} \oplus \operatorname{bar}(A)_{01} \oplus \operatorname{bar}(A)_{00}
$$

- Fe $\oplus \operatorname{bar}(A)_{11}$ é uma subálgebra bárica de $A$. De fato, se $x \in F e \oplus \operatorname{bar}(A)_{11}$, então existe $\alpha \in F$ e $u \in \operatorname{bar}(A)_{11}$ tal que

$$
x=\alpha e+u \text { e } w(x)=w(\alpha e+u)=\alpha w(e)+w(u)=\alpha \cdot 1+0=\alpha .
$$

E como $\alpha$ é arbitrário, o peso de $x$ não é zero. Assim, $F e \oplus \operatorname{bar}(A)_{11}$ é uma subálgebra bárica de $A$.

- $\operatorname{bar}(A)_{00}$ é uma subálgebra de $\operatorname{bar}(A)$ que não é bárica.

A proposição a seguir estabelece as condições para que um idempotente $e$ em uma álgebra bárica alternativa seja principal.

Proposição 2.2.2. Em uma álgebra bárica alternativa $(A, w)$, um elemento e é um idempotente principal se e somente se bar $(A)_{00}$ é uma nilálgebra.

Demonstração: $(\Rightarrow)$ Se e é um idempotente principal e $x \in \operatorname{bar}(A)_{00}$, então ex $=$ $x e=0$. Isto significa que $x$ é ortogonal a $e$. Logo $x$ não pode ser idempotente, pois, se fosse, e não seria principal. Então, $\operatorname{bar}(A)_{00}$ não possui idempotentes. Como $\operatorname{bar}(A)_{00}$ é subálgebra de $A$, segue que $\operatorname{bar}(A)_{00}$ é uma nilálgebra.

$(\Leftarrow)$ Se $\operatorname{bar}(A)_{00}$ é uma nilálgebra, $\operatorname{bar}(A)_{00}$ não possui idempotentes, pois todo elemento de $\operatorname{bar}(A)_{00}$ é nilpotente. Se $e$ não fosse principal, existiria um idempotente $x \in A$ ortogonal a $e$, isto é, $e x=x e=0, \log o x \in \operatorname{bar}(A)_{00}$, contradição. Portanto $e$ é principal.

Seja $e \in(A, w)$ um idempotente de peso 1. Sabemos que $e=e_{1}+\cdots+e_{n}$, com os $e_{i}$ idempotentes primitivos dois a dois ortogonais. Se é um idempotente, devemos ter $w(e)=0$ ou $w(e)=1$. De fato, se $w(e) \neq 0$, então $w(e)=w\left(e^{2}\right)=$ $w(e) w(e) \Rightarrow w(e)=1$. Assim, na decomposição acima, supondo $w(e)=1$, temos: $1=w(e)=w\left(e_{1}+\cdots+e_{n}\right)=w\left(e_{1}\right)+\cdots+w\left(e_{n}\right)$. Então, um dos $e_{i}$ tem peso 1 e os demais, peso 0. Vamos considerar: $w\left(e_{1}\right)=\ldots=w\left(e_{n-1}\right)=0$ e $w\left(e_{n}\right)=1$. Desta forma, escrevemos: 


$$
A=F e \oplus \operatorname{bar}(A) .
$$

Obtemos também uma decomposição mais refinada de $A$

$$
\begin{gathered}
A=\bigoplus_{i, j=1}^{n} A_{i j}, \quad \text { onde } \\
A_{i j}=\left\{x_{i j} \in A \mid e_{k} x_{i j}=\delta_{k i} x_{i j} \text { e } x_{i j} e_{k}=\delta_{j k} x_{i j}\right\} \\
i, j=0, \ldots, n ; \quad k=1, \ldots, n
\end{gathered}
$$

Proposição 2.2.3. Tendo em vista as duas decomposições da álgebra bárica alternativa $(A, w)$, temos:

$$
A_{n n}=F e_{n} \oplus \operatorname{bar}(A)_{n n}, \quad A_{i j}=\operatorname{bar}(A)_{i j}, \quad(i, j) \neq(n, n)
$$

Demonstração: É inteiramente análoga à Proposição 2.2.1

Com a notação da proposição anterior, escrevemos:

$$
A=F e_{n} \oplus\left(\bigoplus_{i, j=0}^{n} \operatorname{bar}(A)_{i j}\right)
$$

Consideremos agora a decomposição de Peirce de $\operatorname{bar}(A)$ em relação a

$$
u=e_{1}+\cdots+e_{n-1} .
$$

Para isto usaremos a notação $(\operatorname{bar}(A))_{i j}$ para diferenciar de $\operatorname{bar}(A)_{i j}$ (que se refere à decomposição em relação a $e$ ). Assim, temos:

$$
\operatorname{bar}(A)=\bigoplus_{i, j=0}^{n-1}(\operatorname{bar}(A))_{i j}
$$

A proposição a seguir relaciona as decomposições de $\operatorname{bar}(A)$ em relação a $e$ e a $u$.

Proposição 2.2.4. Na notação indicada acima, tem-se:

(i) $(\operatorname{bar}(A))_{00}=\operatorname{bar}(A)_{00} \oplus \operatorname{bar}(A)_{0 n} \oplus \operatorname{bar}(A)_{n 0} \oplus \operatorname{bar}(A)_{n n}$

(ii) $\bigoplus_{j=1}^{n-1}(\operatorname{bar}(A))_{0 j}=\bigoplus_{j=1}^{n-1}\left(\operatorname{bar}(A)_{0 j} \oplus \operatorname{bar}(A)_{n j}\right)$

(iii) $\bigoplus_{i=1}^{n-1}(\operatorname{bar}(A))_{i 0}=\bigoplus_{i=1}^{n-1}\left(\operatorname{bar}(A)_{i 0} \oplus \operatorname{bar}(A)_{i n}\right)$

(iv) $\bigoplus_{i, j=1}^{n-1}(\operatorname{bar}(A))_{i j}=\bigoplus_{i, j=1}^{n-1} \operatorname{bar}(A)_{i j}$ 


\section{Demonstração:}

(i) $(\operatorname{bar}(A))_{00}=\left\{x_{i j} \in \operatorname{bar}(A) \mid e_{k} x_{i j}=0, \quad x_{i j} e_{k}=0\right\}$

$(i, j=0, \ldots, n ; \quad k=1, \ldots, n-1)$

Como $e_{k} x_{i j}=\delta_{k i} x_{i j}$, para que um elemento $x_{i j}$ pertença a $(\operatorname{bar}(A))_{00}$, devemos ter $\delta_{k i}=0$, ou seja $k \neq i$. Portanto, $i=0$ e $i=n$.

Da mesma forma, sendo $x_{i j} e_{k}=\delta_{j k} x_{i j}$, para que $x_{i j} \in(\operatorname{bar}(A))_{00}$, devemos ter $\delta_{j k}=0$, para todos $j=1, \ldots, n$. Portanto $j=0$ e $j=n$.

Assim, os elementos de $(\operatorname{bar}(A))_{00}$ são todos da forma $x_{00}, x_{0 n}, x_{n 0}, x_{n n}$. Mas $x_{00} \in \operatorname{bar}(A)_{00}, x_{0 n} \in \operatorname{bar}(A)_{0 n}, x_{n 0} \in \operatorname{bar}(A)_{n 0}$ e $x_{n n} \in \operatorname{bar}(A)_{n n} . \log 0$

$$
(\operatorname{bar}(A))_{00} \subseteq \operatorname{bar}(A)_{00} \oplus \operatorname{bar}(A)_{0 n} \oplus \operatorname{bar}(A)_{n 0} \oplus \operatorname{bar}(A)_{n n}
$$

Mas como a inclusão contrária é claramente verdadeira, segue a igualdade.

(ii) $(\operatorname{bar}(A))_{0 j}=\left\{x_{i j} \in \operatorname{bar}(A) \mid e_{k} x_{i j}=0, \quad x_{i j} e_{k}=x_{i j}\right\}$

$(i, j=0, \ldots, n ; \quad k=1, \cdots, n-1)$

Para que $e_{k} x_{i j}=0$ devemos ter $k \neq i$, então $i=0$ e $i=n$.

Para que $x_{i j} e_{k}=x_{i j}$ devemos ter $k=j$, então $j=1, \ldots, n-1$. Portanto

$x_{i j} \in(\operatorname{bar}(A))_{0 j} \Rightarrow x_{i j}=x_{01}+\ldots+x_{0, n-1}+x_{n 1}+\ldots+x_{n, n-1}$

$\operatorname{Assim}(\operatorname{bar}(A))_{0 j} \subseteq \bigoplus_{j=1}^{n-1}\left(\operatorname{bar}(A)_{0 j}\right) \oplus \operatorname{bar}(A)_{n j}$

Como a outra inclusão é clara, segue a igualdade.

(iii) $(\operatorname{bar}(A))_{i 0}=\left\{x_{i j} \in \operatorname{bar}(A) \mid e_{k} x_{i j}=x_{i j}, \quad x_{i j} e_{k}=0\right\}$

$(i, j=0, \ldots, n ; \quad k=1, \cdots, n-1)$

Para que $e_{k} x_{i j}=x_{i j}$ devemos ter $k=i$, então $i=1, \ldots, n-1$.

Para que $x_{i j} e_{k}=0$ devemos ter $k \neq j$, então $j=1$ e $j=n$. Portanto

$x_{i j} \in(\operatorname{bar}(A))_{i 0} \Rightarrow x_{i j}=x_{10}+\ldots+x_{n-1,0}+x_{1 n}+\ldots+x_{n-1, n}$

$\operatorname{Assim}(\operatorname{bar}(A))_{i 0} \subseteq \bigoplus_{i=1}^{n-1}\left(\operatorname{bar}(A)_{i 0} \oplus \operatorname{bar}(A)_{i n}\right.$

Também a outra inclusão é imediata, e temos então a igualdade. 
(iv) $(\operatorname{bar}(A))_{i j}=\left\{x_{i j} \in \operatorname{bar}(A) \mid e_{k} x_{i j}=x_{i j}, \quad x_{i j} e_{k}=x_{i j}\right\}$

$i, j=0, \ldots, n ; \quad k=1, \cdots, n-1$

Para que $e_{k} x_{i j}=x_{i j}$ devemos ter $k=i$, então $i=1, \ldots, n-1$.

Para que $x_{i j} e_{k}=x_{i j}$ devemos ter $k=j$, então $j=1, \ldots, n-1$.

Os elementos de $(\operatorname{bar}(A))_{i j}(i, j) \neq(n, n)$ devem ser da forma:

$x_{i j}=x_{11}+\cdots+x_{1 n}+\cdots+x_{n-1,1}+\cdots+x_{n-1, n-1}$

Analogamente aos casos anteriores, obtemos:

$$
(\operatorname{bar}(A))_{i j}=\bigoplus_{i, j=1}^{n-1} \operatorname{bar}(A)_{i j}
$$

Proposição 2.2.5. Sejam $(A, w)$ álgebra bárica alternativa, e idempotente principal em $(A, w)$, com $e=e_{1}+\cdots+e_{n}, w(e)=w\left(e_{n}\right)=1, e_{i}$ idempotentes primitivos dois a dois ortogonais. Então $u=e_{1}+\cdots+e_{n-1}$ é um idempotente principal em bar $(A)$.

Demonstração: Considerando a decomposição de Peirce de $\operatorname{bar}(A)$ em relação a $u$, temos, na notação da proposição anterior:

$$
\operatorname{bar}(A)=(\operatorname{bar}(A))_{00} \oplus\left(\bigoplus _ { j = 1 } ^ { n - 1 } ( \operatorname { b a r } ( A ) ) _ { 0 j } \oplus \left(\bigoplus_{i=1}^{n-1}(\operatorname{bar}(A))_{i 0} \oplus\left(\bigoplus_{i, j=1}^{n-1}(\operatorname{bar}(A))_{i j}\right)\right.\right.
$$

Para que $u$ seja principal, $(\operatorname{bar}(A))_{00}$ deve ser uma nilálgebra (de acordo com a Proposição 2.2.2). Sabemos, da proposição anterior (2.2.4), que:

$$
(\operatorname{bar}(A))_{00}=\operatorname{bar}(A)_{00} \oplus \operatorname{bar}(A)_{0 n} \oplus \operatorname{bar}(A)_{n 0} \oplus \operatorname{bar}(A)_{n n}
$$

Seja $x \in(\operatorname{bar}(A))_{00}$. Podemos escrever $x=b+x_{n n}$, com

$$
b \in \operatorname{bar}(A)_{00} \oplus \operatorname{bar}(A)_{0 n} \oplus \operatorname{bar}(A)_{n 0} \subseteq R(A) \text { e } x_{n n} \in \operatorname{bar}(A)_{n n} .
$$

Como $A_{n n}=F e_{n} \oplus \operatorname{bar}(A)_{n n}$, pelo Lema $2.1 .10 e_{n}$ é o único idempotente em $A_{n n}, \operatorname{logo} \operatorname{bar}(A)_{n n}$ não possui idempotentes. Então, a Proposição 2.1.9 garante que $\operatorname{bar}(A)_{n n}$ é uma nilálgebra. Assim, $x_{n n}$ é nilpotente. Sendo $e_{n}$ o único idempotente em $A_{n n}$, estamos nas condições do Lema 2.1.6, em cuja demonstração ficou provado que todo elemento nilpotente em $A_{n n}$ é também propriamente nilpotente. Temos então, $x_{n n}$ propriamente nilpotente, $\operatorname{logo} x_{n n} \in R(A)$.

Assim $x=b+x_{n n} \in R(A)$ (pois $R(A)$ é ideal de $A, b \in R(A), x_{n n} \in R(A)$ ), $\log 0$ $x$ é propriamente nilpotente, o que significa (Lema 2.1.3) que $x$ é nilpotente. Sendo 
$x \in(\operatorname{bar}(A))_{00}$ arbitrário, segue que $(\operatorname{bar}(A))_{00}$ é uma nilálgebra. Portanto $u$ é um idempotente principal em $\operatorname{bar}(A)$.

Proposição 2.2.6. Seja $(A, w)$ uma álgebra bárica alternativa e e $\in A$ um idempotente de peso 1. Então bar $(A)$ é uma nilálgebra se e somente se e é um idempotente primitivo e principal.

Demonstração: $(\Rightarrow)$ Se $e$ não é primitivo, então $e=u+v$, com $u$ e $v$ idempotentes ortogonais não nulos, isto é, $u v=v u=0, u^{2}=u, v^{2}=v$ Como $w(e)=1$, então se $w(u)=1$, deveríamos ter $v \in \operatorname{bar}(A)$, contradição, pois $\operatorname{bar}(A)$ é uma nilálgebra. Então $e$ tem que ser primitivo. Se $e$ não é principal, então existe $u \in A$ tal que $e u=u e=0$ e $u^{2}=u$. $\operatorname{logo} u \in \operatorname{bar}(A)$, pois $0=w(e u)=w(e) w(u)=w(u)$. Contradição. Logo $e$ deve ser principal.

$(\Leftarrow)$ Seja a decomposição de $A$ em relação a $e$, dada por

$$
A=F e \oplus \operatorname{bar}(A)_{11} \oplus \operatorname{bar}(A)_{10} \oplus \operatorname{bar}(A)_{01} \oplus \operatorname{bar}(A)_{00}
$$

que também pode ser escrita: $A=F e \oplus \operatorname{bar}(A)$.

Pela Proposição 2.2.5 $\operatorname{bar}(A)_{11}$ é uma nilálgebra e, além disso, e é o único idempotente de $A_{11}=F e \oplus \operatorname{bar}(A)_{11}$. Assim, o Lema 2.1.6 pode ser aplicado, e todo elemento nilpotente de $\operatorname{bar}(A)_{n n}$ é também propriamente nilpotente. Daí bar $(A)_{n n} \subseteq R(A)$. Mas, sabemos que $\operatorname{bar}(A)_{10} \oplus \operatorname{bar}(A)_{01} \oplus \operatorname{bar}(A)_{00} \subseteq R(A)$, (Corolário 2.1.14) logo $\operatorname{bar}(A) \subseteq R(A)$. Portanto $\operatorname{bar}(A)$ é nilpotente e, consequentemente, bar $(A)$ é uma nilálgebra.

\subsection{O radical e o bar radical}

Nesta seção vamos definir o bar radical de uma álgebra bárica. Começaremos com uma proposição que relaciona o radical de $A$ com o radical de $\operatorname{bar}(A)$.

Proposição 2.3.1. Sejam $(A, w)$ álgebra bárica alternativa, $R(A)$ o radical de $A e$ $R(\operatorname{bar}(A))$ o radical de bar $(A)$. Então $R(A)=R(\operatorname{bar}(A))$.

Demonstração: Como $R(A)$ é uma nilálgebra, então $R(A) \subset \operatorname{bar}(A)$.

$$
\left(x \in R(A) \Rightarrow x^{n}=0 \Rightarrow w\left(x^{n}\right)=(w(x))^{n}=0 \Rightarrow w(x)=0\right)
$$


Sendo $R(A)$ um ideal de $A$, e $R(A) \subset \operatorname{bar}(A) \subseteq A$, temos que $R(A)$ também é um ideal (nilpotente) de $\operatorname{bar}(A)$. Logo $R(A) \subseteq R(\operatorname{bar}(A))$. Resta mostrar que $R(\operatorname{bar}(A)) \subseteq R(A)$. Para isto, é suficiente mostrar que $R(\operatorname{bar}(A))$ é um ideal de $A$.

Considerando a decomposição de Peirce de $\operatorname{bar}(A)$ em relação a $u=e_{1}+\cdots+e_{n-1}$ dada na Proposição 2.2.5:

$$
\operatorname{bar}(A)=(\operatorname{bar}(A))_{00} \oplus\left(\bigoplus _ { j = 1 } ^ { n - 1 } ( \operatorname { b a r } ( A ) ) _ { 0 j } \oplus \left(\bigoplus_{i=1}^{n-1}(\operatorname{bar}(A))_{i 0} \oplus\left(\bigoplus_{i, j=1}^{n-1}(\operatorname{bar}(A))_{i j}\right)\right.\right.
$$

Temos, pelo Corolário 2.1.14, que:

$$
(\operatorname{bar}(A))_{00} \oplus\left(\bigoplus _ { j = 1 } ^ { n - 1 } ( \operatorname { b a r } ( A ) ) _ { 0 j } \oplus \left(\bigoplus_{i=1}^{n-1}(\operatorname{bar}(A))_{i 0} \subseteq R(\operatorname{bar}(A))\right.\right.
$$

Agora, usando a decomposição de Peirce de $A$ em relação ao idempotente principal $e$, temos que, de acordo com a Proposição 2.2.4, um elemento $b \in R(\operatorname{bar}(A))$ pode ser escrito de maneira única como:

$$
b=\left(b_{00}+b_{0 n}+b_{n 0}+b_{n n}\right)+\sum_{j=1}^{n-1}\left(b_{0 j}+b_{n j}\right)+\sum_{i=1}^{n-1}\left(b_{i 0}+b_{i n}\right)+\sum_{i, j=1}^{n-1} b_{i j}
$$

Para mostrar que $R(\operatorname{bar}(A))$ é um ideal de $A$, precisamos mostrar que $e_{n} b \in A$ e que $b e_{n} \in A$, pois $w\left(e_{n}\right)=1$ e $w\left(e_{i}\right)=0$ para todo $i=1, \ldots, n-1$.

$$
\begin{aligned}
e_{n} b= & e_{n} b_{00}+e_{n} b_{0 n}+e_{n} b_{n 0}+e_{n} b_{n n}+\sum_{j=1}^{n-1}\left(e_{n} b_{0 j}+e_{n} b_{n j}\right) \\
& +\sum_{i=1}^{n-1}\left(e_{n} b_{i 0}+e_{n} b_{i n}\right)+\sum_{i, j=1}^{n-1} e_{n} b_{i j} \\
\Rightarrow e_{n} b=b_{n 0} & +b_{n n}+\sum_{j=1}^{n-1} b_{n j} \in(\operatorname{bar}(A))_{00} \oplus\left(\bigoplus_{j=1}^{n-1}(\operatorname{bar}(A))_{0 j}\right) \subseteq R(\operatorname{bar}(A)) \\
b e_{n}= & b_{00} e_{n}+b_{0 n} e_{n}+b_{n 0} e_{n}+b_{n n} e_{n}+\sum_{j=1}^{n-1}\left(b_{0 j} e_{n}+b_{n j} e_{n}\right) \\
& +\sum_{i=1}^{n-1}\left(b_{i 0} e_{n}+b_{i n} e_{n}\right)+\sum_{i, j=1}^{n-1} b_{i j} e_{n} \\
\Rightarrow b e_{n}=b_{0 n} & +b_{n n}+\sum_{i=1}^{n-1} b_{i n} \in(\operatorname{bar}(A))_{00} \oplus\left(\bigoplus_{i=1}^{n-1}(\operatorname{bar}(A))_{i 0}\right) \subseteq R(\operatorname{bar}(A))
\end{aligned}
$$


Provamos assim, que $R(\operatorname{bar}(A))$ é um ideal de $A$, logo $R(\operatorname{bar}(A)) \subseteq R(A)$. Das duas inclusões resulta que $R(\operatorname{bar}(A))=R(A)$, como queríamos.

Para avançarmos em nosso objetivo precisaremos estudar algumas propriedades válidas para as álgebras báricas arbitrárias (não necessariamente alternativas). Tais propriedades permitirão caracterizar o conceito essencial desta seção, o bar radical, que definiremos em breve.

Definição 2.3.1. Sejam $(A, w),(B, \gamma)$ álgebras báricas e $\varphi: A \rightarrow B$ homomorfismo de álgebras tal que $\gamma \circ \varphi=w$.

(i) $\varphi$ é chamado homomorfismo bárico.

(ii) Se $\varphi$ for sobrejetor será chamado epimorfismo bárico.

(iii) Se $\varphi$ for bijetor dizemos que $\varphi$ é um isomorfismo bárico. Neste caso, as álgebras báricas $(A, w)$ e $(B, \gamma)$ são ditas isomorfas e denotamos $(A, w) \cong_{b}$ $(B, \gamma)$.

Definição 2.3.2. Um ideal I de uma álgebra bárica alternativa $(A, w)$ é chamado ideal bárico de $A$ se $I \subseteq \operatorname{bar}(A)$

Definição 2.3.3. Sejam $(A, w)$ uma álgebra bárica e $B$ subálgebra de $A$ tal que $\operatorname{bar}(B)$ é um ideal bilateral de A. Então B é chamada subálgebra bárica normal. Notação: $B \unlhd A$ ou $B \triangleleft A$, se $B \neq A$.

Lema 2.3.2. Sejam $(A, w),(B, \gamma)$ álgebras báricas e $\varphi: A \rightarrow B$ um homomorfismo bárico. Então tem-se:

(i) $\operatorname{ker} \varphi$ é um ideal bárico de $A$;

(ii) $S e C \leq A$, então $\varphi(C) \leq B$ e bar $(\varphi(C))=\varphi(\operatorname{bar}(C))$;

(iii) $S e C \unlhd D \leq A$, então $\varphi(C) \unlhd \varphi(D) \leq B$.

\section{Demonstração:}

(i) Basta mostrar que ker $\varphi \subseteq \operatorname{bar}(A)$. Seja $x \in \operatorname{ker} \varphi$. Então $\varphi(x)=0$. Mas $w=\gamma \circ \varphi, \log \circ w(x)=(\gamma \circ \varphi)(x)=\gamma(\varphi(x))=\gamma(0)=0 \Rightarrow x \in \operatorname{bar}(A)$. Portanto ker $\varphi \subseteq \operatorname{bar}(A)$. 
(ii) É imediato que $\varphi(C) \leq B$. Mostremos que $\operatorname{bar}(\varphi(C))=\varphi(\operatorname{bar}(C))$. Seja $x \in \operatorname{bar}(\varphi(C))$. Então, como $\varphi(C)$ é subálgebra de $B$, temos $\gamma(x)=0$, e ainda $x \in \varphi(C)$, donde $x=\varphi(c)$ para algum $c \in C$. Assim

$0=\gamma(x)=\gamma(\varphi(c))=(\gamma \circ \varphi)(x)=w(c)$. Então

$c \in \operatorname{bar}(A)$ e $x=\varphi(c) \in \varphi(\operatorname{bar}(C))$. Logo $\operatorname{bar}(\varphi(C)) \subseteq \varphi(\operatorname{bar}(C))$.

Seja agora $x \in \varphi(\operatorname{bar}(C))$. Existe $c \in \operatorname{bar}(C)$ tal que $x=\varphi(c)$.

Daí, $x \in \varphi(C)$ e $\gamma(x)=\gamma(\varphi(c))=(\gamma \circ \varphi)(x)=w(c)=0 \Rightarrow x \in \operatorname{bar}(\varphi(C))$.

$\operatorname{Logo} \varphi(\operatorname{bar}(C)) \subseteq \operatorname{bar}(\varphi(C))$.

(iii) Basta provar que $\operatorname{bar}(\varphi(C))$ é um ideal bilateral de $\operatorname{bar}(\varphi(D))$. Sejam $x \in$ $\operatorname{bar}(\varphi(D))$ e $y \in \operatorname{bar}(\varphi(C))$. Então, por (ii), $x \in \varphi(\operatorname{bar}(D))$ e $y \in \varphi(\operatorname{bar}(C))$, e podemos escrever $x=\varphi(D)$, com $d \in \operatorname{bar}(D)$ e $y=\varphi(C)$, com $c \in \operatorname{bar}(C)$. Daí, $x y=\varphi(d) \varphi(c)=\varphi(d c)$. Mas como $C \unlhd D, \operatorname{bar}(C)$ é um ideal bilateral de $\operatorname{bar}(D)$, então $d c \in \operatorname{bar}(C)$, logo $x y=\varphi(d c) \in \varphi(\operatorname{bar}(C))=\operatorname{bar}(\varphi(C))$. Portanto $\operatorname{bar}(\varphi(C))$ é ideal bilateral de $\operatorname{bar}(\varphi(D))$.

Definição 2.3.4. Sejam $(A, w)$ uma álgebra bárica e $B \subseteq A$.

(i) B é uma subálgebra bárica maximal (subálgebra bárica maximal normal) de $(A, w)$ se $B<A(B \triangleleft A)$ e se $C \leq A(C \unlhd A)$ tal que $B \subseteq C$, então $C=B$ ou $C=A$.

(ii) B é um ideal bárico maximal de $(A, w)$ se $B$ é um ideal bárico de $(A, w), B \neq$ $\operatorname{bar}(A)$ e se $J$ é um ideal bárico de $(A, w)$ tal que $B \subseteq J$, então $J=B$ ou $J=\operatorname{bar}(A)$.

Lema 2.3.3. Sejam $(A, w)$ e $(B, \gamma)$ álgebras báricas e $\varphi: A \rightarrow B$ um epimorfismo bárico. Então as seguintes condições são válidas:

(i) $S e C \leq B$, então $\varphi^{-1}(C) \leq A$ e bar $\left(\varphi^{-1}(C)\right)=\varphi^{-1}(\operatorname{bar}(C))$;

(ii) $S e C \unlhd D \leq B$, então $\varphi^{-1}(C) \unlhd \varphi^{-1}(D) \leq A$;

(iii) $S e C \leq A(C \unlhd A)$ é maximal e ker $\varphi \subseteq C$, então $\varphi(C) \leq B(\varphi(C) \unlhd B)$ é maximal;

(iv) $S e C<B(C \triangleleft B)$ é maximal, então $\varphi^{-1}(C)<A\left(\varphi^{-1}(C) \triangleleft A\right)$ é maximal. 


\section{Demonstração:}

(i) É fácil ver que $\varphi^{-1}(C)$ é subálgebra de $A$. Agora, seja $x \in \operatorname{bar}\left(\varphi^{-1}(C)\right)$. Temos que $x \in \varphi^{-1}(C)$, $\operatorname{logo} \varphi(x) \in C$. Além disso, $w(x)=0$. Escrevemos então: $0=w(x)=(\gamma \circ \varphi)(x)=\gamma(\varphi(x)) \Rightarrow \varphi(x) \in \operatorname{bar}(C)$ e $x \in \varphi^{-1}(\operatorname{bar}(C))$. Logo $\operatorname{bar}\left(\varphi^{-1}(C)\right) \subseteq \varphi^{-1}(\operatorname{bar}(C))$. Seja agora $x \in \varphi^{-1}(\operatorname{bar}(C))$. Então existe $c \in \operatorname{bar}(C)$ tal que $\varphi(x)=c$. Mas se $c \in \operatorname{bar}(C)$, então $\gamma(c)=0$ e escrevemos: $0=\gamma(c)=\gamma(\varphi(x))=(\gamma \circ \varphi)(x)=w(x)$. Assim, $x \in \operatorname{bar}\left(\varphi^{-1}(\operatorname{bar}(C))\right)$. Logo, pela primeira inclusão, $x \in \varphi^{-1}(\operatorname{bar}(\operatorname{bar}(C)))=\varphi^{-1}(\operatorname{bar}(C))$.

(ii) Devemos mostrar que $\operatorname{bar}\left(\varphi^{-1}(C)\right)$ é ideal bilateral de $\operatorname{bar}\left(\varphi^{-1}(D)\right)$. Tomemos $a \in \operatorname{bar}\left(\varphi^{-1}(C)\right)$ e $b \in \operatorname{bar}\left(\varphi^{-1}(D)\right)$. Então, por (i), $a \in \varphi^{-1}(\operatorname{bar}(C))$ e $b \in$ $\varphi^{-1}(\operatorname{bar}(D))$, donde $\varphi(a) \in \operatorname{bar}(C), \varphi(b) \in \operatorname{bar}(D) \Rightarrow \varphi(a) \varphi(b)=\varphi(a b) \in$ $\operatorname{bar}(D)$, pois $C \unlhd D$. Logo $a b \in \varphi^{-1}(\operatorname{bar}(D)) \stackrel{(i)}{=} \operatorname{bar}\left(\varphi^{-1}(D)\right)$. De maneira análoga chegamos a $b a \in \operatorname{bar}\left(\varphi^{-1}(D)\right)$.

(iii) Por 2.3.2 (ii) temos que $\varphi(C) \leq B$. Devemos mostrar que $\varphi(C)<B$, isto é, $\varphi(C) \neq B$. Supondo que $\varphi(C)=B$, teremos $C=\varphi^{-1}(\varphi(C))=\varphi^{-1}(B)=A$, contradição, pois $C<A$. Para mostrar que $\varphi(C)$ é maximal, seja $D \leq A$ tal que $\varphi(C) \subseteq D$. Então, por (ii), $\varphi^{-1}(\varphi(C)) \leq \varphi^{-1}(D) \leq A$ ou $C \leq \varphi^{-1}(D) \leq A$. Mas como $C$ é maximal, devemos ter $\varphi^{-1}(D)=C$ ou $\varphi^{-1}(D)=A$. Daí, $D=\varphi(C)$ ou $D=\varphi(A)=B$. Portanto $\varphi(C)$ é maximal.

$\mathrm{O}$ caso $C \unlhd A \Rightarrow \varphi(C) \unlhd B$ é maximal, se prova de modo análogo.

(iv) Por 2.3.2 (ii) temos que $\varphi(C) \leq A$. Se $\varphi^{-1}(C)=A$, então $C=\varphi\left(\varphi^{-1}(C)\right)=$ $\varphi(A)=B$, contradição, pois $C<B$. Seja agora $D \leq A$ tal que $\varphi^{-1}(C) \subseteq D$. Então $C \subseteq \varphi(D)$ e por 2.3.2 (ii), $\varphi(D) \leq B$. Daí, $C \subseteq \varphi(D) \leq B$, mas sendo $C$ maximal, segue que $\varphi(D)=C$ ou $\varphi(D)=B$. Logo, $D=\varphi^{-1}(C)$ ou $D=\varphi^{-1}(B)=A$, o que significa que $\varphi^{-1}(C)$ é maximal.

$\mathrm{O}$ caso $C \unlhd B \Rightarrow \varphi^{-1}(C) \unlhd A$ é maximal, se prova de modo análogo.

Lema 2.3.4. Sejam $A_{1}$ e $A_{2}$ subálgebras báricas de uma álgebra bárica $(A, w)$. Então $A_{1}=A_{2}$ se e somente se $A_{1} \subseteq A_{2}$ e bar $\left(A_{1}\right) \supseteq \operatorname{bar}\left(A_{2}\right)$

Demonstração: A parte somente se é imediata. Agora, note que $A_{1} \subseteq A_{2} \Rightarrow$ $\operatorname{bar}\left(A_{1}\right) \subseteq \operatorname{bar}\left(A_{2}\right)$ e como, por hipótese, $\operatorname{bar}\left(A_{1}\right) \supseteq \operatorname{bar}\left(A_{2}\right)$, resulta $\operatorname{bar}\left(A_{1}\right)=$ $\operatorname{bar}\left(A_{2}\right)$. Daí: $A_{1}=F a \oplus \operatorname{bar}\left(A_{1}\right)=F a \oplus \operatorname{bar}\left(A_{2}\right)=A_{2}, \quad \forall a \in A, w(a)=1$. 
A próxima proposição corresponde a um dos conhecidos teoremas do homomorfismo de álgebras, válidos naturalmente para álgebras báricas. A demonstração se faz do modo usual e será aqui omitida.

Proposição 2.3.5. Sejam B uma subálgebra de uma álgebra bárica $(A, w)$ e I um ideal bárico de $A$. Então $B+I \leq A, I$ é um ideal bárico de $B+I$ e $\frac{B+I}{I} \cong_{b} \frac{B}{I \cap B}$.

Lema 2.3.6. Sejam $(A, w)$ uma álgebra bárica e $B \unlhd A$. Então $B$ é maximal se $e$ somente se bar $(B)$ é um ideal bárico maximal de $(A, w)$.

Demonstração: $(\Rightarrow)$ Seja $B \triangleleft A$ maximal $(B \neq A)$. Quero mostrar que bar $(B)$ é um ideal bárico maximal de $A$. Seja então $D$ um ideal bárico de $A$ tal que $\operatorname{bar}(D) \subseteq$ $D \subseteq \operatorname{bar}(A)$. Seja $b \in B$ tal que $w(b)=1$. Sabemos que $B=F b \oplus \operatorname{bar}(A)$. Fazendo $C=F b \oplus D$, segue que $D=\operatorname{bar}(C)$, que é ideal bárico de $A$, donde $C \unlhd A$. Temos ainda: $B \subseteq C \subseteq A$, e como $B$ é maximal, $C=B$ ou $C=A$. Daí, $\operatorname{bar}(C)=\operatorname{bar}(B)$ ou $\operatorname{bar}(C)=\operatorname{bar}(A)$ e portanto $D=\operatorname{bar}(B)$ ou $D=\operatorname{bar}(A)$.

$(\Leftarrow)$ Suponhamos agora que $\operatorname{bar}(B)$ é um ideal bárico maximal de $A$. Seja $C \unlhd A$ tal que $B \subseteq C$. Assim: $\operatorname{bar}(B) \subseteq \operatorname{bar}(C) \subseteq \operatorname{bar}(A)$, e como $\operatorname{bar}(B)$ é maximal, resulta que $\operatorname{bar}(C)=\operatorname{bar}(B)$ ou $\operatorname{bar}(C)=\operatorname{bar}(A)$. Então, por 2.3.4 devemos ter $C=B$ ou $C=A$, o que indica que $B$ é maximal.

Definição 2.3.5. Uma álgebra bárica $(A, w)$ é simples se para todas $B \unlhd A$, tivermos $\operatorname{bar}(B)=0$ ou $\operatorname{bar}(B)=\operatorname{bar}(A)$.

Lema 2.3.7. Sejam $(A, w)$ uma álgebra bárica e $B \unlhd A$. Então $B$ é maximal se $e$ somente se $A / \operatorname{bar}(B)$ é simples.

Demonstração: Seja $\varphi: A \rightarrow A / \operatorname{bar}(B)$ o epimorfismo bárico dado por $\varphi(a)=$ $a+\operatorname{bar}(B)$. Seja $B \triangleleft A$ maximal. Então, se $C \unlhd A / \operatorname{bar}(B)$, por 2.3.3, temos:

$\varphi^{-1}(C) \unlhd \varphi^{-1}(A / \operatorname{bar}(B))=A . \quad$ E também $\operatorname{bar}(B) \subseteq \operatorname{bar}\left(\varphi^{-1}(C)\right)$. De fato, $b \in \operatorname{bar}(B) \Rightarrow \varphi(B)=b+\operatorname{bar}(B)=0 \in C \Rightarrow b \in \varphi^{-1}(C) \stackrel{w(b)=0}{\Rightarrow} b \in \operatorname{bar}\left(\varphi^{-1}(C)\right)$.

$$
\Rightarrow \operatorname{bar}(B) \subseteq \operatorname{bar}\left(\varphi^{-1}(C)\right)
$$

Mas, pelo Lema 2.3.6, sendo $B \triangleleft A$ maximal, $\operatorname{bar}(B)$ é um ideal bárico maximal de $\operatorname{bar}(A)$. Portanto: 


$$
\operatorname{bar}\left(\varphi^{-1}(C)\right)=\operatorname{bar}(B) \text { ou } \operatorname{bar}\left(\varphi^{-1}(C)\right)=\operatorname{bar}(A)
$$

Mas, podemos escrever $C=\varphi^{-1}(C) / \operatorname{bar}(B)$. Então:

$$
\operatorname{bar}(C)=\operatorname{bar}\left(\varphi^{-1}(C) / \operatorname{bar}(B)\right) \Rightarrow \operatorname{bar}(C)=\operatorname{bar}\left(\varphi^{-1}(C)\right) / \operatorname{bar}(B)
$$

Por (I) temos: $\operatorname{bar}(C)=\operatorname{bar}(B) / \operatorname{bar}(B)=0 \quad$ ou

$$
\operatorname{bar}(C)=\operatorname{bar}(A) / \operatorname{bar}(B)=\operatorname{bar}(A / \operatorname{bar}(B))
$$

Reciprocamente, seja $A / \operatorname{bar}(B)$ simples. Se existe $C \unlhd A$ tal que $B \subseteq C$ então, pelo Lema 2.3.2, $\varphi(C) \unlhd A / \operatorname{bar}(B)$. Mas, sendo $A / \operatorname{bar}(B)$ simples, devemos ter $\operatorname{bar}(\varphi(C))=0$ ou $\operatorname{bar}(\varphi(C))=\operatorname{bar}(A / \operatorname{bar}(B))$. Novamente, por 2.3.2, $\operatorname{bar}(\varphi(C))=$ $\varphi(\operatorname{bar}(C))$. Logo:

$$
\begin{aligned}
& \operatorname{bar}(B)=\varphi^{-1}(0)=\operatorname{bar}(C) \quad \text { ou } \\
& \operatorname{bar}(C)=\varphi^{-1}(\operatorname{bar}(A / \operatorname{bar}(B)))=\operatorname{bar}\left(\varphi^{-1}(A / \operatorname{bar}(B))\right)=\operatorname{bar}(A) .
\end{aligned}
$$

Definição 2.3.6. O bar radical de uma álgebra bárica alternativa $(A, w)$ é a intersecção de todos bar $(B)$, onde $B \triangleleft A$ é maximal. Notação: $R_{B}(A)$.

Lema 2.3.8. Sejam $(A, w)$ uma álgebra bárica e I um ideal bárico de A. Então são válidas:

(i) $R_{B}(A / I)=\bigcap \operatorname{bar}(B) / I$, onde $B \triangleleft A$ é maximal e $I \subseteq B$;

(ii) $\left(R_{B}(A)+I\right) / I \subseteq R_{B}(A / I)$;

(iii) $S e I \subseteq R_{B}(A)$, então $R_{B}(A / I)=R_{B}(A) / I$.

\section{Demonstração:}

(i) Seja $\varphi: A \rightarrow A / I, \varphi(a)=a+I$, epimorfismo bárico. Por definição, $R_{B}(A / I)$ é a intersecção de $\operatorname{bar}(C)$, onde $C \unlhd A / I$ é maximal. Seja $B \triangleleft A$, maximal e com $I \subseteq B$. Podemos fazer o quociente $B / I$ e, é claro que $\varphi(B)=B / I$. Pelo Lema 2.3.3, $\varphi(B)=B / I \unlhd A / I$ e é maximal. Portanto $R_{B}(A / I) \subseteq \bigcap \operatorname{bar}(B / I)$. Seja agora, $B^{\prime} \triangleleft A / I$ maximal. Então, pelo Lema 2.3.3, $\varphi^{-1}\left(B^{\prime}\right) \unlhd \varphi^{-1}(A / I)=A$.

(ii) Se $B \triangleleft A$ é maximal e $I \subseteq B$, então $R_{B}(A)+I \subseteq B$. Logo $R_{B}(A)+$ $I \subseteq \bigcap_{I \subseteq B \triangleleft A} \operatorname{bar}(B)$. Fazendo o quociente por I, teremos: $\left(R_{B}(A)+I\right) / I \subseteq$ $\bigcap_{I \subseteq B \triangleleft A} \operatorname{bar}(B) / I$. Logo, por (i), $\left(R_{B}(A)+I\right) / I \subseteq R_{B}(A / I)$. 
(iii) Se $I \subseteq R_{B}(A)$, então $I \subseteq B, \forall B \triangleleft A$ maximal. Então, por (i), $R_{B}(A / I)=$ $R_{B}(A) / I$.

Lema 2.3.9. Sejam $(A, w)$ uma álgebra bárica e $B \unlhd A$. Então $R_{B}(B) \subseteq R_{B}(A) \cap B$.

Demonstração: Tomemos $C \triangleleft A$ maximal. Se $\operatorname{bar}(B) \subseteq \operatorname{bar}(C)$, então $R_{B}(B) \subseteq$ $B \cap \operatorname{bar}(C)$. De fato, $R_{B}(B) \subseteq \operatorname{bar}(B)=B \cap \operatorname{bar}(B) \subseteq B \cap \operatorname{bar}(C)$.

Agora, se $\operatorname{bar}(B) \nsubseteq \operatorname{bar}(C)$, então $\operatorname{bar}(B) \nsubseteq C, \operatorname{logo} C \subset C+\operatorname{bar}(B)$. Mas $C$ é maximal, portanto $A=C+\operatorname{bar}(B)$.

Seja $x \in B$. Como $B \subseteq A, x \in A$. Logo posso escrever $x=c+b$, onde $c \in C$ e $b \in \operatorname{bar}(B)$. Daí, $c=x-b \Rightarrow c \in B \Rightarrow c \in B \cap C$. Com isto $x \in B \cap C+\operatorname{bar}(B)$, o que resulta em: $B \subseteq B \cap C+\operatorname{bar}(B)$. Como a inclusão contrária é óbvia, obtemos: $B=B \cap C+\operatorname{bar}(B)$.

Tomamos agora $b \in B \cap C$ tal que $w(b)=1$ e escrevemos: $C=F b+\operatorname{bar}(C)$ e também $B=F b+\operatorname{bar}(B)$. Mas

$$
A=C+\operatorname{bar}(B)=F b+\operatorname{bar}(C)+\operatorname{bar}(B) \Rightarrow A=B+\operatorname{bar}(C) \text {. Então: }
$$

$$
\frac{A}{\operatorname{bar}(C)}=\frac{B+\operatorname{bar}(C)}{\operatorname{bar} C} \stackrel{(*)}{\cong}_{b} \frac{B}{\operatorname{bar}(C) \cap B}=\frac{B}{\operatorname{bar}(C \cap B)}
$$

(*) Prop. 2.3.5

Como $C \triangleleft A$ é maximal, pelo Lema 2.3.7, $A / \operatorname{bar}(C)$ é simples, daí, pelo isomorfismo indicado acima, também $B \cap C \triangleleft B$ é maximal. Temos ainda: $R_{B}(B) \subseteq \operatorname{bar}(B \cap C)=$ $B \cap \operatorname{bar}(C)$. Como $C$ é arbitrária, esta inclusão vale para toda subálgebra maximal de $A$. Então:

$$
R_{B}(B) \subseteq \bigcap_{C \triangleleft A}(B \cap \operatorname{bar}(C))=B \cap\left(\bigcap_{C \triangleleft A} \operatorname{bar}(C)\right)=B \cap R_{B}(A)
$$

Proposição 2.3.10. Sejam $(A, w)$ álgebra bárica e $B$, subálgebra bárica normal de A tal que $R_{B}(A / \operatorname{bar}(B))=0$. Então $R_{B}(B) \subseteq R_{B}(A) \subseteq B$.

Demonstração: Lembrando que $\operatorname{bar}(B)$ é um ideal bárico de $A$, podemos aplicar o Lema 2.3.8 (ii), obtendo:

$$
\left(R_{B}(A)+\operatorname{bar}(B)\right) / \operatorname{bar}(B) \subseteq R_{B}(A / \operatorname{bar}(B))=0 \Rightarrow R_{B}(A) \subseteq \operatorname{bar}(B) \subseteq B
$$

Assim, $R_{B}(A) \cap B=R_{B}(A)$ e, pelo Lema 2.3.9, $R_{B}(B) \subseteq R_{B}(A)$ 
Definição 2.3.7. Seja $(A, w)$ uma álgebra bárica alternativa tal que $R_{B}(A)=0$. Dizemos que $(A, w)$ é semisimples no sentido bárico ou b-semisimples.

Definição 2.3.8. Seja $(A, w)$ uma álgebra bárica alternativa e I um ideal bárico de A. Se I for simples, dizemos que I é um ideal b-simples.

A estrutura das álgebras báricas b-semisimples, semelhantemente às álgebras semisimples, é dada como uma soma direta de ideais b-simples. O teorema a seguir explicita tal resultado. Omitiremos sua demonstração, que pode ser encontrada em $[7]$.

Teorema 2.3.11. Seja $(A, w)$ álgebra bárica de dimensão finita. Então $A$ é bsemisimples se e somente se bar $(A)$ é soma direta de ideais b-simples.

Lema 2.3.12. Seja $(A, w)$ álgebra bárica alternativa e $R(A)$ o radical de $A$. Se $R(A)=0$, então $R_{B}(A)=0$.

Demonstração: Se $R(A)=0$, sabemos que $A=G_{1} \oplus \cdots \oplus G_{k}$, com os $G_{i}$ ideais simples. Cada $G_{i}$ tem um elemento identidade $e_{i}$ e $G_{i}=e_{i} A$. Para cada $e_{i}$ tem-se $w\left(e_{i}\right)=0$ ou $w\left(e_{i}\right)=1$. Mas necessariamente, para algum $i, w\left(e_{i}\right)=1$, caso contrário $G_{i} \subseteq \operatorname{bar}(A)$ e $A$ não seria uma álgebra bárica. Supondo sem perda de generalidade que $w\left(e_{1}\right)=1$, temos que $w\left(e_{j}\right)=0$ para $1<j \leq k$. Assim $G_{j} \subseteq \operatorname{bar}(A)$, se $1<j \leq$ $k$. Já vimos que $G_{1}=F e_{1} \oplus \operatorname{bar}\left(G_{1}\right)$. Mas $\operatorname{bar}\left(G_{1}\right)$ é um ideal de $A$, e $\operatorname{bar}\left(G_{1}\right) \subseteq G_{1}$. Como $G_{1}$ é simples, segue que $\operatorname{bar}\left(G_{1}\right)=0$. Assim: $A=F e_{1} \oplus G_{2} \oplus \cdots \oplus G_{k}$ ou $A=F e_{1} \oplus \operatorname{bar}(A)$. Portanto, pelo Teorema 2.3.11, $R_{B}(A)=0$.

Teorema 2.3.13. Seja $(A, w)$ álgebra bárica alternativa. Então $R_{B}(A) \subseteq R(A)$.

Demonstração: Por definição, $R_{B}(A)=\bigcap_{B \triangleleft A} \operatorname{bar}(B)$. Claramente, $R_{B}(A) \subseteq \operatorname{bar}(A)$. Se $\operatorname{bar}(A)=R(A)$, então $R_{B}(A) \subseteq R(A)$. Se $\operatorname{bar}(A) \neq R(A)$, consideramos a subálgebra $P$ dada por $P=F e \oplus R(A)$, onde $e$ é um idempotente de peso 1 de $A$ (existe $e$ pelo Corolário 1.4.6). $P$ é normal, pois $\operatorname{bar}(P)=R(A)$ é ideal de $A$. Como, pelo Lema 2.1.17, $R(A / R(A))=0$ segue, pelo Lema 2.3.12, que $R_{B}(A / R(A))=0$. Podemos então aplicar a Proposição 2.3.10, com $R(A)$ no lugar de $B$, e chegamos a $R_{B}(A) \subseteq R(A)$. 
Proposição 2.3.14. Seja $(A, w)$ álgebra bárica, com bar $(A)=I_{1} \oplus \cdots \oplus I_{k}$, com os $I_{i}$ ideais báricos simples. Se $J$ é qualquer ideal bárico de $A$, então: $J=0$ ou $J=I_{j_{1}} \oplus \cdots \oplus I_{j_{l}}, 1 \leq j_{i} \leq k$.

Demonstração: Seja $J$ ideal bárico de $A$. Então $J \subseteq \operatorname{bar}(A)$ e, se $j \in J$ podemos escrever $j=i_{1}+\cdots+i_{k}$, com $i_{l} \in I_{l}, \quad 1 \leq l \leq k$ (podendo algum $i_{l}$ ser zero). Consideremos o seguinte conjunto:

$$
J_{l}=\left\{i_{l} \in I_{l} \text { tal que } i_{l} \text { é somando de algum } j \in J\right\}
$$

Mostremos que $J_{l}$ é um ideal de $A$. Seja $x \in A$. Provemos que $i_{l} x, x i_{l} \in J_{l}, \forall i_{l} \in$ $J_{l}$. Tomemos $i_{l} \in J_{l}$ arbitrário. Então existe $j \in J$ tal que $i_{l}$ é um somando de j. Como $J$ é ideal, $x j, j x \in J$. E como $I_{l}$ é ideal, $x i_{l}, i_{l} x \in I_{l}$. Mas $x i_{l} \in I_{l}$ e é somando de $x j \in J$. $\operatorname{Logo} x i_{l} \in I_{l}$. Analogamente temos $i_{l} \in J_{l}$. Portanto $J_{l}$ é um ideal de $A$. Cada $J_{l} \subseteq I_{l}$, $\operatorname{logo} J_{l}=0$ ou $J_{l}=I_{l}$, pois $I_{l}$ é um ideal simples. E como $J \subseteq J_{1} \oplus \cdots \oplus J_{j}, 1 \leq j \leq k$, segue que $J=I_{1} \oplus \cdots \oplus I_{j}$.

Proposição 2.3.15. Sejam $(A, w)$ álgebra bárica alternativa e I um ideal b-simples de A. Então $I^{2}=I$ ou $I^{2}=0$.

Demonstração: Como $I^{2} \subseteq I$, e $I$ é um ideal simples, basta provar que $I^{2}$ é um ideal de $A$. Tomamos $x \in A$ e $y=a b \in I^{2} \quad(a, b \in I)$

$$
\begin{gathered}
x(a b)=(x a) b-(x, a, b)=(x a) b=(b, x, a)=(x a) b-(b x) a+b(x a) \in I^{2} \\
(a b) x=(a, b, x)+a(b x)=-(a, x, b)+a(b x)=a(x b)-(a x) b+a(b x) \in I^{2}
\end{gathered}
$$

Portanto, $I^{2}$ é um ideal de $A$.

Lema 2.3.16. Seja $(A, w)$ uma álgebra bárica alternativa b-semisimples. Então $A$ pode ser escrita:

$$
A=F e \oplus R_{1} \oplus \cdots \oplus R_{m} \oplus I_{1} \oplus \cdots \oplus I_{p}
$$

onde os ideais b-simples $R_{i}$ e $I_{j}$ satisfazem $R_{i}^{2}=0$ e $I_{j}^{2}=I_{j}, 1 \leq i \leq m$ e $1 \leq j \leq p$.

Demonstração: É conseqüência direta do Teorema 2.3.11 e da Proposição 2.3.15. 
Proposição 2.3.17. Com as mesmas hipóteses do lema anterior, a subálgebra bárica alternativa $A^{\prime}=F e \oplus I_{1} \oplus \cdots \oplus I_{p}$ é semisimples, isto é, $R\left(A^{\prime}\right)=0$ e existe um idempotente $e_{1} \in A$ com $w\left(e_{1}\right)=1$ e tal que $e_{1} I_{l}=I_{l} e_{1}=0,1 \leq l \leq p$.

Demonstração: Seja $x \in R\left(A^{\prime}\right)$. Então $x$ é propriamente nilpotente em A'. Então, para todo $y \in R(A)=R_{1} \oplus \cdots \oplus R_{m}, x y=0$. Assim, $x$ é propriamente nilpotente em $A$, pois $A=A^{\prime} \oplus R(A)$. Logo $R\left(A^{\prime}\right) \subset R(A)$, daí $R\left(A^{\prime}\right) \subset R(A) \cap A^{\prime}=\{0\}$. Portanto $A^{\prime}$ é semisimples.

Pelo Teorema 2.1.16, $A^{\prime}$ pode ser escrita como soma direta de ideais simples: $A^{\prime}=G_{1} \oplus \cdots \oplus G_{r}$, onde cada $G_{i}$ é ideal simples $(i=1, \ldots, r)$. O idempotente $e \in A^{\prime}$ pode ser escrito: $e=e_{1}+\cdots+e_{r}$, onde cada $e_{i} \in G_{i}$ é idempotente. Como $e$ tem peso 1 , somente um dos $e_{i}$ tem peso 1 , e os outros têm peso 0 . Suponhamos que $w\left(e_{1}\right)=1$.

$\operatorname{bar}\left(G_{1}\right)$ é um ideal de $G_{1}$, logo é também ideal de $A$. Como $G_{1}$ é simples, então $\operatorname{bar}\left(G_{1}\right)=G_{1}$ ou $\operatorname{bar}\left(G_{1}\right)=0$. Mas $e_{1} \in G_{1}$ tem peso $1, \operatorname{logo} \operatorname{bar}\left(G_{1}\right)=0$. Assim $G_{1}=F e_{1}$. Escrevemos então:

$A^{\prime}=F e_{1} \oplus G_{2} \oplus \cdots \oplus G_{r}$. Como a soma é direta, temos que $e_{1} G_{i}=G_{i} e_{1}=0$, para $2 \leq i \leq r$.

Por hipótese, $A^{\prime}=F e_{1} \oplus I_{1} \oplus \cdots \oplus I_{p}$. Comparando as duas decomposições de $A^{\prime}$ temos:

$$
G_{2} \oplus \cdots \oplus G_{r}=I_{1} \oplus \cdots \oplus I_{p}
$$

Cada $G_{i}$ é um ideal bárico de $A^{\prime}$, pois $G_{i} \subseteq \operatorname{bar}\left(A^{\prime}\right)$, logo, de acordo com a Proposição 2.3.14, $G_{i}$ é uma soma dos ideais $I_{l}$. Mas os ideais $I_{l}$ são simples, logo cada $G_{i}$ é igual a um certo $I_{l}$. Portanto $e_{1} I_{l}=I_{l} e_{1}=0$.

Vamos procurar uma relação mais explícita entre $R_{B}(A)$ e $R(A)$. Veremos que, o $\operatorname{bar}(A)$ representa um papel importante neste caso.

Teorema 2.3.18. Seja $(A, w)$ álgebra bárica alternativa. Então

$$
R_{B}(A)=\operatorname{bar}(A)^{2} \cap R(A)
$$

Demonstração: Mostremos primeiro que $R_{B}(A) \subseteq \operatorname{bar}(A)^{2} \cap R(A)$. Já sabemos que $R_{B}(A) \subseteq R(A)$, portanto basta mostrar que $R_{B}(A) \subseteq \operatorname{bar}(A)^{2}$. Para isto consideremos a decomposição de Peirce de $A$ em relação a um idempotente $e \in A$. 


$$
A=F e \oplus \operatorname{bar}(A)=F e \oplus \operatorname{bar}(A)_{11} \oplus \operatorname{bar}(A)_{10} \oplus \operatorname{bar}(A)_{01} \oplus \operatorname{bar}(A)_{00}
$$

Como $\operatorname{bar}(A) \subseteq \operatorname{bar}(A)^{2}$ podemos escrever esta decomposição em termos de subespaços $X_{i j} \subseteq \operatorname{bar}(A)_{i j}$, da seguinte forma:

$$
A=F e \oplus \operatorname{bar}(A)^{2} \oplus X_{11} \oplus X_{10} \oplus X_{01} \oplus X_{00}
$$

Sejam $Y_{i j}$ bases dos subespaços $X_{i j}$ e tomemos o seguinte conjunto:

$$
J_{z}=\operatorname{bar}(A)^{2}+\left\langle Y_{11} \cup Y_{10} \cup Y_{01} \cup Y_{00} \backslash\{z\}\right\rangle
$$

onde $z \in Y_{11} \cup Y_{10} \cup Y_{01} \cup Y_{00}$.

Vamos mostrar que $J_{z}$ é um ideal bárico maximal de $A$. Em primeiro lugar provemos que $J_{z}$ é um ideal. Daí segue que $J_{z}$ é bárico, uma vez que $J_{z} \subseteq \operatorname{bar}(A)$. Sejam $a \in A$ e $j \in J_{z}$. Se $a \in \operatorname{bar}(A), a j_{z}, j_{z} a \in \operatorname{bar}(A)^{2}, \operatorname{logo} a j_{z}, j_{a} z \in J_{z}$. Resta mostrar que $e j_{z}, j_{z} e \in J_{z}$.

Um elemento genérico $x \in \operatorname{bar}(A)^{2}$ pode ser escrito como $x=\sum a_{i} b_{i}, \operatorname{com} a_{i}, b_{i} \in$ $\operatorname{bar}(A)$. Então se $j_{z} \in J_{z}$, podemos escrever:

$$
\begin{gathered}
j_{z}=\sum a_{i} b_{i}+a_{11}+a_{10}+a_{01}+a_{00}, \quad \text { onde } a_{i j} \in Y_{i j} \\
e j_{z}=\sum e\left(a_{i} b_{i}\right)+e a_{11}+e a_{10}+e a_{01}+e a_{00}=\sum e\left(a_{i} b_{i}\right)+a_{11}+a_{10}
\end{gathered}
$$

Mas, a parcela $\sum e\left(a_{i} b_{i}\right)$ está em $\operatorname{bar}(A)^{2}$, como podemos verificar, calculando:

$$
\begin{aligned}
e\left(a_{i} b_{i}\right) & =\left(e a_{i}\right) b_{i}-\left(e, a_{i}, b_{i}\right)=\left(e a_{i}\right) b_{i}+\left(a_{i}, e, b_{i}\right) \\
& =\left(e a_{i}\right) b_{i}+\left(a_{i} e\right) b_{i}-a_{i}\left(e b_{i}\right) \in \operatorname{bar}(A)^{2}
\end{aligned}
$$

Como a codimensão de $J_{z}$ é 1 , segue que $J_{z}$ é maximal. Sendo isto válido para todo $z \in Y_{11} \cup Y_{10} \cup Y_{01} \cup Y_{00}$ temos:

$$
R_{B}(A) \subseteq \bigcap_{z} J_{z}=\operatorname{bar}(A)^{2}
$$

Então: $R_{B}(A) \subseteq \operatorname{bar}(A)^{2} \Rightarrow R_{B}(A) \subseteq \operatorname{bar}(A)^{2} \cap R(A)$

Devemos mostrar agora que $\operatorname{bar}(A)^{2} \cap R(A) \subseteq R_{B}(A)$. Para isto vamos considerar a álgebra quociente $A / R_{B}(A)$. Já vimos que $R_{B}\left(A / R_{B}(A)\right)=0$, isto é $A / R_{B}(A)$ é semisimples no sentido bárico. Sendo assim, ela pode ser escrita como soma direta de ideais báricos simples. Em particular: 


$$
\operatorname{bar}\left(A / R_{B}(A)\right)=I_{1} \oplus \cdots \oplus I_{r} \oplus J_{1} \oplus \cdots \oplus J_{s}
$$

onde $I_{i}^{2}=I_{i}, \quad(1 \leq i \leq r)$, e $J_{j}^{2}=0, \quad(1 \leq j \leq s)$.

Notemos agora que $\operatorname{bar}\left(A / R_{B}(A)\right)^{2}=I_{1} \oplus \cdots \oplus I_{r}$. De fato:

$$
\begin{aligned}
\operatorname{bar} & \left(A / R_{B}(A)\right)^{2}=\operatorname{bar}\left(A / R_{B}(A)\right) \cdot \operatorname{bar}\left(A / R_{B}(A)\right) \\
& =\left(I_{1} \oplus \cdots \oplus I_{r} \oplus J_{1} \oplus \cdots \oplus J_{s}\right) \cdot\left(I_{1} \oplus \cdots \oplus I_{r} \oplus J_{1} \oplus \cdots \oplus J_{s}\right) \\
& =I_{1}^{2} \oplus \cdots \oplus I_{r}^{2} \oplus J_{1}^{2} \oplus \cdots \oplus J_{s}^{2} \\
& =I_{1}^{2} \oplus \cdots \oplus I_{r}^{2} \oplus 0 \oplus \cdots \oplus 0 \\
& =I_{1} \oplus \cdots \oplus I_{r}
\end{aligned}
$$

Então, pela Proposição 2.3.17 $\operatorname{bar}\left(A / R_{B}(A)\right)^{2}$ é semisimples no sentido usual, isto é, $R\left(\operatorname{bar}\left(A / R_{B}(A)\right)^{2}\right)=0$.

Mas $\operatorname{bar}\left(A / R_{B}(A)\right)^{2} \cong \operatorname{bar}(A)^{2} /\left(\operatorname{bar}(A)^{2} \cap R_{B}(A)\right)$. De fato:

$$
\begin{aligned}
\operatorname{bar}\left(A / R_{B}(A)\right)^{2} & \cong \operatorname{bar}(A)^{2} / R_{B}(A)^{2}=\operatorname{bar}(A)^{2} / R_{B}(A) \\
& =\operatorname{bar}(A)^{2} /\left(R_{B}(A) \cap \operatorname{bar}(A)^{2}\right) .
\end{aligned}
$$

Esta última igualdade resulta do fato que $R_{B}(A) \subseteq \operatorname{bar}(A)^{2}$, como vimos há pouco, $\log$ o $R_{B}(A) \cap \operatorname{bar}(A)^{2}=R_{B}(A)$. Então:

$$
\begin{aligned}
0 & =R\left(\operatorname{bar}\left(A / R_{B}(A)\right)^{2}\right)=R\left(\operatorname{bar}(A)^{2}\right) / R\left(R_{B}(A) \cap \operatorname{bar}(A)^{2}\right) \\
& =R\left(\operatorname{bar}(A)^{2}\right) / R\left(R_{B}(A)\right)=R\left(\operatorname{bar}(A)^{2}\right) / R_{B}(A) \\
& =R\left(\operatorname{bar}(A)^{2}\right) / R_{B}(A) \cap \operatorname{bar}(A)^{2}
\end{aligned}
$$

$\operatorname{Logo} R\left(\operatorname{bar}(A)^{2}\right) \subseteq R_{B}(A) \cap \operatorname{bar}(A)^{2}=R_{B}(A)$ ou

$$
R\left(\operatorname{bar}(A)^{2}\right) \subseteq R_{B}(A)
$$

Lembrando, estamos querendo mostrar que $R(A) \cap \operatorname{bar}(A)^{2} \subseteq R_{B}(A)$. Mas, é claro que $R(A) \cap \operatorname{bar}(A)^{2} \subseteq \operatorname{bar}(A)^{2}$. E também, $R(A) \cap \operatorname{bar}(A)^{2} \subseteq R(A)$, donde $R(A) \cap \operatorname{bar}(A)^{2}$ é um ideal nil. Estando contido em $\operatorname{bar}(A)^{2}$, deve estar contido no radical de $\operatorname{bar}(A)^{2}$. Assim: $R(A) \cap \operatorname{bar}(A)^{2} \subseteq R\left(\operatorname{bar}(A)^{2}\right)$. E, como acabamos de verificar, $R\left(\operatorname{bar}(A)^{2}\right) \subseteq R_{B}(A)$, segue que $R(A) \cap \operatorname{bar}(A)^{2} \subseteq R_{B}(A)$, completando a demonstração do teorema. 
Corolário 2.3.19. Seja $(A, w)$ álgebra bárica alternativa. Então bar $(A)$ é nilpotente se e somente se $R_{B}(A)=R(A)^{2}$.

Demonstração: $(\Rightarrow)$ Se $\operatorname{bar}(A)$ é nilpotente então $\operatorname{bar}(A)=R(A)$, pois $R(A) \subseteq$ $\operatorname{bar}(A)$ e $R(A)$ é o ideal nilpotente maximal de $A$. Logo

$$
R(A) \cap \operatorname{bar}(A)^{2}=R(A) \cap R(A)^{2}=R(A)^{2}, \text { pois } R(A)^{2} \subseteq R(A)
$$

Como $R(A) \cap \operatorname{bar}(A)^{2}=R_{B}(A)$ segue que $R(A)^{2}=R_{B}(A)$.

$(\Leftarrow)$ Se $R_{B}(A)=R(A)^{2}$, temos $R(A) \cap \operatorname{bar}(A)^{2}=R(A)^{2}$, isto é,

$$
R(A) \cap \operatorname{bar}(A)^{2} \subseteq R(A) .
$$

Logo $\operatorname{bar}(A)$ é nilpotente. 


\section{Capítulo 3}

\section{Álgebras de Composição}

O objetivo deste capítulo é apresentar as principais propriedades das chamadas álgebras de Cayley-Dickson sobre as quais estudaremos algumas identidades no próximo capítulo. A relevância deste estudo reside no fato de que a descoberta destas álgebras, bem como a das álgebras dos quatérnios marcou um passo importante no desenvolvimento da Matemática a partir do século XIX. Foram os quatérnios, álgebras de dimensão 4 descobertos em 1843 por Hamilton, que constituíram o primeiro exemplo de anel com divisão não comutativo. Na mesma época surgiram os octônios (generalizados depois nas álgebras de Cayley-Dickson), de dimensão 8, onde não valia a propriedade associativa.

Intimamente relacionado a estas descobertas está um interessante problema numérico que foi objeto de estudo de matemáticos como Euler e Lagrange. Trata-se do "problema da soma dos quadrados", que pode ser enunciado como:

O produto de somas de $n$ quadrados é ainda uma soma de $n$ quadrados

Para $n=1$ temos a identidade trivial

$$
x^{2} y^{2}=(x y)^{2} .
$$

Para $n=2$, temos a expressão:

$$
\left(x_{1}^{2}+x_{2}^{2}\right)\left(y_{1}^{2}+y_{2}^{2}\right)=\left(x_{1} y_{1}-x_{2} y_{2}\right)^{2}+\left(x_{1} y_{2}+x_{2} y_{1}\right)^{2}
$$

Se considerarmos os números complexos $z=x_{1}+i x_{2}$ e $z^{\prime}=y_{1}+i y_{2}$, a expressão acima corresponde à propriedade dos módulos $\left|z z^{\prime}\right|=|z|\left|z^{\prime}\right|$. 
A identidade para $n=4$ já era do conhecimento de Euler e Lagrange. E em 1818, C. F. Digen encontrou a identidade para $n=8$. Mas foi somente em 1898 que Hurwitz mostrou que tais identidades existem apenas para $n=1,2,4,8$.

O "Problema de Hurwitz" (isto é, a procura dos valores de $n$ para os quais existem as identidades mencionadas), pode ser expresso matematicamente da seguinte forma:

$$
\left(\sum_{i=1}^{n} x_{i}^{2}\right)\left(\sum_{i=1}^{n} y_{i}^{2}\right)=\left(\sum_{i=1}^{n} z_{i}^{2}\right),
$$

onde $z_{i}$ tem a forma

$$
z_{i}=\sum_{j, k=1}^{n} a_{i j k} x_{j} y_{k}
$$

com $a_{i j k}$ números complexos.

Veremos também neste capítulo como se dá a relação das álgebras de composição com o problema numérico estudado por Hurwitz, em que, da mesma forma que só existem 4 valores de $n$ para o problema da soma de quadrados, são igualmente 4 o número de álgebras de composição existentes, a menos de isomorfismos.

Para resultados mais gerais, pode-se usar a abordagem com representação de grupos, conforme esboçado no exemplo 4 da página 49. Maiores detalhes podem ser encontrados em [11].

\subsection{A descoberta dos quatérnios}

Por volta de 1830, William Rowen Hamilton (1805-1865) estabeleceu a fundamentação teórica para os números complexos, representando-os como pares ordenados de números reais. Além da forma usual $a+b i$, passou-se a utilizar também a forma $(a, b)$. Foram definidas as regras das operações de soma e multiplicação de números complexos, que passaram a ser uma ferramenta algébrica importante para se trabalhar com vetores no plano.

Interessava a Hamilton estender as operações e propriedades dos pares ordenados para as ternas ordenadas, de modo a permitir uma aplicação a vetores no espaço. A multiplicação de números complexos tal como formulada por Hamilton e, até hoje em uso, é dada por: 


$$
(a, b)(c, d)=(a c-b d, a d+b c)
$$

ou, na forma algébrica

$$
(a+b i)(c+d i)=a c-b d+(a d+b c) i .
$$

Estendendo este raciocínio, Hamilton tentou definir a multiplicação de ternas de números $(a+b i+c j)(d+e i+f j)$ de tal forma que o resultado também fosse escrito da mesma forma (na linguagem atual, como uma combinação linear de $1, i$ e $j$ ). Mas apareciam os termos $i j$ e $j i$ que não permitiam obter um produto como desejado.

Hamilton também queria preservar para as ternas, a lei dos módulos, válida nos complexos, segundo a qual "o módulo do produto de dois complexos é igual ao produto dos módulos", simbolicamente: $\left|z_{1} z_{2}\right|=\left|z_{1}\right|\left|z_{2}\right|$. Supondo que o produto obtido tivesse a forma dos fatores originais, digamos $x+y i+z j$, esta igualdade seria escrita:

$$
\left(a^{2}+b^{2}+c^{2}\right)\left(d^{2}+e^{2}+f^{2}\right)=x^{2}+y^{2}+z^{2},
$$

que nada mais é do que o problema da soma de quadrados para $n=3$, o qual sabemos hoje, não tem solução.

Após cerca de dez anos de pesquisas, Hamilton percebeu a necessidade de introduzir um novo símbolo, a que chamou $k$, tendo descoberto afinal a relação fundamental entre eles:

$$
\begin{gathered}
i^{2}=j^{2}=k^{2}=-1, \\
i j k=-1 .
\end{gathered}
$$

Destas identidades foram deduzidas as diversas multiplicações entre os símbolos: Multiplicando a segunda igualdade por $k$ à direita:

$$
i j k^{2}=-k \Rightarrow i j=k \text {. }
$$

Multiplicando por $i$ à esquerda:

$$
i^{2} j k=-i \Rightarrow j k=i \text {. }
$$

Procedendo de maneira análoga se chega a:

$$
k i=j, \quad j i=-k, \quad i k=-j, \quad k j=-i .
$$


Hamilton também percebeu que este problema se relacionava com o problema de Hurwitz, mostrando que a existência de uma identidade para a soma de $n$ quadrados era equivalente à existência de uma álgebra de divisão de dimensão $n$ sobre os reais. Maiores detalhes podem ser encontrados em [16].

\subsection{As Álgebras de Composição}

Definição 3.2.1. Seja A um espaço vetorial sobre um corpo arbitrário $F$, com carF $\neq 2$. Uma forma bilinear é uma aplicação $f: A \times A \rightarrow F$ que, para todos $x, x^{\prime}, y, y^{\prime} \in A, \alpha \in F$, satisfaz:

(1) $f\left(x+x^{\prime}, y\right)=f(x, y)+f\left(x^{\prime}, y\right)$;

(2) $f\left(x, y+y^{\prime}\right)=f(x, y)+f\left(x, y^{\prime}\right)$;

(3) $f(\alpha x, y)=f(x, \alpha y)=\alpha f(x, y)$.

Uma forma bilinear é dita simétrica se $f(x, y)=f(y, x)$, para todos $x, y \in A$, e anti-simétrica se $f(x, y)=-f(y, x)$, para todos $x, y \in A$.

Definição 3.2.2. Seja A uma álgebra sobre um corpo arbitrário $F$. Uma aplicação $q: A \rightarrow F$ é chamada uma forma quadrática se satisfizer:

(1) $q(\alpha x)=\alpha^{2} q(x)$, para todo $\alpha \in F$ e todo $x \in A$;

(2) a função $f(x, y)=q(x+y)-q(x)-q(y)$ é uma forma bilinear em $A$.

Dizemos que uma forma quadrática admite composição se existir uma operação binária bilinear xy em A tal que $q(x) q(y)=q(x y)$.

Uma forma quadrática é dita não degenerada se $q(x)=0 \Leftrightarrow x=0$.

A álgebra $A$ está munida de uma forma quadrática não degenerada que admite composição. Vamos verificar que é possível definir um produto em $A$ de tal forma que exista uma unidade em $A$.

Lema 3.2.1. Seja A uma álgebra de dimensão finita munida de uma forma quadrática não degenerada q que admite composição. Então existe um novo produto $x * y$ em $A$ tal que A é uma álgebra com 1. 
Demonstração: Como $q$ é não degenerada, existe $v \in A$ tal que $q(v) \neq 0$. Seja $u=$ $v^{2} / q(v)$. Então $q(u)=1, \operatorname{logo} q(x u)=q(u x)=q(x)$. Consideremos os operadores multiplicativos à direita $R_{u}$ e à esquerda $L_{u}$, dados por

$$
x \mapsto R_{u}(x)=x u \text { e } x \mapsto L_{u}(x)=u x .
$$

Pelo fato de $q$ ser não degenerada e $A$ ter dimensão finita, os operadores $R_{u}$ e $L_{u}$ são invertíveis. Assim, definimos o seguinte produto:

$$
x * y=\left(R_{u}^{-1}(x)\right)\left(L_{u}^{-1}(y)\right)
$$

Como $q$ admite composição: $q(x * y)=q\left(\left(R_{u}^{-1}(x)\right)\left(L_{u}^{-1}(y)\right)\right)$

$$
=q\left(R_{u}^{-1}(x)\right) q\left(L_{u}^{-1}(y)\right)
$$

Mas, notando que: $\quad q\left(R_{u}^{-1}(x)\right)=q\left(\left(R_{u}^{-1}(x) u\right)=q\left(R_{u} R_{u}^{-1}(x)\right)=q(x)\right.$

E também: $\quad q\left(L_{u}^{-1}(y)\right)=q\left(u L_{u}^{-1}(y)\right)=q\left(L_{u} L_{u}^{-1}(y)\right)=q(y)$

Teremos, em (1): $\quad q(x * y)=q(x) q(y)$

Temos também: $\quad L_{u}^{-1}\left(u^{2}\right)=L_{u}^{-1}\left(L_{u}(u)\right)=u \quad$ e $\quad R_{u}^{-1}\left(u^{2}\right)=R_{u}^{-1}\left(R_{u}(u)\right)=u$

Resulta então: $\quad x * u^{2}=\left(R_{u}^{-1}(x)\right)\left(L_{u}^{-1}\left(u^{2}\right)\right)=R_{u}^{-1}(x) u=R_{u}\left(R_{u}^{-1}(x)\right)=x$

E ainda: $\quad u^{2} * x=\left(R_{u}^{-1}\left(u^{2}\right)\right)\left(L_{u}^{-1}(x)\right)=u L_{u}^{-1}(x)=L_{u}\left(L_{u}^{-1}(x)\right)=x$

Logo $u^{2}$ é a unidade de $A$ em relação à multiplicação $*$.

Definição 3.2.3. Uma álgebra $A$ com unidade, sobre um corpo $F$ e munida de uma forma quadrática não degenerada q que admite composição em relação ao produto da álgebra é chamada álgebra de composição.

Lema 3.2.2 (Propriedades). Sejam A uma álgebra de composição sobre um corpo $F$ $(\operatorname{car} F \neq 2), q(x)$ uma forma quadrática não degenerada sobre $A$ e $f(x, y)$ a forma bilinear simétrica associada a $q(x)$. Valem as seguintes propriedades:

(i) $f(x, z) q(y)=f(x y, z y)$;

(ii) $q(x) f(y, w)=f(x y, x w)$;

(iii) $f(x, z) f(y, w)=f(x y, z w)+f(x w, z y)$.

\section{Demonstração:}


(i) Em primeiro lugar note que $q(x)=q(x .1)=q(x) q(1) \Rightarrow q(1)=1$.

Linearizando a lei de composição $q(x y)=q(x) q(y)$, substituindo $x$ por $x+z$, temos:

$$
q((x+z) y)=q(x+z) q(y) \Rightarrow q(x y+z y)=q(x+z) q(y)
$$

Lembrando que a forma bilinear simétrica associada à forma quadrática $q(x)$ é dada por:

$$
f(x, z)=q(x+z)-q(x)-q(y) \Rightarrow q(x+z)=f(x, z)+q(x)+q(z)
$$

Substituindo em (1): $\quad q(x y+z y)=f(x, z) q(y)+q(x) q(y)+q(z) q(y)$

$$
\Rightarrow q(x y+z y)=f(x, z) q(y)+q(x y)+q(z y)
$$

Ainda, por (2):

$$
q(x y+z y)=f(x y, z y)+q(x y)+q(z y)
$$

Igualando (3) e (4):

$$
\begin{gathered}
f(x, z) q(y)+q(x y)+q(z y)=f(x y, z y)+q(x y)+q(z y) \\
\Rightarrow f(x, z) q(y)=f(x y, z y)
\end{gathered}
$$

(ii) Fazendo a linearização, substituindo $y$ por $y+w$, temos:

$$
\begin{aligned}
& q(x(y+w))=q(x) q(y+w) \Rightarrow q(x y+x w)=q(x) q(y+w) \\
& f(y, w)=q(y+w)-q(y)-q(w) \Rightarrow q(y+w)=f(y, w)+q(y)+q(w)
\end{aligned}
$$

Substituindo em (5):

$$
q(x y+x w)=q(x) f(y, w)+q(x y)+q(x w)
$$

Por outro lado:

$$
q(x y+x w)=f(x y, x w)+q(x y)+q(x w)
$$

Igualando (7) e (8):

$$
\begin{gathered}
q(x) f(y, w)+q(x y)+q(x w)=f(x y, x w)+q(x y)+q(x w) \\
\Rightarrow q(x) f(y, w)=f(x y, x w)
\end{gathered}
$$

(iii) Linearizando (I) em relação a $y$, substituindo $y$ por $y+w$ :

$$
\begin{aligned}
f(x, z) q(y+w) & =f(x(y+w), z(y+w))=f(x y+x w, z y+z w) \\
& =f(x y, z y)+f(x y, z w)+f(x w, z y)+f(x w, z w)
\end{aligned}
$$


Por outro lado:

$f(x, z) q(y+w)=f(x, z) f(y, x)+f(x, z) q(y)+f(x, z) q(w)$

Aplicando (I) no segundo membro de (9) e comparando com (10), obtemos:

$$
f(x, z) f(y, w)=f(x y, z w)+f(x w, z y)
$$

Definição 3.2.4. Seja A uma álgebra. Uma involução em A é um operador linear $\rho: A \rightarrow A$, satisfazendo:

(i) $\rho(\rho(x))=x$;

(ii) $\rho(x y)=\rho(y) \rho(x)$, para todos $x, y \in A$.

Observação: A definição acima refere-se a uma involução do $1^{\circ}$ tipo. Outros tipos de involução podem ser encontrados em [22].

Lema 3.2.3. Seja A uma álgebra de composição. A função dada por $x \mapsto \bar{x}=$ $f(x, 1) .1-x$ é uma involução em A. Tem-se também: $x \bar{x}=q(x) .1=\bar{x} x$

Demonstração: Mostremos primeiro que $\bar{x}$ é uma involução. Para isto precisamos mostrar que: (i) $\overline{\bar{x}}=x \quad$ e $\quad$ (ii) $\overline{x y}=\bar{y} \bar{x}$

(i) $\overline{\bar{x}}=f(\bar{x}, 1)-\bar{x}=f(f(x, 1)-x, 1)-f(x, 1)+x$

$$
=f(f(x, 1), 1)-f(x, 1)-f(x, 1)+x=f(x, 1) f(1,1)-2(f(x, 1))+x
$$

Mas $f(1,1)=q(1+1)-q(1)-q(1)=q(2)-2=q(2.1)-2=4 q(1)-2=4-2=2$.

$\operatorname{Logo} \overline{\bar{x}}=2 f(x, 1)-2 f(x, 1)+x \Rightarrow \overline{\bar{x}}=x$.

(ii) $f(\overline{x y}, z)=f(f(x y, 1)-x y, z)=f(f(x y, 1), z)-f(x y, z)$

$$
\begin{gathered}
=f(x y, 1) f(1, z)-f(x y, z) \\
=f(x y, z)+f((x y) z, 1)-f(x y, z)=f(1,(x y) z) \\
f(\bar{y} \bar{x}, z)=f((f(y, 1)-y)(f(x, 1)-x), z) \\
=f(f(y, 1) f(x, 1)-f(y, 1) x-f(x, 1) y+y x, z) \\
=f(f(y, 1) f(x, 1), z)-f(f(y, 1) x, z)-f(f(x, 1) y, z)+f(y x, z) \\
=f(x, 1) f(y, 1) f(1, z)-f(y, 1) f(x, z)-f(x, 1) f(y, z)+f(y x, z)
\end{gathered}
$$




$$
\begin{aligned}
= & f(x y, 1) f(1, z)+f(x, y) f(1, z)-f(y x, z)-f(y z, x)-f(x y, z)-f(x z, y) \\
& +f(y x, z) \\
= & f(x y, z)+f((x y) z, 1)+f(x, y z)+f(x z, y)-f(y z, x)-f(x y, z)-f(x z, y)
\end{aligned}
$$

Logo $f(\bar{y} \bar{x}, z)=f(1,(x y) z)$ e assim:

$f(\overline{x y}, z)=f(\bar{y} \bar{x}, z) \Rightarrow f(\overline{x y}, z)-f(\bar{y} \bar{x}, z)=0 \Rightarrow f(\overline{x y}-\bar{y} \bar{x}, z)=0, \forall z \in A$

Como $f$ é não degenerada, segue que: $\quad \overline{x y}-\bar{y} \bar{x}=0 \Rightarrow \overline{x y}=\bar{y} \bar{x}$

Provemos agora que $x \bar{x}=q(x) \cdot 1=\bar{x} x$. Para isto calculamos:

$$
\begin{aligned}
f(x, \bar{y} z) & =f(x,(f(y, 1) z-y z)=f(x, f(y, 1) z)-f(x, y z) \\
& =f(y, 1) f(x, z)-f(x, y z)
\end{aligned}
$$

Pela igualdade (III) do lema anterior, temos:

$f(x, \bar{y} z)=f(y x, z)+f(y z, x)-f(x, y z) \Rightarrow f(x, \bar{y} z)=f(y x, z)$

Trocando $x$ por $z$ e $z$ por $x$ na igualdade acima, obtemos:

$$
f(y z, x)=f(z, \bar{y} x)
$$

Por (II) e (IV): $\quad q(x) f(1, y)=f(x, x y)=f(\bar{x} x, y)$

Assim:

$$
\begin{aligned}
& f(q(x) \cdot 1, y)=f(\bar{x} x, y) \Rightarrow f(q(x) \cdot 1, y)-f(\bar{x} x, y)=0 \\
& \Rightarrow f(q(x) \cdot 1-\bar{x} x, y)=0, \forall y \in A
\end{aligned}
$$

Logo, como $f$ é não degenerada, temos: $q(x) \cdot 1-\bar{x} x=0 \Rightarrow q(x) \cdot 1=\bar{x} x$.

Mas $q(\bar{x})=q(x)$. De fato:

$$
\begin{aligned}
q(\bar{x}) & =q(f(x, 1)-x)=q(f(x, 1) .1-x)=f(f(x, 1) .1,-x)+q(f(x, 1) .1)+q(-x) \\
& =f(x, 1) f(1,-x)+f(x, 1) f(x, 1) q(1)+q(x) \\
& =-f(x, 1) f(x, 1)+f(x, 1) f(x, 1)+q(x)=q(x)
\end{aligned}
$$

Temos também: $q(\bar{x})=\overline{\bar{x}} \bar{x}=x \bar{x} \Rightarrow x \bar{x}=\bar{x} x$ 
Lema 3.2.4. Seja A uma álgebra de composição em que foi definida uma involução $j(x)=\bar{x}$. Então as seguintes propriedades são verificadas, para todos $x, y \in A$ :

(i) $\bar{x}(x y)=(\bar{x} x) y$;

(ii) $(y x) \bar{x}=y(x \bar{x})$.

\section{Demonstração:}

(i) Calculamos $f(\bar{x}(x y), z)=f((f(x, 1)-x)(x y), z)=f(f(x, 1)(x y)-x(x y), z)$

$$
\begin{aligned}
& =f(f(x, 1)(x y), z)-f(x(x y), z) \\
& =f(x, 1) f(x y, z)-f(x(x y), z) \\
& =f(x(x y), z)+f(x z, x y)-f(x(x y), z) \\
& =q(x) f(z, y)=q(x) f(y, z)=f(q(x) y, z)
\end{aligned}
$$

Assim: $\quad f(\bar{x}(x y), z)=f(q(x) y, z) \Rightarrow f(\bar{x}(x y), z)-f(q(x) y, z)=0$

$$
\Rightarrow f(\bar{x}(x y)-q(x) y, z)=0, \forall z \in A
$$

Como $f$ é não degenerada, obtemos: $\bar{x}(x y)-q(x) y=0 \Rightarrow \bar{x}(x y)=q(x) y$, $\operatorname{mas} q(x)=\bar{x} x, \log \mathrm{o}: \bar{x}(x y)=(\bar{x} x) y$.

(ii) Calculamos $f(z,(y x) \bar{x})=f(z,(y x)(f(x, 1)-x))=f(z, f(x, 1)(y x)-(y x) x)$

$$
\begin{aligned}
& =f(z, f(x, 1)(y x))-f(z,(y x) x) \\
& =f(x, 1) f(z, y x)-f(z,(y x) x) \\
& =f(z, y x) f(x, 1)-f(z,(y x) x) \\
& =f(z x, y x)+f(z,(y x) x)-f(z,(y x) x) \\
& =f(z, y) q(x)=f(z, y q(x))=f(z, y(x \bar{x}))
\end{aligned}
$$

Assim: $\quad f(z,(y x) \bar{x})=f(z, y(x \bar{x})) \Rightarrow f(z,(y x) \bar{x}-y(x \bar{x}))=0, \forall z \in A$

Como $f$ é não degenerada, temos: $(y x) \bar{x}-y(x \bar{x})=0 \Rightarrow(y x) \bar{x}=y(x \bar{x})$.

Teorema 3.2.5. Toda álgebra de composição é alternativa.

Demonstração: Seja $A$ uma álgebra de composição. Pelo Lema 3.2.4, podemos escrever $(\bar{x}, x, y)=0=(y, x, \bar{x})$. Daí:

$$
\begin{aligned}
0 & =(f(x, 1)-x, x, y)=(f(x, 1), x, y)-(x, x, y)=f(x, 1)(1, x, y)-(x, x, y) \\
& =0-(x, x, y) \Rightarrow(x, x, y)=0
\end{aligned}
$$


Analogamente: $0=(y, x, \bar{x})=(y, x, f(x, 1)-x)=(y, x, f(x, 1))-(y, x, x)$

$$
=0-(y, x, x) \Rightarrow(y, x, x)=0
$$

Portanto $A$ é alternativa.

Teorema 3.2.6. Seja A uma álgebra alternativa com 1, em que está definida uma involução $j: x \mapsto \bar{x}$ tal que $x \bar{x}=q(x) .1$, onde $q(x)$ é uma forma quadrática não degenerada. Então A é uma álgebra de composição.

Demonstração: Basta mostrar que $q(x)$ admite composição, i.e. $q(x y)=q(x) q(y)$. Linearizando $x \bar{x}=q(x) 1$, substituindo $x$ por $x+y$, temos:

$$
\begin{aligned}
& (x+y)(\overline{x+y})=q(x+y) \Rightarrow(x+y)(\bar{x}+\bar{y})=f(x, y)+q(x)+q(y) \\
& \Rightarrow x \bar{x}+x \bar{y}+y \bar{x}+y \bar{y}=f(x, y)+x \bar{x}+y \bar{y} \Rightarrow x \bar{y}+y \bar{x}=f(x, y) 1
\end{aligned}
$$

Fazendo $y=1$ na igualdade obtida, vem: $x+\bar{x}=f(x, 1) 1$

Denotando $f(x, 1)$ por $t(x)$, temos: $x+\bar{x}=t(x) 1$

Como $A$ é alternativa, e lembrando que: $(\alpha, x, y)=0$, para todo $\alpha \in F$, e todos $x, y \in A$, e ainda, $x+\bar{x} \in F$, calculamos:

$0=(x+\bar{x}, x, y)=(x, x, y)+(\bar{x}, x, y) \Rightarrow(\bar{x}, x, y)=0$. Analogamente:

$0=(y, x, x+\bar{x})=(y, x, x)+(y, x, \bar{x}) \Rightarrow(y, x, \bar{x})=0$.

Calculemos agora:

$$
\begin{aligned}
q(x y) & =(\overline{x y})(x y)=(\bar{y} \bar{x})(x y)=((t(y)-y) \bar{x})(x y)=(t(y) \bar{x}-y \bar{x})(x y) \\
& =t(y) \bar{x}(x y)-(y \bar{x})(x y)
\end{aligned}
$$

Pelo Lema 3.2.4, $\bar{x}(x y)=(\bar{x} x) y=q(x) y$ e, pela identidade (ii) de Moufang (Teorema 1.2.2), $(y \bar{x})(x y)=y(\bar{x} x) y$. Daí:

$$
\begin{aligned}
q(x y) & =t(y) q(x) y-y(x \bar{x}) y=t(y) q(x) y-q(x) y^{2}=q(x) y(t(y)-y) \\
& =(q(x) y) \bar{y}=q(x) y \bar{y}=q(x) q(y)
\end{aligned}
$$

\subsection{Processo de Duplicação de Cayley-Dickson}

Quais são as álgebras de composição? Como obtê-las? Veremos um processo que permite a construção de tais álgebras a partir de uma álgebra $A$ com 1 , onde está definida uma involução $x \mapsto \bar{x}$ tal que $x \bar{x}=q(x) 1$, sendo $q(x)$ uma forma quadrática não degenerada. Supomos inicialmente que $A$ é alternativa. Pelo Teorema 3.2.6 temos, em $A, x \bar{y}+y \bar{x}=f(x, y) 1$ e também $x+\bar{x}=t(x) 1$, onde $t(x)=f(x, 1)$. 
Seja $\alpha \in F, \alpha \neq 0$. Vamos construir com $A, j$ e $\alpha$ uma álgebra $A_{1}$ satisfazendo as mesmas condições de $A$ e tal que $\operatorname{dim}_{F} A_{1}=2 \operatorname{dim}_{F} A$. Seja $A_{1}=A \oplus A$ o espaço vetorial formado pelos pares ordenados $(x, y)$, com $x, y \in A$. Evidentemente $\operatorname{dim}_{F} A_{1}=2 \operatorname{dim}_{F} A$. Para que $A_{1}$ se torne uma álgebra devemos definir um produto em $A_{1}$. Fazemos:

$$
(x, y)(u, v)=(x u+\alpha v \bar{y}, \bar{x} v+u y)
$$

Com este produto verifica-se facilmente que $A_{1}$ se torna uma álgebra. Além disso, $A_{1}$ tem 1 , que é dado por $(1,0)$. De fato:

$$
\begin{aligned}
& (x, y)(1,0)=(x 1+\alpha \overline{0} y, 0 x+y \overline{1})=(x, y) \\
& (1,0)(x, y)=(1 x+\alpha \bar{y} 0, y 1+0 \bar{y})=(x, y)
\end{aligned}
$$

Podemos identificar $A$ como a subálgebra de $A_{1}$ formada pelos elementos da forma $(x, 0), x \in A$. De fato:

$$
(x, 0)(y, 0)=(x y+\alpha \overline{0} 0,0 x+0 \bar{y})=(x y, 0)
$$

Assim, existe um monomorfismo de $A$ em $A_{1}$ dado por $x \mapsto(x, 0)$.

Seja $e=(0,1) \in A_{1}$. Então $e^{2}=\alpha .1$. De fato:

$$
e^{2}=(0,1)(0,1)=(0.0+\alpha .1 . \overline{1}, \overline{0} .1+0.1)=(\alpha .1,0)=\alpha(1,0)=\alpha .1
$$

Então todo elemento de $A_{1}$ pode ser escrito como:

$$
(x, y)=(x, 0)+(0, y)=x(1,0)+(0,1) y=x .1+e y=x+e y
$$

Consequentemente, denotamos: $A_{1}=A+e A$. É imediato que esta soma é direta, portanto: $A_{1}=A \oplus e A$.

Definimos uma involução em $A_{1}$ da seguinte forma: $j:(x, y) \rightarrow \overline{(x, y)}=(\bar{x},-y)$. Mostremos que $j$, assim definida, é uma involução:

(i) $\overline{\overline{(x, y)}}=\overline{(\bar{x},-y)}=(\overline{\bar{x}},-(-y))=(x, y)$

(ii) $\overline{(x, y)(u, v)}=\overline{(x u+\alpha v \bar{y}, \bar{x} v+u y)}=(\overline{x u+\alpha v \bar{y}},-(\bar{x} v+u y))$

$$
\begin{aligned}
& =(\overline{x u}+\alpha \bar{v} \bar{y},-\bar{x} v-u y)=(\bar{u} \bar{x}+\alpha y \bar{v},-\bar{x} v-u y) \\
& \overline{(u, v)} \overline{(x, y)}=(\bar{u},-v)(\bar{x},-y)=(\bar{u} \bar{x}+\alpha(-y)(-\bar{v}), \overline{\bar{u}}(-y)+\bar{x}(-v)) \\
& =(\bar{u} \bar{x}+\alpha y \bar{v},-\bar{x} v-u y) \\
& \text { Portanto } \overline{(x, y)(u, v)}=\overline{(u, v)} \overline{(x, y)}
\end{aligned}
$$


Vamos agora definir uma forma quadrática não degenerada em $A_{1}$. Como, em $A$, temos $q(x)=x \bar{x}$, vamos usar esta mesma expressão para definir $q(X)$, onde $X=(x, y) \in A_{1}$. Assim, temos:

$$
\begin{aligned}
X \bar{X} & =(x, y) \overline{(x, y)}=(x, y)(\bar{x},-y)=(x \bar{x}+\alpha(-y) \bar{y}, \bar{x}(-y)+\bar{x} y) \\
& =(x \bar{x}-\alpha y \bar{y},-\bar{x} y+\bar{x} y)=(x \bar{x}-\alpha y \bar{y}, 0)=q(x)-\alpha q(y)
\end{aligned}
$$

A forma bilinear associada a $q(X)$ é: $f(X, U)=X \bar{U}+U \bar{X}$, onde $X=(x, y)$ e $U=(u, v)$. Calculamos, então:

$$
\begin{aligned}
f(X, U) & =(x, y) \overline{(u, v)}+(u, v) \overline{(x, y)}=(x, y)(\bar{u},-v)+(u, v)(\bar{x},-y) \\
& =(x \bar{u}+\alpha(-v) \bar{y}, \bar{x}(-v)+\bar{u} y)+(u \bar{x}+\alpha(-y) \bar{v}, \bar{u}(-y)+\bar{x} v) \\
& =(x \bar{u}-\alpha v \bar{y},-\bar{x} v+\bar{u} y)+(u \bar{x}-\alpha y \bar{v}, \bar{u}(-y)+\bar{x} v) \\
& =(x \bar{u}+u \bar{x}-\alpha(v \bar{y}+y \bar{v}), \bar{x} v+\bar{x} v+\bar{u} y-\bar{u} y) \\
& =(x \bar{u}+u \bar{x}-\alpha(v \bar{y}+y \bar{v}), 0)=f(x, u)-\alpha f(v, y)
\end{aligned}
$$

Vamos mostrar que $q(X)$ é não degenerada. Suponhamos $f(X, U)=0, \forall U \in A_{1}$. Então, pela expressão obtida acima:

$$
f(X, U)=(x \bar{u}+u \bar{x}-\alpha(v \bar{y}+y \bar{v}), 0)=0 \Rightarrow x \bar{u}+u \bar{x}-\alpha(v \bar{y}+y \bar{v})=0, \quad \forall u, v \in A .
$$

Em particular, para $v=0$, esta relação continua válida. Daí:

$x \bar{u}+u \bar{x}=0, \quad \forall u \in A$ ou $f(x, u)=0, \quad \forall u \in A$.

Como $f(x, u)$ é não degenerada em $A$, segue que $x=0$.

Fazendo $u=0$, obtemos $-\alpha(v \bar{y}+y \bar{v})=0$, e como $\alpha \neq 0$, resulta $v \bar{y}+y \bar{v}=$ $f(v, y)=0$ ou $f(y, v)=0 \forall v \in A$.

Novamente, como $f(y, v)$ é não degenerada em $A$, devemos ter $y=0$.

Então $X=(x, y)=0$ e $q(X)$ é não degenerada em $A_{1}$.

Obtivemos então uma álgebra $A_{1}$ com as mesmas propriedades da álgebra $A$ e com dimensão duas vezes a de $A$. Denotaremos a álgebra $A_{1}$ por $(A, \alpha)$, enfatizando o escalar usado na construção de $A_{1}$. Mas estamos interessados em obter álgebras de composição. Em que condições a álgebra $(A, \alpha)$ obtida de $A$ pelo processo de duplicação de Cayley-Dickson é uma álgebra de composição? Pelo exposto até agora podemos concluir que basta que $(A, \alpha)$ seja alternativa. Sob que condições isto acontece? É o que provaremos a seguir. Para isto vamos precisar de dois lemas. 
Lema 3.3.1. Em uma álgebra $(A, \alpha)$ obtida de uma álgebra $A$ pelo processo de Cayley-Dickson, tem-se que: $(X, X, Z)=0$ equivale a $(Z, X, X)=0$, para todos $X, Z \in(A, \alpha)$.

Demonstração: Sejam $X, Z \in(A, \alpha)$ tal que $(X, X, Z)=0$. Temos também $(\bar{X}, \bar{X}, \bar{Z})=0$. Então, aplicando involução nesta igualdade temos:

$$
\begin{aligned}
& 0=\overline{(\bar{X}, \bar{X}, \bar{Z})}=\overline{(\bar{X} \bar{X}) \bar{Z}-\bar{X}(\bar{X} \bar{Z})}=\overline{(\bar{X} \bar{X}) \bar{Z}}-\overline{\bar{X}(\bar{X} \bar{Z})} \\
& =\overline{\bar{Z}}(\overline{\bar{X} \bar{X}})-(\overline{\bar{X} \overline{\bar{Z}}}) \overline{\bar{X}}=Z(\overline{\bar{X}} \overline{\bar{X}})-(\overline{\bar{Z}} \overline{\bar{X}}) X \\
& =Z(X X)-(Z X) X=-(Z, X, X)
\end{aligned}
$$

A recíproca se prova de forma inteiramente análoga.

Lema 3.3.2. Seja A uma álgebra alternativa com 1, em que está definida uma involução $\bar{x}$. Então, para todos $x, y \in A,(x, x, y)=0$ se e somente se $(x, \bar{x}, y)=0$.

Demonstração: Lembrando que $\bar{x}=f(x, 1)-x$, e que $f(x, 1) \in F$, temos:

$$
(x, \bar{x}, y)=(x, f(x, 1)-x, y)=(x, f(x, 1), y)-(x, x, y)=-(x, x, y)
$$

Então: $(x, x, y)=0$ se e somente se $(x, \bar{x}, y)=0$.

Podemos agora mostrar o principal resultado desta seção.

Teorema 3.3.3. A álgebra $(A, \alpha)$ obtida de A pelo processo de duplicação de CayleyDickson é alternativa se e somente se $A$ é associativa.

Demonstração: Sejam $X=(x, y)$ e $Z=(z, t) \in(A, \alpha)$. Calculemos:

$$
\begin{aligned}
(X, \bar{X}, Z) & =(X \bar{X}) Z-X(\bar{X} Z)=(x \bar{x}-\alpha(y \bar{y}))(z, t)-(x, y)((\bar{x},-y)(z, t)) \\
& =(q(x)-\alpha q(y))(z, t)-(x, y)((\bar{x},-y)(z, t)) \\
& =\underbrace{(q(x) z-\alpha q(y) z, q(x) t-\alpha q(y) t)}_{(1)}-\underbrace{(x, y)(\bar{x} z-\alpha t \bar{y}, x t-z y)}_{(2)}
\end{aligned}
$$

Desenvolvendo a parcela (2), temos:

$$
\begin{aligned}
(2) & =(x(\bar{x} z-\alpha t \bar{y})+\alpha(x t-z t) \bar{y}, \bar{x}(x t-z y)+(\bar{x} z-\alpha t \bar{y}) y) \\
& =(x(\bar{x} z)-\alpha x(t \bar{y})+\alpha(x t) \bar{y}-\alpha(z y) \bar{y}, \bar{x}(x t)-\bar{x}(z y)+(\bar{x} z) y-\alpha(t \bar{y}) y)
\end{aligned}
$$

Notando que: $x(\bar{x} z)=q(x) z ; \quad(z y) \bar{y}=q(y) z ; \quad \bar{x}(x t)=q(x) t ; \quad(t \bar{y}) y=q(y) t$ a expressão (2) fica: 
$(2)=(q(x) z-\alpha q(y) z-\alpha x(t \bar{y})+\alpha(x t) \bar{y}, q(x) t-\alpha q(y) t-\bar{x}(z y)+(\bar{x} z) y)$

Finalmente, fazendo $(1)-(2)$ obtemos: $(X, \bar{X}, Z)=(-\alpha(x, t, \bar{y}),-(\bar{x}, z, y))$.

Chegamos assim a uma expressão que relaciona o associador em $(A, \alpha)$ com o associador em $A$. Concluímos diretamente da expressão obtida que $(A, \alpha)$ é alternativa se e somente se $A$ é associativa.

Cabem ainda algumas investigações na álgebra $(A, \alpha)$. Os próximos dois teoremas nos trazem mais esclarecimentos.

Teorema 3.3.4. A álgebra $(A, \alpha)$ é associativa se e somente se $A$ é comutativa.

Demonstração: Sejam $X=(x, y), U=(u, v)$ e $Z=(z, t) \in(A, \alpha)$. Calculemos:

$$
\begin{aligned}
&(X, U, Z)=(X U) Z-X(U Z)=\underbrace{((x, y)(u, v))(z, t)}_{(1)}-\underbrace{(x, y)((u, v)(z, t))}_{(2)} \\
&(1)=(x y+\alpha v \bar{y}, \bar{x} v+u y)(z, t) \\
&=(x y+\alpha v \bar{y}) z+\alpha t(\overline{(\bar{x} v+u y)}, \overline{(x y+\alpha v \bar{y})}+z(\bar{x} v+u y)) \\
&=(x y) z+\alpha(v \bar{y}) z+\alpha t(\overline{\bar{x} v}+\overline{u y}),(\overline{x y}+\alpha \bar{v} \bar{y}) t+z(\bar{x} v)+z(u y)) \\
&=(x y) z+\alpha(v \bar{y}) z+\alpha t(\bar{v} x+\overline{y u}),(\overline{y x}+\alpha y \bar{v}) t+z(\bar{x} v)+z(u y)) \\
&=(x y) z+\alpha(v \bar{y}) z+\alpha t(\bar{v} x)+\alpha t(\overline{y u}),(\overline{y x}) t+\alpha(y \bar{v}) t+z(\bar{x} v)+z(u y)) \\
&(2)=(x, y)(u z+\alpha t \bar{v}, \bar{u} t+z v) \\
&=(x(u z+\alpha t \bar{v})+\alpha(\bar{u} t+z v) \bar{y}, \bar{x}(\bar{u} t+z v)+(u z+\alpha t \bar{v}) y) \\
&=(x(u z)+\alpha x(t \bar{v})+\alpha(\bar{u} t) \bar{y}+\alpha(z v) \bar{y}, \bar{x}(\bar{u} t)+\bar{x}(z v)+(u z) y+\alpha(t \bar{v}) y)
\end{aligned}
$$

Fazendo $x=u=t=0$ e $y=1$, obtemos:

$$
(X, U, Z)=(1)-(2)=\alpha(v z)-\alpha(z v)
$$

Como $\alpha \neq 0$, a expressão acima nos diz que $(A, \alpha)$ é associativa se e somente se $A$ é comutativa.

Teorema 3.3.5. A álgebra $(A, \alpha)$ obtida de A pelo processo de duplicação de CayleyDickson é comutativa se e somente se A é comutativa e $j=1$ (involução identidade).

Demonstração: Sejam $X=(x, y)$ e $U=(u, v) \in(A, \alpha)$. Calculemos:

$$
[X, U]=X U-U X=(x, y)(u, v)-(u, v)(x, y)
$$




$$
\begin{aligned}
& =(x u+\alpha v \bar{y}, \bar{x} v+u y)-(u x+\alpha y \bar{v}, \bar{u} y+x v) \\
& =(x u-u x+\alpha(v \bar{y}-y \bar{v}),(\bar{x}-x) v+(u-\bar{u}) y)
\end{aligned}
$$

Vemos assim que, para que $(A, \alpha)$ seja comutativa, isto é $[X, U]=0, A$ deve ser comutativa e $x=\bar{x}$, para todos $x \in A$, o que significa que a involução $j$ deve ser a identidade.

\subsection{Exemplos de Álgebras de Composição}

O exemplo mais trivial de álgebra de composição é o próprio corpo $F$. De fato, em $F$ definimos $q(x)=x^{2}$, donde $q(x y)=(x y)^{2}=x^{2} y^{2}=q(x) q(y)$, que é claramente não degenerada. Temos também que $q(x)=x^{2}=x \bar{x} \Rightarrow \bar{x}=x, \log j=1$. Tomando $\alpha \neq 0$ em $F$ construímos a álgebra $(F, \alpha)$ de dimensão 2 que é, pelo que vimos anteriormente, comutativa e associativa.

Tomando outro elemento não nulo em $F$, digamos $\beta \in F$, duplicamos $(F, \alpha)$, obtendo $((F, \alpha), \beta)$, que são álgebras de dimensão 4 chamadas álgebras dos quatérnios generalizados, que se costuma designar por $Q$. Assim $Q(\alpha, \beta)=((F, \alpha), \beta)$.

Quanto à involução em $(F, \alpha)$ temos, pela expressão geral $j:(F, \alpha) \rightarrow(F, \alpha)$ é dada por $j(x, y)=\overline{(x, y)}=(\bar{x},-y)$. Assim, como a involução $j$ não é o operador identidade, a álgebra $Q(\alpha, \beta)$ dos quatérnios generalizados não é comutativa. Mas, sendo $(F, \alpha)$ comutativa, $Q(\alpha, \beta)$ é associativa.

Continuando o processo, ao duplicarmos $Q$, obteremos uma álgebra alternativa, mas não associativa. Tomando então $\gamma \in F, \gamma \neq 0$, obtemos a álgebra $(Q(\alpha, \beta), \gamma)$ de dimensão 8, que denotamos por $C(\alpha, \beta, \gamma)$ ou, simplesmente $C$. Como $C$ é alternativa, é também uma álgebra de composição. Tal álgebra é chamada álgebra de CayleyDickson ou álgebra dos octônios.

Se continuarmos o processo, duplicando agora a álgebra $C$ obteremos uma álgebra que não é alternativa (pois $C$ não é associativa) e, portanto, não é de composição. Desta forma, partindo do corpo $F$ obtemos apenas 4 álgebras de composição: o próprio corpo $F$, de dimensão 1 , as álgebras quadráticas, de dimensão 2 , as álgebras dos quatérnios, de dimensão 4 e as álgebras dos octônios, de dimensão 8.

Mostraremos a seguir que estas são as únicas álgebras de composição, a menos de isomorfismos. Para isto consideraremos uma álgebra de composição $A$ arbitrária e $B$ 
subálgebra de $A$ satisfazendo certas condições. Então $B$ pode ser duplicada através do processo de Cayley-Dickson, obtendo uma álgebra $C$, subálgebra de $A$ satisfazendo as mesmas condições de $B$.

Definição 3.4.1. Sejam A um espaço vetorial em que está definida uma forma bilinear não degenerada $f(x, y)$ e $B$ subespaço próprio de A. Chama-se complemento ortogonal de $B$ em relação a $f(x, y)$ ao conjunto

$$
B^{\perp}=\{a \in A \mid f(a, b)=0, \forall b \in B\} .
$$

Lema 3.4.1. A forma bilinear $f(x, y)$ é não degenerada em $B^{\perp}$.

Demonstração: Seja $\left.f\right|_{B^{\perp}}(a, b)=0$, para todo $b \in B^{\perp}$. Então

$$
a \in\left(B^{\perp}\right)^{\perp}=B \Rightarrow a \in B^{\perp} \cap B \Rightarrow a=0
$$

Lema 3.4.2. Seja A uma álgebra de composição e B subálgebra de A contendo 1, tal que a restrição de $f(x, y)$ a $B$ é não degenerada. Valem os seguintes resultados:

(i) $\bar{e}=-e, \quad$ para todo $e \in B^{\perp}$;

(ii) $x e=e \bar{x}, \quad$ para todo $e \in B^{\perp}$ e todo $x \in B$;

(iii) $x(e y)=e(\bar{x} y)$;

(iv) $(e y) x=e(x y)$;

(v) $(e x)(e y)=\alpha y \bar{x}$, onde $\alpha=-q(e)$;

(vi) Seja e $\in B^{\perp}$ tal que $q(e) \neq 0$; Então $B_{1}=B \oplus e B$ é a subálgebra de A obtida de $B$ pelo processo de Cayley-Dickson.

\section{Demonstração:}

(i) Para todo $e \in B^{\perp}, f(e, y)=0, \forall y \in B$. Em particular, $f(e, 1)=0 \Rightarrow \bar{e}+e=0 \Rightarrow \bar{e}=-e$

(ii) $f(x, e)=x \bar{e}+e \bar{x}=0 \Rightarrow x \bar{e}=-e \bar{x} \Rightarrow-x e=-e \bar{x} \Rightarrow x e=e \bar{x}$ 
(iii) Pelo Lema 3.2.4: $x(\bar{x} y)=(x \bar{x}) y=q(x) y$. Linearizando em relação a $x$, temos:

$$
\begin{aligned}
& (x+z)(\overline{(x+z)} y)=q(x+z) y \Rightarrow(x+z)(\bar{x} y+\bar{z} y)=q(x+z) y \\
& \Rightarrow x(\bar{x} y)+x(\bar{z} y)+z(\bar{x} y)+z(\bar{z} y)=(x+z)(\bar{x}+\bar{z}) y \\
& \Rightarrow x(\bar{x} y)+x(\bar{z} y)+z(\bar{x} y)+z(\bar{z} y)=(x \bar{x}) y+(x \bar{z}) y+(z \bar{x}) y+(z \bar{z}) y \\
& \Rightarrow x(\bar{z} y)+z(\bar{x} y)=(x \bar{z}+z \bar{x}) y \Rightarrow x(\bar{z} y)+z(\bar{x} y)=f(x, z) y
\end{aligned}
$$

Substituindo $z$ por $e$ :

$$
x(\bar{e} y)+e(\bar{x} y)=0 \Rightarrow x(\bar{e} y)=-e(\bar{x} y) \stackrel{(i)}{\Rightarrow}-x(e y)=e(\bar{x} y) \Rightarrow x(e y)=e(\bar{x} y)
$$

(iv) Podemos escrever (iii) como $\bar{x}(e y)=e(x y)$. Aplicando involução e usando (ii):

$$
\begin{aligned}
& \overline{\bar{x}(e y)}=\overline{e(x y)} \Rightarrow \overline{(e y)} x=\overline{(x y)} \bar{e} \Rightarrow(\bar{y} \bar{e}) x=-\overline{(x y)} e \stackrel{(i i)}{\Rightarrow}-(\bar{y} e) x=-e(x y) \\
& \Rightarrow(e y) x=e(x y)
\end{aligned}
$$

$(\mathrm{v})(e x)(e y) \stackrel{(i i)}{=}(e x)(\bar{y} e) \stackrel{*}{=} e(x \bar{y}) e \stackrel{* *}{=}((y \bar{x}) e) e=y(\bar{x}) e^{2}=(y \bar{x})(e(-\bar{e}))=-(y \bar{x})(e \bar{e})$

$$
\begin{aligned}
& =-(y \bar{x}) q(e)=\alpha y \bar{x} \\
& \left({ }^{*}\right) \text { Moufang } \quad(* *) e(x \bar{y})=(\overline{x \bar{y}}) e=(y \bar{x}) e
\end{aligned}
$$

(vi) Calculemos, para $x, y, z, t \in B$ :

$$
\begin{aligned}
(x+e y)(z+e t) & =x z+x(e t)+(e y) z+(e y)(e t)=x z+e(\bar{x} t)+e(z y)+\alpha t \bar{y} \\
& =x z+\alpha t \bar{y}+e(\bar{x} t+z y)
\end{aligned}
$$

Portanto $(x+e y)(z+e t) \in B_{1}=B \oplus e B$. Logo, $B_{1}$ é subálgebra de $A$.

O produto em $B_{1}$ é o mesmo dado pela duplicação de $B$ por Cayley-Dickson, conforme verificamos a seguir:

$$
(x, y)(z, t)=(x z+\alpha t \bar{y}, \bar{x} t+z y)=x z+\alpha t \bar{y}+e(\bar{x} t+z y)
$$

Falta ainda mostrar que:

(a) A involução em $B \oplus e B$ é a mesma em $(B, \alpha)$

Aplicando (i) e (ii): $\overline{x+e y}=\bar{x}+\overline{e y}=\bar{x}+\bar{y} \bar{e}=\bar{x}-\bar{y} e=\bar{x}-e y$ 
(b) $B$ e $e B$ têm a mesma dimensão

Pelo Lema 3.2.2 (ii) tem-se: $q(x) f(y, w)=f(x y, x w)$, para todos $x, y, w \in A$. Fazendo $x=e, y=a$ e $w=b$, obtemos: $f(e a, e b)=q(e) f(a, b)=-\alpha f(a, b)$.

Então, a aplicação $\varphi: B \rightarrow e B$ dada por $\varphi(b)=e b$ é injetora. De fato, $x \in$ $\operatorname{ker}(\varphi) \Rightarrow \varphi(x)=e x=0$. Como $f$ é não degenerada, devemos ter: $f(e x, e y)=$ $f(0, e y)=-\alpha f(x, y)=0 \Rightarrow f(x, y)=0 \Rightarrow x=0$. Como $\varphi$ é claramente sobrejetora, temos uma bijeção de $B$ em $e B$. Logo $B$ e $e B$ têm a mesma dimensão.

(c) A forma $f(x, y)$ restrita a $B \oplus e B$ é não degenerada

Mostremos inicialmente que $e x \in B^{\perp}$, para todo $x \in B$. Basta mostrar que $f(b, e x)=0$, para todo $b \in B$. Pelo Lema 3.2.2 (iii):

$f(b, e x)=f(b .1, e x)=f(b, e) f(1, x)-f(b x, e)=0 f(1, x)-0=0$.

Seja $f(x+e y, z+e t)=0, \forall z+e t \in B \oplus e B$. Então:

$0=f(x, z)+\underbrace{f(x, e t)}_{=0}+\underbrace{f(e y, z)}_{=0}+\underbrace{f(e y, e t)}_{=0} \Rightarrow f(x, z)=0 \Rightarrow x=0$.

Tem-se ainda: $f(e y, e t)=-\alpha f(y, t)=0 \stackrel{\alpha \neq 0}{\Rightarrow} f(y, t)=0 \Rightarrow y=0$.

Portanto $x+e y=0$ e $f$ é não degenerada em $B \oplus e B$.

Mostramos assim que $B \oplus e B$ é a subálgebra de $A$ obtida de $B$ pelo processo de Cayley-Dickson. Podemos escrever então: $B_{1}=(B, \alpha)=B \oplus e B$.

Pelo que foi mostrado, $(B, \alpha)$ satisfaz as mesmas condições de $B$ e podemos aplicar o processo de Cayley-Dickson em $(B, \alpha)$. Veremos no próximo teorema que as álgebras de composição se restringem aos quatro exemplos apresentados.

Teorema 3.4.3 (Hurwitz). As únicas álgebras de composição sobre um corpo $F$ de característica $\neq 2$ são:

(i) $F$;

(ii) As álgebras quadráticas $(F, \alpha)$;

(iii) As álgebras de quatérnios; 
(iv) As álgebras de Cayley-Dickson.

Demonstração: As álgebras citadas são de composição conforme já sabemos. Para mostrar que estas são as únicas, consideremos uma álgebra de composição $A$. Se $A=F$ então temos o caso (i). Se $A \neq F$, tomamos $B=F$, subálgebra de $A$ contendo 1 e, como $f$ é não degenerada em $F$ podemos aplicar Cayley-Dickson em $F$ obtendo $(F, \alpha) \subseteq A$. Se $A=(F, \alpha)$ obtemos (ii). Se $A \neq(F, \alpha)$, fazemos $B=(F, \alpha)$, podemos duplicar $B$ por Cayley-Dickson, pois, pelo Lema 3.4.1, $f$ é não degenerada em $B$. Obtemos a álgebra $Q$ dos quatérnios, $Q \subseteq A$. Se $A=Q$ o item (iii) está demonstrado. Se $A \neq Q$, duplicamos $Q$ ( $f$ é não degenerada em $Q$ ), obtendo uma álgebra de CayleyDickson $C$ contida em $A$. Se $A=C$ provamos (iv). Se $A \neq C$, podemos duplicar $C$ por Cayley-Dickson obtendo uma álgebra $D$ que não é de composição, pois $C$ não é associativa. Mas isto é impossível, pois $D \subseteq A$ e $A$ é de composição. Devemos ter então, necessariamente $A=C$ e o teorema está provado.

\subsection{Hurwitz e a Soma de Quadrados}

Vamos agora fazer uma relação das álgebras de composição com o problema da soma de quadrados. Para isto precisaremos construir a tábua de multiplicação da álgebra de Cayley, $C$. Definiremos os produtos para esta tábua a partir dos elementos de uma base de $C$. Como é uma base de $C$ visto como um espaço vetorial de dimensão 8? Vamos fazer esta construção passo a passo, começando com o corpo $F$, definindo as diversas multiplicações em cada uma das álgebras de composição.

A primeira álgebra de composição é o próprio corpo $F$, cuja base é $\{1\}$, a unidade de $F$. Vamos designar $e_{0}=1$. Para um dado $\alpha \in F, \alpha \neq 0$, obtemos $(F, \alpha)$ na qual um elemento se escreve $x .1+e_{1} y$ onde $e_{1}=(0,1)$ e $e_{1}^{2}=\alpha$. São as álgebras quadráticas sobre $F$, que vamos denotar por $F \oplus e_{1} F$. Uma base de $(F, \alpha)$ é $\left\{1, e_{1}\right\}$. Como $F$ é corpo, podemos fazer a multiplicação por $e_{1}$ à esquerda, como usualmente se procede para escrever combinações lineares em um espaço vetorial. Assim, se $X=(x, y) \in(F, \alpha)$, temos: $X=x+y e_{1}$ e daí $(F, \alpha)=F \oplus F e_{1}$. A tábua de multiplicação em $(F, \alpha)$ é dada a seguir:

\begin{tabular}{|c||c|c|}
\hline & 1 & $e_{1}$ \\
\hline \hline 1 & 1 & $e_{1}$ \\
\hline$e_{1}$ & $e_{1}$ & $\alpha$ \\
\hline
\end{tabular}


Tomamos agora $\beta \in F, \beta \neq 0$, e construímos a álgebra dos quatérnios $Q$ designada por $((F, \alpha), \beta)$. Um elemento de $Q$ se escreve $X+e_{2} Z$, com $X, Z \in(F, \alpha), X=$ $(x, y), Z=(z, t)$ e $e_{2}^{2}=\beta$. Desta forma escrevemos $Q=(F, \alpha) \oplus e_{2}(F, \alpha)$. Assim como fizemos nas álgebras quadráticas gostaríamos de multiplicar por $e_{2}$ à esquerda, para podermos formalizar uma base em $Q$. Notando que $e_{2} \in Q^{\perp}$, o Lema 3.4.2 (i),(ii) permite fazer esta alteração. Escrevemos, então: $Q=(F, \alpha) \oplus(F, \alpha) e_{2}$. Assim, um elemento de $Q$ é dado por:

$$
X+Z e_{2}=x+y e_{1}+\left(z+t e_{1}\right) e_{2}=x+y e_{1}+z e_{2}+\left(t e_{1}\right) e_{2}
$$

Como $Q$ é associativa, $\left(t e_{1}\right) e_{2}=t\left(e_{1} e_{2}\right)$. Logo:

$$
X+Z e_{2}=x+y e_{1}+z e_{2}+t\left(e_{1} e_{2}\right)
$$

Temos então que $\left\{1, e_{1}, e_{2}, e_{1} e_{2}\right\}$ é uma base de $Q$. Ou, simplificando a notação, fazemos $e_{1} e_{2}=e_{3}$ e daí, a base de $Q$ será: $\left\{1, e_{1}, e_{2}, e_{3}\right\}$. A tábua de multiplicação em $Q$ é indicada abaixo:

\begin{tabular}{|c||c|c|c|c|}
\hline & 1 & $e_{1}$ & $e_{2}$ & $e_{3}$ \\
\hline \hline 1 & 1 & $e_{1}$ & $e_{2}$ & $e_{3}$ \\
\hline$e_{1}$ & $e_{1}$ & $\alpha$ & $e_{3}$ & $\alpha e_{2}$ \\
\hline$e_{2}$ & $e_{2}$ & $-e_{3}$ & $\beta$ & $-\beta e_{1}$ \\
\hline$e_{3}$ & $e_{3}$ & $-\alpha e_{2}$ & $\beta e_{1}$ & $-\alpha \beta$ \\
\hline
\end{tabular}

Destacamos alguns cálculos realizados para se chegar a estes valores. Lembrando que, pela regra de multiplicação estabelecida por Hamilton, temos:

$$
e_{1} e_{2}=-e_{2} e_{1} \quad e_{1} e_{3}=-e_{3} e_{1} \quad e_{2} e_{3}=-e_{3} e_{2}
$$

Calculamos: $\quad e_{1} e_{3}=e_{1}\left(e_{1} e_{2}\right)=e_{1}^{2} e_{2}=\alpha e_{2}$

$$
\begin{aligned}
& e_{3} e_{2}=\left(e_{1} e_{2}\right) e_{2}=e_{1} e_{2}^{2}=\beta e_{1} \\
& e_{3}^{2}=\left(e_{1} e_{2}\right)^{2}=\left(e_{1} e_{2}\right)\left(e_{1} e_{2}\right)=-\left(e_{2} e_{1}\right)\left(e_{1} e_{2}\right)=-\left(\left(e_{2} e_{1}\right) e_{1}\right) e_{2} \\
& \quad=-\left(e_{2} e_{1}^{2}\right) e_{2}=-\left(\alpha e_{2}\right) e_{2}=-\alpha e_{2}^{2}=-\alpha \beta
\end{aligned}
$$

Vamos agora à construção da álgebra dos octônios. Tomamos $\gamma \in F, \gamma \neq 0$ e obtemos a álgebra $C=(Q(\alpha, \beta), \gamma)$, que é formada por pares de elementos de $Q(\alpha, \beta)$. Escrevemos então $C=(Q(\alpha, \beta), \gamma)=Q(\alpha, \beta) \oplus Q(\alpha, \beta) e_{4}$, onde $e_{4}^{2}=\gamma$. Assim, um elemento de $C$ se escreve: $u+v e_{4}$, com $u, v \in Q(\alpha, \beta)$. Sejam $u=a+b e_{1}+c e_{2}+d e_{3}$ e $v=e+f e_{1}+g e_{2}+h e_{3}$. Daí: 


$$
\begin{aligned}
u+v e_{4} & =\left(a+b e_{1}+c e_{2}+d e_{3}\right)+\left(e+f e_{1}+g e_{2}+h e_{3}\right) e_{4} \\
& =a+b e_{1}+c e_{2}+d e_{3}+e e_{4}+f\left(e_{1} e_{4}\right)+g\left(e_{2} e_{4}\right)+h\left(e_{3} e_{4}\right)
\end{aligned}
$$

Fazemos: $e_{1} e_{4}=e_{5}, e_{2} e_{4}=e_{6}, e_{3} e_{4}=e_{7}$ e obtemos uma base de $C$ :

$$
\left\{1, e_{1}, e_{2}, e_{3}, e_{4}, e_{5}, e_{6}, e_{7}\right\} \text {. }
$$

A tábua de multiplicação de $C$ é dada abaixo:

\begin{tabular}{|c||c|c|c|c|c|c|c|c|}
\hline & 1 & $e_{1}$ & $e_{2}$ & $e_{3}$ & $e_{4}$ & $e_{5}$ & $e_{6}$ & $e_{7}$ \\
\hline \hline 1 & 1 & $e_{1}$ & $e_{2}$ & $e_{3}$ & $e_{4}$ & $e_{5}$ & $e_{6}$ & $e_{7}$ \\
\hline$e_{1}$ & $e_{1}$ & $\alpha$ & $e_{3}$ & $\alpha e_{2}$ & $e_{5}$ & $\alpha e_{4}$ & $-e_{7}$ & $-\alpha e_{6}$ \\
\hline$e_{2}$ & $e_{2}$ & $-e_{3}$ & $\beta$ & $-\beta e_{1}$ & $e_{6}$ & $e_{7}$ & $\beta e_{4}$ & $\beta e_{5}$ \\
\hline$e_{3}$ & $e_{3}$ & $-\alpha e_{2}$ & $\beta e_{1}$ & $-\alpha \beta$ & $e_{7}$ & $\alpha e_{6}$ & $-\beta e_{5}$ & $-\alpha \beta e_{4}$ \\
\hline$e_{4}$ & $e_{4}$ & $-e_{5}$ & $-e_{6}$ & $-e_{7}$ & $\gamma$ & $-\gamma e_{1}$ & $-\gamma e_{2}$ & $-\gamma e_{3}$ \\
\hline$e_{5}$ & $e_{5}$ & $-\alpha e_{4}$ & $-e_{7}$ & $-\alpha e_{6}$ & $\gamma e_{1}$ & $-\alpha \gamma$ & $\gamma e_{3}$ & $\alpha \gamma e_{2}$ \\
\hline$e_{6}$ & $e_{6}$ & $e_{7}$ & $-\beta e_{4}$ & $\beta e_{5}$ & $\gamma e_{2}$ & $-\gamma e_{3}$ & $-\beta \gamma$ & $-\beta \gamma e_{1}$ \\
\hline$e_{7}$ & $e_{7}$ & $\alpha e_{6}$ & $-\beta e_{5}$ & $\alpha \beta e_{4}$ & $\gamma e_{3}$ & $-\alpha \gamma e_{2}$ & $\beta \gamma e_{1}$ & $\alpha \beta \gamma$ \\
\hline
\end{tabular}

Indicamos a seguir o cálculo de alguns dos elementos da tabela:

$$
\begin{aligned}
& e_{1} e_{6}=e_{1}\left(e_{2} e_{4}\right) \stackrel{*}{=} e_{1}\left(e_{4} \bar{e}_{2}\right) \stackrel{* *}{=} e_{4}\left(\bar{e}_{1} \bar{e}_{2}\right) \stackrel{* * *}{=} e_{4}\left(e_{1} e_{2}\right)=e_{4} e_{3}=-e_{7} \\
& \left.\quad{ }^{*}\right) \text { Lema 3.4.2(ii) } \quad\left({ }^{* *}\right) \text { Lema 3.4.2(iii) } \quad(* * *) \text { Lema 3.4.2 (i) } \\
& e_{5}^{2}=\left(e_{1} e_{4}\right)\left(e_{1} e_{4}\right)=\left(e_{1} e_{4}\right)\left(e_{4} \bar{e}_{1}\right)=e_{4}\left(\left(\bar{e}_{1} e_{4}\right) \bar{e}_{1}\right)=e_{4}\left(\left(\bar{e}_{4} \bar{e}_{1}\right) \bar{e}_{1}\right)=e_{4}\left(\bar{e}_{4} e_{1}^{2}\right) \\
& =-\alpha e_{4}^{2}=-\alpha \gamma \\
& e_{6} e_{7}=\left(e_{2} e_{4}\right)\left(e_{3} e_{4}\right) \stackrel{*}{=} e_{2}\left(\left(e_{3} e_{4}\right) e_{4}\right)=e_{2}\left(e_{3} e_{4}^{2}\right)=\gamma e_{2} e_{3}=-\beta \gamma e_{1}
\end{aligned}
$$

(*) Fazendo $e=e_{2}, y=e_{4}$ e $x=\left(e_{3} e_{4}\right)$ no Lema 3.4 .2 (iv)

Evidentemente existem outras maneiras de se efetuar estes cálculos.

Vamos agora relacionar o problema de Hurwitz (Teorema 3.4.3) com o correspondente problema numérico, a que nos referimos como a "soma de quadrados" no início deste capítulo. Tal relação é possível devido à propriedade da forma quadrática $q$ de admitir composição, isto é, $q(x y)=q(x) q(y)$. Ilustraremos esta relação com a álgebra dos quatérnios, uma vez que, para as outras álgebras de composição, o procedimento é idêntico, sendo bastante elementar nos casos do corpo $F$ e das quadráticas $(F, \alpha)$ e um tanto trabalhoso para os octônios. 
Consideremos então o produto de dois elementos dos quatérnios, a que chamaremos $X$ e $Y$, por simplicidade de notação. Façamos $X=a+b e_{1}+c e_{2}+d e_{3}$ e $Y=e+f e_{1}+g e_{2}+h e_{3}$.

$$
\text { Calculemos } \begin{aligned}
X Y & =\left(a+b e_{1}+c e_{2}+d e_{3}\right)\left(e+f e_{1}+g e_{2}+h e_{3}\right) \\
& =(a e+\alpha b f+\beta c g-\alpha \beta d h)+(a f+b e-\beta c h+\beta d g) e_{1} \\
& +(a g+c e+\alpha b h-\alpha d f) e_{2}+(a h+d e+b g-c f) e_{3}
\end{aligned}
$$

Pelo que já vimos até agora, resulta imediato que a involução em $Q$ é dada por:

$$
\bar{X}=a-b e_{1}-c e_{2}-d e_{3}
$$

Calculando $\quad q(X)=X \bar{X}=\left(a+b e_{1}+c e_{2}+d e_{3}\right)\left(a-b e_{1}-c e_{2}-d e_{3}\right)$ encontramos $q(X)=a^{2}-\alpha b^{2}-\beta c^{2}+\alpha \beta d^{2}$.

De maneira análoga, obtemos para $Y=e+f e_{1}+g e_{2}+h e_{3}$

$$
q(Y)=e^{2}-\alpha f^{2}-\beta g^{2}+\alpha \beta h^{2}
$$

Da expressão de $X Y$ deduzida anteriormente, temos:

$$
\begin{aligned}
q(X Y) & =(a e+\alpha b f+\beta c g-\alpha \beta d h)^{2}-\alpha(a f+b e-\beta c h+\beta d g)^{2} \\
& -\beta(a g+c e+\alpha b h-\alpha d f)^{2}+\alpha \beta(a h+d e+b g-c f)^{2}
\end{aligned}
$$

Se $F=\mathbb{R}$ e $\alpha=\beta=-1$ temos a expressão do produto de uma soma de 4 quadrados é também uma soma de 4 quadrados:

$$
\begin{aligned}
\left(a^{2}+b^{2}+c^{2}+d^{2}\right)\left(e^{2}+f^{2}+g^{2}+h^{2}\right) & =(a e-b f-c g-d h)^{2}+(a f+b e+c h-d g)^{2} \\
& +(a g+c e-b h+d f)^{2}+(a h+d e+b g-c f)^{2} .
\end{aligned}
$$

Ainda sobre o Teorema de Hurwitz, é importante destacar as álgebras de composição obtidas fazendo $F=\mathbb{R}$, e os escalares $\alpha=\beta=\gamma=-1$. Ao duplicarmos $\mathbb{R}$ pelo processo de Cayley-Dickson obtemos a Álgebra dos Complexos, $\mathbb{C}=(\mathbb{R},-1)$. Duplicando $\mathbb{C}$ obtemos os Quatérnios de Hamilton, $\mathbb{H}=(\mathbb{C},-1)$. Finalmente, a partir de $\mathbb{H}$ chegamos aos Octônios, $\mathbb{O}=(\mathbb{H},-1)$. São estes casos particulares de álgebras de composição, mais especificamente os dois últimos, que serão estudados no próximo capítulo. 


\section{Capítulo 4}

\section{Identidades em Álgebras de Cayley}

Faremos um breve estudo das identidades de graus 4 e 5 nas álgebras de Cayley, de acordo com algumas das mais recentes pesquisas realizadas sobre o assunto. Nossa abordagem se apóia no artigo Minimal Identities of Octonion Algebras, de M. L. Racine (ref. [19]), onde é provado que não existem identidades de grau 4 nas álgebras de octônios e, que as identidades de grau 5 resultam de outras duas identidades conhecidas.

Mencionamos também alguns resultados obtidos por outros estudiosos do assunto sobre identidades de grau 6 ou maior, sempre em um corpo de característica 0 ou maior que o grau da identidade em estudo.

\subsection{Introdução}

Para uma primeira noção do que é uma identidade, consideremos um corpo $F$, uma $F$-álgebra $A$ e um polinômio $f\left(x_{1}, \ldots, x_{n}\right)$ nas indeterminadas não comutativas e não associativas $x_{1}, \ldots x_{n}$ e com coeficientes em $F$. Dizemos que $f$ é uma identidade de $A$ se $f\left(a_{1}, \ldots, a_{n}\right)=0$ para todos $a_{1}, \ldots, a_{n} \in A$. Dizemos também que $A$ satisfaz $f$. Assim, uma álgebra comutativa satisfaz a identidade $x y-y x$ e, uma álgebra associativa satisfaz a identidade $(x y) z-x(y z)$. Daremos adiante uma definição mais formal, abrangendo outros conceitos importantes para as considerações aqui apresentadas.

De fundamental importância para o estudo das identidades são as álgebras de matrizes $M_{n}(F)$, ou mais genericamente, $M_{n}(R)$, onde $R$ é um anel comutativo. De que forma se relacionam matrizes e identidades? Para as identidades polinomiais pode-se definir o seu grau. Veremos em breve que uma identidade de grau $t$ satis- 
fazendo certas condições é múltipla do polinômio standard do grau $t$, denotado por $s_{t}$, que será definido oportunamente. Escrevemos: $f=\alpha s_{t}$. Por outro lado, o Teorema de Amitsur-Levitzki, que daremos uma demonstração adiante, afirma que $s_{2 n}$ é uma identidade do anel das matrizes de ordem $n$ com coeficientes em $R$, que designamos por $M_{n}(R)$.

Vamos agora formalizar estes resultados para podermos abordar as identidades de grau 4 e 5 nas álgebras de Cayley.

\section{2 Álgebras Livres}

Seja $X$ um conjunto arbitrário, podendo ser infinito. Designamos por $V[X]$ o conjunto obtido a partir de $X$ das palavras não associativas e não comutativas, formado pelos elementos de $X$ mais os parêntesis à esquerda e à direita.

Os elementos de $V[X]$ são agrupamentos convenientes dos elementos de $X$, de tal forma que se $x_{1}, x_{2}, x_{3} \in X$, o agrupamento $x_{1} x_{2} \in V[X]$, mas $x_{1} x_{2} x_{3} \notin V[X]$. Para agrupar mais de dois elementos devemos usar os parêntesis. Assim, $\left(x_{1} x_{2}\right) x_{3} \in V[X]$ e $x_{2}\left(x_{3} x_{1}\right) \in V[X]$. É claro que $x_{1}, x_{2} \in V[X]$ e mais ainda, $X \subseteq V[X]$.

Sejam $u, v \in V[X] \backslash X$. Se $x_{1}, x_{2} \in X$, temos que $x_{1} x_{2}, x_{1}(u),(v) x_{2},(u)(v) \in V[X]$. Podemos definir em $V[X]$ uma multiplicação. Se $x_{1}, x_{2}, u, v \in V[X]$, com $x_{1}, x_{2} \in X$, definimos:

$$
\begin{array}{ll}
x_{1} \cdot x_{2}=x_{1} x_{2} ; & x_{1} \cdot u=x_{1}(u) \\
v \cdot x_{2}=(v) x_{2} ; & u \cdot v=(u)(v)
\end{array}
$$

Então, · é uma operação binária em $V[X]$, isto é, $x \cdot y \in V[X]$, para todos $x, y$ em $V[X]$. Seja agora um corpo $F$. O conjunto $V[X]$ pode ser considerado uma base do $F$-espaço vetorial $F[X]$, cujos elementos são da forma:

$$
\sum_{i} \alpha_{i} u_{i}, \quad \alpha_{i} \in F, u_{i} \in V[X]
$$

onde $\sum_{i} \alpha_{i} u_{i}$ indica uma soma finita.

Definimos uma multiplicação em $F[X]$ estendendo a multiplicação definida em $V[X]$, por:

$$
\left(\sum_{i} \alpha_{i} u_{i}\right)\left(\sum_{j} \beta_{j} v_{j}\right)=\sum_{i} \sum_{j} \alpha_{i} \beta_{j}\left(u_{i} \cdot v_{j}\right), \quad \alpha_{i}, \beta_{j} \in F, \quad u_{i}, v_{j} \in V[X]
$$


Obtemos assim, uma $F$-álgebra $F[X]$, que é chamada álgebra livre não associativa. Os elementos de $F[X]$ são chamados polinômios não associativos. Um elemento da forma $\alpha u$, com $\alpha \in F$ e $u \in V[X]$ é chamado um monômio não associativo.

O grau de um monômio é igual ao número de variáveis que nele comparecem, notando que, para todo $x$ em $F[X], x x=x^{2}$, neste caso contam-se duas variáveis. Citamos alguns exemplos:

$$
(u v) w \text { tem grau } 3 ; \quad\left(u^{2} v\right)(w x) \text { tem grau } 5
$$

De maneira análoga, o grau de um polinômio é definido como o maior grau dos monômios que o formam. Assim, o polinômio $u^{2}+u v+(u v) w^{2}$ tem grau 4 .

Consideremos agora um conjunto enumerável $X=\left\{x_{1}, x_{2}, \ldots\right\}$. Seja $f \in F[X]$ arbitrário (sem o termo independente, se $A$ não tiver unidade), que é formado por uma quantidade finita de elementos de $X$. Indicamos por $f=f\left(x_{1}, x_{2}, \ldots, x_{n}\right)$. Seja $A$ uma $F$-álgebra e $a_{1}, a_{2}, \ldots, a_{n}$ em $A$. Ao substituirmos os $x_{i}$ de $f$ pelos $a_{i}$ obtemos o elemento $f\left(a_{1}, a_{2}, \ldots, a_{n}\right)$ em $A$. O polinômio não associativo e não comutativo $f$ é chamado uma identidade da álgebra $A$ se $f\left(a_{1}, a_{2}, \ldots, a_{n}\right)=0$, para todos $a_{1}, a_{2}, \cdots, a_{n}$ em $A$.

O conjunto de todas as identidades para uma dada álgebra $A$ é um ideal de $F[X]$, chamado ideal de identidades de $A$ ou $T$-ideal de $A$, denotado por $T(A)$.

Definição 4.2.1. Seja I um subconjunto de $F[X]$. A classe de todas as F-álgebras satisfazendo cada identidade de I é chamada variedade de álgebras definida pelo conjunto de identidades $I$.

Exemplos:

1. A variedade das $F$-álgebras associativas é definida pela identidade $f=(x y) z-x(y z)$;

2. A variedade das $F$-álgebras alternativas é definida pelas identidades $f_{1}=x^{2} y-x(x y)$ e $f_{2}=x y^{2}-(x y) y$, com $f_{1}$ definindo as álgebras alternativas à esquerda e $f_{2}$ definindo as álgebras alternativas à direita;

3. A variedade das álgebras de Lie é definida por $f_{1}=x^{2}$ e $f_{2}=(x y) z+(y z) x+(z x) y$ 
4. A variedade das álgebras de Jordan é definida por

$$
f_{1}=x y-y x \text { e } f_{2}=\left(x^{2} y\right) x-y^{2}(y x)
$$

Serão de importância para o nosso estudo, as variedades de álgebras dos exemplos 1 e 2, acima, nas quais estudaremos as álgebras associativas livres, designadas por $A s s[X]$ e as álgebras alternativas livres, designadas por $A l t[X]$, ambas sobre um conjunto de geradores $X$.

Dado um monômio nas variáveis $x_{1}, x_{2}, \ldots, x_{s}$, definimos o seu tipo como uma lista de números indicando o grau de cada uma das variáveis, designado por $\left[n_{1}, n_{2}, \ldots, n_{s}\right]$. Vejamos alguns exemplos:

1. $\left(x_{1}^{2} x_{2}\right)\left(x_{2} x_{3}^{2}\right)$ é do tipo $[2,2,2]$

2. $\left(\left(x_{1} x_{2}\right) x_{3}\right)\left(x_{3} x_{2}^{2}\right)$ é do tipo $[1,3,2]$

3. $\left(x_{1} x_{3}\right)\left(x_{4}\left(x_{1} x_{3}^{2}\right)\right)$ é do tipo $[2,0,3,1]$

Naturalmente, um polinômio $f$ pode ser escrito como soma de monômios.

Definição 4.2.2. Seja $f$ um polinômio nas variáveis $x_{1}, \ldots, x_{s}$, indicado por $f\left(x_{1}, \ldots, x_{s}\right)$. Dizemos que:

(i) $f$ é homogêneo em $x_{i}, 1 \leq i \leq s$, se o grau de $x_{i}$ for o mesmo em cada monômio de $f$;

(ii) $f$ é homogêneo, se for homogêneo em $x_{i}, \forall i=1, \ldots, s$;

(iii) $f$ é linear em $x_{i}, 1 \leq i \leq s$, se grau de $x_{i}=1$ em cada monômio de $f$;

(iv) $f$ é linear, se for linear em $x_{i}, \forall i=1, \ldots, s$;

(v) $f$ é t-linear, $1 \leq t \leq s$, se for linear em $x_{i}, \quad i=1, \ldots, t$;

(vi) $f$ é multilinear, se grau de $x_{i}=0$ ou grau de $x_{i}=1, \forall i=1, \ldots, s$;

(vii) $f$ é t-alternado, se $f\left(x_{1}, \ldots, x_{i}, \ldots, x_{j}, \ldots\right)=-f\left(x_{1}, \ldots, x_{j}, \ldots, x_{i}, \ldots\right)$ $\forall i, j, \quad 1 \leq i, j \leq t, \quad i \neq j ;$

(viii) $f$ é alternado, se for s-alternado; 
(ix) $f$ é t-normal, se for t-linear e t-alternado;

(x) f é normal, se for linear e alternado.

Os conceitos de grau, homogeneidade e polinômio multilinear homogêneo, conforme definidos acima, se aplicam à álgebra livre não associativa e não comutativa $F[X]$, sobre um conjunto gerador $X$. Para podermos aplicar estes conceitos nas álgebras associativas livres $A s s[X]$ e nas álgebras alternativas livres $A l t[X]$, precisamos identificar qual é o conjunto gerador $X$ para tais álgebras. Porém, não se conhece uma base natural em $\operatorname{Alt}[X]$.

Passamos então a considerar o ideal $I$ das identidades definidoras da variedade das álgebras associativas, que é o ideal gerado pelo conjunto

$$
I_{0}=\{(x, y, z) \mid x, y, z \in F[X]\}
$$

Podemos escrever ainda: $A s s[X]=F[X] / I$. Assim, cada elemento de $A s s[X]$, isto é, cada identidade polinomial associativa, é a imagem do homomorfismo canônico de um polinômio em $F[X]$, retirando-se os parêntesis. Designando este homomorfismo por $\phi: F[X] \rightarrow A s s[X]$, se $f \in F[X]$, então $\phi f$ é a imagem de $f$ por $\phi$ e $\phi f \in A s s[X]$.

De maneira análoga, seja $J$ o ideal das identidades definidoras das álgebras alternativas. Então, $J$ é o ideal gerado pelo conjunto

$$
J_{0}=\{(x, x, y) ;(z, t, t) \mid x, y, z, t \in F[X]\} .
$$

Assim, Alt $[X]=F[X] / J$ e, designando por $\nu: F[X] \rightarrow \operatorname{Alt}[X]$ o homomorfismo canônico entre estas duas álgebras, temos que, se $f \in F[X]$, então $\nu f \in A l t[X]$.

\subsection{Identidades Polinomiais}

Consideremos agora o grupo simétrico de ordem $t$, que denotaremos por $S_{t}$, também chamado de grupo de permutações, que é formado pelas permutações de $t$ elementos. Cada permutação será designada por $\pi$, em geral, afetada de um índice, como $\pi_{1}$ ou $\pi_{i_{1}}$. O grupo simétrico $S_{t}$ está intimamente relacionado com os polinômios multilineares associativos, uma vez que podemos facilmente verificar que se $f$ é um polinômio multilinear de grau $t$ em $A s s[X]$, então: 


$$
f=\sum_{\pi \in S_{t}} \alpha_{\pi} x_{\pi_{1}} \cdots x_{\pi_{t}}, \quad \operatorname{com} \alpha_{\pi} \in F
$$

Temos ainda que os polinômios multilineares associativos de grau $t$, designados por $P_{t}$, são isomorfos à álgebra de grupo $F S_{t}$, e que $P_{t}$ é um $S_{t}$-módulo.

Vejamos alguns exemplos:

1. O grupo simétrico do grau 2 é simplesmente formado pelas permutações (12) e (21). Assim, um polinômio multilinear do grau 2 poderá ser escrito:

$$
f\left(x_{1}, x_{2}\right)=\alpha x_{1} x_{2}+\beta x_{2} x_{1}, \quad \operatorname{com} \alpha, \beta \in F .
$$

2. Em grau 3, temos $3 !=6$ permutações de 3 elementos:

(123), (132), (213), (231), (312), (321). Então um polinômio multilinear do grau 3 terá a forma:

$f\left(x_{1}, x_{2}, x_{3}\right)=\alpha_{1} x_{1} x_{2} x_{3}+\alpha_{2} x_{1} x_{3} x_{2}+\cdots+\alpha_{6} x_{3} x_{2} x_{1}, \quad$ com $\alpha_{i} \in F$.

3. Um caso particular de polinômio multilinear de grau 3 é:

$$
f_{1}\left(x_{1}, x_{2}, x_{3}\right)=\alpha x_{1} x_{3} x_{2}+\beta x_{2} x_{3} x_{1}, \quad \alpha, \beta \in F
$$

Definição 4.3.1. O polinômio standard do grau $t$ é dado por:

$$
s_{t}\left(x_{1}, \ldots, x_{t}\right)=\sum_{\pi \in S_{t}}(\operatorname{sgn} \pi) x_{\pi_{1}} \cdots x_{\pi_{t}}
$$

onde $S_{t}$ é o grupo das permutações de $t$ elementos e $\operatorname{sgn} \pi$ indica o sinal da permutação.

Vê-se claramente que $s_{t}$ é um polinômio normal. Examinaremos a seguir alguns critérios que permitem estabelecer quando um polinômio linear é normal. No que segue, assumiremos que $f$ é um polinômio t-linear.

Definição 4.3.2. Para todas as permutações $\pi \in S_{k}, k \leq t$, definimos $f_{(k, \pi)}$ como a soma dos monômios nos quais as variáveis $x_{1}, \ldots, x_{k}$ aparecem na ordem $x_{\pi_{1}} \cdots x_{\pi_{k}}$ Se $\pi=1$ (permutação identidade) escrevemos $f_{(k)}$. Em particular, se $k=t$, obtemos: $f=\sum_{\pi \in S_{t}} f_{(t, \pi)}$.

Definição 4.3.3. Seja $f\left(x_{1}, \ldots, x_{s}\right)$ um polinômio t-linear. Definimos a composta $\pi \circ f, \pi \in S_{t}$, por:

$$
f\left(x_{\pi_{1}}, \ldots, x_{\pi_{t}}, x_{t+1}, \ldots, x_{s}\right)
$$


Para ilustrar um caso simples de uso da composta, podemos fazer $\pi=(12)$ e $f=x_{1} x_{3} x_{2}+x_{3} x_{2} x_{1}+x_{2} x_{1} x_{3}$. Então (12) ○ $f=x_{2} x_{3} x_{1}+x_{3} x_{1} x_{2}+x_{1} x_{2} x_{3}$.

Lema 4.3.1. Um polinômio t-linear $f$ é t-normal se e somente se $(i j) \circ f=-f$ $\forall i<j \leq t$.

Demonstração: $(\Rightarrow)$ Se $f$ é normal, então:

$(i j) \circ f+f=f\left(\ldots, x_{j}, \ldots, x_{i}, \ldots\right)+f\left(\ldots, x_{i}, \ldots, x_{j}, \ldots\right)$

$$
\begin{aligned}
& =f\left(\ldots, x_{i}+x_{j}, \ldots, x_{i}+x_{j}, \ldots\right)-f\left(\ldots, x_{i}, \ldots, x_{i}, \ldots\right) \\
& -f\left(\ldots, x_{j}, \ldots, x_{j}, \ldots\right)=0
\end{aligned}
$$

$(\Leftarrow)$ Por hipótese, $(i j) \circ f=-f$. Podemos escrever $f=\sum_{\pi \in S_{t}} f_{(t, \pi)}$.

Então $(i j) \circ f_{(t, \pi)}=-f_{(t,(i j) \pi)}, \quad \forall \pi \in S_{t}$.

Consideremos o conjunto $G=\left\{\pi \in S_{t} \mid \pi(i)<\pi(j)\right\}$.

Assim: $f=\sum_{\pi \in G}\left(f_{(t, \pi)}+f_{(t,(i j) \pi)}\right)=\sum_{\pi \in G}\left(f_{(t,(i j) \pi)}-(i j) \circ f_{(t, \pi)}\right)$.

Agora, fazendo $i=j$, seja $f\left(\ldots, x_{i}, \ldots, x_{i}, \ldots\right)$. Temos:

$$
\begin{aligned}
& f\left(\ldots, x_{i}, \ldots, x_{i}, \ldots\right)= \sum_{\pi \in G}\left(f_{(t, \pi)}\left(\ldots, x_{i}, \ldots, x_{i}, \ldots\right)\right. \\
&\left.-(i j) \circ f_{(t, \pi)}\left(\ldots, x_{i}, \ldots, x_{i}, \ldots\right)\right) \\
&=\sum_{\pi \in G}\left(f_{(t, \pi)}\left(\ldots, x_{i}, \ldots, x_{i}, \ldots\right)-f_{(t, \pi)}\left(\ldots, x_{i}, \ldots, x_{i}, \ldots\right)\right)=0
\end{aligned}
$$

Portanto $f$ é normal.

Lema 4.3.2. $f$ é t-normal se e somente se, $\forall \pi \in S_{t}, f_{(t, \pi)}=(\operatorname{sgn} \pi) \pi \circ f_{(t)}$.

Demonstração: Lembrando que $f_{(t, \pi)}$ é a soma dos monômios de $f$ em que as variáveis $x_{1}, \ldots, x_{t}$ aparecem na ordem $\pi_{1}, \ldots, \pi_{t}$. Toda permutação é um produto de transposições. Se $\pi$ for produto de um número par de transposições, $\pi$ é uma permutação par e, será uma permutação ímpar se for produto de um número ímpar de transposições. Seja $\pi \in S_{t}$. Temos que: $\pi=\tau_{1} \ldots \tau_{s}$, onde cada $\tau_{i}$ é uma transposição, $1 \leq i \leq s$.

Pelo lema anterior, $(i j) \circ f=-f$ e, em particular, $(i j) \circ f_{(t)}=-f_{(t)}$, ou especificamente, para a permutação $\pi$ fixada, $\tau_{1} \circ f_{(t)}=-f_{(t)}$. Aplicando sucessivamente $\tau_{2}, \ldots, \tau_{s}$ obtemos: $f_{(t, \pi)}=(\operatorname{sgn} \pi) \pi \circ f_{(t)}$. 
Lema 4.3.3. $f$ é t-normal se e somente se $f=\sum_{\pi \in S_{t}}(\operatorname{sgn} \pi) \pi \circ f_{(t)}$

Demonstração: Resulta imediatamente do fato visto no Lema 4.3.1, de que $f=$ $\sum_{\pi \in S_{t}} f_{(t, \pi)}$ e do lema anterior.

Proposição 4.3.4. Se $f$ é normal de grau $t$ então $f=\alpha s_{t}$ para algum $\alpha \in F$.

Demonstração: Sabemos, da definição do polinômio $f_{(k, \pi)}$, que se $k=t$, então $f=\sum_{\pi \in S_{t}} f_{(t, \pi)}$. Ainda, para cada $\pi \in S_{t}$ :

$f_{(t, \pi)}\left(x_{1}, \ldots, x_{t}\right)=\alpha_{\pi} x_{\pi_{1}} \ldots x_{\pi_{t}}, \quad \alpha_{\pi} \in F$, e se $\pi=1$ :

$f_{(t)}\left(x_{1}, \ldots, x_{t}\right)=\alpha x_{1} \ldots x_{t}, \quad \alpha \in F$.

Pelo Lema 4.3.2, $f_{(t, \pi)}=(\operatorname{sgn} \pi) \pi \circ f_{(t)}$. Então:

$\alpha_{\pi} x_{\pi_{1}} \ldots x_{\pi_{t}}=(\operatorname{sgn} \pi) \pi \circ \alpha x_{1} \ldots x_{t}=(\operatorname{sgn} \pi) \alpha x_{\pi_{1}} \ldots x_{\pi_{t}} \Rightarrow \alpha_{\pi}=(\operatorname{sgn} \pi) \alpha$.

Escrevemos então:

$f=\sum_{\pi \in S_{t}}(\operatorname{sgn} \pi) \alpha x_{\pi_{1}} \ldots x_{\pi_{t}}=\alpha \sum_{\pi \in S_{t}}(\operatorname{sgn} \pi) x_{\pi_{1}} \ldots x_{\pi_{t}} \Rightarrow f=\alpha s_{t}$

Proposição 4.3.5. Seja $f=\sum_{\pi \in S_{2 t}}(\operatorname{sgn} \pi)\left[x_{\pi_{1}}, x_{\pi_{2}}\right] \ldots\left[x_{\pi_{(2 t-1)}}, x_{\pi_{(2 t)}}\right]$. Então

$$
f=2^{t} s_{2 t} \text {. }
$$

Demonstração: Cada comutador se desenvolve numa soma de monômios da forma $x_{\pi_{(i)}} x_{\pi_{(i+1)}}-x_{\pi_{(i+1)}} x_{\pi_{(i)}}$. Fixemos $\pi \in S_{t}$. O produto de todos estes $t$ binômios (pois são $t$ comutadores) é formado por $2^{t}$ parcelas onde em cada uma aparecem todas as $2 t$ variáveis $x_{1}, \ldots, x_{2 t}$ sem repetição. Assim, cada parcela é da forma $x_{\pi_{1}} \ldots x_{\pi_{(2 t)}}$. Para a permutação $\pi$ fixada temos então:

$$
\begin{aligned}
& {\left[x_{\pi_{1}}, x_{\pi_{2}}\right] \ldots\left[x_{\pi_{(2 t-1)}}, x_{\pi_{(2 t)}}\right]=2^{t} x_{\pi_{1}} \ldots x_{\pi_{(2 t)}} } \\
\Rightarrow \quad f & =\sum_{\pi \in S_{2 t}}(\operatorname{sgn} \pi) 2^{t}\left[x_{\pi_{1}}, x_{\pi_{2}}\right] \ldots\left[x_{\pi_{(2 t-1)}}, x_{\pi_{(2 t)}}\right] \\
& =2^{t} \sum_{\pi \in S_{2 t}}(\operatorname{sgn} \pi)\left[x_{\pi_{1}}, x_{\pi_{2}}\right] \ldots\left[x_{\pi_{(2 t-1)}}, x_{\pi_{(2 t)}}\right] \\
\Rightarrow \quad f & =2^{t} s_{2 t}
\end{aligned}
$$

Proposição 4.3.6. Para o polinômio standard $s_{t}$, valem as seguintes igualdades:

(i) $s_{t}=\sum_{i=1}^{t}(-1)^{i-1} x_{i} s_{t-1}\left(x_{1}, \ldots, x_{i-1}, x_{i+1}, \ldots, x_{t}\right)$;

(ii) $s_{t}=\sum_{i=1}^{t}(-1)^{t-1} s_{t-1}\left(x_{1}, \ldots, x_{i-1}, x_{i+1}, \ldots, x_{t}\right) x_{i}$.

\section{Demonstração:}


(i) Faremos indução em $t$. Como, pela própria expressão, é imediato que, sendo ela válida para $k$ é também válida para $k+1$, basta verificar para um valor inicial de $t$, digamos, $t=3$. Teremos:

$$
\begin{aligned}
s_{3}\left(x_{1}, x_{2}, x_{3}\right) & =x_{1} x_{2} x_{3}-x_{1} x_{3} x_{2}+x_{2} x_{1} x_{3}-x_{2} x_{3} x_{1}+x_{3} x_{1} x_{2}-x_{3} x_{2} x_{1} \\
& =x_{1}\left(x_{2} x_{3}\right)-x_{2}\left(x_{1} x_{3}-x_{3} x_{1}\right)+x_{3}\left(x_{1} x_{2}-x_{2} x_{1}\right) \\
& =x_{1} s_{2}\left(x_{1}, x_{2}\right)-x_{2} s_{2}\left(x_{1}, x_{3}\right)+x_{3} s_{2}\left(x_{1} x_{2}\right) \\
& =\sum_{i=1}^{3}(-1)^{i-1} x_{i} s_{2}\left(\ldots, x_{i-1}, x_{i+1}, \ldots\right)
\end{aligned}
$$

(ii) É inteiramente análoga.

Neste ponto introduziremos as matrizes em conjunto com o polinômio standard, e mostraremos duas propriedades do traço de uma matriz. Uma delas será usada na demonstração do Teorema de Amitsur-Levitzki, de vital importância no estudo das identidades.

Lema 4.3.7. Seja $M_{n}(R)$ o anel das matrizes de ordem $n$ sobre um anel comutativo arbitrário $R$. Então $\operatorname{tr}\left[M_{n}(R), M_{n}(R)\right]=0$.

Demonstração: Sejam $A, B \in M_{n}(R)$. Devemos mostrar que $\operatorname{tr}[A, B]=0$. Isto equivale a: $\operatorname{tr}(A B-B A)=0 \Rightarrow \operatorname{tr}(A B)-\operatorname{tr}(B A)=0$, pois o traço é aditivo. Vamos então mostrar que $\operatorname{tr}(A B)=\operatorname{tr}(B A)$.

$$
\begin{aligned}
& A B=(A B)_{i j}=\left(\sum_{k=1}^{n} a_{i k} b_{k j}\right)_{i j} \\
& \operatorname{tr}(A B)=\sum_{k} a_{1 k} b_{k 1}+\cdots+\sum_{k} a_{n k} b_{k n}=\sum_{i=1}^{n} \sum_{k=1}^{n} a_{i k} b_{k i} \\
& B A=(B A)_{i j}=\left(\sum_{k=1}^{n} b_{i k} a_{k j}\right)_{i j} \\
& \operatorname{tr}(B A)=\sum_{i=1}^{n} \sum_{k=1}^{n} b_{i k} a_{k i}=\sum_{i=1}^{n} \sum_{k=1}^{n} a_{k i} b_{i k}=\sum_{k=1}^{n} \sum_{i=1}^{n} a_{k i} b_{i k}=\operatorname{tr}(A B)
\end{aligned}
$$

Proposição 4.3.8. Seja $R$ um anel comutativo arbitrário e $A_{1}, \ldots, A_{2 k} \in M_{n}(R)$. Então $\operatorname{trs}_{2 k}\left(A_{1}, \ldots, A_{2 k}\right)=0$, para $k, n$ arbitrários.

Demonstração: Pelas propriedades do polinômio standard, dadas na Proposição 4.3.6, podemos escrever: 


$$
\begin{aligned}
2 \operatorname{tr} s_{2 k}\left(A_{1}, \ldots, A_{2 k}\right) & =\operatorname{tr}\left(\sum_{i=1}^{2 k}(-1)^{i-1} A_{i} s_{2 k-1}\left(A_{1}, \ldots, A_{i-1}, A_{i}, \ldots, A_{2 k}\right)\right) \\
& +\operatorname{tr}\left(\sum_{i=1}^{2 k}(-1)^{2 k-i} s_{2 k-1}\left(A_{1}, \ldots, A_{i-1}, A_{i}, \ldots, A_{2 k}\right) a_{i}\right)
\end{aligned}
$$

Simplificando um pouco a notação, calculamos:

$$
\begin{aligned}
2 \operatorname{tr} s_{2 k}\left(A_{1}, \ldots, A_{2 k}\right)= & \operatorname{tr}\left(\sum_{i=1}^{2 k}(-1)^{i-1} A_{i} s_{2 k-1}\right)+\operatorname{tr}\left(\sum_{i=1}^{2 k}(-1)^{2 k-i} s_{2 k-1} A_{i}\right) \\
= & \operatorname{tr}\left(A_{1} s_{2 k-1}\right)-\operatorname{tr}\left(s_{2 k-1} A_{1}\right)-\operatorname{tr}\left(A_{2} s_{2 k-1}\right)+\operatorname{tr}\left(s_{2 k-1} A_{2}\right) \\
& +\cdots-\operatorname{tr}\left(A_{2 k} s_{2 k-1}\right)+\operatorname{tr}\left(s_{2 k-1} A_{2 k}\right) \\
= & \operatorname{tr}\left(A_{1} s_{2 k-1}-s_{2 k-1} A_{1}\right)-\operatorname{tr}\left(A_{2} s_{2 k-1}-s_{2 k-1} A_{2}\right) \\
& +\cdots-\operatorname{tr}\left(A_{2 k} s_{2 k-1}-s_{2 k-1} A_{2 k}\right) \\
= & \operatorname{tr}\left[A_{1} s_{2 k-1}, s_{2 k-1} A_{1}\right]-\operatorname{tr}\left[A_{2} s_{2 k-1}-s_{2 k-1} A_{2}\right. \\
& +\cdots-\operatorname{tr}\left[A_{2 k} s_{2 k-1}-s_{2 k-1} A_{2 k}\right]
\end{aligned}
$$

Vemos assim que, quando $i$ é par, $\operatorname{tr}\left[A_{i}, s_{2 k-1}\right]$ é afetado do sinal - , e quando $i$ é ímpar, esta expressão leva o sinal +. Podemos escrever então, voltando à notação de somatório:

$$
2 \operatorname{trs}_{2 k}\left(A_{1}, \ldots, A_{2 k}\right)=\sum_{i=1}^{2 k}(-1)^{i-1} \operatorname{tr}\left[A_{i}, s_{2 k-1}\left(A_{1}, \ldots, A_{i-1}, A_{i}, \ldots, A_{2 k}\right)\right]
$$

De acordo com o Lema 4.3.7 o segundo membro se anula, pois é uma soma do traço do comutador de matrizes em $M_{n}(R)$. Portanto $\operatorname{tr} s_{2 k}\left(A_{1}, \ldots, A_{2 k}\right)=0$.

Os resultados que acabamos de apresentar formam o embasamento teórico para o estudo das identidades nos octônios. Para completar tais fundamentos necessitamos do Teorema de Amitsur-Levitzki, que afirma que o polinômio standard $s_{2 n}$ é uma identidade em $M_{n}(R)$ (ou $M_{n}(F), F$ corpo). Daremos a demonstração devida a Rosset (ref. [20]), que considera este teorema como uma conseqüência do Teorema de Cayley-Hamilton para matrizes. Esta demonstração faz uso de resultados referentes a álgebra exterior. Para isto dedicaremos a próxima seção a um estudo sucinto desta álgebra. Maiores detalhes podem ser encontrados em [23].

\subsection{A Álgebra Exterior - Principais Resultados}

Sejam $U, V$ espaços vetoriais sobre um mesmo corpo $F, \operatorname{com} \operatorname{dim} U=m$ e $\operatorname{dim} V=n$. Queremos definir um "espaço produto" entre $U$ e $V$. A primeira idéia que poderia nos 
ocorrer seria o produto cartesiano $U \times V$. Mas, notando que, se $\left(u_{1}, v\right),\left(u_{2}, v\right) \in U \times V$, então $\left(u_{1}, v\right)+\left(u_{2}, v\right) \neq\left(u_{1}+u_{2}, v\right)$. Mas se $w$ for uma função bilinear, $w: U \times V \rightarrow F$, então, para todos $u, u_{1}, u_{2} \in U, v, v_{1}, v_{2} \in V$ e $a \in F$ :

(i) $w\left(u_{1}+u_{2}, v\right)=w\left(u_{1}, v\right)+w\left(u_{2}, v\right)$;

(ii) $w\left(u, v_{1}+v_{2}\right)=w\left(u, v_{1}\right)+w\left(u, v_{2}\right)$;

(iii) $w(a u, v)=w(u, a v)=a w(u, v)$.

Define-se o produto tensorial de $U$ por $V$ como sendo o espaço dual do espaço das funções bilineares de $U \times V$ em $F$. Denotamos por $U \otimes V$ :

$$
U \otimes V=L^{*}(U \times V ; F)=L(L(U \times V ; F) ; F)
$$

Assim, os elementos de $U \otimes V$ são funcionais lineares em $L(U \otimes V ; F)$, e tem-se $\operatorname{dim} U \otimes V=\operatorname{dim} L(U \otimes V ; F)=m n$. Dos resultados conhecidos sobre funcionais lineares, sabemos que, se $\left\{u_{1}, \ldots, u_{m}\right\}$ é uma base de $U$ e $\left\{v_{1}, \ldots, v_{n}\right\}$ é uma base de $V$, então $\left\{\alpha_{i j}\right\}, i=1, \ldots, m, j=1, \ldots n$, é uma base de $L(U \otimes V ; F)$, onde $\alpha_{i j}$ é um funcional bilinear definido por:

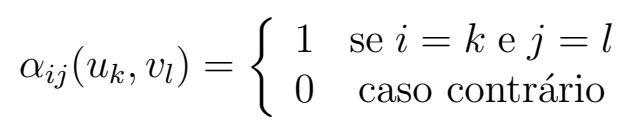

Definimos $\left(u_{i} \otimes v_{j}\right)\left(\alpha_{k l}\right)=\alpha_{k l}\left(u_{i}, v_{j}\right)$. Assim, $\left(u_{i} \otimes v_{j}\right), 1 \leq i \leq m, 1 \leq j \leq n$, é uma base de $U \otimes V$. Por exemplo, se $m=2$ e $n=3$, então $U \otimes V$ tem dimensão 6 e sua base será:

$$
\left\{u_{1} \otimes v_{1}, u_{1} \otimes v_{2}, u_{1} \otimes v_{3}, u_{2} \otimes v_{1}, u_{2} \otimes v_{2}, u_{2} \otimes v_{3}\right\}
$$

Um elemento de $U \otimes V$ é escrito como $\sum_{i j} a_{i j}\left(u_{i} \otimes v_{j}\right)$.

Estendemos naturalmente a definição de produto tensorial para um número maior de espaços vetoriais. Sejam, então $U_{1}, \ldots, U_{r}$ espaços vetoriais de dimensão finita. Uma função multilinear em $r$ variáveis é uma aplicação $\alpha: U_{1} \times \ldots \times U_{r} \rightarrow F$, que é linear em cada variável, sendo também chamada de uma $r$-forma. Assim, podemos considerar que um funcional linear é uma 1-forma, um funcional bilinear é uma 2forma, e assim por diante. Também chamamos de uma 0-forma, um elemento do corpo $F$. 
O espaço vetorial de todas as r-formas é $L\left(U_{1}, \ldots, U_{r} ; F\right)$ e o seu dual é o espaço tensorial $U_{1} \otimes \ldots \otimes U_{r}$.

Consideremos agora as r-formas do tipo

$$
\alpha: V \times \ldots \times V \rightarrow F
$$

O espaço vetorial de todas as formas deste tipo é $L\left(V^{r} ; F\right)$ e o seu dual é chamado espaço tensorial contravariante de ordem $r$, denotado por $\stackrel{r}{\otimes} V$. Se $\operatorname{dim} V=n$, então $\operatorname{dim} \stackrel{r}{\otimes} V=n^{r}$.

Sejam $v_{1}, \ldots, v_{r} \in V$. Uma $\mathrm{r}$-forma $\alpha$ pode ser calculada nestes $v_{i}$, resultando $\alpha\left(v_{1}, \ldots, v_{r}\right)=k, k \in F$. Poderíamos perguntar o que acontece quando permutamos os $v_{i}$, isto é, quando calculamos $\alpha\left(v_{\pi_{1}}, \ldots, v_{\pi_{r}}\right)$, sendo $\pi \in S_{r}$, o grupo das permutações de $r$ elementos. Interessam-nos somente as r-formas que satisfazem as seguintes condições:

1. Uma r-forma $\alpha$ em $V$ é dita simétrica se

$$
\alpha\left(v_{1}, \ldots, v_{r}\right)=\alpha\left(v_{\pi_{1}}, \ldots, v_{\pi_{r}}\right), \quad \forall v_{i} \in V \forall \pi \in S_{r}
$$

2. Uma r-forma $\alpha$ em $V$ é dita anti-simétrica se

$$
\begin{aligned}
& \alpha\left(v_{1}, \ldots, v_{r}\right)=\operatorname{sgn}(\pi) \alpha\left(v_{\pi_{1}}, \ldots, v_{\pi_{r}}\right), \forall v_{i} \in V \forall \pi \in S_{r}, \\
& \text { onde } \operatorname{sgn}(\pi) \text { é o sinal de } \pi .
\end{aligned}
$$

3. Uma r-forma $\alpha$ em $V$ é dita alternada se for igual a 0 sempre que houver duas variáveis repetidas.

Toda forma alternada é anti-simétrica. Se o corpo $F$ tem característica $\neq 2$, então toda forma anti-simétrica é também alternada. De fato, seja $\alpha$ anti-simétrica. Então $\alpha\left(v_{1}, v_{1}, \ldots, v_{r}\right)=-\alpha\left(v_{1}, v_{1}, \ldots, v_{r}\right) \Rightarrow 2 \alpha\left(v_{1}, v_{1}, \ldots, v_{r}\right)=0$. E como $F$ não é de característica 2 , segue que $\alpha\left(v_{1}, v_{1}, \ldots, v_{r}\right)=0$. Portanto $\alpha$ é alternada.

O conjunto das $r$-formas simétricas em $V$, denotado por $L_{S}\left(V^{r} ; F\right)$ é um subespaço de $L\left(V^{r} ; F\right)$, como se verifica facilmente. Da mesma maneira o conjunto das r-formas alternadas em $V$, denotado por $L_{A}\left(V^{r} ; F\right)$ é um subespaço de $L\left(V^{r} ; F\right)$. Seja $\left\{v_{i}\right\}$ uma base de $V$. É possível provar que a dimensão de $L_{A}\left(V^{r} ; F\right)$ é dada por 


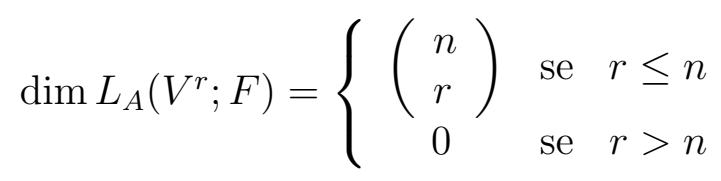

Definição 4.4.1. Seja $V$ um espaço vetorial de dimensão n. O produto exterior de ordem $\boldsymbol{r}$ de $V$ é o dual das r-formas alternadas, $L_{A}\left(V^{r} ; F\right)$, denotado por $\stackrel{r}{\wedge} V$. Em símbolos: $\wedge^{r} V=L_{A}^{*}\left(V^{r} ; F\right)=L\left(L_{A}\left(V^{r} ; F\right) ; F\right)$.

Observação: A definição acima é restrita para característica zero. Para uma definição mais geral que independe da característica do corpo, veja, por exemplo, Jacobson (ref. $[13])$.

Tem-se também: $\operatorname{dim} L_{A}\left(V^{r} ; F\right)=\left(\begin{array}{c}n \\ r\end{array}\right) \Rightarrow \stackrel{r}{\wedge} V=\left(\begin{array}{c}n \\ r\end{array}\right)$

Sejam $x_{1}, \ldots, x_{r}$ elementos fixados de $V$, e $\alpha$ uma r-forma em $L_{A}\left(V^{r} ; F\right)$. Um elemento de $\stackrel{r}{\wedge} V$ é um funcional linear (1-forma) que associa a cada $\alpha \in L_{A}\left(V^{r} ; F\right)$, um escalar. Assim, se definirmos

$$
\begin{aligned}
\beta: L_{A}\left(V^{r} ; F\right) & \rightarrow F \\
\alpha & \mapsto \beta(\alpha)=\alpha\left(x_{1}, \ldots, x_{r}\right)
\end{aligned}
$$

então $\beta \in \stackrel{r}{\wedge} V$. Um elemento $\beta$ de $\stackrel{r}{\wedge} V$ é uma 1-forma, como definido em (1), e é chamado produto exterior de $x_{1}, \ldots, x_{r}$. Usamos a notação $\beta=x_{1} \wedge \ldots \wedge x_{r}$. Assim, podemos reescrever (1):

$$
\left(x_{1} \wedge \ldots \wedge x_{r}\right)(\alpha)=\alpha\left(x_{1}, \ldots, x_{r}\right)
$$

É possível provar que se $\left\{v_{1}, \ldots, v_{r}\right\}$ é uma base de $V$, então o conjunto de $\left(\begin{array}{c}n \\ r\end{array}\right)$ vetores $v_{i_{1}} \wedge v_{i_{2}} \wedge \ldots \wedge v_{i_{r}}$, com $1 \leq i_{1}<i_{2}<\ldots<i_{r} \leq n$ é uma base de $\stackrel{r}{\wedge} V$. Vejamos alguns exemplos:

1. Se $r=2$ e $n=3,\left(\begin{array}{l}3 \\ 2\end{array}\right)=3 ;\left\{v_{1}, v_{2}, v_{3}\right\}$ é base de $V$ e $\left\{v_{1} \wedge v_{2}, v_{1} \wedge v_{3}, v_{2} \wedge v_{3}\right\}$ é base de $\stackrel{2}{\wedge} V$. Todo elemento de $\stackrel{2}{\wedge} V$ é da forma

$$
x=\lambda_{12} v_{1} \wedge v_{2}+\lambda_{13} v_{1} \wedge v_{3}+\lambda_{23} v_{2} \wedge v_{3}, \quad \lambda_{i j} \in F .
$$

2. Se $r=2$ e $n=4,\left(\begin{array}{l}4 \\ 2\end{array}\right)=6$, daí: $\left\{v_{1} \wedge v_{2}, v_{1} \wedge v_{3}, v_{1} \wedge v_{4}, v_{2} \wedge v_{3}, v_{2} \wedge v_{4}, v_{3} \wedge v_{4}\right\}$ é base de $\stackrel{2}{\wedge} V$. Um elemento $x \in V \times V$ tem a forma 


$$
\sum_{i, j} \lambda_{i j}\left(v_{i} \wedge v_{j}\right), \quad 1 \leq i<j \leq 4, \lambda_{i j} \in F
$$

3. Se $r=3$ e $n=4,\left(\begin{array}{l}4 \\ 3\end{array}\right)=4$, e uma base de $\stackrel{3}{\wedge} V$ é $\left\{v_{1} \wedge v_{2} \wedge v_{3}, v_{1} \wedge v_{2} \wedge v_{4}, v_{1} \wedge v_{3} \wedge v_{4}, v_{2} \wedge v_{3} \wedge v_{4}\right\}$

Um elemento de $\stackrel{r}{\wedge} V$ é chamado um r-vetor. Se um elemento de $\stackrel{r}{\wedge} V$ puder ser escrito como produto exterior de $r$ elementos de $V$, então tal elemento é chamado um r-vetor simples. Usa-se a notação $v^{(r)}$ para indicar um r-vetor simples.

Consideremos agora dois produtos exteriores, $\stackrel{r}{\wedge} V$ e $\stackrel{s}{\wedge} V$, com $r \neq s$. Sejam $v^{(r)} \in \stackrel{r}{\wedge} V$ um r-vetor simples e $v^{(s)} \in \stackrel{s}{\wedge} V$ um s-vetor simples. Podemos escrever:

$$
v^{(r)}=x_{1} \wedge \ldots \wedge x_{r} \quad \text { e } \quad v^{(s)}=x_{r+1} \wedge \ldots \wedge x_{r+s}
$$

com $x_{i} \in V, i=1, \ldots, r+s$. Vamos definir o produto exterior de $v^{(r)}$ por $v^{(s)}$ como

$$
v^{(r)} \wedge v^{(s)}=x_{1} \wedge \ldots \wedge x_{r} \wedge x_{r+1} \wedge \ldots \wedge x_{r+s}
$$

Claro que esta definição só tem sentido se $r+s \leq n$, onde $n=\operatorname{dim} V$. Mas podemos ter elementos em $\stackrel{r}{\wedge} V$ (e em $\stackrel{s}{\wedge} V$ ) que não são simples. Porém são combinações lineares de elementos da base de $\stackrel{r}{\wedge} V$, isto é, de r-vetores simples. Assim, qualquer vetor $v^{(r)} \in \stackrel{r}{\wedge} V$ pode ser escrito:

$$
v^{(r)}=\sum_{i=1}^{\left(\begin{array}{c}
n \\
r
\end{array}\right)} \lambda_{i} v_{i}^{(r)}, \quad \lambda_{i} \in F .
$$

onde cada elemento da base de $\stackrel{r}{\wedge} V$ foi designado por $v_{i}^{(r)}$. Como um exemplo, se $r=3$ e $n=4$, a base de $\stackrel{3}{\wedge} V$ é

$$
\left\{v_{1}^{(3)}=v_{1} \wedge v_{2} \wedge v_{3}, v_{2}^{(3)}=v_{1} \wedge v_{2} \wedge v_{4}, v_{3}^{(3)}=v_{1} \wedge v_{3} \wedge v_{4}, v_{4}^{(3)}=v_{2} \wedge v_{3} \wedge v_{4}\right\}
$$

Um elemento qualquer de $\stackrel{3}{\wedge} V$ é um 3 -vetor, que pode ser escrito como:

$$
v^{(3)}=\sum_{i=1}^{4} \lambda_{i} v_{i}^{(3)}, \quad \lambda_{i} \in F
$$

Vamos definir o produto exterior de um r-vetor por um s-vetor, estendendo a definição dada para vetores simples. Sejam $v^{(r)}=\sum_{i} \lambda_{i} v_{i}^{(r)}$ e $v^{(s)}=\sum_{j} \mu_{j} v_{j}^{(s)}, \quad \lambda_{i}, \mu_{j} \in$ F. Definimos:

$$
v^{(r)} \wedge v^{(s)}=\left(\sum_{i} \lambda_{i} v_{i}^{(r)}\right) \wedge\left(\sum_{j} \mu_{j} v_{j}^{(s)}\right)=\sum_{i, j} \lambda_{i} \mu_{j}\left(v_{i}^{(r)} \wedge v_{j}^{(s)}\right)
$$


É possível provar que:

1. Esta definição é válida, isto é, independe de como $v^{(r)}$ e $v^{(s)}$ são escritos em termos de vetores simples;

2. $v^{(r)} \wedge v^{(s)}=(-1)^{r s} v^{(s)} \wedge v^{(r)}$.

Seja $V$ um F-espaço vetorial de dimensão $n$. Definimos:

$$
\wedge V={ }^{\wedge} V \oplus \stackrel{1}{\wedge} V \oplus \cdots \oplus \wedge^{n} V=\bigoplus_{r=0}^{n} \wedge^{r} V
$$

onde $\stackrel{0}{\wedge} V=F$ e $\stackrel{1}{\wedge} V=V$. Tem-se ainda:

$$
\operatorname{dim} \wedge V=\left(\begin{array}{c}
n \\
0
\end{array}\right)+\left(\begin{array}{c}
n \\
1
\end{array}\right)+\cdots+\left(\begin{array}{c}
n \\
n
\end{array}\right)=2^{n}
$$

Vamos definir um produto em $\wedge V$. Sejam $v_{1}=\bigoplus_{r=0}^{n} v_{1}^{(r)}$ e $v_{2}=\bigoplus_{s=0}^{n} v_{2}^{(r)}$. Definimos: $v_{1} \wedge v_{2}=\bigoplus_{t=o}^{n} \sum_{r+s=t} v_{1}^{(r)} \wedge v_{2}^{(s)}$

É fácil verificar que $\wedge V$ com o produto assim definido é uma álgebra. A álgebra $\wedge V$ assim constituída é chamada álgebra exterior ou álgebra de Grassmann. Vejamos alguns exemplos:

1. $n=3:\left\{e_{1}, e_{2}, e_{3}\right\}$ base de $V, \wedge V=F \oplus V \oplus \stackrel{2}{\wedge} V \oplus \stackrel{3}{\wedge} V, \quad \operatorname{dim} \wedge V=2^{3}=8$.

Sejam $v_{1}, v_{2} \in \wedge V$. Vamos explicitar cada um deles como soma direta de elementos de cada $\wedge^{i} V, i=1,2,3,4$. Assim, na notação indicada anteriormente, $v_{1}=v_{1}^{(0)} \oplus v_{1}^{(1)} \oplus v_{1}^{(2)} \oplus v_{1}^{(3)}$ e $v_{2}=v_{2}^{(0)} \oplus v_{2}^{(1)} \oplus v_{2}^{(2)} \oplus v_{2}^{(3)}$, onde

(i) $\quad v_{1}^{(0)} \in F \quad \Rightarrow \quad v_{1}^{(0)}=\lambda$

(ii) $v_{1}^{(1)} \in V \quad \Rightarrow \quad v_{1}^{(1)}=\lambda_{11} e_{1}+\lambda_{12} e_{2}+\lambda_{13} e_{3}$

(iii) $v_{1}^{(2)} \in \stackrel{2}{\wedge}^{\wedge} \Rightarrow v_{1}^{(2)}=\lambda_{21} e_{1} \wedge e_{2}+\lambda_{22} e_{1} \wedge e_{3}+\lambda_{23} e_{2} \wedge e_{3}$

(iv) $v_{1}^{(3)} \in \stackrel{3}{\wedge} V \Rightarrow v_{1}^{(3)}=\lambda_{31} e_{1} \wedge e_{2} \wedge e_{3}$

onde $\lambda, \lambda_{i j} \in F, \quad i, j=1,2,3$.

Naturalmente temos expressões análogas para $v_{2}$. Assim, uma base de $\wedge V$, para $\operatorname{dim} V=3$ é:

$$
\left\{1, \quad e_{1}, \quad e_{2}, \quad e_{3}, \quad e_{1} \wedge e_{2}, \quad e_{1} \wedge e_{3}, \quad e_{2} \wedge e_{3}, \quad e_{1} \wedge e_{2} \wedge e_{3}\right\}
$$


Vamos calcular o produto $v_{1} \wedge v_{2}$ de acordo com a definição dada:

$$
\begin{aligned}
v_{1} \wedge v_{2}= & \bigoplus_{t=o}^{3} \sum_{r+s=t} v_{1}^{(r)} \wedge v_{2}^{(s)} \\
= & v_{1}^{(0)} \wedge v_{2}^{(0)} \oplus v_{1}^{(0)} \wedge v_{2}^{(1)} \oplus v_{1}^{(1)} \wedge v_{2}^{(0)} \oplus v_{1}^{(0)} \wedge v_{2}^{(2)} \oplus v_{1}^{(2)} \wedge v_{2}^{(0)} \oplus v_{1}^{(1)} \wedge v_{2}^{(1)} \\
& \oplus v_{1}^{(0)} \wedge v_{2}^{(3)} \oplus v_{1}^{(1)} \wedge v_{2}^{(2)} \oplus v_{1}^{(2)} \wedge v_{2}^{(1)} \oplus v_{1}^{(3)} \wedge v_{2}^{(0)}
\end{aligned}
$$

2. Se $n=4$, o produto $v_{1} \wedge v_{2}$ apresenta os mesmos termos acima mais os termos correspondentes a $t=4$. Indicamos sinteticamente:

$$
\begin{aligned}
v_{1} \wedge v_{2}= & \stackrel{(t=0)}{\cdots} \oplus \stackrel{(t=1)}{\cdots} \oplus \stackrel{(t=2)}{\cdots} \oplus \stackrel{(t=3)}{\cdots} \\
& \oplus v_{1}^{(0)} \wedge v_{2}^{(4)} \oplus v_{1}^{(1)} \wedge v_{2}^{(3)} \oplus v_{1}^{(2)} \wedge v_{2}^{(2)} \oplus v_{1}^{(3)} \wedge v_{2}^{(1)} \oplus v_{1}^{(4)} \wedge v_{2}^{(0)}
\end{aligned}
$$

Encerramos esta seção com a demonstração do Teorema de Amitsur-Levitzki. Precisaremos ainda de um lema que trata das fórmulas de Newton para matrizes genéricas. Daremos apenas uma idéia da sua demonstração, cujos detalhes podem ser vistos em [21].

Lema 4.4.1 (Fórmulas de Newton). Seja $A \in M_{n}(F) e(-1)^{k} \alpha_{k}$ o coeficiente de $\lambda^{n-k}$ no polinômio característico da matriz $A$, então:

$$
k \alpha_{k}=\sum_{i=1}^{k}(-1)^{i-1} \alpha_{k-i} \operatorname{tr}\left(A^{i}\right), \quad \forall k, 1 \leq k \leq n .
$$

Demonstração: (idéia) Considera o polinômio característico $q$ da matriz $A$ na variável $\lambda$. Calcula a derivada formal $q^{\prime}$ em relação a $\lambda$ e a desenvolve em série de potências. Deste desenvolvimento obtém-se as fórmulas de Newton.

Teorema 4.4.2 (Amitsur-Levitzki). O polinômio standard $s_{2 n}$ é uma identidade na álgebra $M_{n}(F)$ das matrizes de ordem $n$ sobre um corpo $F$.

Demonstração: Seja $V$ espaço vetorial de dimensão $2 n$ sobre um corpo $F$ com car $F=0$. Para facilitar a notação vamos indicar por $E$ a álgebra exterior de $V$, ao invés de $\wedge V$. Seja $\left\{e_{1}, \ldots, e_{2 n}\right\}$ uma base de $V$ e consideremos $2 n$ matrizes $A_{1}, \ldots, A_{2 n}$ em $M_{n}(F)$. É possível associar o produto tensorial $M_{n}(F) \otimes E$ com $M_{n}(E)$, obtendo matrizes de ordem $n$ cujas entradas são indeterminadas, mais especificamente, elementos da forma $\lambda e_{i}, \operatorname{com} \lambda \in F, e_{i} \in E$. Esta associação é feita calculando: $A=A_{1} e_{1}+\cdots+A_{2 n} e_{2 n} \in M_{n}(E)$. Calculemos as potências da matriz genérica $A$, para o caso particular $n=2$. Temos: 


$$
\begin{aligned}
A^{2}= & \left(A_{1} A_{2}-A_{2} A_{1}\right) e_{1} \wedge e_{2}+\left(A_{1} A_{3}-A_{3} A_{1}\right) e_{1} \wedge e_{3}+\left(A_{1} A_{4}-A_{4} A_{1}\right) e_{1} \wedge e_{4} \\
& +\left(A_{2} A_{3}-A_{3} A_{2}\right) e_{2} \wedge e_{3}+\left(A_{2} A_{4}-A_{4} A_{1}\right) e_{2} \wedge e_{4}+\left(A_{3} A_{4}-A_{4} A_{3}\right) e_{3} \wedge e_{4} \\
A^{3}= & \left(A_{1} A_{2} A_{3}-A_{2} A_{1} A_{3}\right) e_{1} \wedge e_{2} \wedge e_{3}+\left(A_{1} A_{2} A_{4}-A_{2} A_{1} A_{4}\right) e_{1} \wedge e_{2} \wedge e_{4}+\cdots \\
A^{4}= & \left(A_{1} A_{2} A_{3} A_{4}-A_{2} A_{1} A_{3} A_{4}+A_{1} A_{3} A_{4} A_{2}-A_{2} A_{4} A_{3} A_{1}+\cdots\right) e_{1} \wedge e_{2} \wedge e_{3} \wedge e_{4}
\end{aligned}
$$

É possível concluir então que, para um $n$ qualquer, teremos:

$A^{2 n}=s_{2 n}\left(A_{1}, \ldots, A_{2 n}\right) e_{1} \wedge e_{2} \wedge \cdots e_{2 n}$

Para mostrar que $s_{2 n}$ é uma identidade em $M_{n}(F)$, basta mostrar que $A^{2 n}=$ 0. Consideremos então as fórmulas de Newton para matrizes genéricas, que dão os coeficientes do polinômio característico de uma matriz $A$ em função do traço das potências de $A$. Como vimos no lema anterior, tais fórmulas podem ser escritas:

$$
k \alpha_{k}=\sum_{i=1}^{k}(-1)^{i-1} \alpha_{k-i} \operatorname{tr}\left(A^{i}\right), \quad \forall k, 1 \leq k \leq n
$$

Com isto, se mostrarmos que $\operatorname{tr}\left(A^{i}\right)=0$ para todo $i$, teremos, pelo Teorema de Cayley-Hamilton, que $A^{2 n}=0$. No caso em estudo as potências da matriz $A$ têm a forma

$$
A^{k}=\sum_{i_{1}<\ldots<i_{k}} s_{k}\left(A_{i_{1}}, \ldots, A_{i_{k}}\right) e_{i_{1}} \wedge \ldots \wedge e_{i_{k}}
$$

como podemos facilmente comprovar das expressões indicadas acima. Mas queremos $A^{2 n}=0$. Sabemos pela Proposição 4.3 .8 que o traço da matriz $s_{2 n}\left(A_{1}, \ldots, A_{2 n}\right)$ é igual a zero, para todo $n$. Daí segue o resultado.

Corolário 4.4.3. Todo polinômio $f$ normal de grau 4 é uma identidade em $M_{2}(F)$.

Demonstração: Pela Proposição 4.3.4, $f=\alpha s_{4}$. Mas, pelo Teorema, $s_{4}$ é uma identidade em $M_{2}(F)$, logo $f$ é uma identidade em $M_{2}(F)$.

O próximo resultado estabelece uma espécie de recíproca do Teorema de AmitsurLevitzki. Sua demonstração pode ser encontrada em [21].

Teorema 4.4.4. (i) A álgebra das matrizes $M_{n}(F)$ não satisfaz qualquer identidade polinomial de grau menor que $2 n$.

(ii) Se $f$ é uma identidade multilinear de $M_{n}(F)$ de grau $2 n$ então, para algum $\alpha \in F, f=\alpha S_{2 n}$. 


\subsection{Identidades nos Octônios - Grau 4}

Mostraremos nesta seção que não existem identidades de grau 4 ou menor na álgebra dos octônios, que não sejam conseqüências das leis alternativas à direita e à esquerda. Identidades que são conseqüências das duas leis alternativas são chamadas identidades triviais (ou óbvias).

Conforme notação introduzida no final do capítulo anterior, designaremos por $\mathbb{O}$ a álgebra dos octônios. Dada uma identidade $f$ em $\mathbb{O}$, podemos agrupar os monômios do mesmo tipo. Cada grupo destes, ou seja, cada soma de monômios de um certo tipo produz um polinômio homogêneo deste tipo. A identidade $f$ será então, uma soma de polinômios homogêneos, digamos $f=f_{1}+f_{2}+\cdots+f_{t}$. Os polinômios $f_{i}$ são chamados componentes homogêneos de $f$. Com isto, algumas importantes simplificações podem ser feitas, conforme indicamos a seguir.

Teorema 4.5.1. Seja A uma F-álgebra. Se o corpo F tem mais elementos que o grau máximo de qualquer variável de uma identidade $f$ em A, então todo componente homogêneo de f é uma identidade.

Demonstração: (idéia) Seja $t$ o grau de $f$. A essência do teorema está em provar que: Se existirem escalares $\alpha_{1}, \ldots, \alpha_{t}$ tais que, para o determinante de Vandermonde $d$ formado pelas potências de $\alpha_{i}$, a equação $d a=0$ implica $a=0$, para todo $a \in A$, então todo componente homogêneo da identidade $f$ é também uma identidade em $A$. Escreve $f$ como uma soma de polinômios $f=f_{0}+f_{1}+\cdots+f_{j}$, onde em cada $f_{i}$, a variável $x_{1}$ tem grau $i$. Supondo $f$ um polinômio em $n$ variáveis, tomamse $a_{1}, \ldots, a_{n} \in A$, elementos arbitrários. Para cada $i=1, \ldots, j+1$, a expressão $f\left(\alpha_{i} a_{1}, a_{2}, \ldots, a_{n}\right)=0$, implica que $d_{1} f_{k}(a)=0$, onde $a=\left(a_{1}, \ldots, a_{n}\right), k=0,1, \ldots, j$ e $d_{1}$ é o determinante de Vandermonde formado por $\alpha_{1}, \ldots, \alpha_{j+1}$. Finalmente, sendo $d_{1}$ divisor de $d$, a equação $d_{1} f_{k}(a)=0$ implica que $f_{k}(a)=0$ e, portanto, que $f_{0}, f_{1}, \ldots, f_{j}$ são identidades em $A$.

Note que, como estamos num corpo $F$ cuja característica é maior que o grau da identidade, as condições do teorema são verificadas. Para maiores detalhes, veja a referência [25].

Vamos verificar que podemos assumir que uma identidade homogênea é também alternada nas variáveis lineares. Ordene os componentes homogêneos de uma iden- 
tidade $f$, primeiro pelo grau e depois pelo número de variáveis. Então, se $y$ e $z$ são variáveis lineares e se $f_{k}(\ldots, y, z, \ldots)+f_{k}(\ldots, z, y, \ldots)$ não é o polinômio nulo, então, fazendo $z=y$, obtemos $f_{k}(\ldots, y, y, \ldots)$ também não nulo e com menor número de variáveis. Supondo que conhecemos os resultados sobre identidades alternadas mais baixas na nossa classificação (isto é, com menor número de variáveis), vamos linearizar $f_{k}(\ldots, y, y, \ldots)$, obtendo

$$
f_{k}(\ldots, y, z, \ldots)+f_{k}(\ldots, z, y, \ldots)
$$

Mas

$$
f_{k}(\ldots, y, z, \ldots)-f_{k}(\ldots, z, y, \ldots)
$$

é uma identidade alternada em $y$ e $z$. Somando as identidades (1) e (2) obtemos:

$$
2 f_{k}(\ldots, y, z, \ldots)
$$

Portanto, podemos assumir que $f$ é alternada em $y$ e $z$.

Uma outra simplificação que podemos obter se refere ao isomorfismo entre os quatérnios e as matrizes $2 \times 2$, conforme mostramos no próximo lema.

Lema 4.5.2. Os quatérnios $Q(0,1)$ e as matrizes $M_{2}[F]$ são isomorfos.

Demonstração: Seja $a \in Q$. Então $a=a_{0} .1+a_{1} e_{1}+a_{2} e_{2}+a_{3} e_{3}$, onde $a_{i} \in F, i=$ $0, \ldots, 3$. Podemos escrever também, $a=\left(a_{0}, a_{1}, a_{2}, a_{3}\right)$. Considere a aplicação

$$
\begin{aligned}
\varphi: Q & \rightarrow M_{2}(F) \\
\varphi\left(a_{0}, a_{1}, a_{2}, a_{3}\right) & \mapsto\left(\begin{array}{cc}
a_{0}+a_{1} & a_{2}+a_{3} \\
a_{2} & a_{0}
\end{array}\right)
\end{aligned}
$$

$\varphi$ é claramente um isomorfismo.

Precisaremos ainda de algumas importantes propriedades do associador em uma álgebra alternativa, que são dadas no seguinte lema operacional.

Lema 4.5.3. Em uma álgebra alternativa $A$ valem as seguintes igualdades, para todos $x, y, z \in A$ :

(i) $(x, x y, z)=(x, y, z) x$;

(ii) $(x, y x, z)=x(x, y, z)$; 
(iii) $\left(x, y, z^{2}\right)=z \circ(x, y, z)$.

\section{Demonstração:}

(i) Note que: $(x, x y, z)=-(z, x y, x)$ e que $(x, y, z)=(z, x, y)$. Então:

$$
\begin{aligned}
(z, x y, x) & =(z(x y)) x-z(x y x) \stackrel{*}{=}(z(x y)) x-((z x) y) x \\
& =(z(x y)-(z x) y) x=-(z, x, y) x \Rightarrow(x, x y, z)=(x, y, z) x
\end{aligned}
$$

(*) Moufang (iii) (Teorema 1.2.2)

(ii) $(x, y x, z)=(x y x) z-x((y x) z) \stackrel{* *}{=} x(y(x z))-x((y x) z)=x(-(y, x, z))$

$$
=x(x, y, z)
$$

(**) Moufang (i)

(iii) Em uma álgebra alternativa, temos a seguinte identidade: $\left(x^{2}, y, x\right)=0$, que resulta da lei flexível, como mostramos a seguir:

$\left(x^{2}, y, x\right)=\left(x^{2} y\right) x-x^{2}(y x)=(x(x y)) x-x(x(y x))=x((x y) x)-x((x y) x)=0$.

Linearizando esta expressão com $x \rightarrow x+\lambda z$ :

$$
\begin{aligned}
\begin{aligned}
&\left((x+\lambda z)^{2}, y, x+\lambda z\right)=\left(x^{2}+\lambda x z+\lambda z x+\lambda^{2} z^{2}, y, x+\lambda z\right) \\
&=\underbrace{\left(x^{2}, y, x\right)}_{=0}+\lambda\left(x^{2}, y, z\right)+\lambda(x z, y, x)+\lambda^{2}(x z, y, z) \\
&+\lambda(z x, y, x)+\lambda^{2}(z x, y, z)+\lambda^{2}\left(z^{2}, y, x\right)+\underbrace{\lambda^{3}\left(z^{2}, y, z\right)}_{=0} \\
&=\lambda\left[\left(x^{2}, y, z\right)+(x z, y, x)+(z x, y, x)\right]+\lambda^{2}\left[\left(z^{2}, y, x\right)+(z x, y, z)+(x z, y, z)\right]=0
\end{aligned}
\end{aligned}
$$

Note que os coeficientes de $\lambda$ e de $\lambda^{2}$ são equivalentes, bastando trocar $x$ por $z$ e $z$ por $x$. Portanto, tomando a expressão para $\lambda^{2}$ :

$\left(z^{2}, y, x\right)+(z x, y, z)+(x z, y, z)=0 \Rightarrow\left(x, y, z^{2}\right)=(x \circ z, y, z)$

Fazendo $y=z$ e $z=y$ em (i) e (ii) e somando:

$(x, x z, y)+(x, z x, y)=(x, y, z) \circ x, \quad$ que também pode ser escrito:

$(x, x \circ z, y)=x \circ(x, z, y)$. Agora, trocando $x$ por $z$ e $z$ por $x$, obtemos:

$(z, x \circ z, y)=z \circ(z, x, y), \quad$ o que equivale a:

$(x \circ z, y, z)=z \circ(x, y, z)$

Comparando (I) e (II) obtemos: $\left(x, y, z^{2}\right)=z \circ(x, y, z)$ 
Um polinômio homogêneo do grau 4 pode ser de um dos seguintes tipos: [4], $[3,1],[2,2],[2,1,1]$ e $[1,1,1,1]$. Por exemplo, $x^{4}$ é um polinômio do tipo [4]. Do tipo $[3,1]$, temos $x^{3} y$ e $x^{2}(y x)$. Já $x^{2} y^{2}$ e $\left(x y^{2}\right) x$ são do tipo $[2,2]$. Do tipo $[2,1,1]$, são identidades em 3 variáveis $x, y$ e $z$, sendo do grau 2 em $x$ e do grau 1 em $y$ e $z$. Como exemplo citamos: $x(x y z)$ e $(x y)(z x)$. Finalmente o tipo $[1,1,1,1]$ se refere às identidades multilineares em 4 variáveis, como $x((y z) w)$ ou $(x y)(z w)$.

Os três primeiros tipos são associativos, pois têm apenas 1 ou 2 variáveis, logo pelo Teorema de Artin, geram uma subálgebra associativa. Uma identidade deste tipo é portanto, gerada pelo ideal das identidades dos quatérnios, que é o mesmo das matrizes $2 \times 2$, conforme Lema 4.5.2. Mas em $M_{2}(F)$ só existem identidades multilineares de grau 4. Assim, podemos nos restringir ao estudo das identidades dos dois últimos tipos, $[2,1,1]$ e a multilinear $[1,1,1,1]$.

\section{Identidades do tipo $[2,1,1]$}

Seja $f(x, y, z)$ uma identidade dos octônios, de grau 2 em $x$ e de grau 1 em $y$ e $z$. Pelas considerações acima, podemos assumir $f$ alternado em $y$ e $z$. Vamos mostrar que: (i) A imagem de $f$ em $A s s[X]$ é zero; (ii) $\nu f$ está no ideal associador de $A l t[X]$.

(i) $\phi f \in A s s[X] \Rightarrow \phi f=\alpha s_{4}, \quad \alpha \in F$. E como $s_{4}$ é multilinear, segue que $\alpha=0$ e $\phi f=0$.

(ii) $\nu f=f+J \Rightarrow \phi(\nu f)=\phi(f+J)=\phi f=0$.

Assim, como $\phi(\nu f)=0$, devemos considerar o homomorfismo canônico de $\operatorname{Alt}[X]$ para $A s s[X]$. A rigor, não é o mesmo $\phi: F[X] \rightarrow A s s[X]$, mas lembrando que a finalidade da $\phi$ é retirar os parêntesis, fica justificado o uso do mesmo símbolo.

Como $\nu f$ está no ideal associador de $A l t[X]$ deverá ser combinação linear de $\left(x^{2}, y, z\right),(x, x y, z)$ e $(x, y x, z)$. Mas, pelo Lema 4.5.3, podemos escrever:

$$
\left(x^{2}, y, z\right)=x \circ(x, y, z)=x(x, y, z)+(x, y, z) x=(x, y x, z)+(x, x y, z) .
$$

Portanto $\nu f$ é combinação linear de $x(x, y, z)$ e $(x, y, z) x$. Sendo $f$ uma identidade, $f(a, b, c)=0$, para todos $a, b, c \in \mathbb{O}$. Em particular, para elementos da base de $\mathbb{O}$. Lembramos que a base de $\mathbb{O}$, conforme estabelecemos no capítulo anterior, é dada por: 


$$
\left\{1, e_{1}, e_{2}, \ldots, e_{7}\right\}
$$

Façamos as seguintes substituições: $x=e_{1}, y=e_{2}, z=e_{3}$. Calculando, obtemos:

$$
\begin{aligned}
\nu f & =\alpha e_{1}\left(e_{1}, e_{2}, e_{3}\right)+\beta\left(e_{1}, e_{2}, e_{3}\right) e_{1} \\
& =\alpha e_{1}\left(\left(e_{1} e_{2}\right) e_{3}-e_{1}\left(e_{2} e_{3}\right)\right)+\beta\left(\left(e_{1} e_{2}\right) e_{3}-e_{1}\left(e_{2} e_{3}\right)\right) e_{1} \\
& =\alpha e_{1}\left(e_{3} e_{3}-e_{1} e_{1}\right)+\beta\left(e_{3} e_{3}-e_{1} e_{1}\right)=0
\end{aligned}
$$

Assim, $\nu f$ é o polinômio nulo. Provamos então que não existem identidades do tipo $[2,1,1]$ em $\operatorname{Alt}[X]$, além das triviais.

\section{Identidades do tipo $[1,1,1,1]$}

Para tratarmos deste tipo de polinômio precisamos determinar as possíveis formas de se arranjar parêntesis em 4 variáveis. Não é difícil perceber que tais arranjos se resumem nos seguintes, indicados esquematicamente:

$$
\begin{gathered}
s_{4}^{1}=\ldots\left(-\left(\_\_\right)\right) \quad s_{4}^{2}=\ldots\left(\left(\_\_\right) \_\right) \quad s_{4}^{3}=\left(\left(\_-\right)_{-}\right) \\
s_{4}^{4}=\left(\_\left(\_\_\right){ }_{-} \quad s_{4}^{5}=\left(\_\_\right)\left(\_\_\right)\right.
\end{gathered}
$$

É importante notar que, ao fazermos substituições por elementos da base dos octônios, não precisamos levar em conta todas as possibilidades de distribuição das variáveis em cada $s_{4}^{i}$, uma vez que os polinômios são alternados. Assim, uma troca de posição de duas variáveis afetará o monômio com o sinal da permutação. Por exemplo, no caso de $s_{4}^{1}$, teremos:

$$
e_{1}\left(e_{2}\left(e_{3} e_{4}\right)\right)=-e_{2}\left(e_{1}\left(e_{3} e_{4}\right)\right)=e_{2}\left(e_{1}\left(e_{4} e_{3}\right)\right)
$$

Basta portanto fazer uma única substituição por elementos da base. Então, notando que $f$ é combinação linear dos monômios $s_{4}^{i}$ indicados acima, temos:

$$
f=a s_{4}^{1}+b s_{4}^{2}+c s_{4}^{3}+d s_{4}^{4}+e s_{4}^{5}
$$

Fazendo as seguintes substituições:

$$
\begin{array}{ll}
\text { (1) } e_{1}, e_{2}, e_{3}, e_{4} ; & \text { (2) } e_{1}, e_{2}, e_{3}, e_{5} ; \\
\text { (3) } e_{1}, e_{2}, e_{3}, e_{6} ; & \text { (4) } e_{2}, e_{3}, e_{4}, e_{6}
\end{array}
$$

(5) $e_{2}, e_{3}, e_{4}, e_{7}$ 
obtemos um sistema homogêneo com 5 equações nas incógnitas $a, b, c, d, e$. Desenvolvendo:

(1) $a k_{1}+b k_{2}+c k_{3}+d k_{4}+e k_{5}=0$, onde

$$
\begin{aligned}
& k_{1}=e_{1}\left(e_{2}\left(e_{3} e_{4}\right)\right)=e_{1}\left(e_{2} e_{7}\right)=-e_{1} e_{5}=e_{4} \\
& k_{2}=e_{1}\left(\left(e_{2} e_{3}\right) e_{4}\right)=e_{1}\left(e_{1} e_{4}\right)=e_{1} e_{5}=-e_{4} \\
& k_{3}=\left(\left(e_{1} e_{2}\right) e_{3}\right) e_{4}=\left(e_{3} e_{3}\right) e_{4}=-e_{4} \\
& k_{4}=\left(e_{1}\left(e_{2} e_{3}\right)\right) e_{4}=\left(e_{1} e_{1}\right) e_{4}=-e_{4} \\
& k_{5}=\left(e_{1} e_{2}\right)\left(e_{3} e_{4}\right)=e_{3} e_{7}=-e_{4}
\end{aligned}
$$

A equação (1) fica: $(a-b-c-d-e) e_{4}=0$ ou $a-b-c-d-e=0$

(2) A equação (2) tem a mesma expressão acima, com as substituições agora por outros elementos, dando os seguintes resultados:

$$
\begin{aligned}
& k_{1}=e_{1}\left(e_{2}\left(e_{3} e_{5}\right)\right)=e_{1}\left(e_{2} e_{6}\right)=-e_{1} e_{4}=-e_{5} \\
& k_{2}=e_{1}\left(\left(e_{2} e_{3}\right) e_{5}\right)=e_{1}\left(e_{1} e_{5}\right)=-e_{1} e_{4}=-e_{5} \\
& k_{3}=\left(\left(e_{1} e_{2}\right) e_{3}\right) e_{5}=\left(e_{3} e_{3}\right) e_{5}=-e_{5} \\
& k_{4}=\left(e_{1}\left(e_{2} e_{3}\right)\right) e_{5}=\left(e_{1} e_{1}\right) e_{5}=-e_{5} \\
& k_{5}=\left(e_{1} e_{2}\right)\left(e_{3} e_{5}\right)=e_{3} e_{6}=e_{5}
\end{aligned}
$$

A equação (2) fica: $(-a-b-c-d+e) e_{5}=0$ ou $-a-b-c-d+e=0$

(3) De maneira análoga para a equação (3) obtemos:

$$
\begin{aligned}
& k_{1}=e_{1}\left(e_{2}\left(e_{3} e_{6}\right)\right)=e_{1}\left(e_{2} e_{5}\right)=e_{1} e_{7}=e_{6} \\
& k_{2}=e_{1}\left(\left(e_{2} e_{3}\right) e_{6}\right)=e_{1}\left(e_{1} e_{6}\right)=-e_{1} e_{7}=-e_{6} \\
& k_{3}=\left(\left(e_{1} e_{2}\right) e_{3}\right) e_{6}=\left(e_{3} e_{3}\right) e_{6}=-e_{6} \\
& k_{4}=\left(e_{1}\left(e_{2} e_{3}\right) e_{6}=\left(e_{1} e_{1}\right) e_{6}=-e_{6}\right. \\
& k_{5}=\left(e_{1} e_{2}\right)\left(e_{3} e_{6}\right)=e_{3} e_{5}=e_{6}
\end{aligned}
$$

A equação (3) fica: $(a-b-c-d+e) e_{6}=0$ ou $a-b-c-d+e=0$

(4) Agora, para a equação (4):

$$
\begin{aligned}
& k_{1}=e_{2}\left(e_{3}\left(e_{4} e_{6}\right)\right)=e_{2}\left(e_{3} e_{2}\right)=-e_{2} e_{1}=e_{3} \\
& k_{2}=e_{2}\left(\left(e_{3} e_{4}\right) e_{6}\right)=e_{2}\left(e_{7} e_{6}\right)=-e_{2} e_{1}=e_{3} \\
& k_{3}=\left(\left(e_{2} e_{3}\right) e_{4}\right) e_{6}=\left(e_{1} e_{4}\right) e_{6}=e_{5} e_{6}=-e_{3} \\
& k_{4}=\left(e_{2}\left(e_{3} e_{4}\right)\right) e_{6}=\left(e_{2} e_{7}\right) e_{6}=-e_{5} e_{6}=e_{3} \\
& k_{5}=\left(e_{2} e_{3}\right)\left(e_{4} e_{6}\right)=e_{1} e_{2}=e_{3}
\end{aligned}
$$


A equação (4) fica: $(a+b-c+d+e) e_{3}=0$ ou $a+b-c+d+e=0$

(5) Finalmente para a equação (5):

$$
\begin{aligned}
& k_{1}=e_{2}\left(e_{3}\left(e_{4} e_{7}\right)\right)=e_{2}\left(e_{3} e_{3}\right)=-e_{2} \\
& k_{2}=e_{2}\left(\left(e_{3} e_{4}\right) e_{7}\right)=e_{2}\left(e_{7} e_{7}\right)=-e_{2} \\
& k_{3}=\left(\left(e_{2} e_{3}\right) e_{4}\right) e_{7}=\left(e_{1} e_{4}\right) e_{7}=e_{5} e_{7}=-e_{2} \\
& k_{4}=\left(e_{2}\left(e_{3} e_{4}\right)\right) e_{7}=\left(e_{2} e_{7}\right) e_{7}=-e_{5} e_{7}=e_{2} \\
& k_{5}=\left(e_{2} e_{3}\right)\left(e_{4} e_{7}\right)=e_{1} e_{3}=-e_{2}
\end{aligned}
$$

A equação (5) fica: $(-a-b-c+d-e) e_{3}=0$ ou $-a-b-c+d-e=0$

Temos então o seguinte sistema:

$$
\begin{aligned}
a-b-c-d-e & =0 \\
-a-b-c-d+e & =0 \\
a-b-c-d+e & =0 \\
a+b-c+d+e & =0 \\
-a-b-c+d-e & =0
\end{aligned}
$$

que facilmente verificamos, só apresenta a solução trivial: $a=b=c=d=e=0$.

Portanto $f$ é o polinômio nulo. Concluímos assim que não existem identidades de grau 4 em uma álgebra de octônios, além das triviais.

\subsection{Identidades nos Octônios - Grau 5}

Mostraremos nesta seção que as identidades de grau 5 nos octônios são conseqüências de apenas duas identidades conhecidas, designadas por $\chi$ e $\kappa$, definidas da seguinte maneira:

$$
\begin{aligned}
& \chi=\left[\left[x_{1}, x_{2}\right] \circ\left[x_{3}, x_{4}\right], x_{5}\right] \\
& \kappa\left(x ; x_{1}, x_{2}, x_{3}\right)=s_{3}^{+}\left(x_{1}, x_{2}, x_{3}\right)\left(x^{2}\right)-\left(s_{3}^{+}\left(x_{1}, x_{2}, x_{3}\right)(x)\right) \circ x \\
& \kappa\left(x ; y ; x_{1}, x_{2}, x_{3}\right)=s_{3}^{+}(x \circ y)-\left(s_{3}^{+}(x)\right) \circ y-\left(s_{3}^{+}(y)\right) \circ x
\end{aligned}
$$

onde $s_{3}^{+}$é o operador definido por 


$$
s_{3}^{+}\left(x_{1}, x_{2}, x_{3}\right)(y)=s_{3}\left(V_{x_{1}}, V_{x_{2}}, V_{x_{3}}\right)(y)=\sum_{\pi \in S_{3}}(\operatorname{sgn} \pi) V_{x_{\pi_{1}}} V_{x_{\pi_{2}}} V_{x_{\pi_{3}}}(y)
$$

e $V_{x}$ é o produto de Jordan, dado por $V_{x}(y)=x \circ y=x y+y x$.

No que segue, adotaremos a seguinte simplificação na notação: Indicaremos $x_{\pi i}$ ao invés de $x_{\pi_{i}}$ para a permutação $\pi i$ do elemento $x$.

Lema 4.6.1. Os polinômios $\chi$ e $\kappa$ definidos acima são identidades nas álgebras de Cayley.

\section{Demonstração:}

(1) Provemos que $\chi$ é uma identidade em $C$.

Seja $C$ uma álgebra de Cayley. Então todo elemento $a \in C$ satisfaz uma equação quadrática do tipo $x^{2}-t(x) x+n(x) \cdot 1=0$, onde $t(x)$ é o traço de $x$ e $n(x)$ é a norma de $x$. Linearizando esta equação obtemos:

$$
x \circ y-t(x) y-t(y) x+n(x, y) \cdot 1=0
$$

Fazendo $x=[a, b]$ e $y=[c, d]$, nos dá:

$$
[a, b] \circ[c, d]-t([a, b])[c, d]-t([c, d])[a, b]+n([a, b],[c, d]) .1=0
$$

$\operatorname{Mas} t([a, b])=t([c, d])=0, \log 0: \chi(a, b, c, d, e)=[n([a, b],[c, d]) \cdot 1, e]=0$

(2) Provemos que $\kappa$ é uma identidade em $C^{+}$.

Obtemos a partir da álgebra $C$ dos octônios, uma álgebra de Jordan $C^{+}$, definindo o produto por $x \circ y$, conforme já indicado. Para maiores detalhes sobre a relação entre as álgebras alternativas e as álgebras de Jordan, ver referência [22].

Em $C^{+}$podemos considerar o traço $T(x)$ e a norma $Q(x)$ de um elemento e, de acordo com o que já estudamos no capítulo anterior, temos as seguintes igualdades:

$$
\begin{aligned}
& x^{2}=T(x)-Q(x) 1 ; \\
& Q(x, y)=q(x+y)-q(x)-q(y) ; \\
& T(x)=Q(x, 1) ; \\
& x \circ y=T(x) y+T(y) x-Q(x, y) 1
\end{aligned}
$$

Para mostrar (4) fazemos: 
$T(x) y+T(y) x-Q(x, y) 1=(x+\bar{x}) y+(y+\bar{y}) x-\bar{x} y-\bar{y} y=x y+y x$

Usando estas igualdades, reescrevemos $\kappa$ da seguinte maneira:

$\kappa\left(x ; x_{1}, x_{2}, x_{3}\right)=s_{3}^{+}(T(x) x-Q(x) 1)-\left(T\left(s_{3}^{+}(x)\right) x+T(x) s_{3}^{+}(x)-Q\left(s_{3}^{+}(x), x\right) 1\right)$

Vamos começar calculando a expressão $s_{3}^{+}(x)$, aplicando sua definição:

$$
\begin{aligned}
s_{3}^{+}(x) & =s_{3}\left(V_{x_{1}}, V_{x_{2}}, V_{x_{3}}\right)(x)=\sum_{\pi \in S_{3}}(\operatorname{sgn} \pi) V_{x_{\pi 3}} V_{x_{\pi 2}} V_{x_{\pi 3}}(x) \\
& =\sum_{\pi \in S_{3}}(\operatorname{sgn} \pi) V_{x_{\pi 3}} V_{x_{\pi 2}}\left(x_{\pi 1} \circ x\right) \\
& =\sum_{\pi \in S_{3}}(\operatorname{sgn} \pi) V_{x_{\pi 3}} V_{x_{\pi 2}}\left(T\left(x_{\pi 1}\right) x+T(x) x_{\pi 1}-Q\left(x_{\pi 1}, x\right) 1\right) \\
& =\sum_{\pi \in S_{3}}(\operatorname{sgn} \pi) V_{x_{\pi 3}}\left(x_{\pi 2} \circ\left(T\left(x_{\pi 1}\right) x+T(x) x_{\pi 1}-Q\left(x_{\pi 1}, x\right) 1\right)\right) \\
& =\sum_{\pi \in S_{3}}^{\pi}(\operatorname{sgn} \pi) V_{x_{\pi 3}}\left(x_{\pi 2} \circ T\left(x_{\pi 1}\right) x+x_{\pi 2} \circ T(x) x_{\pi 1}-x_{\pi 2} \circ Q\left(x_{\pi 1}, x\right) 1\right) \\
& =\sum_{\pi \in S_{3}}(\operatorname{sgn} \pi) V_{x_{\pi 3}} V_{1}+\sum_{\pi \in S_{3}}(\operatorname{sgn} \pi) V_{x_{\pi 3}} V_{2}-\sum_{\pi \in S_{3}}(\operatorname{sgn} \pi) V_{x_{\pi 3}} V_{3}
\end{aligned}
$$

onde $V_{1}=x_{\pi 2} \circ T\left(x_{\pi 1}\right) x, \quad V_{2}=x_{\pi 2} \circ T(x) x_{\pi 1}$ e $V_{3}=x_{\pi 2} \circ Q\left(x_{\pi 1}, x\right) 1$.

Analisemos cada uma destas parcelas separadamente. Comecemos pela segunda: $\sum_{\pi \in S_{3}}(\operatorname{sgn} \pi) V_{x_{\pi 3}} V_{2}=\sum_{\pi \in S_{3}}(\operatorname{sgn} \pi) x_{\pi 3} \circ\left(x_{\pi 2} \circ T(x) x_{\pi 1}\right)=T(x) \sum_{\pi \in S_{3}}(\operatorname{sgn} \pi) x_{\pi 3} \circ\left(x_{\pi 2} \circ x_{\pi 1}\right)$

Temos uma soma de elementos simétricos em $s_{3}$, uma vez que $x_{\pi 2} \circ x_{\pi 3}=x_{\pi 3} \circ x_{\pi 2}$. Mas, como $s_{3}$ é alternado estes dois termos têm sinais contrários, logo se anulam. Portanto:

$$
\sum_{\pi \in S_{3}}(\operatorname{sgn} \pi) V_{x_{\pi 3}} V_{2}=0 .
$$

Vejamos agora a terceira parcela.

$$
\begin{aligned}
\sum_{\pi \in S_{3}}(\operatorname{sgn} \pi) V_{x_{\pi 3}} V_{3} & =\sum_{\pi \in S_{3}}(\operatorname{sgn} \pi) x_{\pi 3} \circ\left(x_{\pi 2} \circ Q\left(x_{\pi 1}, x\right) 1\right) \\
& =2\left(Q\left(x_{1}, x\right) x_{3} \circ x_{2}-Q\left(x_{2}, x\right) x_{3} \circ x_{1}-Q\left(x_{1}, x\right) x_{2} \circ x_{3}\right. \\
& \left.+Q\left(x_{3}, x\right) x_{2} \circ x_{1}+Q\left(x_{2}, x\right) x_{1} \circ x_{3}-Q\left(x_{3}, x\right) x_{1} \circ x_{3}\right)
\end{aligned}
$$

Novamente temos uma soma de elementos simétricos que, pela alternatividade de $s_{3}$ (que agora explicitamos), resulta na nulidade da expressão. Assim:

$$
\sum_{\pi \in S_{3}}(\operatorname{sgn} \pi) V_{x_{\pi 3}} V_{3}=0
$$

Para a primeira parcela temos:

$$
\sum_{\pi \in S_{3}}(\operatorname{sgn} \pi) V_{x_{\pi 3}} V_{1}=\sum_{\pi \in S_{3}}(\operatorname{sgn} \pi) V_{x_{\pi 3}}\left(x_{\pi 2} \circ T\left(x_{\pi 1}\right) x\right)
$$




$$
\begin{aligned}
& \stackrel{(4)}{=} \sum_{\pi \in S_{3}}(\operatorname{sgn} \pi) V_{x_{\pi 3}}\left(T\left(x_{\pi 2}\right) T\left(x_{\pi 1}\right) x+T\left(T\left(x_{\pi 1}\right) x\right) x_{\pi 2}-Q\left(x_{\pi 2}, T\left(x_{\pi 1}\right) x\right) 1\right) \\
& =\sum_{\pi \in S_{3}}(\operatorname{sgn} \pi) V_{x_{\pi 3}}\left(T\left(x_{\pi 2}\right) T\left(x_{\pi 1}\right) x+T(x) T\left(x_{\pi 1}\right) x_{\pi 2}-T\left(x_{\pi 1}\right) Q\left(x_{\pi 2}, x\right) 1\right) \\
& =\sum_{\pi \in S_{3}}(\operatorname{sgn} \pi)(\underbrace{T\left(x_{\pi 1}\right) T\left(x_{\pi 2}\right)\left(x_{\pi 3} \circ x\right.}_{(i)}+\underbrace{T(x) T\left(x_{\pi 1}\right) x_{\pi 3} \circ x_{\pi 2}}_{(i i)}-2 T\left(x_{\pi 1}\right) Q\left(x_{\pi 2}, x\right) x_{\pi 3})
\end{aligned}
$$

Verifiquemos que as parcelas (i) e (ii) se anulam. De fato:

$$
\begin{aligned}
(\mathrm{i}) & =T\left(x_{1}\right) T\left(x_{2}\right)\left(x_{3} \circ x\right)-T\left(x_{1}\right) T\left(x_{3}\right)\left(x_{2} \circ x\right)-T\left(x_{2}\right) T\left(x_{1}\right)\left(x_{3} \circ x\right) \\
& +T\left(x_{2}\right) T\left(x_{3}\right)\left(x_{1} \circ x\right)+T\left(x_{3}\right) T\left(x_{1}\right)\left(x_{2} \circ x\right)-T\left(x_{3}\right) T\left(x_{2}\right)\left(x_{1} \circ x\right)=0
\end{aligned}
$$

De maneira análoga se mostra que a parcela (ii) também se anula.

Ficamos então com a seguinte expressão de $s_{3}^{+}$:

$$
s_{3}^{+}(x)=s_{3}\left(V_{x_{1}}, V_{x_{2}}, V_{x_{3}}\right)(x)=-2 \sum_{\pi \in S_{3}}(\operatorname{sgn} \pi) T\left(x_{\pi 1}\right) Q\left(x_{\pi 2}, x\right) x_{\pi 3}
$$

Vamos desenvolver a expressão de $\kappa$ obtida há pouco, usando a linearidade de $s_{3}^{+}$:

$$
\begin{gathered}
\kappa\left(x ; x_{1}, x_{2}, x_{3}\right)=s_{3}^{+}(T(x) x-Q(x) 1)-\left(T\left(s_{3}^{+}(x)\right) x+T(x) s_{3}^{+}(x)-Q\left(s_{3}^{+}(x), x\right) 1\right) \\
=T(x) s_{3}^{+}(x)-Q(x) s_{3}^{+}(1)-T\left(s_{3}^{+}(x)\right) x-T(x) s_{3}^{+}(x)+Q\left(s_{3}^{+}(x), x\right) 1 \\
\kappa\left(x ; x_{1}, x_{2}, x_{3}\right)=-Q(x) s_{3}^{+}(1)-T\left(s_{3}^{+}(x)\right) x+Q\left(s_{3}^{+}(x), x\right) 1
\end{gathered}
$$

Afirmamos que: $s_{3}^{+}(1)=0$. De fato, $s_{3}^{+}(1)=-2 \sum_{\pi \in S_{3}}(\operatorname{sgn} \pi) T\left(x_{\pi 1}\right) Q\left(x_{\pi 2}, 1\right) x_{\pi 3}=-2 \sum_{\pi \in S_{3}}(\operatorname{sgn} \pi) T\left(x_{\pi 1}\right) T\left(x_{\pi 2}\right) x_{\pi 3}=0$ pois temos uma soma alternada de elementos simétricos.

Verifiquemos que $Q\left(s_{3}^{+}(x), x\right)=0$.

$$
\begin{aligned}
Q\left(s_{3}^{+}(x), x\right) & =Q\left(-2 \sum_{\pi \in S_{3}}(\operatorname{sgn} \pi) T\left(x_{\pi 1}\right) Q\left(x_{\pi 2}, x\right) x_{\pi 3}, x\right) \\
& =-2 \sum_{\pi \in S_{3}}(\operatorname{sgn} \pi) T\left(x_{\pi 1}\right) Q\left(x_{\pi 2}, x\right) Q\left(x_{\pi 3}, x\right)=0
\end{aligned}
$$

pois temos também uma soma alternada de elementos simétricos.

Mostraremos agora que $T\left(s_{3}^{+}(x)\right)=0$. Para isto linearizamos $Q\left(s_{3}^{+}(x), x\right)=0$, substituindo $x$ por $x+1$ :

$$
\begin{aligned}
& 0=Q\left(s_{3}^{+}(x+1), x+1\right)=Q\left(s_{3}^{+}(x), x\right)+Q\left(s_{3}^{+}(x), 1\right)+Q\left(s_{3}^{+}(1), x\right)+Q\left(s_{3}^{+}(1), 1\right) \\
& \quad=\quad 0+T\left(s_{3}^{+}(x)\right)+0+0 \\
& \Rightarrow T\left(s_{3}^{+}(x)\right)=0 .
\end{aligned}
$$

Portanto $\kappa\left(x ; x_{1}, x_{2}, x_{3}\right)=0$. 
Teorema 4.6.2. O T-ideal das identidades em $M_{2}(F)$, sendo car $F=0$, é gerado por $s_{4}(x, y, z, w)$ e por $\left[[x, y]^{2}, x\right]$.

Demonstração: (idéia) Consiste em verificar a chamada conjectura de Razmyslov para $n=2$, segundo a qual, em uma álgebra associativa de matrizes de ordem $n$ sobre um corpo de característica zero, a base das identidades consiste das seguintes identidades:

$$
s_{2 n}\left(x_{1}, \ldots, x_{2 n}\right)=0 \text { e } s_{n}\left(\left[x_{1}^{n}, x_{2}\right],\left[x_{1}^{n-1}, x_{2}\right], \ldots,\left[x_{1}, x_{2}\right]\right)=0
$$

Esta conjectura é provada para $n=2$. Utiliza a teoria das representações do grupo simétrico. Ressalta o fato de que as identidades nas bases consideradas são de graus 4,5 e 6 . Os detalhes podem ser encontrados em [4].

Se $\operatorname{car} F \neq 2$, as identidades de grau 5 em $M_{2}(F)$ podem ser de um dos seguintes tipos:

$$
s_{4}(\ldots, \ldots, \ldots, \ldots) \quad s_{4}(\ldots, \ldots, \ldots, \ldots) s_{4}(\ldots, \ldots, \ldots, \ldots) \quad[[\ldots, \ldots] \circ[\ldots, \ldots], \ldots]
$$

Para tratarmos o caso das identidades de grau 5, são válidas as mesmas simplificações assumidas no grau 4, isto é, que basta considerar polinômios homogêneos e alternados.

Um polinômio homogêneo do grau 5 pode ser obtido a partir das partições de 5 , que são: $[5],[4,1],[3,2],[3,1,1],[2,2,1],[2,1,1,1]$ e $[1,1,1,1,1]$. Daqui para frente, ao invés de nos referirmos a um polinômio (ou identidade) de grau 5 , vamos falar de polinômio de grau [5] ou de grau [4,1], etc, enfatizando a partição em estudo.

As três primeiras partições resultam em polinômios associativos, pois têm apenas 1 ou 2 variáveis, logo pelo Teorema de Artin, geram uma subálgebra associativa, isto é, pertencem a $M_{2}(F)$. É fácil ver que não existem identidades dos dois primeiros tipos em $M_{2}(F)$. Se houver uma identidade de grau [3,2] em $M_{2}(F)$, ela é múltipla de $\left[[x, y]^{2}, x\right]$. Nosso estudo das identidades de grau 5 se resumirá, portanto, às identidades de grau $[3,1,1],[2,2,1],[2,1,1,1]$ e $[1,1,1,1,1]$.

Interessa agora explicitar os geradores de cada um desses graus, com base no Teorema 4.6.2. Mostraremos a seguir que as identidades de grau $[3,1,1]$ são conseqüência apenas de $s_{4}$, e que as identidades de grau $[2,2,1]$ são conseqüência de $s_{4}$ e de $\chi$. 
Lema 4.6.3. Sejam $f$ uma identidade de grau $[3,1,1]$ e g uma identidade de grau $[2,2,1]$. A imagem de $f$ em Ass[X], $\phi f$, é conseqüência de $s_{4}$ e a imagem de $g$ em Ass $[X], \phi g$, é conseqüência de $\chi$.

Demonstração: Linearizando $\left[[x, y]^{2}, x\right]$ para 3 variáveis, temos:

$$
\begin{aligned}
{\left[[x+z, y]^{2}, x+z\right] } & =\left[([x, y]+[z, y])^{2}, x+z\right] \\
& =\left[[x, y]^{2}, x\right]+\left[[z, y]^{2}, x\right]+\left[[x, y]^{2}, z\right]+\left[[z, y]^{2}, z\right] \\
& +[[x, y] \circ[z, y], x]+[[x, y] \circ[z, y], z]
\end{aligned}
$$

Os elementos acima obtidos são de graus: $[3,2,0],[1,2,2],[2,2,1],[0,2,3],[2,2,1]$ e $[1,2,2]$, respectivamente. Portanto, dentre os dois casos em estudo, vemos que somente o de grau $[2,2,1]$ é conseqüência de $\chi$. Mais especificamente, temos que uma identidade de grau $[2,2,1]$ é conseqüência de $\left[[x, y]^{2}, z\right]$ e de $[[x, y] \circ[z, y], x]$.

Por outro lado, uma identidade de grau $[3,1,1]$ pode ser obtida a partir de $s_{4}\left(x, y, z, x^{2}\right)$, que é um dos cinco tipos possíveis para uma identidade associativa de grau 5 , a saber, $s_{4}(\ldots, \ldots, \ldots, \ldots)$. Podemos ter um polinômio de grau $[3,1,1]$ do tipo $[[x, y] \circ[x, z], x]$, mas este é um polinômio simétrico em $y$ e $z$. Como estamos supondo identidades alternadas nas variáveis lineares, um polinômio deste tipo só pode ser o polinômio nulo.

Pelo mesmo argumento, temos que uma identidade de grau $[2,2,1]$ é gerada somente por $s_{4}(x, y, z, x y)$ e $s_{4}(x, y, z, y x)$, pois não há outra distribuição das variáveis em $s_{4}$ que resulte no grau em questão.

Veremos adiante que também as identidades de grau $[2,1,1,1]$ são conseqüência de $\chi$ e de $s_{4}$. Mas queremos mostrar que qualquer identidade de grau 5 é conseqüência de $\chi$ e de $\kappa$. Precisamos, então, relacionar $s_{4} \operatorname{com} \kappa$. Para isto, o conceito de derivação tem importância.

Definição 4.6.1. Seja A uma álgebra sobre um corpo K. Chama-se derivação de $A$ a um operador linear $D$ que satisfaz:

$$
D(x y)=(D(x)) y+x(D(y))
$$

Como exemplo, citamos: Em uma álgebra alternativa, $D=\left(x, y, \_\right)$é uma derivação. De fato, se $D\left(z^{2}\right)=D(z z)=z D(z)+(D(z)) z$, então, de acordo com o Lema 4.5.3 (iii), $\left(x, y, z^{2}\right)=z(x, y, z)+(x, y, z) z$. 
A proposição a seguir relaciona $s_{4} \operatorname{com} \kappa$.

Proposição 4.6.4. Em uma álgebra associativa vale a seguinte igualdade:

$$
\kappa(x ; y, z, w)=s_{4}\left(y, z, w, x^{2}\right)-s_{4}(y, z, w, x) \circ x
$$

Demonstração: Substituindo a expressão de $\kappa$ pela sua definição, trocando $x_{1}$ por $y, x_{2}$ por $z$ e $x_{3}$ por $w$, temos:

$\kappa(x ; y, z, w)=s_{3}^{+}(y, z, w)\left(x^{2}\right)-\left(s_{3}^{+}(y, z, w)(x)\right) \circ x$

Provar a tese equivale a mostrar que:

$s_{3}^{+}(y, z, w)\left(x^{2}\right)-\left(s_{3}^{+}(y, z, w)(x)\right) \circ x=s_{4}\left(y, z, w, x^{2}\right)-s_{4}(y, z, w, x) \circ x$

$\Rightarrow s_{3}^{+}(y, z, w)\left(x^{2}\right)-s_{4}\left(y, z, w, x^{2}\right)=\left(s_{3}^{+}(y, z, w)(x)-s_{4}(y, z, w, x)\right) \circ x$

Por sua vez, a validade desta última expressão equivale a mostrar que:

$s_{3}^{+}(y, z, w)(x)-s_{4}(y, z, w, x)$ é uma derivação em qualquer álgebra associativa.

Façamos $D_{y, z, w}(x)=s_{3}^{+}(y, z, w)(x)-s_{4}(y, z, w, x)$. Desenvolvendo cada parcela:

$$
\begin{aligned}
s_{3}^{+}(y, z, w)(x)= & s_{3}\left(V_{y}, V_{z}, V_{w}\right)(x) \\
= & V_{y} V_{z} V_{w}(x)-V_{y} V_{w} V_{z}(x)-V_{z} V_{y} V_{w}(x) \\
= & V_{z} V_{w} V_{y}(x)+V_{w} V_{y} V_{z}(x)-V_{w} V_{z} V_{y}(x) \\
= & V_{z} V_{w}(y \circ x)-V_{w} V_{z}(y \circ x)+V_{w} V_{y}(z \circ x)-V_{y} V_{w}(z \circ x) \\
& +V_{y} V_{z}(w \circ x)-V_{z} V_{y}(w \circ x) \\
= & {\left[V_{z}, V_{w}\right](y \circ x)+\left[V_{w}, V_{y}\right](z \circ x)+\left[V_{y}, V_{z}\right](w \circ x) } \\
= & \sum_{(y, z, w)}\left[V_{y}, V_{z}\right](x \circ y)
\end{aligned}
$$

onde a notação $\sum_{(y, z, w)}$ indica a soma cíclica em $y, z, w$.

No desenvolvimento de $s_{4}(y, z, w, x)$ existirão parcelas em que o $x$ será o último elemento, as quais poderão ser escritas como $s_{3}(y, z, w) x$. Teremos também termos em que o $x$ será o primeiro elemento, que escreveremos $x s_{3}(y, z, w)$.

Teremos ainda parcelas com o $x$ aparecendo em segundo lugar, como $y x z w$, as quais serão indicadas por $\sum_{(y, z, w)} w x[y, z]$.

Finalmente, quando o $x$ ocupar o terceiro lugar, como em $y z x w$, estes termos se escreverão: $-\sum_{(y, z, w)}[y, z] x w$. Temos então: 


$$
\begin{aligned}
D_{y, z, w}(x) & =\sum_{(y, z, w)}\left[V_{y}, V_{z}\right](x \circ y)-s_{3}(y, z, w) x+x s_{3}(y, z, w) \\
& +\sum_{(y, z, w)}[y, z] x w-\sum_{(y, z, w)} w x[y, z]
\end{aligned}
$$

Mas

$$
\begin{aligned}
& \sum_{(y, z, w)}\left[V_{y}, V_{z}\right](x \circ y)=\sum_{(y, z, w)}\left(V_{z} V_{w}(x \circ y)-V_{w} V_{z}(x \circ y)\right) \\
& =\sum_{(y, z, w)}\left(V_{w}(z \circ(x \circ y))-V_{z}(w \circ(x \circ y))\right) \\
& =\sum_{(y, z, w)}(w z x y+w z y x+w x y z+w y x z+z x y w+z y x w \\
& +x y z w+y x z w-z w x y-z w y x-z x y w-z y x w \\
& -w x y z-w y x z-x y w z-y x w z) \\
& =\sum_{(y, z, w)}[x y+y x,[z, w]]
\end{aligned}
$$

Resulta então:

$$
\begin{aligned}
D_{y, z, w}(x) & =\sum_{(y, z, w)}[x y+y x,[z, w]]+\left[x, s_{3}(y, z, w)\right]+\sum_{(y, z, w)}[z, w] x y-\sum_{(y, z, w)} y x[z, w] \\
& =\left[x, s_{3}(y, z, w)\right]+\sum_{(y, z, w)}([x y,[z, w]]+[y x,[z, w]]+[z, w] x y-y x[z, w]) \\
& =\left[x, s_{3}(y, z, w)\right] \\
& +\sum_{(y, z, w)}(x y[z, w]-[z, w] x y+y x[z, w]-[z, w] y x+[z, w] x y-y x[z, w]) \\
& =\left[x, s_{3}(y, z, w)\right]+\sum_{(y, z, w)}(x y[z, w]-[z, w] y x) \\
& =\left[x, s_{3}(y, z, w)\right]+x\left(\sum_{(y, z, w)} y[z, w]\right)-\left(\sum_{(y, z, w)}[z, w] y\right) x
\end{aligned}
$$

Mas

$$
\begin{aligned}
x\left(\sum_{(y, z, w)} y[z, w]\right)-\left(\sum_{(y, z, w)}[z, w] y\right) x & =x(y[z, w]+w[y, z]+z[w, y]) \\
& =x(y z w-y w z+w y z-w z y+z w y-z y w) \\
& -(z w y-w z y+y z w-z y w+w y z-y w z) x \\
& =x s_{3}(y, z, w)-s_{3}(y, z, w) x=\left[x, s_{3}(y, z, w)\right] \\
\Rightarrow D_{y, z, w}(x)=2\left[x, s_{3}(y, z, w)\right]= & {\left[x, 2 s_{3}(y, z, w)\right] . }
\end{aligned}
$$

Isto mostra que $D_{y, z, w}(x)$ é uma derivação, pois em uma álgebra associativa, $D_{z}(x)=[x, z]$ é uma derivação. De fato:

$$
\begin{aligned}
D_{z}(x y)=[x y, z] & =(x y) z-z(x y)=x y z-z x y . \\
D_{z}(x) y+x D_{z}(y) & =[x, z] y+x[y, z]=(x z-z x) y+x(y z-z y) \\
& =x z y-z x y+x y z-x z y=x y z-z x y
\end{aligned}
$$


Identidades de grau $[3,1,1]$

Seja $f(x, y, z)$ uma identidade dos octônios, de grau [3,1,1] alternada em $y$ e z. Considerando o homomorfismo canônico $\phi$ já definido, que leva uma identidade $f$ de $A l t[X]$ em $A s s[X]$, temos que $\phi f \in A s s[X]$, logo $\phi f$ é uma identidade em $M_{2}(F)$. Uma identidade de grau $[3,1,1]$ deve ter sua imagem em Ass[X] múltipla de $s_{4}\left(x, y, z, x^{2}\right)$, de acordo com o Lema 4.6.3. Escrevemos então, para $\lambda \in F$ :

$$
\phi f=\lambda s_{4}\left(x, y, z, x^{2}\right)
$$

Fazendo $y=x, z=y, w=z$ na Proposição 4.6.4, teremos: $\kappa(x ; x, y, z)=$ $s_{4}\left(x, y, z, x^{2}\right)$, uma vez que $s_{4}$ é alternado. Substituindo este dado em 4.1, temos: $\phi f=\lambda \kappa$.

Se $f$ e $\lambda \kappa$ são identidades, então $f^{\prime}=f-\lambda \kappa$ também é. Daí:

$\phi f^{\prime}=\phi(f-\lambda \kappa)=\phi f-\phi(\lambda \kappa)=\phi f-\phi f=0$ e $f^{\prime}$ está no ideal associador. Então $f^{\prime}$ é combinação linear de $x^{2}(x, y, z), x(x, y, z) x$ e $(x, y, z) x^{2}$. Assim:

$$
f^{\prime}=a x^{2}(x, y, z)+b x(x, y, z) x+c(x, y, z) x^{2}
$$

Vamos fazer as seguintes substituições por elementos da base de $C$ :

(1) $x=e_{1}, \quad y=e_{2}, \quad z=e_{4}$

$$
\begin{aligned}
& \left(e_{1}, e_{2}, e_{4}\right)=\left(e_{1} e_{2}\right) e_{4}-e_{1}\left(e_{2} e_{4}\right)=e_{3} e_{4}-e_{1} e_{6}=e_{7}-\left(-e_{7}\right)=2 e_{7} \\
& f^{\prime}=-2 a e_{7}+b e_{1}\left(2 e_{7}\right) e_{1}-2 c e_{7}=(-2 a+2 b-2 c) e_{7}
\end{aligned}
$$

(2) $x=1+e_{1}, \quad y=e_{2}, \quad z=e_{4}$

$$
\begin{aligned}
& \left(1+e_{1}, e_{2}, e_{4}\right)=\left(1, e_{2}, e_{4}\right)+\left(e_{1}, e_{2}, e_{4}\right)=0+2 e_{7}=2 e_{7} \\
& \left(1+e_{1}\right)^{2}=1+2 e_{1}+e_{1}^{2}=1+2 e_{1}-1=2 e_{1} \\
& f^{\prime}=-2 a e_{1}\left(2 e_{7}\right)+2 b e_{1}\left(2 e_{7}\right)+2 c e_{1}\left(2 e_{7}\right)=(4 a+4 b+4 c) e_{6}
\end{aligned}
$$

(3) $x=e_{1}, \quad y=e_{2}, \quad z=e_{6}$

$$
\begin{aligned}
& \left(e_{1}, e_{2}, e_{6}\right)=\left(e_{1} e_{2}\right) e_{6}-e_{1}\left(e_{2} e_{6}\right)=e_{3} e_{6}-e_{1}\left(-e_{4}\right)=e_{5}+e_{5}=2 e_{5} \\
& f^{\prime}=-2 a\left(2 e_{5}\right)-2 b e_{5}-2 c e_{5}=(-4 a-2 b-2 c) e_{5}
\end{aligned}
$$

Obtemos o sistema:

$$
\begin{array}{r}
-a+b-c=0 \\
a+b+c=0 \\
2 a+b+c=0
\end{array}
$$


que facilmente verificamos, só apresenta a solução trivial: $a=b=c=0$. Portanto $f^{\prime}$ é o polinômio nulo.

\section{Identidades de grau $[2,2,1]$}

Neste caso formaremos, a partir de uma identidade do tipo $[2,2,1]$, duas outras identidades, sendo uma simétrica e a outra alternada. Seja $f(x, y, z)$ uma identidade do tipo $[2,2,1]$. Então

1. $g(x, y, z)=f(x, y, z)+f(y, x, z)$ é simétrico em $x$ e $y$. De fato, $g(y, x, z)=$ $f(y, x, z)+f(x, y, z)=g(x, y, z)$

2. $h(x, y, z)=f(x, y, z)-f(y, x, z)$ é alternado em $x$ e $y$. De fato, $h(y, x, z)=$ $f(y, x, z)-f(x, y, z)=-h(x, y, z)$

Basta provar que $g$ e $h$ são conseqüências de $\chi$ e $\kappa$, uma vez que $f=\frac{g+h}{2}$. Sabemos, pelo Lema 4.6.3, que uma identidade de grau $[2,2,1]$ é conseqüência de $s_{4}(x, y, z, x y)$, de $s_{4}(x, y, z, y x)$ e de $\chi$. Mas não temos uma relação entre $s_{4}$ com estas distribuições de variáveis e $\kappa$. Vamos mostrar que, na verdade, estes $s_{4}$ estão relacionados com $\chi$ no caso simétrico e com $\kappa$ no caso alternado. Para isto precisaremos de um lema operacional.

Lema 4.6.5. Em Ass $[X]$ são válidas as seguintes igualdades:

(i) $s_{4}(x, y, z, w)=[x, y] \circ[z, w]+[x, z] \circ[w, y]+[x, w] \circ[y, z]$;

(ii) $[x, y] \circ z=[x, y \circ z]-y \circ[x, z]$

(iii) $[[x, y], z]+[[y, z], x]+[[z, x], y]=0$ (identidade de Jacobi)

Demonstração: As três igualdades são de verificação imediata, bastando apenas desenvolver os comutadores.

Teorema 4.6.6. O subespaço dos elementos simétricos de grau $[2,2,1]$ no ideal das identidades de grau 5 é gerado por $\left[z,[x, y]^{2}\right],[x,[x, y] \circ[y, z]]$ e $[y,[x, y] \circ[x, z]]$.

Demonstração: Pelo Lema 4.6.3, sabemos que uma identidade de grau $[2,2,1]$ é conseqüência de $\left[[x, y]^{2}, z\right]$, de $[[x, y] \circ[z, y], x]$, de $s_{4}(x, y, z, x y)$ e de $s_{4}(x, y, z, y x)$. O primeiro elemento é simétrico em $x$ e $y$, pois: 


$$
\left[[x, y]^{2}, z\right]=\left[(x y-y x)^{2}, z\right]=\left[(y x-x y)^{2}, z\right]=\left[[y, x]^{2}, z\right]
$$

O elemento $[[x, y] \circ[z, y], x]$ não é simétrico, mas notando que:

$$
[[x, y] \circ[z, y], x]=[x,[x, y] \circ[y, z]]
$$

podemos fazer:

$$
\begin{aligned}
p(x, y, z) & =[x,[x, y] \circ[y, z]]-[y,[x, y] \circ[x, z]] \\
& =-[x,[y, x] \circ[y, z]]+[y,[y, x] \circ[x, z]] \\
& =p(y, x, z)
\end{aligned}
$$

obtendo o elemento simétrico $p(x, y, z)=[x,[x, y] \circ[y, z]]-[y,[x, y] \circ[x, z]]$.

Os outros elementos de grau $[2,2,1]$, provenientes de $s_{4}(x, y, z, x y)$ e $s_{4}(x, y, z, y x)$, podem ser escritos na forma $s_{4}(x, y, z,[x, y])$, para os elementos simétricos. Vamos mostrar agora que este elemento é consequência de $\left[z,[x, y]^{2}\right]$ e $p(x, y, z)$. Pelo Lema 4.6.5 (i):

$$
s_{4}(x, y, z,[x, y])=\underbrace{[x, y] \circ[z,[x, y]]}_{(1)}+\underbrace{[x, z] \circ[[x, y], y]}_{(2)}+\underbrace{[x,[x, y]] \circ[y, z]}_{(3)}
$$

Aplicando a cada uma das 3 parcelas à direita o Lema 4.6 .5 (ii), temos:

$$
\begin{aligned}
{[x, y] \circ[z,[x, y]] } & =[z,[x, y]] \circ[x, y]=\left[z, 2[x, y]^{2}\right]-[x, y] \circ[z,[x, y]] \\
& =2\left[z,[x, y]^{2}\right]-[z,[x, y]] \circ[x, y] \\
& \Rightarrow 2[z,[x, y]] \circ[x, y]=2\left[z,[x, y]^{2}\right] \\
& \Rightarrow[z,[x, y]] \circ[x, y]=\left[z,[x, y]^{2}\right]
\end{aligned}
$$

$(2): \quad[x, z] \circ[[x, y], y]=[x, z] \circ[y,[y, x]]=[y,[y, x] \circ[x, z]]-[y, x] \circ[y,[x, z]]$

$$
=-[y,[x, y] \circ[x, z]]+[x, y] \circ[y,[x, z]]
$$

(3): $\quad[x,[x, y]] \circ[y, z]=[x,[x, y] \circ[y, z]]-[x, y] \circ[x,[y, z]]$

Reescrevendo a expressão de $s_{4}$ :

$$
\begin{aligned}
s_{4}(x, y, z,[x, y]) & =[x, y] \circ[z,[x, y]]+[x, y] \circ[y,[x, z]]-[x, y] \circ[x,[y, z]] \\
& +[x,[x, y] \circ[y, z]]-[y,[x, y] \circ[x, z]]
\end{aligned}
$$


A parcela (1) pode ser escrita:

$$
\begin{aligned}
{[x, y] \circ[z,[x, y]] } & =2([x, y] \circ[z,[x, y]])-[x, y] \circ[z,[x, y]] \\
& =2\left[z,[x, y]^{2}\right]+[x, y] \circ[z,[y, x]]
\end{aligned}
$$

Logo:

$$
\begin{aligned}
s_{4}(x, y, z,[x, y]) & =2\left[z,[x, y]^{2}\right]+[x, y] \circ \overbrace{([z,[y, x]]+[y,[x, z]]+[x,[z, y]])}^{=0(\text { Jacobi })} \\
& +[x,[x, y] \circ[y, z]]-[y,[x, y] \circ[x, z]] \\
\Rightarrow \quad s_{4}(x, y, z,[x, y]) & =2\left[z,[x, y]^{2}\right]+[x,[x, y] \circ[y, z]]-[y,[x, y] \circ[x, z]]
\end{aligned}
$$

como queríamos.

Proposição 4.6.7. Em uma álgebra de Cayley tem-se:

$$
\kappa(x ; y ; z, v, w)=s_{4}(z, v, w, x \circ y)-x \circ s_{4}(z, v, w, y)-y \circ s_{4}(z, v, w, x)
$$

Demonstração: Lembrando a conclusão da Proposição 4.6.4, tivemos:

$s_{3}^{+}(y, z, w)(x)-s_{4}(y, z, w, x)=\left[x, 2 s_{3}(y, z, w)\right]$

Fazendo $y=z, z=v$, e pondo $x \circ y$ no lugar do argumento $x$, teremos:

$s_{3}^{+}(z, v, w)(x \circ y)-s_{4}(z, v, w, x \circ y)=2(x \circ y) s_{3}-2 s_{3}(x \circ y)$

$\Rightarrow s_{3}^{+}(x \circ y)=s_{4}(z, v, w, x \circ y)+2(x \circ y) s_{3}-2 s_{3}(x \circ y)$

Voltando à forma original da expressão obtida na mesma proposição, calculemos:

$\left(s_{3}^{+}(x)\right) \circ y=s_{4}(z, v, w, x) \circ y+2\left(x s_{3}\right) \circ y-2\left(s_{3} x\right) \circ y$

$\left(s_{3}^{+}(y)\right) \circ x=s_{4}(z, v, w, y) \circ x+2\left(y s_{3}\right) \circ x-2\left(s_{3} y\right) \circ x$

Fazendo (1) - (2) - (3) teremos no primeiro membro a expressão de definição de $\kappa$. No segundo membro temos os termos desejados mais as seguintes parcelas, que mostraremos que se anulam:

$$
\begin{aligned}
& 2(x \circ y) s_{3}-2 s_{3}(x \circ y)-2\left(x s_{3}\right) \circ y+2\left(s_{3} x\right) \circ y-2\left(y s_{3}\right) \circ x+2\left(s_{3} y\right) \circ x \\
= & 2\left(x y s_{3}+y x s_{3}-s_{3} x y-s_{3} y x-x s_{3} y-y x s_{3}+s_{3} x y+y s_{3} x-y s_{3} x-x y s_{3}+s_{3} y x+x s_{3} y\right)=0
\end{aligned}
$$

Corolário 4.6.8. Em uma álgebra de Cayley tem-se:

$$
\kappa(x, y ; x, y, z)=s_{4}(x, y, z, x \circ y)
$$


Demonstração: É imediato, bastando fazer $z=x, v=y$ e $w=z$. Teremos:

$$
\begin{aligned}
& \kappa(x, y ; x, y, z)=s_{4}(x, y, z, x \circ y)-x \circ s_{4}(x, y, z, y)-y \circ s_{4}(x, y, z, x) \\
& \Rightarrow \kappa(x, y ; x, y, z)=s_{4}(x, y, z, x \circ y) .
\end{aligned}
$$

Teorema 4.6.9. O subespaço dos elementos alternados em $x$ e y de grau $[2,2,1]$ no ideal $I$ das identidades de grau 5 é gerado por $s_{4}(x, y, z, x \circ y)$ e $q(x, y, z)=$ $[x,[x, y] \circ[y, z]]+[y,[x, y] \circ[x, z]]$.

Demonstração: A argumentação é inteiramente análoga a do caso simétrico.

Mostramos assim que $g$ e $h$ são conseqüências de $\chi$ e $\kappa$, logo, $f$ também é. Qualquer outra identidade obtida de $f$ deve pertencer ao ideal associador dos elementos de grau $[2,2,1]$. Um elemento qualquer deste ideal associador é uma combinação linear de $(x y) a,(y x) a,(x a) y,(a x) y,(y a) x$ e $(a y) x$, onde $a=(x, y, z)$. Vamos mostrar que, neste caso, $f=0$. Mas antes, cabe esclarecer porque não precisamos dos termos com parêntesis à direita no ideal associador, como $x(y a), x(a y)$, etc. Para isto iremos demonstrar um lema que estabelece importantes relações entre o associador e o comutador em uma álgebra alternativa.

Lema 4.6.10. Em uma álgebra alternativa $A$, são válidas as seguintes igualdades, para todos $x, y, z \in A$ :

(i) $(x, y,(x, y, z))=[x, y](x, y, z)$;

(ii) $((x, y, z), x, y)=-(x, y, z)[x, y]$;

(iii) $[x, y] \circ(x, y, z)=0$;

(iv) $[x,(y, z, w)]=(x, y, z w)+(x, z, w y)+(x, w, y z)$.

\section{Demonstração:}

(i) Vamos provar antes a seguinte identidade, válida para álgebras alternativas: $(x y)(x, y, z)=y(x(x, y, z))$. Devemos linearizar a identidade (i) de Moufang, $(x y x) z=x(y(x z)), \operatorname{com} x \rightarrow x+w:$

$$
\begin{aligned}
& ((x+w) y(x+w)) z=(x+w)(y((x+w) z)) \\
\Rightarrow \quad & ((x y+w y)(x+w)) z=(x+w)(y(x z+w z))
\end{aligned}
$$




$$
\begin{aligned}
& \Rightarrow(x y x+(x y) w+(w y) x+w y w) z=(x+w)(y(x z)+y(w z)) \\
& \Rightarrow \quad(x y x) z+((x y) w) z+((w y) x) z+(w y w) z \\
& \quad=x(y(x z))+x(y(w z))+w(y(x z))+w(y(w z)) \\
& \Rightarrow((x y) w) z+((w y) x) z=x(y(w z))+w(y(x z))
\end{aligned}
$$

Fazendo $w=x y, \quad x=y$ e $y=x$, obtemos:

$((y x)(x y)) z+((x y x) y) z=y(x((x y) z))+(x y)(x(y z))$

Note que: $(x y x) y=(x y)^{2}$. De fato:

$(x y x) y-(x y)^{2}=((x y) x) y-(x y)(x y)=(x y, x, y)=0, \quad$ por 4.5 .3 (i).

Ficamos então com: $((y x)(x y)) z+(x y)^{2} z=y(x((x y) z))+(x y)(x(y z))$

Calculamos agora: $(x y)(x, y, z)-y(x(x, y, z))$

$$
\begin{aligned}
& =(x y)((x y) z-x(y z))-y(x((x y) z-x(y z))) \\
& =(x y)(x y) z-(x y)(x(y z))-y(x((x y) z)-x(x(y z))) \\
& =(x y)^{2} z-(x y)(x(y z))-y(x((x y) z))+y\left(x^{2}(y z)\right) \\
& \stackrel{(I)}{=}(x y)^{2} z-((y x)(x y)) z-(x y)^{2} z+y\left(x^{2}(y z)\right) \stackrel{*}{=}-\left(y x^{2} y\right) z+\left(y x^{2} y\right) z=0
\end{aligned}
$$

(*) Moufang (i) e (ii)

Calculamos, finalmente:

$$
\begin{aligned}
(x, y,(x, y, z)) & =-(y, x,(x, y, z))=y(x(x, y, z))-(y x)(x, y, z) \\
& =(x y)(x, y, z)-(y x)(x, y, z)=[x, y](x, y, z)
\end{aligned}
$$

(ii) De maneira análoga ao caso anterior, provemos primeiro que, em uma álgebra alternativa vale: $(x, y, z)(x y)=((x, y, z) y) x$. Vamos agora linearizar Moufang (ii), $(x y)(z x)=x(y z) x, \operatorname{com} x \rightarrow x+w$ :

$$
\begin{aligned}
& ((x+w) y)(z(x+w))=(x+w)(y z)(x+w) \\
\Rightarrow & (x y+w y)(z x+z w)=(x(y z)+w(y z))(x+w) \\
\Rightarrow & (x y)(z x)+(x y)(z w)+(w y)(z x)+(w y)(z w) \\
= & x(y z) x+(x(y z)) w+(w(y z)) x+(w(y z)) w \\
\Rightarrow & (x y)(z w)+(w y)(z x)=(x(y z)) w+(w(y z)) x
\end{aligned}
$$




$$
\Rightarrow(x y)(z w)-(x(y z)) w=(w(y z)) x-(w y)(z x)
$$

Fazendo $w=x y$, obtemos:

$$
\begin{aligned}
(x y)(z(x y))-(x(y z))(x y) & =((x y)(y z)) x-((x y) y)(z x) \\
& =((x y)(y z)) x-x\left(y^{2} z\right) x
\end{aligned}
$$

Calculamos agora: $(x, y, z)(x y)-((x, y, z) y) x$

$$
\begin{aligned}
& =((x y) z-x(y z))(x y)-(((x y) z-x(y z)) y) x \\
& =(x y) z(x y)-(x(y z))(x y)-(((x y) z) y) x+((x(y z)) y) x \\
& \stackrel{(I I)}{=}((x y)(y z)) x-x\left(y^{2} z\right) x-x(y z y) x+((x(y z)) y) x \\
& =\left[(x y)(y z)-x\left(y^{2} z\right)-x(y z y)+(x(y z)) y\right] x \\
& =[(x y)(y z)-x(y(y z))+(x(y z)) y-x((y z) y)] x \\
& =[(x, y, y z)+(x, y z, y)] x=0
\end{aligned}
$$

Calculamos finalmente:

$$
\begin{aligned}
((x, y, z), x, y) & =-((x, y, z), y, x)=(x, y, z)(y x)-((x, y, z) y) x \\
& =(x, y, z)(y x)-(x, y, z)(x y)=-(x, y, z)[x, y]
\end{aligned}
$$

(iii) Usando as igualdades que acabamos de provar, calculamos:

$$
\begin{aligned}
{[x, y] \circ(x, y, z) } & =[x, y](x, y, z)+(x, y, z)[x, y] \\
& =(x, y,(x, y, z))-((x, y, z), x, y)=0
\end{aligned}
$$

(iv) Como em (ii), vamos usar a linearização da identidade (ii) de Moufang,

$(x y)(z x)=x(y z) x$, onde obtivemos:

$(x y)(z w)-(x(y z)) w=(w(y z)) x-(w y)(z x)$

Calculamos agora:

$$
\begin{aligned}
x(y, z, w) & =x((y z) w)-x(y(z w)) \\
& =-(x, y z, w)+(x(y z)) w+(x, y, z w)-(x y)(z w) \\
& \stackrel{(I I I)}{=}-(x, y z, w)+(x, y, z w)+(w y)(z x)-(w(y z)) x \\
& =-(x, y z, w)+(x, y, z w)+(w, y, z) x-((w y) z) x+(w y)(z x) \\
& =(x, w, y z)+(x, y, z w)+(w, y, z) x-(w y, z, x)
\end{aligned}
$$




$$
\Rightarrow \quad x(y, z, w)-(y, z, w) x=(x, y, z w)+(x, z, w y)+(x, w, y z)
$$

O lema anterior nos mostra que não são necessários os termos com parêntesis à direita. De fato, fazendo $(x, y, z)=a$ temos:

$$
(x y) a-x(y a)=(x, y, a) \stackrel{(i)}{=}[x, y] a=(x y) a-(y x) a \Rightarrow x(y a)=(y x) a
$$

Analogamente obtemos:

$$
\begin{aligned}
& x(a y)=(a x) y, \quad y(x a)=(x y) a, \quad a(x y)=(x a) y, \\
& y(a x)=(a y) x, \quad a(y x)=(y a) x .
\end{aligned}
$$

Teorema 4.6.11. Se $f$ é uma identidade de grau $[2,2,1]$ pertencente ao ideal associador, então $f=0$.

Demonstração: Podemos escrever:

$$
f=\alpha_{1}(x y) a+\alpha_{2}(y x) a+\alpha_{3}(x a) y+\alpha_{4}(a x) y+\alpha_{5}(y a) x+\alpha_{6}(a y) x
$$

Vamos fazer substituições em $x, y, z$ por elementos da base dos octônios.

Substituição (1): $x=1+e_{1}, y=1+e_{2}, z=e_{4}$

$$
\begin{aligned}
(x y) a & =\left(\left(1+e_{1}\right)\left(1+e_{2}\right)\right)\left(1+e_{1}, 1+e_{2}, e_{4}\right) \\
& =\left(1+e_{1}+e_{2}+e_{3}\right)\left(e_{1}, e_{2}, e_{4}\right)=\left(1+e_{1}+e_{2}+e_{3}\right)\left(2 e_{7}\right) \\
& =2\left(e_{7}+e_{6}-e_{5}-e_{4}\right) \\
(y x) a & =\left(\left(1+e_{2}\right)\left(1+e_{1}\right)\right)\left(1+e_{1}, 1+e_{2}, e_{4}\right) \\
& =\left(1+e_{1}+e_{2}-e_{3}\right)\left(e_{1}, e_{2}, e_{4}\right)=\left(1+e_{1}+e_{2}-e_{3}\right)\left(2 e_{7}\right) \\
& =2\left(e_{7}+e_{6}-e_{5}+e_{4}\right)
\end{aligned}
$$

Omitimos os detalhes dos próximos cálculos, indicando apenas os resultados obtidos, que são:

$$
\begin{aligned}
& (x a) y=2\left(e_{7}+e_{6}+e_{5}+e_{4}\right), \quad(a x) y=2\left(e_{7}-e_{6}+e_{5}-e_{4}\right), \\
& (y a) x=2\left(e_{7}-e_{6}-e_{5}-e_{4}\right), \quad(a y) x=2\left(e_{7}-e_{6}+e_{5}+e_{4}\right)
\end{aligned}
$$

A expressão de $f$ fica então, após cancelarmos o 2 :

$$
\begin{array}{r}
f=\alpha_{1}\left(e_{7}+e_{6}-e_{5}-e_{4}\right)+\alpha_{2}\left(e_{7}+e_{6}-e_{5}+e_{4}\right)+\alpha_{3}\left(e_{7}+e_{6}+e_{5}+e_{4}\right) \\
+\alpha_{4}\left(e_{7}-e_{6}+e_{5}-e_{4}\right)+\alpha_{5}\left(e_{7}-e_{6}-e_{5}-e_{4}\right)+\alpha_{6}\left(e_{7}-e_{6}+e_{5}+e_{4}\right)
\end{array}
$$


Substituição (2): $e_{1}, e_{1}+e_{2}, e_{4}$

$$
\begin{aligned}
(x y) a & =\left(e_{1}\left(e_{1}+e_{2}\right)\right)\left(e_{1}, e_{1}+e_{2}, e_{4}\right) \\
& =\left(-1+e_{3}\right)\left(e_{1}, e_{2}, e_{4}\right)=\left(-1+e_{3}\right)\left(2 e_{7}\right)=2\left(-e_{7}-e_{4}\right) \\
(y x) a & =\left(\left(e_{1}+e_{2}\right) e_{1}\right)\left(e_{1}, e_{1}+e_{2}, e_{4}\right) \\
& =\left(-1-e_{3}\right)\left(e_{1}, e_{2}, e_{4}\right)=\left(-1-e_{3}\right)\left(2 e_{7}\right)=2\left(-e_{7}+e_{4}\right)
\end{aligned}
$$

Após os devidos cálculos obtemos os demais elementos:

$$
\begin{aligned}
& (x a) y=2\left(e_{7}+e_{4}\right), \quad(a x) y=2\left(-e_{7}-e_{4}\right), \quad(y a) x=2\left(e_{7}-e_{4}\right), \\
& \left(\text { ay } x=2\left(-e_{7}+e_{4}\right)\right.
\end{aligned}
$$

Resulta, para $f$ :

$$
\begin{aligned}
f & =\alpha_{1}\left(-e_{7}-e_{4}\right)+\alpha_{2}\left(-e_{7}+e_{4}\right)+\alpha_{3}\left(e_{7}+e_{4}\right)+\alpha_{4}\left(-e_{7}-e_{4}\right) \\
& +\alpha_{5}\left(e_{7}-e_{4}\right)+\alpha_{6}\left(-e_{7}+e_{4}\right)
\end{aligned}
$$

Igualando a 0 as expressões (1) e (2), pois $f$ é uma identidade, obtemos, após colocar em evidência os $e_{i}$ :

$$
\begin{aligned}
\left(\alpha_{1}+\alpha_{2}+\alpha_{3}+\alpha_{4}+\alpha_{5}+\alpha_{6}\right) e_{7} & =0 \\
\left(\alpha_{1}+\alpha_{2}+\alpha_{3}-\alpha_{4}-\alpha_{5}-\alpha_{6}\right) e_{6} & =0 \\
\left(-\alpha_{1}-\alpha_{2}+\alpha_{3}+\alpha_{4}-\alpha_{5}+\alpha_{6}\right) e_{5} & =0 \\
\left(-\alpha_{1}+\alpha_{2}+\alpha_{3}-\alpha_{4}-\alpha_{5}+\alpha_{6}\right) e_{4} & =0 \\
\left(-\alpha_{1}-\alpha_{2}+\alpha_{3}-\alpha_{4}+\alpha_{5}-\alpha_{6}\right) e_{7} & =0 \\
\left(-\alpha_{1}+\alpha_{2}+\alpha_{3}-\alpha_{4}-\alpha_{5}+\alpha_{6}\right) e_{4} & =0
\end{aligned}
$$

Os coeficientes dos $e_{i}$ devem se anular, o que resulta em um sistema homogêneo nas incógnitas $\alpha_{1}, \ldots, \alpha_{6}$. Após escalonamento chegamos a:

$$
\begin{aligned}
\alpha_{1}+\alpha_{2}+\alpha_{3}+\alpha_{4}+\alpha_{5}+\alpha_{6} & =0 \\
\alpha_{2}+\alpha_{3}+\alpha_{6} & =0 \\
\alpha_{3}+\alpha_{4}+\alpha_{6} & =0 \\
\alpha_{4}-\alpha_{5}+\alpha_{6} & =0 \\
-\alpha_{5} & =0
\end{aligned}
$$

Trata-se de um sistema indeterminado com variável livre $\alpha_{6}$. Sua solução é: 


$$
\alpha_{3}=\alpha_{5}=0, \quad \alpha_{4}=\alpha_{2}=-\alpha_{6}, \quad \alpha_{1}=\alpha_{6} .
$$

Assim, $f=\alpha_{6}((x y) a-(y x) a-(a x) y+(a y) x)=\alpha_{6} g$.

$$
\begin{aligned}
g & =(x y) a-(y x) a-(a x) y+(a y) x \\
& =[x, y] a-(a, x, y)-a(x y)+(a, y, x)+a(y x) \\
& =[x, y] a-2(a, x, y)-a[x, y]
\end{aligned}
$$

Pelo Lema 4.6.10 (iii): $[x, y] \circ a=0 \Rightarrow[x, y] a=-a[x, y]$

e por 4.6.10 (i): $\quad(x, y, a)=[x, y] a$

o que nos leva a: $\quad g=2(x, y, a)-2(x, y, a)=0$.

E portanto: $\quad f=0$.

\section{Identidades de grau $[2,1,1,1]$}

Seja $f(x, y, z, w)$ uma identidade de grau $[2,1,1,1]$. Então $\phi f$ está no ideal das identidades associativas. Vamos explicitar os elementos geradores deste ideal. Veremos que todos são conseqüência de $\chi$ e de $\kappa$. Por fim, se uma identidade $f$ pertence ao ideal associador de grau $[2,1,1,1]$, provaremos que $f=0$.

Proposição 4.6.12. No ideal das identidades associativas, o subespaço das identidades de grau $[2,1,1,1]$ é gerado pelos seguintes elementos:

$$
\begin{array}{llll}
x \circ s_{4}(x, y, z, w), & {\left[x, s_{4}(x, y, z, w)\right],} & s_{4}\left(x^{2}, y, z, w\right), & s_{4}(x, x \circ y, z, w) \\
s_{4}(x,[x, y], z, w), & {[x,[x, y] \circ[z, w]],} & {[y,[x, z] \circ[x, w]]}
\end{array}
$$

mais as permutações cíclicas de $y, z, w$ nos 4 últimos polinômios, a saber:

$$
\begin{array}{llll}
s_{4}(x, x \circ z, w, y), & s_{4}(x, x \circ w, y, z), & s_{4}(x,[x, z], w, y), & s_{4}(x,[x, w], y, z) \\
{[x,[x, z] \circ[w, y]],} & {[x,[x, w] \circ[y, z]],} & {[z,[x, w] \circ[x, y]],} & {[w,[x, y] \circ[x, z]] .}
\end{array}
$$

Demonstração: Uma identidade de grau $[2,1,1,1]$ é formada por monômios de grau 2 em $x$ e grau 1 em $y, z, w$. Já vimos que as identidades de grau 5 em Ass $[X]$ (ou em $\left.M_{2}(F)\right)$ são de um dos seguintes tipos:

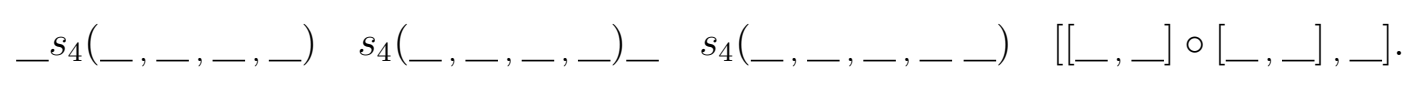


Obtemos os geradores do subespaço das identidades de grau $[2,1,1,1]$, preenchendo os argumentos acima pelas variáveis $x, y, z, w$ com grau $[2,1,1,1]$, de modo a termos todas as possibilidades de distribuições dessas variáveis. Um simples raciocínio combinatório permite concluir que este preenchimento produz os mesmos elementos indicados pela proposição.

Proposição 4.6.13. No conjunto de geradores do subespaço das identidades de grau $[2,1,1,1]$, tem-se:

(i) $x \circ s_{4}(x, y, z, w)$ é conseqüência de $\kappa$ e de $s_{4}\left(x^{2}, y, z, w\right)$;

(ii) $\left[x, s_{4}(x, y, z, w)\right]$ é conseqüência de $\chi$;

(iii) $s_{4}(x,[x, y], z, w)$ é conseqüência de $\chi$;

(iv) $s_{4}(x, x \circ y, z, w)$ é conseqüência de $\kappa$ e de $s_{4}\left(x^{2}, y, z, w\right)$;

(v) $[x,[x, y] \circ[z, w]]$ e $[y,[x, z] \circ[x, w]]$ são conseqüências de $\chi$.

\section{Demonstração:}

(i) Pela Proposição 4.6.4: $\kappa(x ; y, z, w)=s_{4}\left(y, z, w, x^{2}\right)-s_{4}(y, z, w, x) \circ x$ Mas, notando que: $s_{4}\left(y, z, w, x^{2}\right)=s_{4}\left(x^{2}, y, z, w\right)$, $s_{4}(y, z, w, x) \circ x=x \circ s_{4}(y, z, w, x)$ e $s_{4}(y, z, w, x)=s_{4}(x, y, z, w)$, podemos escrever: $x \circ s_{4}(x, y, z, w)=s_{4}\left(x^{2}, y, z, w\right)-\kappa(x ; y, z, w)$

(ii) $\left[x, s_{4}(x, y, z, w)\right]=x([x, y] \circ[z, w]+[x, z] \circ[w, y]+[x, w] \circ[y, z])$

$$
\begin{aligned}
& -([x, y] \circ[z, w]+[x, z] \circ[w, y]+[x, w] \circ[y, z]) x \\
& =\underbrace{[x,[x, y] \circ[z, w]]}_{(1)}+\underbrace{[x,[x, z] \circ[w, y]]}_{(2)}+\underbrace{[x,[x, w] \circ[y, z]]}_{(3)}
\end{aligned}
$$

Vamos linearizar $\chi=\left[[x, y]^{2}, x\right]$ para obter as parcelas (1), (2) e (3).

$\left[[x+z+w, y+x+z]^{2}, x+z+w\right]$

$=\left[([x, y]+[x, z]+[z, y]+[w, y]+[w, x]+[w, z])^{2}\right.$

$+[x, y] \circ[x, z]+[x, y] \circ[z, y]+[x, y] \circ[w, y]+[x, y] \circ[w, x]+\underbrace{[x, y] \circ[w, z]}_{(1)}$ 
$+[x, z] \circ[z, y]+\underbrace{[x, z] \circ[w, y]}_{(2)}+[x, z] \circ[w, x]+[x, z] \circ[w, z]$

$+[z, y] \circ[w, y]+\underbrace{[z, y] \circ[w, x]}_{(3)}+[z, y] \circ[w, z]$

$+[w, y] \circ[w, x]+[w, y] \circ[w, z]$

$+[w, x] \circ[w, z], x+z+w]$

Estão assinalados os termos termos (1), (2) e (3), que formarão com $x$ os comutadores procurados:

$[[x, y] \circ[w, z], x]=[x,[x, y] \circ[z, w]]$

$[[x, z] \circ[w, y], x]=-[x,[x, z] \circ[w, y]]$

$[[z, y] \circ[w, x], x]=-[x,[x, w] \circ[y, z]]$

(iii) Pelo Lema 4.6.5 (i), temos:

$s_{4}(x,[x, y], z, w)=[x,[x, y]] \circ[z, w]+[x, z] \circ[w,[x, y]]+[x, w] \circ[[x, y], z]$

Fazendo em 4.6 .5 (ii), $y=[x, y]$ e $z=[z, w]$ e aplicando na primeira parcela de (1):

$[x,[x, y]] \circ[z, w]=\underbrace{[x,[x, y] \circ[z, w]]}_{(a)}-[x, y] \circ[x,[z, w]]$

Fazendo em 4.6 .5 (ii), $x=w, y=[x, y]$ e $z=[x, z]$ e aplicando na segunda parcela de (1):

$[x, z] \circ[w,[x, y]]=[w,[x, y]] \circ[x, z]=\underbrace{[w,[x, y] \circ[x, z]]}_{(b)}-[x, y] \circ[w,[x, z]]$

Fazendo em 4.6 .5 (ii), $x=z, y=[x, y]$ e $z=[x, w]$ e aplicando na terceira parcela de (1):

$[x, w] \circ[[x, y], z]=-[z,[x, y]] \circ[x, w]=\underbrace{-[z,[x, y] \circ[x, w]]}_{(c)}+[x, y] \circ[z,[x, w]]$

Levando (2), (3) e (4) em (1) e agrupando as expressões (a), (b) e (c), nos dá:

$$
\begin{aligned}
s_{4}(x,[x, y], z, w) & =[x,[x, y] \circ[z, w]]+[w,[x, y] \circ[x, z]]-[z,[x, y] \circ[x, w]] \\
& +[x, y] \circ([x,[w, z]]+[w,[z, x]]+[z,[x, w]])
\end{aligned}
$$

Notando que, por 4.6.5 (iii) (identidade de Jacobi), 
$[x,[w, z]]+[w,[z, x]]+[z,[x, w]]=0$, podemos escrever:

$s_{4}(x,[x, y], z, w)=[x,[x, y] \circ[z, w]]+[w,[x, y] \circ[x, z]]-[z,[x, y] \circ[x, w]]$

Portanto, $s_{4}$ é conseqüência de $\chi$.

(iv) Fazendo $y=x$ na Proposição 4.6.4, temos: $\kappa(x ; x, z, w)=s_{4}\left(x, z, w, x^{2}\right)$.

Linearizando com $x+\lambda y$ :

$$
\begin{aligned}
\kappa(x+\lambda y ; & x+\lambda y, z, w)=s_{4}\left(x+\lambda y,(x+\lambda y)^{2}, z, w\right) \\
& =s_{4}\left(x, x^{2}, z, w\right)+\lambda s_{4}(x, x \circ y, z, w)+\lambda^{2} s_{4}\left(x, y^{2}, z, w\right) \\
& +\lambda s_{4}\left(y, x^{2}, z, w\right)+\lambda^{2}(y, x \circ y, z, w)+\lambda^{3} s_{4}\left(y, y^{2}, z, w\right) \\
& =\kappa(x ; x, z, w)+\lambda\left(s_{4}(x, x \circ y, z, w)-s_{4}\left(x^{2}, y, z, w\right)\right) \\
& +\lambda^{2}\left(s_{4}(y, x \circ y, z, w)-s_{4}\left(y^{2}, x, z, w\right)\right)+\lambda^{3} \kappa(y ; y, z, w)
\end{aligned}
$$

Fazendo: $K=\kappa(x+\lambda y ; x+\lambda y, z, w), \quad K_{1}=\kappa(x ; x, z, w)$,

$$
\begin{aligned}
& K_{2}=\kappa(y ; y, z, w), \quad L=s_{4}(x, x \circ y, z, w)-s_{4}\left(x^{2}, y, z, w\right) \\
& T=s_{4}(y, x \circ y, z, w)-s_{4}\left(y^{2}, x, z, w\right),
\end{aligned}
$$

escrevemos: $K=K_{1}+\lambda L+\lambda^{2} T+\lambda^{3} K_{2}$

Substituindo alguns valores para $\lambda$ :

$\lambda=1: \quad K=K_{1}+L+T+K_{2} ; \quad \lambda=2: \quad K^{\prime}=K_{1}+2 L+4 T+8 K_{2}$

$\Rightarrow \quad L=\frac{1}{2}\left(4\left(K_{2}+K\right)-K^{\prime}-3 K_{1}\right)$

$\mathrm{Ou}$, mais explicitamente:

$$
\begin{aligned}
\lambda=1: \quad \kappa(x+y ; x+y, z, w)= & \kappa(x ; x, z, w) \\
+ & {\left[s_{4}(x, x \circ y, z, w)-s_{4}\left(x^{2}, y, z, w\right)\right] } \\
+ & {\left[s_{4}(y, x \circ y, z, w)-s_{4}\left(y^{2}, x, z, w\right)\right] } \\
+ & \kappa(y ; y, z, w) \\
\lambda=2: \quad \kappa(x+2 y ; x+2 y, z, w) & =\kappa(x ; x, z, w) \\
& +2\left[s_{4}(x, x \circ y, z, w)-s_{4}\left(x^{2}, y, z, w\right)\right] \\
& +4\left[s_{4}(y, x \circ y, z, w)-s_{4}\left(y^{2}, x, z, w\right)\right] \\
& +8 \kappa(y ; y, z, w)
\end{aligned}
$$




$$
\begin{aligned}
& \kappa(x+2 y ;+2 y, z, w)-4 \kappa(x+y ; x+y, z, w) \\
&=-3 \kappa(x ; x, z, w)-2\left(s_{4}(x, x \circ y, z, w)-s_{4}\left(x^{2}, y, z, w\right)\right) \\
&+4 \kappa(y ; y, z, w) \\
& \Rightarrow \quad s_{4}(x ; x \circ y, z, w)-s_{4}\left(x^{2}, y, z, w\right) \\
&=-\frac{1}{2}[\kappa(x+2 y ; x+2 y, z, w)-4(\kappa(x+y ; x+y, z, w)+\kappa(y ; y, z, w)) \\
&+3 \kappa(y ; y ; z ; w)]
\end{aligned}
$$

(v) É imediato.

Lembrando que estamos considerando uma identidade $f \in A l t[X]$ de grau $[2,1,1,1]$, com as três últimas variáveis alternadas. Conforme a proposição anterior sua imagem $\phi f$ em $A s s[X]$ é conseqüência de $\chi$, de $\kappa$ e de $s_{4}\left(x^{2}, y, z, w\right)$. Podemos escrever então:

$$
\phi f=\lambda \kappa+\mu \chi+\gamma s_{4}
$$

onde $\lambda, \mu, \gamma \in F$.

Notando que $\phi \kappa=\kappa, \phi \chi=\chi \quad$ e $\quad \phi s_{4}^{i}=s_{4}, \forall i=1, \ldots, 5$, podemos fazer:

$$
f^{\prime}=f-\lambda \kappa-\mu \chi-\sum_{i} \gamma_{i} s_{4}^{i} \Rightarrow \phi f^{\prime}=\phi f-\lambda \kappa-\mu \chi-\gamma s_{4}=\phi f-\phi f=0 .
$$

Obtivemos assim, uma identidade $f^{\prime}$ no ideal associador. Mostraremos que $f^{\prime}=0$, mas antes iremos explicitar os elementos do ideal associador das identidades de grau $[2,1,1,1]$. Os elementos geradores deste ideal são: $(x a) y,(a x) y,(x y) a,(y x) a,(a y) x$ e $(y a) x$, onde $a=(x, z, w)$, mais as permutações cíclicas de $y, z, w$ e $x^{2} b, x b x, b x^{2}$, onde $b=(y, z, w)$. Deveríamos ter também os termos com parêntesis à direita, $x(a y), a(x y), x(y a), y(x a), a(y x), y(a x)$. O lema a seguir mostra que bastam os nove primeiros termos e mais apenas um com parêntesis à direita.

Lema 4.6.14. O ideal associador das identidades de grau $[2,1,1,1]$ alternadas nas variáveis lineares, é gerado pelos seguintes elementos:

$$
\begin{aligned}
& (x y)(x, z, w)+(x z)(x, w, y)+(x w)(x, y, z), \\
& (y x)(x, z, w)+(z x)(x, w, y)+(w x)(x, y, z),
\end{aligned}
$$




$$
\begin{gathered}
(x(x, z, w)) y+(x(x, w, y)) z+(x(x, y, z)) w, \\
(y(x, z, w)) x+(z(x, w, y)) x+(w(x, y, z)) x, \\
((x, z, w) x) y+((x, w, y) x) z+((x, y, z) x) w, \\
((x, z, w) y) x+((x, w, y) z) x+((x, y, z) w) x, \\
x(y(x, z, w))+x(z(x, w, y))+x(w(x, y, z)), \\
x^{2}(y, z, w), \quad x(y, z, w) x, \quad(y, z, w) x^{2} .
\end{gathered}
$$

Demonstração: Basta mostrar que apenas um termo com parêntesis à direita é necessário. Consideremos os produtos à direita: $a(b c), b(a c), a(c b), c(a b), b(c a), c(b a)$.

Fixando um desses produtos, digamos $a(b c)$, vamos tentar obter os outros. Usaremos a alternatividade do associador.

$$
\begin{aligned}
(b, a, c) & =(b a) c-b(a c)=-(a, b, c)=a(b c)-(a b) c \\
& \Rightarrow b(a c)=(b a) c+(a b) c-a(b c) \\
(c, a, b) & =(c a) b-c(a b)=(a, b, c)=(a b) c-a(b c) \\
& \Rightarrow c(a b)=(c a) b-(a b) c+a(b c)
\end{aligned}
$$

Para os demais produtos os cálculos são inteiramente análogos.

Definição 4.6.2. Chama-se triplo produto de Jordan à expressão:

$$
\{x y z\}=(x y) z+z(y x)
$$

Teorema 4.6.15. Se $f$ é uma identidade no ideal associador de grau $[2,1,1,1]$, então $f=0$.

Demonstração: Vamos fazer substituições nos elementos geradores, por elementos da base dos octônios.

(1) $x=1+e_{1}, \quad y=1+e_{2}, \quad z=e_{4}, \quad w=e_{3}$

$$
\begin{aligned}
& (x, z, w)=\left(1+e_{1}, e_{4}, e_{3}\right)=\left(e_{1}, e_{4}, e_{3}\right)=\left(e_{1} e_{4}\right) e_{3}-e_{1}\left(e_{4} e_{3}\right)=2 e_{6} \\
& (x, w, y)=\left(e_{1}, e_{3}, e_{2}\right)=\left(e_{1} e_{3}\right) e_{2}-e_{1}\left(e_{3} e_{2}\right)=0 \\
& (x, y, z)=\left(e_{1}, e_{2}, e_{4}\right)=2 e_{7}
\end{aligned}
$$


$(y, z, w)=\left(e_{2}, e_{4}, e_{3}\right)=-2 e_{5}$

Agora calculamos cada um dos dez termos correspondentes aos elementos do conjunto gerador de $I$. Chamaremos estes termos de $u_{1}, \ldots, u_{10}$.

$$
\begin{aligned}
& (x y)(x, z, w)=\left(\left(1+e_{1}\right)\left(1+e_{2}\right)\right)\left(2 e_{6}\right)=2\left(e_{6}-e_{7}-e_{4}+e_{5}\right) \\
& (x w)(x, y, z)=\left(\left(1+e_{1}\right) e_{3}\right) 2 e_{7}=-2\left(e_{4}-e_{5}\right) \\
& \Rightarrow u_{1}=-4 e_{4}+4 e_{5}+2 e_{6}-2 e_{7} \\
& (y x)(x, z, w)=\left(\left(1+e_{2}\right)\left(1+e_{1}\right)\right)\left(2 e_{6}\right)=2\left(e_{6}-e_{7}-e_{4}-e_{5}\right) \\
& (w x)(x, y, z)=\left(e_{3}\left(1+e_{1}\right)\right)\left(2 e_{7}\right)=-2\left(e_{4}+e_{5}\right) \\
& \Rightarrow u_{2}=4 e_{4}-4 e_{5}+2 e_{6}-2 e_{7}
\end{aligned}
$$

Prosseguindo com esses cálculos, que indicaremos de forma sucinta, obtemos os demais valores:

$$
\begin{aligned}
& (x(x, z, w)) y=2\left(e_{4}-e_{5}+e_{6}-e_{7}\right), \quad(x(x, z, y)) w=-2\left(-e_{4}+e_{5}\right) \\
& \Rightarrow \quad u_{3}=4 e_{4}-4 e_{5}+2 e_{6}-2 e_{7} \\
& (y(x, z, w)) x=2\left(-e_{4}+e_{5}+e_{6}+e_{7}\right), \quad(w(x, y, z)) x=-2\left(e_{4}-e_{5}\right) \\
& \Rightarrow \quad u_{4}=-4 e_{4}+2 e_{6}+2 e_{7}+4 e_{5} \\
& ((x, z, w) x) y=2\left(e_{4}+e_{5}+e_{6}+e_{7}\right), \quad((x, y, z) x) w=-2\left(-e_{4}-e_{5}\right) \\
& \Rightarrow \quad u_{5}=4 e_{4}-4 e_{5}+2 e_{6}+2 e_{7} \\
& ((x, z, w) y) x=2\left(e_{6}+e_{4}+e_{7}-e_{5}\right), \quad((x, y, z) w) x=2\left(e_{4}-e_{5}\right) \\
& \Rightarrow \quad u_{6}=-4 e_{4}-4 e_{5}+2 e_{6}+2 e_{7} \\
& x(y(x, z, w))=2\left(-e_{4}-e_{5}+e_{6}-e_{7}\right), \quad x(w(x, y, z))=-2\left(e_{4}+e_{5}\right) \\
& \Rightarrow \quad u_{7}=-4 e_{4}-4 e_{5}+2 e_{6}-2 e_{7} \\
& x^{2}(y, z, w)=4 e_{4} \quad \Rightarrow \quad u_{8}=4 e_{4} ; \quad x(y, z, w) x=-4 e_{5} \quad \Rightarrow \quad u_{9}=-4 e_{5} \\
& (y, z, w) x^{2}=-4 e_{4} \quad \Rightarrow \quad u_{10}=-4 e_{4}
\end{aligned}
$$

A identidade $f$ pode ser escrita: $f=\alpha_{1} u_{1}+\cdots+\alpha_{10} u_{10}$

$$
\Rightarrow f=\left(-4 e_{4}+4 e_{5}+2 e_{6}-2 e_{7}\right) \alpha_{1}+\cdots+\left(-4 e_{4}\right) \alpha_{10}
$$


Colocando em evidência os $e_{i}$ obtemos um conjunto de 4 equações nas variáveis $\alpha_{i}$ :

$$
\begin{array}{rrr}
-\alpha_{1}-\alpha_{2}+\alpha_{3}-\alpha_{4}+\alpha_{5}+\alpha_{6}-\alpha_{7}+\alpha_{8} & -\alpha_{10}=0 \\
\alpha_{1}+\alpha_{2}+\alpha_{3}+\alpha_{4}+\alpha_{5}+\alpha_{6}+\alpha_{7} & =0 \\
\alpha_{1}+\alpha_{2}+\alpha_{3}-\alpha_{4}-\alpha_{5}-\alpha_{6}+\alpha_{7} & =0 \\
\alpha_{1}-\alpha_{2}-\alpha_{3}+\alpha_{4}+\alpha_{5}-\alpha_{6}-\alpha_{7} & -\alpha_{9} & =0
\end{array}
$$

Com estas equações podemos formar as 4 primeiras linhas de uma matriz:

$\begin{array}{lrrrrrrrrrr}\text { L1: } & -1 & -1 & 1 & -1 & 1 & 1 & -1 & 1 & 0 & -1 \\ \text { L2: } & 1 & 1 & 1 & 1 & 1 & 1 & 1 & 0 & 0 & 0 \\ \text { L3: } & 1 & 1 & 1 & -1 & -1 & -1 & 1 & 0 & 0 & 0 \\ \text { L4: } & 1 & -1 & -1 & 1 & 1 & -1 & -1 & 0 & -1 & 0\end{array}$

(2) $x=1+e_{1}, \quad y=e_{2}, \quad z=e_{4}, \quad w=e_{6}$

$(x, z, w)=\left(e_{1}, e_{4}, e_{6}\right)=-2 e_{3} ; \quad(x, w, y)=\left(e_{1}, e_{6}, e_{2}\right)=-2 e_{5}$

$(x, y, z)=\left(e_{1}, e_{2}, e_{4}\right)=2 e_{7} ; \quad(y, z, w)=\left(e_{2}, e_{4}, e_{6}\right)=0$

$(x y)(x, z, w)=-2 e_{1}+2, \quad(x z)(x, w, y)=-2 e_{1}+2, \quad(x w)(x, y, z)=-2 e_{1}+2$

$\Rightarrow u_{1}=-6 e_{1}+6$

Efetuando os cálculos correspondentes obtemos para os outros $u_{i}$ :

$u_{2}=-6 e_{1}-6, \quad u_{3}=6 e_{1}-6, \quad u_{4}=-6 e_{1}+6, \quad u_{5}=6 e_{1}+6$,

$u_{6}=6 e_{1}-6, \quad u_{7}=-6 e_{1}+6, \quad u_{8}=u_{9}=u_{10}=0$

Mais duas equações são obtidas, que resultarão nas linhas 5 e 6 da matriz $10 \times 10$ :

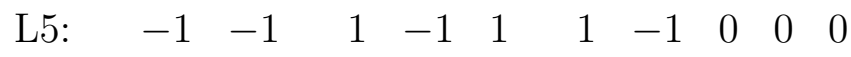

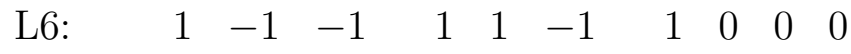

(3) $x=1+e_{1}, \quad y=e_{2}, \quad z=e_{4}, \quad w=-e_{7}$

$(x, z, w)=\left(e_{1}, e_{4},-e_{7}\right)=-2 e_{2} ; \quad(x, w, y)=\left(e_{1},-e_{7}, e_{2}\right)=-2 e_{4}$

$(x, y, z)=\left(e_{1}, e_{2}, e_{4}\right)=2 e_{7} ; \quad(y, z, w)=\left(e_{2}, e_{4}, e_{6}\right)=2 e_{1}$

Indicamos a seguir os valores dos $u_{i}$ resultantes desta substituição:

$u_{1}=6 e_{1}+6, \quad u_{2}=-6 e_{1}+6, \quad u_{3}=6 e_{1}+6, \quad u_{4}=6 e_{1}+6$, 
$u_{5}=-6 e_{1}+6, \quad u_{6}=6 e_{1}+6, \quad u_{7}=6 e_{1}+6, \quad u_{8}=u_{9}=u_{10}=-4$

Obtemos mais duas equações que nos fornecem as linhas 7 e 8 da matriz:

L7: $\quad \begin{array}{lllllllllll}3 & 3 & 3 & 3 & 3 & 3 & 3 & -2 & -2 & -2\end{array}$

L8: $\quad \begin{array}{rrrrrrrrrr}1 & -1 & 1 & 1 & -1 & 1 & 1 & 0 & 0 & 0\end{array}$

(4) $x=e_{1}, \quad y=e_{2}, \quad z=e_{4}, \quad w=e_{3}$

$(x, z, w)=\left(e_{1}, e_{4}, e_{3}\right)=2 e_{6} ; \quad(x, w, y)=\left(e_{1}, e_{3}, e_{2}\right)=0$

$(x, y, z)=\left(e_{1}, e_{2}, e_{4}\right)=2 e_{7} ; \quad(y, z, w)=\left(e_{2}, e_{4}, e_{3}\right)=-2 e_{5}$

Valores dos $u_{i}$ resultantes desta substituição:

$u_{1}=4 e_{7}, \quad u_{2}=-4 e_{7}, \quad u_{3}=-4 e_{7}, \quad u_{4}=4 e_{7}, \quad u_{5}=4 e_{7}$,

$u_{6}=-4 e_{7}, \quad u_{7}=-4 e_{7}, \quad u_{8}=2 e_{7}, \quad u_{9}=-2 e_{7}, \quad u_{10}=2 e_{7}$

A equação obtida nos dará a linha 9 da matriz:

L9: $\quad \begin{array}{llllllllll}2 & -2 & -2 & 2 & 2 & -2 & -2 & 1 & -1 & 1\end{array}$

(5) $x=e_{1}, \quad y=e_{1}, \quad z=e_{2}, \quad w=e_{4}$

$(x, z, w)=\left(e_{1}, e_{2}, e_{4}\right)=2 e_{7} ; \quad(x, w, y)=\left(e_{1}, e_{4}, e_{1}\right)=0$

$(x, y, z)=\left(e_{1}, e_{1}, e_{2}\right)=0 ; \quad(y, z, w)=\left(e_{1}, e_{2}, e_{4}\right)=2 e_{7}$

Valores dos $u_{i}$ resultantes desta substituição:

$u_{1}=2 e_{6}, \quad u_{2}=2 e_{6}, \quad u_{3}=-2 e_{6}, \quad u_{4}=-2 e_{6}, \quad u_{5}=2 e_{6}$,

$u_{6}=2 e_{6}, \quad u_{7}=2 e_{6}, \quad u_{8}=2 e_{6}, \quad u_{9}=-2 e_{6}, \quad u_{10}=2 e_{6}$

A equação obtida nos dará a linha 10 da matriz:

\section{L10: $\quad \begin{array}{lllllllllll} & 1 & 1 & -1 & -1 & 1 & 1 & 1 & 1 & -1 & 1\end{array}$}

A matriz dos coeficientes do sistema homogêneo nas incógnitas $\alpha_{1}, \ldots, \alpha_{10}$ obtida por meio das substituições indicadas acima, é a seguinte: 


$$
\left(\begin{array}{rrrrrrrrrr}
-1 & -1 & 1 & -1 & 1 & 1 & -1 & 1 & 0 & -1 \\
1 & 1 & 1 & 1 & 1 & 1 & 1 & 0 & 0 & 0 \\
1 & 1 & 1 & -1 & -1 & -1 & 1 & 0 & 0 & 0 \\
1 & -1 & -1 & 1 & 1 & -1 & -1 & 0 & -1 & 0 \\
-1 & -1 & 1 & -1 & 1 & 1 & -1 & 0 & 0 & 0 \\
1 & -1 & -1 & 1 & 1 & -1 & 1 & 0 & 0 & 0 \\
3 & 3 & 3 & 3 & 3 & 3 & 3 & -2 & -2 & -2 \\
1 & -1 & 1 & 1 & -1 & 1 & 1 & 0 & 0 & 0 \\
2 & -2 & -2 & 2 & 2 & -2 & -2 & 1 & -1 & 1 \\
1 & 1 & -1 & -1 & 1 & 1 & 1 & 1 & -1 & 1
\end{array}\right)
$$

Sua forma escalonada é dada a seguir:

$$
\left(\begin{array}{rrrrrrrrrr}
1 & 0 & 0 & 0 & 0 & 0 & 0 & 0 & 0 & 2 \\
0 & 1 & 0 & 0 & & & & & & 0 \\
& 0 & 1 & 0 & & & & & & -1 \\
& & 0 & 1 & 0 & & & & & -1 \\
& & & 0 & 1 & 0 & & & & 0 \\
& & & & 0 & 1 & 0 & & & 1 \\
& & & & & 0 & 1 & 0 & & -1 \\
& & & & & & 0 & 1 & 0 & -1 \\
& & & & & & 0 & 0 & 1 & 2 \\
& & & & & & 0 & 0 & 0
\end{array}\right)
$$

Resultam as seguintes relações entre as variáveis, onde fizemos a variável livre $\alpha_{10}=\alpha$ :

$\alpha_{1}=\alpha_{9}=-2 \alpha ; \quad \alpha_{2}=\alpha_{5}=0 ; \quad \alpha_{3}=\alpha_{4}=\alpha_{7}=\alpha_{8}=\alpha ; \quad \alpha_{6}=-\alpha$.

Substituindo estes valores na expressão de $f$, teremos:

$$
\begin{aligned}
f(x, y, z, w)= & \alpha(-2((x y)(x, z, w)+(x z)(x, w, y)+(x w)(x, y, z)) \\
& +((x(x, z, w)) y+(x(x, w, y)) z+(x(x, y, z)) w) \\
& +((y(x, z, w)) x+(z(x, w, y)) x+(w(x, y, z)) x) \\
& -(((x, z, w) y) x+((x, w, y) z) x+((x, y, z) w) x) \\
& +(x(y(x, z, w))+x(z(x, w, y))+x(w(x, y, z))) \\
& \left.+x^{2}(y, z, w)-2 x(y, z, w) x+(y, z, w) x^{2}\right)
\end{aligned}
$$

Provaremos que este polinômio é igual a zero em $A l t[X]$.

\section{Afirmação:}

$f=[x,[x,(y, z, w)]]+\sum_{(y, z, w)}(\{x(x, z, w) y\}-\{x y(x, z, w)\}-(x, y,(x, z, w)))$ 
onde $\sum_{(y, z, w)}$ indica a soma sobre todas as permutações cíclicas de $y, z, w$. Analisemos cada parcela.

$$
\begin{aligned}
{[x,[x,(y, z, w)]] } & =[x, x(y, z, w)-(y, z, w) x]=[x, x(y, z, w)]-[x,(y, z, w) x] \\
& =x(x(y, z, w))-x(y, z, w) x-x((y, z, w) x)+((y, z, w) x) x \\
& =x^{2}(y, z, w)-2 x(y, z, w)+(y, z, w) x^{2}
\end{aligned}
$$

Reconhecemos aí os 3 últimos termos da expressão de $f$.

$\{x(x, z, w) y\}=(x(x, z, w)) y+(y(x, z, w)) x$. Fazendo as permutações cíclicas em $y, z, w$, temos:

$$
\begin{aligned}
\sum_{(y, z, w)}\{x(x, z, w) y\} & =(x(x, z, w)) y+(y(x, z, w)) x+(x(x, y, z)) w+(w(x, y, z)) x \\
& +(x(x, w, y)) z+(z(x, w, y)) x
\end{aligned}
$$

Aí estão a segunda e a terceira parcelas de $f$.

$\{x y(x, z, w)\}=(x y)(x, z, w)+((x, z, w) y) x$. É fácil ver que as permutações cíclicas em $y, z, w$ do termo acima resultarão na soma da primeira e da quarta parcelas de $f$.

$(x, y,(x, z, w))=(x y)(x, z, w)-x(y(x, z, w))$. Aplicando as permutações cíclicas obtemos a soma da primeira com a quinta parcela de $f$. Verificamos assim que a nova expressão de $f$ corresponde à anterior, o que comprova a afirmação feita.

Vamos agora desenvolver explicitamente a soma cíclica, designando-a por $\sum_{(y, z, w)}$. Teremos então:

$$
\begin{aligned}
\sum_{(y, z, w)} & =\{x(x, z, w) y\}-\{x y(x, z, w)\}-(x, y,(x, z, w)) \\
& +\{x(x, w, y) z\}-\{x z(x, w, y)\}-(x, z,(x, w, y)) \\
& +\{x(x, y, z) w\}-\{z w(x, y, z)\}-(x, w,(x, y, z)) \\
& =\{x(x, z, w) y\}+\{x w(x, z, y)\} \\
& +\{x(x, w, y) z\}+\{x y(x, w, z)\} \\
& +\{x(x, y, z) w\}+\{x z(x, y, w)\} \\
& -(x, y,(x, z, w))-(x, z,(x, w, y))-(x, w,(x, y, z))
\end{aligned}
$$


As parcelas agrupadas duas a duas têm associadores na forma $(x, z, \ldots),(x, w, \ldots)$ e $\left(x, y, \_\right)$respectivamente, o que sugere uma derivação.

Notando que: $(x, z,\{x w y\})=\{(x, z, x) w y\}+\{x(x, z, w) y\}+\{x w(x, z, y)\}$,

$$
\begin{aligned}
& (x, w,\{x y z\})=\{(x, w, x) y z\}+\{x(x, w, y) z\}+\{x y(x, w, z)\} \mathrm{e} \\
& (x, y,\{x z w\})=\{(x, y, x) z w\}+\{x(x, y, z) w\}+\{x z(x, y, w)\}
\end{aligned}
$$

e ainda que $(x, w, x)=(x, y, x)=(x, z, x)=0$, teremos:

$$
\begin{aligned}
\sum_{(y, z, w)} & =((x, z,\{x w y\})+(x, w,\{x y z\})+(x, y,\{x z w\}) \\
& -(x, z,(x, w, y))-(x, w,(x, y, z))-(x, y,(x, z, w))
\end{aligned}
$$

Temos ainda: $\{x w y\}-(x, w, y)=(x w) y+(y w) x-(x w) y+x(w y)=(y w) x+x(w y)$. Daí:

$$
\begin{aligned}
\sum_{(y, z, w)} & =((x, z, x(w y)+(y w) x)+(x, w, x(y z)+(z y) x)+(x, y, x(z w)+(w z) x)) \\
& =((x, z, x(w y))+(x, z,(y w) x)+(x, w, x(y z))+(x, w,(z y) x) \\
& +(x, y, x(z w))+(x, y,(w z) x))
\end{aligned}
$$

Pelo Lema 4.5.3 (i), (ii):

$$
\begin{aligned}
(x, z, x(w y))+(x, z,(y w) x) & =-(x, x(w y), z)-(x,(y w) x, z) \\
& =-(x, w y, z) x-x(x, y w, z) \\
(x, w, x(y z))+(x, w,(z y) x) & =-(x, y z, w) x-x(x, z y, w) \\
(x, y, x(z w))+(x, y,(w z) x) & =-(x, z w, y) x-x(x, w z, y)
\end{aligned}
$$

Então:

$\sum_{(y, z, w)}=x((x, z, y w)+(x, w, z y)+(x, y, w z))+((x, z, w y)+(x, w, y z)+(x, y, z w)) x$

Agora o Lema 4.6.10 (iv) nos dá:

$$
\begin{aligned}
\sum_{(y, z, w)} & =x[x,(z, y, w)]+[x,(y, z, w)] x=x[x,(z, y, w)]-[x,(z, y, w)] x \\
& =[x,[x,(z, y, w)]]=-[x,[x,(y, z, w)]]
\end{aligned}
$$

Portanto $f=[x,[x,(y, z, w)]]-[x,[x,(y, z, w)]]=0$

\section{Identidades de grau $[1,1,1,1,1]$}

Seja $f$ uma identidade de grau $[1,1,1,1,1]$, ou multilinear. Temos que $f$ é uma combinação linear de produtos de 5 elementos $x, y, z, v, w$ nas possíveis distribuições 
de parêntesis entre eles. São ao todo 14 distribuições, que constituirão nos 14 elementos geradores do ideal das identidades multilineares de grau $[1,1,1,1,1]$. Designaremos estes geradores por $d_{1}, \ldots, d_{14}$, explicitados a seguir:

$$
\begin{aligned}
& d_{1}=\_\left(\_\left(\_\left(\_\right)\right)\right) ; \quad d_{2}=\_\left(\_\left(\left(\_\_\right) \_\right)\right) ; \quad d_{3}=\_\left(\left(\_\left(\_\_\right)\right)\right) ; \\
& d_{4}=\_\left(\left(\left(\_-\right)_{-}\right)_{-}\right) ; \quad d_{5}=\_\left(\left(\_\_\right)\left(\_\_\right)\right) ; \quad d_{6}=\left(\_\left(\_\left(\_\_\right)\right){ }_{-} ;\right. \\
& d_{7}=\left({ }_{-}\left(\left(\_-\right)_{-}\right)\right)_{-} ; \quad d_{8}=\left(\left(\_\left(\_\_\right)\right)_{-}\right)_{-} ; \quad d_{9}=\left(\left(\left(\_\_\right)_{-}\right)_{-}\right)_{-} ;
\end{aligned}
$$

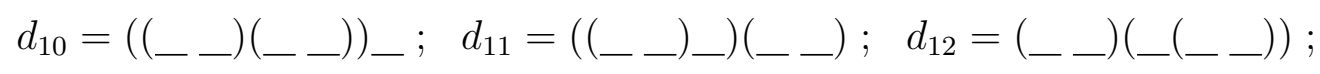

$$
\begin{aligned}
& d_{13}=\left(\_\_\right)\left(\left(\_\_\right) \_\right) ; \quad d_{14}=\left(\_\left(\_\_\right)\right)\left(\_\_\right)
\end{aligned}
$$

Faremos as seguintes substituições:
1. $e_{1}, e_{2}, e_{4}, e_{3}, e_{6}$
2. $e_{1}, e_{2}, e_{4}, e_{4},-e_{7}$
3. $e_{1}, e_{2}, e_{4}, e_{3}, e_{5}$
4. $e_{1}, e_{2}, e_{4}, e_{6},-e_{7}$
5. $e_{1}, e_{2}, e_{4}, e_{6}, e_{5}$
6. $e_{1}, e_{2}, e_{4},-e_{7}, e_{5}$
7. $e_{1}, e_{2}, e_{3}, e_{6},-e_{7}$
8. $e_{1}, e_{2}, e_{3}, e_{6}, e_{5}$
9. $e_{1}, e_{2}, e_{3},-e_{7}, e_{5}$
10. $e_{1}, e_{2}, e_{6},-e_{7}, e_{5}$
11. $1, e_{2}, e_{4}, e_{3}, e_{6}$
12. $1, e_{1}, e_{4}, e_{3}, e_{6}$
13. $1, e_{1}, e_{2}, e_{3}, e_{6}$
14. $1, e_{1}, e_{2}, e_{4}, e_{6}$
15. $1, e_{1}, e_{2}, e_{4}, e_{3}$

Após os cálculos obtemos a matriz dos coeficientes do sistema homogêneo nas incógnitas $\alpha_{1}, \ldots, \alpha_{14}$ indicada a seguir:

$$
\left(\begin{array}{rrrrrrrrrrrrrr}
1 & -1 & 1 & -1 & -1 & -1 & 1 & -1 & 1 & 1 & -1 & 1 & -1 & 1 \\
-1 & -1 & -1 & 1 & -1 & 1 & -1 & 1 & -1 & -1 & -1 & -1 & -1 & 1 \\
1 & -1 & -1 & 1 & 1 & -1 & 1 & -1 & 1 & 1 & -1 & 1 & -1 & 1 \\
-1 & 1 & 1 & 1 & 1 & 1 & 1 & 1 & -1 & 1 & -1 & 1 & -1 & 1 \\
-1 & 1 & 1 & 1 & 1 & 1 & 1 & 1 & -1 & 1 & 1 & 1 & -1 & -1 \\
1 & -1 & 1 & -1 & 1 & 1 & -1 & -1 & 1 & 1 & 1 & -1 & 1 & -1 \\
-1 & 1 & 1 & -1 & -1 & 1 & -1 & -1 & -1 & -1 & -1 & -1 & 1 & -1 \\
-1 & -1 & -1 & 1 & -1 & 1 & -1 & -1 & -1 & -1 & -1 & -1 & -1 & -1 \\
-1 & 1 & -1 & 1 & -1 & 1 & -1 & -1 & -1 & -1 & -1 & -1 & 1 & -1 \\
-1 & 1 & -1 & 1 & -1 & 1 & -1 & 1 & -1 & 1 & -1 & 1 & -1 & 1 \\
-1 & 1 & -1 & -1 & 1 & -1 & 1 & 1 & 1 & -1 & 1 & -1 & 1 & 1 \\
-1 & 1 & 1 & -1 & -1 & 1 & -1 & -1 & -1 & 1 & -1 & -1 & 1 & -1 \\
1 & -1 & -1 & -1 & -1 & -1 & -1 & -1 & -1 & -1 & -1 & 1 & -1 & -1 \\
-1 & -1 & -1 & 1 & -1 & -1 & 1 & 1 & 1 & -1 & -1 & -1 & -1 & -1 \\
-1 & 1 & -1 & 1 & 1 & -1 & 1 & 1 & 1 & -1 & 1 & -1 & 1 & 1
\end{array}\right)
$$


Após escalonamento obtemos a matriz:

$$
\left(\begin{array}{rrrrrrrrrrrrrr}
1 & 0 & 0 & 0 & 0 & 0 & 0 & 0 & 0 & 0 & 0 & 0 & 1 & 0 \\
0 & 1 & 0 & 0 & & & & & & & & 0 & 1 & 0 \\
& 0 & 1 & 0 & & & & & & & & & 0 & 0 \\
& & 0 & 1 & 0 & & & & & & & & & \\
& & & 0 & 1 & 0 & & & & & & & & 0 \\
& & & & 0 & 1 & 0 & & & & & & & 0 \\
& & & & & 0 & 1 & 0 & & & & & & 0 \\
& & & & & & 0 & 1 & 0 & & & & & 0 \\
& & & & & & & 0 & 1 & 0 & & & & 0 \\
& & & & & & & & 0 & 1 & 0 & & & 0 \\
& & & & & & & & & 0 & 1 & 0 & 0 & 0 \\
& & & & & & & & & 0 & 0 \\
& & & & & & & & & & 0 & 1 & -1 & 0 \\
& & & & & & & & & & 0 & 0 & 0 & 1 \\
& & & & & & & & & & 0 & 0 & 0 & 0 \\
0 & 0 & 0 & 0 & 0 & 0 & 0 & 0 & 0 & 0 & 0 & 0 & 0 & 0
\end{array}\right)
$$

Resultam as seguintes relações entre as variáveis, onde fizemos a variável livre $\alpha_{13}=\alpha:$

$$
\alpha_{1}=\alpha_{2}=-\alpha ; \quad \alpha_{12}=\alpha ; \quad \alpha_{3}=\alpha_{4}=\ldots=\alpha_{11}=\alpha_{14}=0
$$

A identidade $f$ poderá ser escrita como um múltiplo linear dos produtos designados por $d_{1}, d_{2}, d_{12}$ e $d_{13}$, uma vez que os coeficientes dos outros produtos se anulam nas substituições feitas. Temos portanto:

$$
\begin{aligned}
& f=\alpha \sum_{(x, y, z, v, w)}(-x(y(z,(v w)))-x(y((z v) w))+(x y)(z(v w))+(x y)((z v) w)), \\
& \text { onde } \sum_{(x, y, z, v, w)} \text { indica a soma sobre todas as permutações de } x, y, z, v, w .
\end{aligned}
$$

Podemos escrever esta soma como:

$$
\left(d_{13}-d_{1}\right)+\left(d_{12}-d_{2}\right)=(x y)((z v) w)-x(y(z,(v w)))+(x y)(z(v w))-x(y((z v) w))
$$

Note que:

$$
\begin{aligned}
(x, y,(z, v, w)) & =(x, y,(z v) w)-(x, y, z(v w)) \\
& =\underbrace{(x y)((z v) w)}_{d_{13}}-\underbrace{x(y((z v) w))}_{d_{2}}-\underbrace{x(y(z(v w)))}_{d_{1}}+\underbrace{(x y)(z(v w))}_{d_{12}}
\end{aligned}
$$

Portanto: $f=\alpha \sum_{(x, y, z, v, w)}(x, y,(z, v, w))$ 
Afirmação: $\sum_{(x, y, z, v, w)}(x, y,(z, v, w))=0$.

Vamos desenvolver esta soma sem precisar explicitar todos os $5 !=120$ termos resultantes da permutação de 5 elementos. Fixando duas variáveis, permutamos as outras três dentro do associador interno. Obtemos assim uma soma de 6 associadores para cada duas variáveis fixadas. Como temos $A_{5,2}=20$ maneiras de fixar duas dentre as cinco variáveis, teremos ao todo $6 \times 20=120$ termos, como deve ser.

As 20 somas podem ser representadas sinteticamente por:

$$
\begin{aligned}
\sum_{i=1}^{20} S_{i} & =[(x, y,(z, v, w))+\cdots+(x, y,(w, v, z))] \\
& +[(y, x,(z, v, w))+\cdots+(y, x,(w, v, z))] \\
& +[(x, z,(y, v, w))+\cdots+(x, z,(v, w, y))] \\
& +[(z, x,(y, v, w))+\cdots+(z, x,(w, v, y))] \\
& +\cdots+[(w, v,(x, y, z))+\cdots+(w, v,(z, y, x))]
\end{aligned}
$$

Basta provar que uma dessas somas é zero. Por simetria todas serão. Assim, explicitemos a primeira delas:

$$
\begin{aligned}
S_{1} & =[(x, y,(z, v, w))+\cdots+(x, y,(w, v, z))] \\
& =(x, y,(z, v, w))+(x, y,(z, w, v))+(x, y,(v, z, w))+(x, y,(v, w, z)) \\
& +(x, y,(w, q, v))+(x, y,(w, v, z)) \\
& =(x, y,(z, v, w))+(x, y,-(z, v, w))+(x, y,-(z, v, w))+(x, y,(z, v, w)) \\
& +(x, y,(z, v, w))+(x, y,-(z, v, w))=0
\end{aligned}
$$

Como todas as somas se anulam, a afirmação está provada. Portanto $f=0$.

Provamos assim que uma identidade de grau $[1,1,1,1,1]$ multilinear é nula. Portanto toda identidade de grau 5 não nula é conseqüência das identidades $\chi$ e $\kappa$, conforme afirmamos no início desta seção.

\subsection{Resultados Recentes}

Além das identidades de grau 5 que aqui apresentamos, existem também nas álgebras alternativas, identidades de grau 6 ou maior. Pesquisas recentes já explicitaram tais identidades ou, pelo menos, estabeleceram métodos mais gerais para sua deter- 
minação. Em particular, Hentzel e Peresi, em seu trabalho "Identities for CayleyDickson Algebras" (ref. [10]) utilizaram métodos computacionais. Destacamos também o artigo "Identities for the Associator in Alternative Algebras", de BremnerHentzel (ref. [2]) envolvendo álgebras ternárias alternativas, em que foram encontradas identidades de grau 7, também com o uso de computadores.

Daremos aqui uma breve noção destas pesquisas, procurando apresentar alguns exemplos das identidades estudadas, e dos métodos utilizados pelos autores.

Em [10] é utilizado um algoritmo computacional que permite gerar todos os polinômios multilineares homogêneos candidatos a identidades. Expressando tais polinômios em bases convenientes de matrizes, e como combinações lineares de elementos do anel de grupo $F S_{n}$, impõe condições para que sejam identidades. Deduzem assim, as expressões das identidades de grau $\leq 6$.

Trata-se de um processo indutivo de geração de identidades. Embora os autores tenham se limitado a apresentar indentidades de grau $\leq 6$, o processo permite, em teoria, encontrar identidades de qualquer grau. Porém, existem limitações de ordem prática. Por ser geral, produz também identidades que são conseqüências das leis alternativas, devendo ser, então, descartadas. Para identificar quais identidades são efetivamente novas, utiliza as formas canônicas de matrizes que podem ser associadas às identidades. Antes de fazer esta associação é preciso encontrar uma base para expressar um polinômio multilinear homogêneo (candidato a identidade). Mas a álgebra alternativa livre não tem uma base natural. Assim é utilizado um isomorfismo para o anel de grupo das matrizes completas sobre $F$. Utilizando alguns resultados destas matrizes, mostra que um polinômio pode ser associado a uma soma direta das mesmas, para a qual existe uma base conveniente (em termos computacionais), com elementos não nulos somente na primeira coluna.

Obtém assim candidatos a identidades, substituindo os elementos da base de $C(-1,-1,-1)$ e impondo uma lista de restrições sobre os coeficientes desses candidatos para que o polinômio seja uma identidade. Indicamos a seguir a identidade de grau 6 encontrada por este processo, onde $a, b, c, d, e, f$ representam elementos arbitrários em $C$ :

$$
\left[\sum\{24 a(b(c(d e)))+8 a((b, c, d) e)-11(a, b,(c, d, e))\}, f\right]=0
$$


Em [2], são pesquisadas identidades nas chamadas álgebras ternárias alternativas. Podemos definir, de maneira bem simples, uma álgebra ternária como sendo um par $(A, t)$, onde $A$ é um espaço vetorial sobre um corpo $F$ e $T: A \times A \times A \rightarrow A$ é uma função trilinear. Esta função serve para definir um produto em $(A, t)$, como $t(a, b, c)=(a b) c-a(b c)$, que nada mais é que o nosso conhecido associador. Com isto, $(A, t)$ se torna uma álgebra ternária.

Os autores apresentam identidades de grau 7 em três tipos de álgebras ternárias:

1. Álgebras Alternativas Livres;

2. Álgebras de Cayley;

3. Produto Cruzado.

Indicaremos aqui somente as identidades referentes ás álgebras ternárias de Cayley. Ressaltam os autores que os cálculos foram feitos em computador com um corpo de 103 elementos (característica 103), mas como o grau das identidades é $\leq 7$, que é muito menor que 103, é provável que esta restrição seja desnecessária. Assim, eles acreditam que os resultados valem em característica zero, o que foi verificado em alguns casos particulares. Mas a questão permanece em aberto.

Da mesma forma que no artigo anterior, aqui é usada a representação de identidades polinomiais por elementos do anel de grupo $F S_{n}$, que se apóia na teoria de representações. Conseguem assim uma forma conveniente de representação computacional para um polinômio, em termos de soma direta de matrizes. São apresentadas cinco identidades de grau 7, que são chamadas de não-óbvias. Mais especificamente, eles assinalam tratar-se de identidades satisfeitas pela associador, uma vez que o produto nestas álgebras é dado pelo associador. Antes de apresentar estas cinco identidades, vamos esclarecer alguns pontos quanto à notação utilizada pelos autores.

Para o associador, utilizam a notação $(a b c)$ ao invés de $(a, b, c)$, destacando o seu papel de produto nesta álgebra. Em um polinômio multilinear homogêneo $I$ em que uma letra, digamos $x$, ocorre pelo menos duas vezes, é definida a soma alternada sobre as posições de $x$, denotada por $\sum_{\text {alt }(x)} I$. De forma sucinta, este operador toma a soma alternada sobre $k$ ! permutações sobre uma lista de $k$ letras (que não ocorrem em $I$ ), onde $k$ indica o número de ocorrências da letra $x$ em cada termo de $I$. Tal processo é chamado de linearização parcial alternada. Na verdade, $x$ faz o papel de 
indeterminada, e os autores alertam para não se confundir entre ( $a x x)$, que é zero em uma álgebra alternativa, e $\sum_{a l t(x)}(a x x)$, onde o $x$ deve ser substituído por outras letras, digamos $b$ e $c$, para se fazer a expansão em somas alternadas antes de se calcular o produto ternário. As cinco identidades de grau 7 satisfeitas pelo associador em uma álgebra de Cayley são as seguintes:

$$
\begin{aligned}
C_{1}= & \sum_{a l t(x)}\{(((a b x)(a x x) b)-((a b x)(b x x) z)+2((a b x)(a b x) x) \\
& +2(((a b x) a x) b x)-2(((a b x) b x) a x)-(((a x x) b x) a b) \\
& +(((b x x) a x) a b)-2(((a b x) x x) a b)+3(((a b x) a b) x x) \\
& +(((x x x) a b) a b)+4(((a x x) a b) b x)-4(((b x x) a b) a x)\} ;
\end{aligned}
$$

Ao encerrarmos este trabalho, chamamos a atenção para alguns aspectos da pesquisa de identidades em álgebras alternativas que nos pareceram relevantes. Em primeiro lugar, o caráter nada elementar das expressões obtidas, que apresentam uma complexidade crescente a cada aumento de grau. Daí o seu tratamento exigir a utilização de diversos recursos como, triplo produto de Jordan, derivação, linearização, bases do ideal associador, grupos de permutação, etc. Vemos então que a abrangência do tema, por si só, já representa uma motivação para o seu estudo.

Salientamos também a utilização de técnicas computacionais na avaliação das identidades, o que se faz necessário devido ao grande número de operações envolvido. Este fato ressalta a importância do computador como ferramenta auxiliar neste tipo de pesquisa matemática.

Por fim, deixamos como sugestão para futuras pesquisas, a investigação de novas identidades, uma vez que algumas questões permanecem em aberto, e ainda, os novos resultados poderão trazer significativas contribuições para a Matemática. 


\section{Bibliografia}

[1] Albert, A.A. Structures of Algebras. American Math Soc., New York, 1939.

[2] Bremner, M. and Hentzel, I.R. Identities for the associator in alternative algebras. J. Symbolic Computation, 33:255-273, 2002.

[3] Bruck, R.H. and Kleinfeld, E. The structure of alternative division rings. Proc. American Math., (2):878-890, 1951.

[4] Drenski, V.S. A minimal basis of identities for a second-order matrix algebra over a field of characteristic 0. Algebra i Logika, 20:188-194, 1982.

[5] Farenick, D.R. Algebras of Linear Transformation. Springer-Verlag, New York, 2001.

[6] Guzzo, Jr. H. O Teorema de Frobenius para álgebras não associativas. IMEUSP, 1985. Dissertação de mestrado.

[7] Guzzo, Jr. H. The bar-radical of baric algebras. Arch. Math., 67(2):106-118, 1996.

[8] Guzzo, Jr. H. and Couto, M.A. The radical in alternative baric algebras. Arch. Math. (Basel), 75(3):178-187, 2000.

[9] Hentzel, I.R. Processing identities by group representation. In Computers in Non-associative Rings and Algebras, pages 13-40. R.E. Beck and B. Kolmann, eds., Academic Press, New York, 1977.

[10] Hentzel, I.R and Peresi, L.A. Identities of Cayley-Dickson algebras. $J$. Algebra, 188:292-309, 1997. 
[11] Herstein, I.N. Noncommutative Rings. The Mathematical Association of America, USA, 1973.

[12] Herstein, I.N. Rings with Involution. University of Chicago Press, Chicago, 1976.

[13] Jacobson, N. Basic Algebra I. Van Nostrand, New York, 1974.

[14] Kuramochi, C. C. Identidades Polinomiais para as Álgebras de Matrizes, Álgebras de Jordan de grau 2 e Álgebras de Cayley-Dickson. IME-USP, 1994. Dissertação de mestrado.

[15] Kuzmin, E.N. and Shestakov, I.P. Non-associative structure. In Algebra VI. Encyclopedia of Mathematical Sciences, volume 57. Kostrikin, A.I. Shafarevich, I.R. eds., Springer-Verlag, New York, 1995.

[16] Milies, C.P. Breve história da Álgebra abstrata. II Bienal da SBM, UFBA, 2004.

[17] Procesi, C. Polynomial Identities in Algebras. Marcel Dekker, New York, 1973.

[18] Racine, M.L. Minimal identities of Jordan algebras of degree 2. Communications in Algebra, 13:2493-2506, 1985.

[19] Racine, M.L. Minimal identities of octonion algebras. Journal of Algebra, 115:251-260, 1988.

[20] Rosset, S. A new proof of the Amitsur-Levitzki identity. Israel Journal of Mathematics, 23(2):187-188, 1976.

[21] Rowen, L.H. Polynomial Identities in Ring Theory. Academic Press, New York, 1980.

[22] Schafer, R.D. An Introduction to Nonassociative Algebras. Dover Publications, New York, 1995.

[23] Shephard, G.C. Vector Spaces of Finite Dimension. John Wiley \& Sons, New York, 1966. 
[24] Wörz-Busekros, A. Algebras in Genetics, volume 36 of Lecture Notes in Biomathematics. Springer-Verlag, Berlin/New York, 1980.

[25] Zhevlakov, K.A., Slin'Ko, A.M., Shestakov, I.P., and Shirshov, A.I. Rings that are nearly associative. Academic Press, New York, 1982. 